
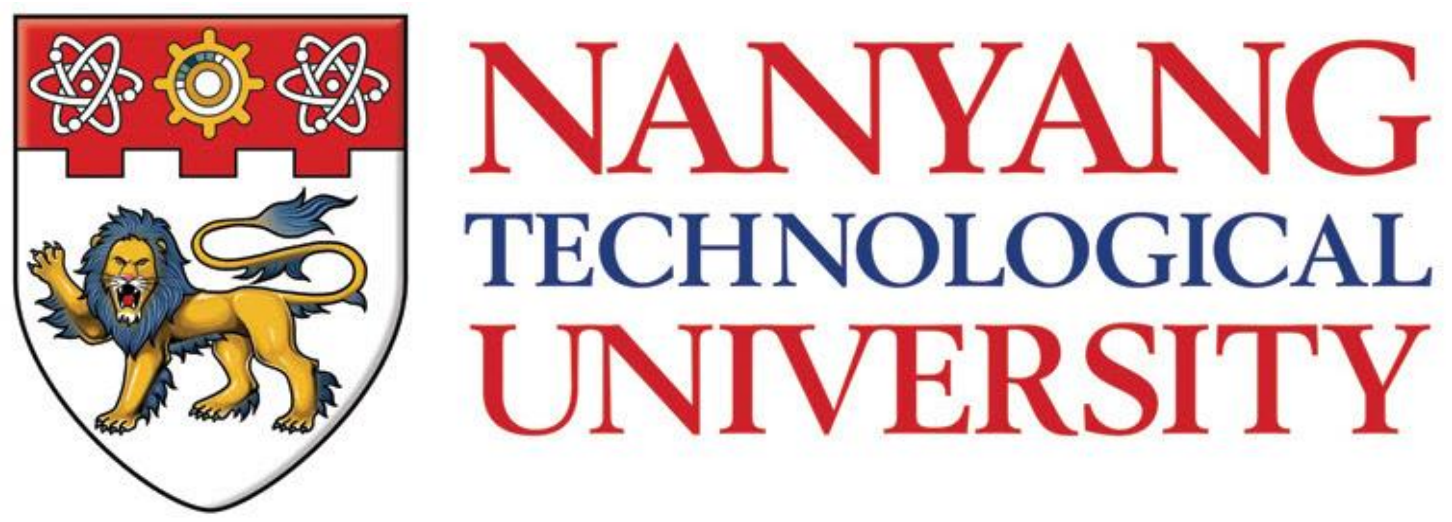

MOLECULAR MECHANISMS UNDERLYING SELECTIVE CLEARANCE OF PROTEIN AGGREGATES AND MITOCHONDRIA BY AUTOPHAGY

TAN SIJIE SCHOOL OF BIOLOGICAL SCIENCES 2017 


\section{MOLECULAR MECHANISMS UNDERLYING SELECTIVE CLEARANCE OF PROTEIN AGGREGATES AND MITOCHONDRIA BY AUTOPHAGY}

TAN SIJIE

\section{SCHOOL OF BIOLOGICAL SCIENCES}

A thesis submitted to the Nanyang Technological University in partial fulfilment of the requirement for the degree of Doctor of Philosophy 


\section{ACKNOWLEDGEMENTS}

First and foremost, I will like to express my sincere gratitude to my supervisor Prof. Esther Wong for the continuous support for my Ph.D. study, and the sharing of her immense knowledge and insightful comments that have helped me through the 4.5 years of candidature. She has opened up a lot of opportunities for me to engage with the international scientific committee, by giving me several chances to participate and present in international and local conferences, as well as writing review papers. In addition, she has given me multiple opportunities to take up different projects, which led me to be able to provide this thesis with substantial amount of research data which I am honored with. Looking back, it still amazes me on how much I can complete and achieve during my candidature period. But all these wouldn't be made possible without her help and guidance. Working with my supervisor has made me grow as a person and realized the potential within me. Although there were some ups and downs in the journey, but upon reflecting, the Ph.D. experience and memories are valuable and positive.

Besides my supervisor, I would like to thank my Thesis Advisory Committee, examiners for my Qualifying Exam, and my Ph.D. thesis committee: Prof. Valerie Lin, Prof. Oliver Martin Mueller-Cajar, Prof. Thirumaran s/o Thanabalu, Prof. Liou Yih Cherng and Prof. Ben Loos for their insightful comments.

I will also like to thank the students and colleagues I have worked with: Project officers Sim Zhi Wei, Ng Chye Yun, Lau Hwee Hui and Karen Lee for taking care of the lab and making the whole working experience a very pleasant one; Eureka/FYP students Brianna Lee, Jun Xiang, Jasmine Png and Faith See for their contributions towards the functional food project; A special thank goes to my fellow Ph.D. colleague/mate Moumita Rakshit for all the help and advice given. Last but not least, I would like to thank my family for seeing me through this 4.5 years, and especially at the final 2 weeks in lieu of thesis submission. My Ph.D. journey will definitely not be completed without them. 
INDEX

\section{TABLE OF CONTENTS}

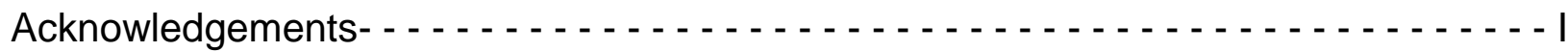

Table of contents $\ldots \ldots \ldots \ldots \ldots \ldots \ldots \ldots \ldots \ldots$

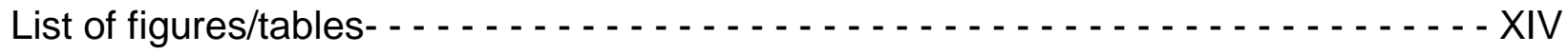

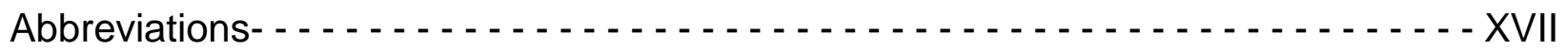

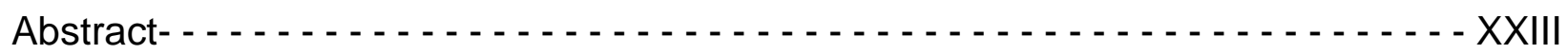


INDEX

\section{CHAPTER 1: INTRODUCTION}

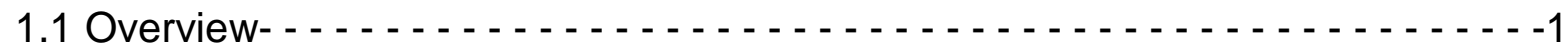

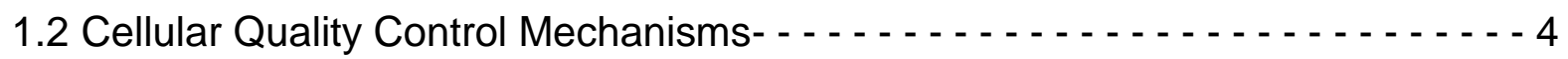

1.2.1 Molecular chaperones in protein folding and proteostasis

1.2.2 Ubiquitin-proteasome system (UPS)

1.2.3 Autophagy

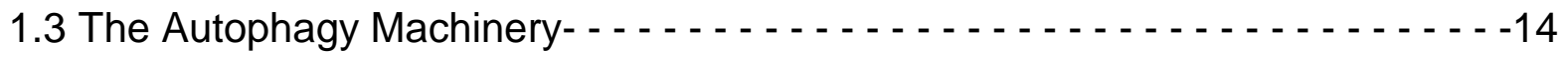

1.3.1 The core autophagy machinery

1.3.2 Regulators of autophagy induction

1.4 The Autophagy Hierarchy and Selective Autophagy - . . . . . - . . - . - 22

1.4.1 Basal and inducible autophagy

1.4.2 Selective autophagy

1.5 Proteostasis and Aggrephagy- - 25

1.5.1 Protein folding and misfolding

1.5.2 Protein aggregation and formation of inclusion bodies

1.5.3 Compartmentalization as a cytoprotective mechanism against proteotoxicity

1.5.4 The aggrephagy cascade

1.5.5 Regulation of the aggrephagy cascade

1.5.6 Non-canonical aggrephagy

1.6 Synphilin-1: An a-synucleopathies-associated Protein Implicated in Modulating Cargo Adaptor-Independent Aggrephagy- - - . - . - . - . - . - . - - - 31

1.6.1 Synphilin-1 is robustly ubiquitinated and phosphorylated to regulate aggregation behavior

1.6.2 Synphilin-1 harbors Agm-targeting motif 
1.6.3 Synphilin-1 ANK1 domain regulates autophagic susceptibility of Agg and Agm by basal and inducible aggrephagy

1.7 Aim 1: Understanding the Mechanism underlying Synphilin-1 Mediated

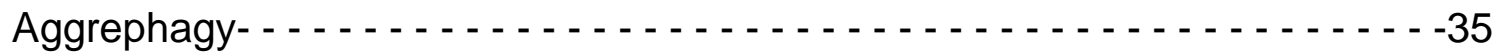

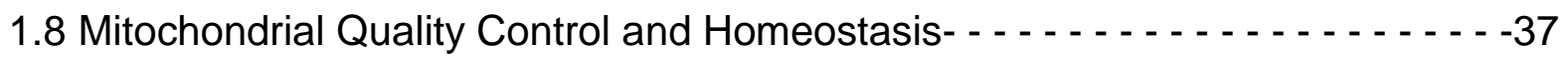

1.8.1 Mitochondrial biogenesis

1.8.2 Mitochondrial quality control mechanisms

1.8.3 Mitophagy regulation by mitochondrial dynamics

1.8.4 Canonical mitophagy: PINK1-Parkin mediated mitophagy

1.8.5 OMM proteins-mediated mitophagy

1.8.6 Lipid-mediated mitophagy

1.8.7 Coordination between mitochondrial biogenesis and mitophagy maintains mitochondrial homeostasis

1.9 Impairments in Aggrephagy and Mitophagy in Neurodegeneration- - - - - - - 48

\subsubsection{Parkinson's disease}

1.9.2 Huntington's disease

1.9.3 Amyotrophic lateral sclerosis

1.10 Pharmacological Interventions to Modulate Autophagy as a Therapy for

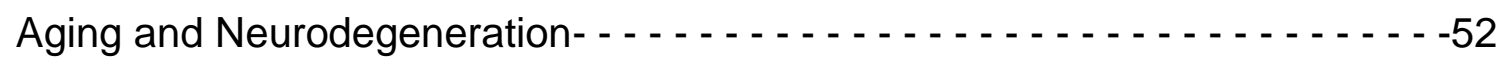

1.10.1 Enhancing systemic autophagy activity: Targeting mTOR and AMPK signaling pathways

1.10.2 Enhancing selective autophagy: Aggrephagy and mitophagy

1.11 Regulating Autophagy via Dietary Modulation- - - . - - - - 56

1.11.1 Caloric restriction

1.11.2 Polyphenols and the protection against protein aggregates-induced neurotoxicity 
1.12 Aim 2: To Investigate the Potential of Pomegranate as a Functional Food

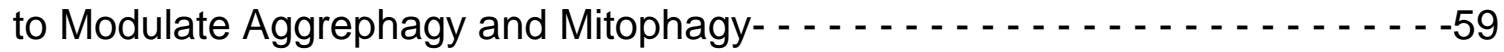


INDEX

\section{CHAPTER 2: MATERIALS AND METHODS}

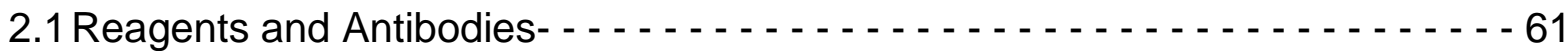

2.1.1 Plasmids

2.1.2 Primary antibodies

2.1.3 Secondary antibodies

2.1.4 Inhibitors

2.1.5 Mitochondrial depolarization and protein oxidation

2.1.6 Pomegranate extract (PE)

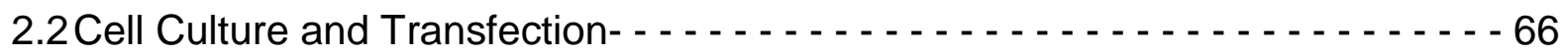

2.2.1 Growth conditions

2.2.2 Transfection and treatments

2.2.3 Generation of mCherry-Parkin, GFP-TFEB and MitoTimer stable cells

2.2.4 Short interfering RNA (siRNA)-mediated knockdown (KD) in SY5Y cells

2.3RNA Extraction and Reverse Transcription-PCR (RT-PCR) - - - - - - - - - - -69

2.4Cell Lysis, Sodium Dodecyl Sulfate Polyacrylamide Gel Electrophoresis (SDS-

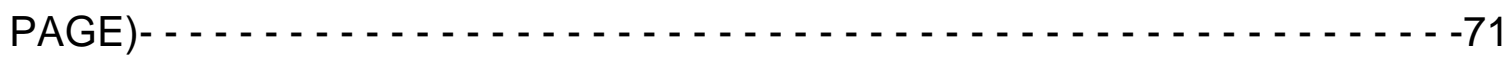

2.4.1 Cell lysis

2.4.2 SDS-PAGE

2.4.3 Densitometry analysis

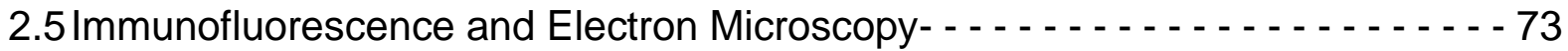

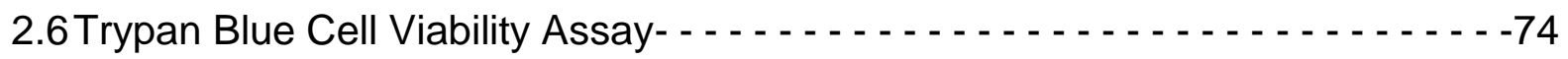

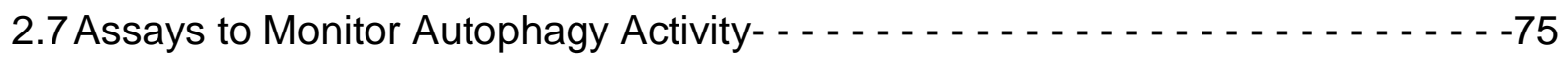

2.7.1 LC3-II flux

2.7.2 GFP-mRFP-LC3 autophagic flux 
2.7.3 Immunofluorescence analysis of the number and size of autophagosomes and lysosomes

2.8TFEB Nuclear Localization-

2.9 Assays to Investigate Phosphorylation Status of Synphiin-1 - - _ - - - - - - 79

2.9.1 Mass spectrometry

2.9.2 in-silico analysis

2.9.3 PhosTag gel

2.10 Assays to Investigate Synphilin-1 Aggrephagy Mechanisms- - - - - - - - - 82

2.10.1 Formation and clearance of Sph1 inclusion bodies under kinase inhibitions

2.10.2 Analysis of the presence of various protein kinases in Sph1 inclusions via colocalization studies

2.10.3 Immunoprecipitation (IP) to evaluate ubiquitination on Sph1

2.10.4 Co-immunoprecipitation (co-IP) to investigate the interactions between Parkin and Sph1 with protein kinases

2.11 Oxyblot Assay to Monitor Protein Oxidation $\ldots$

2.12 Assays to Monitor Mitochondrial Health and Mitophagy- - - - - - - - - -86

2.12.1 Mitochondrial fractionation

2.12.2 Quantification of mitochondrial morphology

2.12.3 Quantification of LC3, PINK1 and Parkin colocalizations per mitochondria content

2.12.4 CCCP-induced mitochondria disappearance and accumulation under autophagy inhibition (mitochondrial flux)

2.12.5 MitoTimer and MitoSox

2.13 Statistical Analysis $\ldots \ldots$ 
INDEX

\section{CHAPTER 3: INTERPLAY BETWEEN PHOSPHORYALTION AND UBIQUITINATION REGULATES SYNPHILIN-1 MEDIATED AGGREPHAGY}

3.1 Background $\ldots$

3.2 Identification of Phosphorylated Sites in Synphilin-1 by in-silico Analysis and Mass

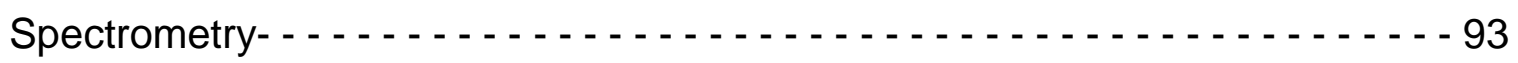

3.2.1 in-silico analysis

3.2.2 Mass spectrometry analysis of Sph1 phosphorylation

3.3 Synphilin-1 Associates with PKC and GSK3 $\beta$ Isoforms- - - - - - - - - - - - 100

3.3.1 Sph1 interacts with PKCa, PKCy and GSK3 $\beta$

3.3.2 PKCa, PKCy, CKII $\beta$ and GSK3 $\beta$ localized to Agg and Agm formed by Sph1

3.4 Synphilin-1 is Highly Phosphorylated under Proteasomal Stress- - - - - - - - - 108

3.5 Pharmacological and Genetic inhibition of PKC, CKII and GSK3 $\beta$ on Synphilin-1

Protein Expression, Aggregation and Aggrephagy Experiments- - - - - - - - - -112

3.5.1 Inhibitions of PKC, CKII and GSK3 $\beta$ did not affect Sph1 protein expression

3.5.2 PKC, CKII and GSK3 $\beta$ influence the levels of Agg and Agm formed by Sph1

3.5.3 Chemical inhibitions of PKC, CKII and GSK3 $\beta$ perturb clearance of Sph1 Agg by basal aggrephagy

3.5.4 Genetic ablation of PKC, CKII and GSK3 $\beta$ perturb clearance of Sph1 Agg by basal aggrephagy

3.5.5 Chemical inhibitions of PKC, CKII and GSK3 $\beta$ perturb clearance of Sph1 Agm by inducible aggrephagy

3.5.6 Genetic ablation of PKC, CKII and GSK3 $\beta$ perturb clearance of Sph1 Agm by inducible aggrephagy 
3.6 Modulation of Synphilin-1 Aggrephagy Behavior Under Kinase Inhibitions is not a Consequence to the Impairment of the General Autophagy Pathway - . - . - - 131

3.7GSK3 $\beta$ Modulates K63U on Synphilin-1 _ . . . . . . . . . . . . . . . . . 133

3.8GSK3 $\beta$ Interacts with Parkin and Modulates Synphilin-1 and Parkin Interaction- - - 136

3.8.1 Background: Parkin as an E3 ligase that modulates K63U on Sph1 and inducible aggrephagy

3.8.2 PKC $\alpha$ and GSK3 $\beta$ interact with Parkin under proteasomal stress

3.8.3 CKII and GSK3 $\beta$ regulate the interaction between Sph1 and Parkin under proteasomal stress

3.9 CKII and GSK3 $\beta$ Regulates Activation of Parkin by Phosphorylation- - - - - - - 142 
INDEX

\section{CHAPTER 4: POMEgRANATE ACTIVATES TFEB TO PROMOTE AUTOPHAGY-LYSOSOMAL FITNESS AND MITOPHAGY FOR MITOCHONDRIAL STRESS}

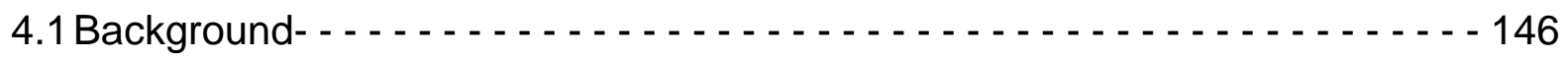

4.2 PE Upregulates Autophagy-Lysosomal Fitness- - - - - - - - - - - - - - - - - -

4.2.1 PE upregulates the level of autophagosomes in multiple cell type

4.2.2 PE upregulates lysosomal compartment

4.2.3 Electron micrography analysis of PE treated SY5Y cells

4.2.4 PE enhances autophagy flux

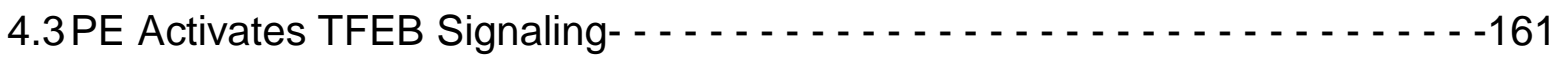

4.3.1 PE promotes TFEB nuclear localization and activation

4.3.2 PE activates TFEB via TFEBSer142 dephosphorylation

4.4 Lysosomal-Ca $^{2+}$ Signaling Pathway underlies PE-induced TFEB Activation- - - 167

4.4.1 PE-induced TFEB activation is not dependent on ERK2 signaling

4.4.2 PE-induced TFEB activation is not dependent on AKT-mTORC1 signaling

4.4.3 PE activates lysosomal- $\mathrm{Ca}^{2+}$ signaling pathway

4.5PE Protects against Oxidative Protein Damage and Protein Aggregation

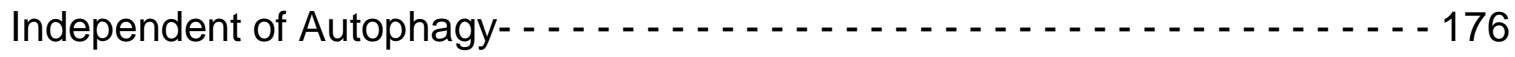

4.5.1 PE reduces $\mathrm{H}_{2} \mathrm{O}_{2}$-induced protein oxidation and enhances cell viability independent of autophagy pathway

4.5.2 PE alleviates aggregation-induced toxicity by reducing formation of protein aggregates 
4.6 PE promotes Autophagic Clearance of Damaged Mitochondria in Response to Mitochondrial Dysfunction and Mitigates Mitochondria Redox Toxicity- - - - - - 182

4.6.1 CCCP promotes TFEB activation via lysosomal- $\mathrm{Ca}^{2+}$ signaling pathway

4.6.2 PE alters mitochondria morphology

4.6.3 PE promotes recruitment of autophagosomes to the mitochondria basally and under CCCP-induced mitochondrial stress

4.6.4 PE promotes Parkin-PINK1 dependent mitophagy under CCCP-induced mitochondrial stress

4.6.5 PE promotes degradation of mitochondria under CCCP-induced stress

4.6.6 PE maintains mitochondrial homeostasis by preventing accumulation of damaged mitochondria and reduces mitochondrial ROS during mitochondrial stress

4.7 Major phytochemical components of PE regulate autophagy-lysosomal fitness and mitophagy-mediated stress resistance differently- - . - . - . - . - . - . - 2 202

4.7.1 PE bioactive components, punicalagin, delphinidin and luteolin, contribute to PE ability to upregulate autophagy via TFEB activation

4.7.2 Phenolic compound luteolin promotes mitophagy to protect against damaged mitochondria-induced redox toxicity

4.8TFEB knockdown abolishes PE protection against mitochondrial malfunction- 208

4.8.1 Ablation of TFEB function prevents PE mediated increase in autophagylysosomal compartments

4.8.2 Ablation of TFEB function reduces PE-mediated mitophagy and protection against mitochondrial $\mathrm{ROS}$ redox toxicity 
INDEX

\section{CHAPTER 5: DISCUSSIONS, FUTURE DIRECTIONS AND CONCLUSION}

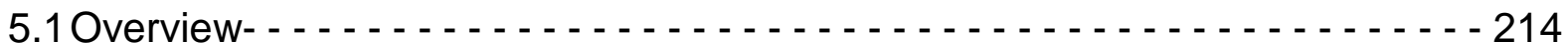

5.2 Interplay between Phosphorylation and Ubiquitination Modulates Synphilin-1

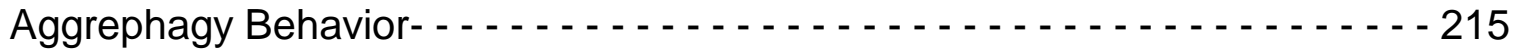

5.2.1 Phosphorylation represents a novel molecular determinant to regulate selective clearance of Agg by basal autophagy

5.2.2 Phosphorylation regulates selective clearance of Agm by inducible aggrephagy

5.2.3 GSK3 $\beta$ mediates the crosstalk between phosphorylation and K63U to regulate selective clearance of Agm by inducible aggrephagy

5.2.4 PKC and CKII modulates the selective clearance of Agm by inducible aggrephagy

5.2.5 Proteasomal stress potentiates the switch from basal to inducible aggrephagy

5.2.6 Sph1 in health and diseases: Sph1 as a novel adaptor protein to promote clearance of protein inclusions

5.3Pomegranate Promotes Mitophagy via TFEB Activation to Maintain Mitochondrial

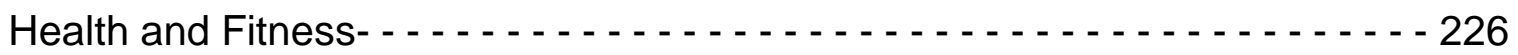

5.3.1 PE upregulates autophagy

5.3.2 PE activates TFEB via lysosomal- $\mathrm{Ca}^{2+}$ signaling independent of mTOR

5.3.3 PE-induced autophagy does to contribute to cellular proteostasis

5.3.4 PE modulates mitochondrial dynamics to prime the mitochondria for autophagic degradation

5.3.5 PE enhances PINK1-Parkin dependent mitophagy to mitigate mitochondrial redox toxicity under mitochondrial stress

5.3.6 Implications of PE in health and diseases 
INDEX

REFERENCE AND APPENDIX $\ldots$ 
INDEX

\section{LIST OF FIGURES}

\section{Chapter 1: Introduction}

- Fig. 1.1 The autophagy pathways

- Fig. 1.2 The autophagy machinery and the regulation by mTORC1, AMPK and TFEB signaling

- Fig. 1.3 The mitophagy pathways

Chapter 3: Interplay between Phosphorylation and Ubiquitination Regulates Synphilin-1-mediated Aggrephagy

- Fig. 3.1 Schematic diagram depicting K63U modulation of Sph1 aggrephagy behavior

- Fig. 3.2 Phosphorylation sites and kinases that modify Sph1 predicted by ScanProsite and Scansite 3.0 programmes

- Fig. 3.3 Mass spectrometry analysis detected multiple phosphorylation sites on Sph1

- Fig. 3.4 PKCy interacts with Sph1 basally and under proteasomal stress, but PKCa and GSK3 $\beta$ interact with Sph1 in response to proteasomal stress

- Fig. 3.5 PKCa, PKCy, CKII $\beta$ and GSK3 $\beta$ localized to the Agg and Agm formed by Sph1

- Fig. 3.6 Inhibitions of PKC, CKII and GSK3 $\beta$ differentially modulate phosphorylation of Sph1 under proteasomal stress

- Fig. 3.7 Inhibitions of PKC, CKII and GSK3 $\beta$ did not alter Sph1 protein expression

- Fig. 3.8 PKC, CKII and GSK3 $\beta$ influence the formation of Agg and Agm by Sph1

- Fig. 3.9 Inhibitions of PKC, CKII and GSK3 $\beta$ perturb clearance of Sph1 Agg by basal aggrephagy

- Fig. 3.10 siRNA-mediated KD of PKC $\alpha$, PKCY and GSK3 $\beta$ perturb clearance of Sph1 Agg by basal aggrephagy

- Fig. 3.11 PKC, CKII and GSK3 $\beta$ inhibitions perturb clearance of Sph1 Agm by starvation-induced aggrephagy 
- Fig. 3.12 siRNA-mediated KD of PKCa, PKCY and GSK3 $\beta$ perturb clearance of Sph1 Agm by starvation-induced aggrephagy

- Fig. 3.13 PKC, CKII and GSK3 $\beta$ inhibitions did not influence the cellular autophagic activity

- Fig. 3.14 Inhibition of GSK3 $\beta$ reduces K63U linkages on Sph1 under proteasomal stress

- Fig. 3.15 PKC $\alpha$ and GSK3 $\beta$ interact with Parkin in response to proteasomal stress

- Fig. 3.16 Inhibitions of CKII and GSK3 $\beta$ perturb the interaction between Parkin and Sph1 under proteasomal stress

- Fig. 3.17 Inhibition of CKII reduces the levels of P-Parkin ${ }^{565}$, while inhibition of GSK3 $\beta$ perturbs P-Parkin ${ }^{565}$ and cellular P-Ub ${ }^{565}$ levels

\section{Chapter 4: Pomegranate Activates TFEB to Promote Autophagy-Lysosomal Fitness and Mitophagy for Mitochondrial Stress}

- Fig. 4.1 PE upregulates the autophagosomal compartment in multiple cell types

- Fig. 4.2 PE upregulates lysosomal compartment in SY5Y cells

- Fig. 4.3 Electron microscopy analysis reveals high prevalence of autophagic vacuoles and lysosomes in PE treated SY5Y cells

- Fig. 4.4 PE increases autophagic flux

- Fig. 4.5 PE induces TFEB nuclear localization

- Fig. 4.6 PE inhibits TFEB ${ }^{\$ 142}$ phosphorylation in SY5Y cells

- Fig. 4.7 PE does not influence ERK1/2 activation and signaling

- Fig. 4.8 PE activates AKT and mTORC1 signaling pathways

- Fig. 4.9 PE activates TFEB via $\mathrm{Ca}^{2+}$-calcineurin signaling

- Fig. 4.10 PE protects against $\mathrm{H}_{2} \mathrm{O}_{2}$ oxidative stress-induced protein oxidation and cell death independent of autophagy pathway

- Fig. 4.11 PE inhibits protein aggregation of GFP-Sph1 under proteasomal stress

- Fig. 4.12 CCCP induces TFEB nuclear localization via lysosomal-Ca ${ }^{2+}$ calcineurin signaling 
- Fig. 4.13 PE alters mitochondrial morphology

Fig. 4.14 PE promotes recruitment of autophagosomes to the mitochondria under basal and CCCP-induced stress conditions

- Fig. 4.15 PE promotes recruitment of PINK1 and Parkin to mitochondria under CCCPinduced stress

- Fig. 4.16 PE promotes autophagic clearance of mitochondria (mitophagy) under CCCP-induced stress

- Fig. 4.17 PE reduces damaged mitochondria and alleviates mitochondrial redox toxicity

- Fig 4.18 Punicalagin, delphinidin chloride and luteolin upregulate autophagosomal and lysosomal compartments via TFEB activation

- Fig. 4.19 Luteolin promotes recruitment of autophagosomes to mitochondria under mitochondrial stress to facilitate mitochondria turnover and alleviate mitochondrial ROS toxicity

- Fig. 4.20 siRNA-mediated TFEB knockdown (KD) abolishes PE-induced upregulation of autophagosomal and lysosomal compartments

\section{LIST OF TABLES}

\section{Chapter 2: Materials and Methods}

- Table 1: siRNA sequences against TFEB, PKCa, PKCY, CKII $\beta$ and GSK3 $\beta$ 
INDEX

\section{ABBREVIATIONS}

$\underline{0-9}$

4BP1- - . - . - Eukaryotic Initiation Factor 4E Binding Protein

$\underline{A}$

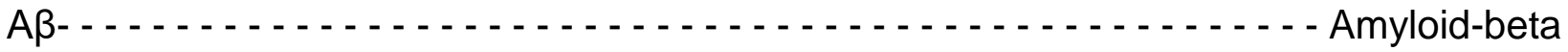

AD - .

Agm - .

AICAR - . . . 5-amino-4-carboxamide-1- $\beta$-D-ribonucleoside

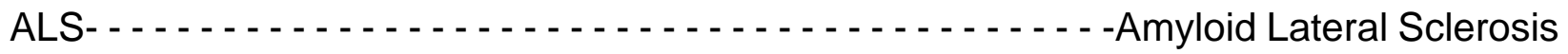

AMPK- .

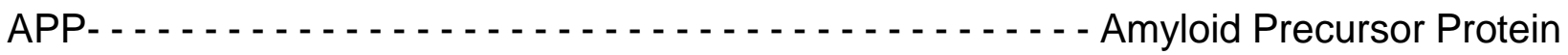

Atg -

ATP

$\underline{B}$

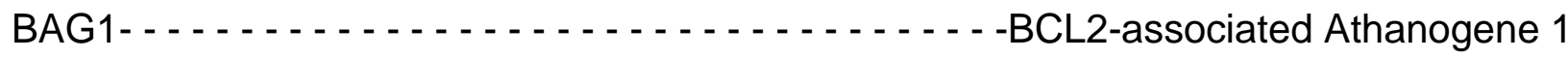

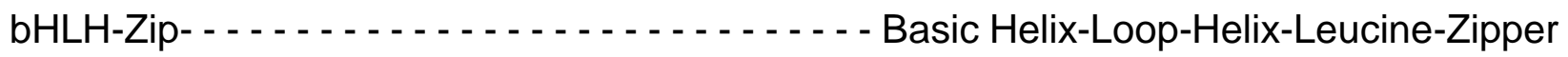
BNIP3 - . - . - . - . - . - BCL2/adenovirus E1B $19 \mathrm{kDa}$ protein-interacting protein 3

$\underline{\mathbf{C}}$

CCCP CCT $\ldots$

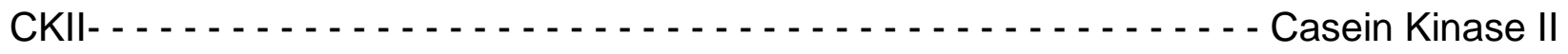




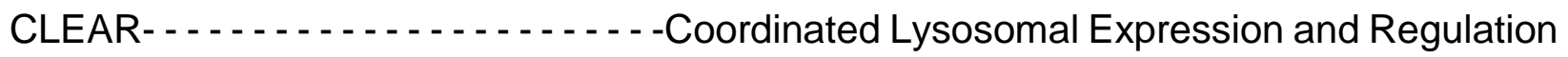
CMA

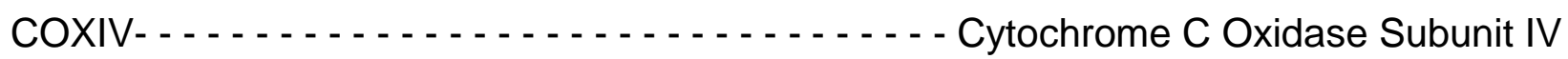

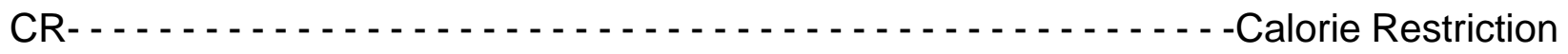

$\underline{\mathbf{D}}$

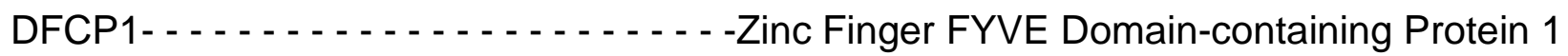

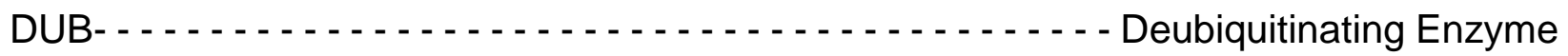

$\underline{E}$

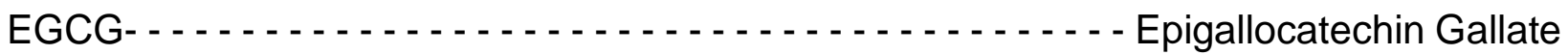
ERR - . . . . Estrogen-related Receptors

$\underline{F}$

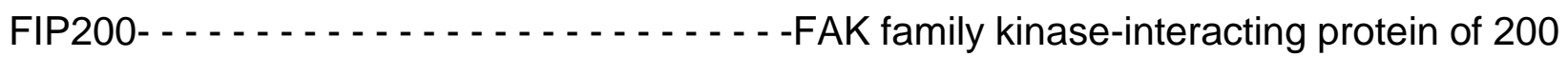
FOXO-

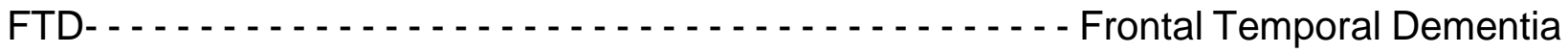

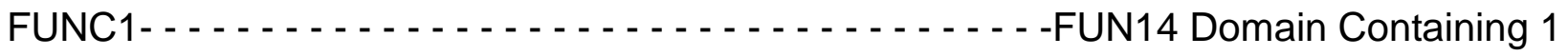

$\underline{\mathbf{G}}$

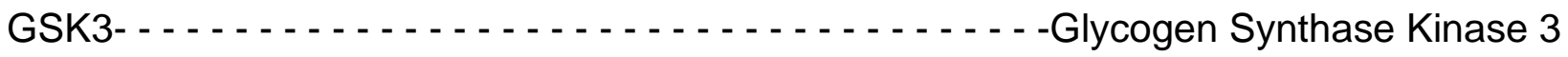
GSS- . . . . . . . . . . . . . . . . . . . . . . . . . . .

$\underline{\mathbf{H}}$

HD $\ldots \ldots$ Huntington's Disease 
HDAC6 - $\ldots$ -

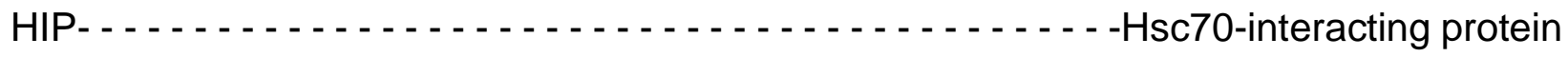

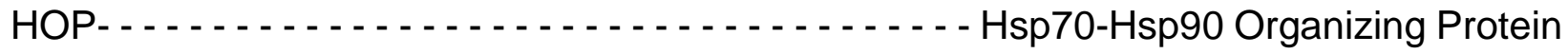
HOPS - . - Homotypic Fusion and Protein Sorting Complex

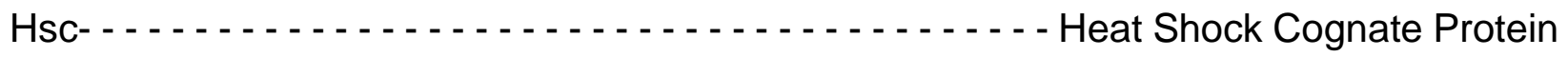
HSP . . . . . . . . . . . . . . . . . . . . . . . . . . . . . . . Htt - _ .

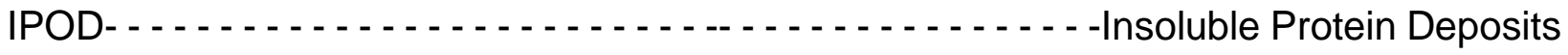

$\underline{\mathbf{J}}$

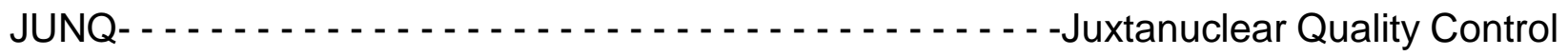

$\underline{\underline{L}}$

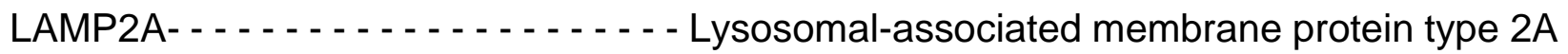

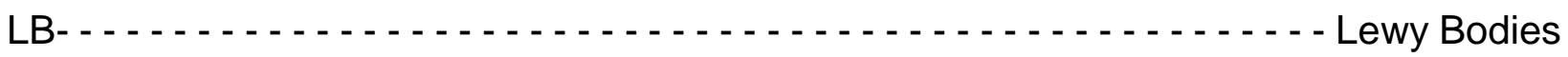
LC3-II- - . - . - . . - Microtubule-associated Protein 1A/1B-Light Chain 3 form II

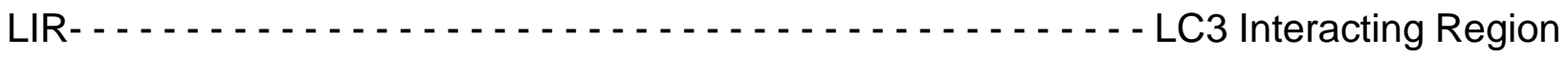

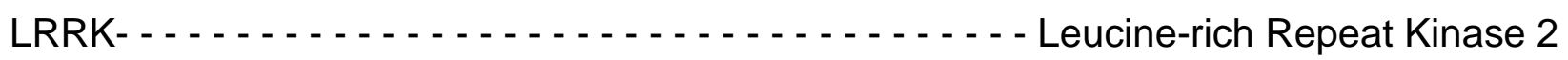

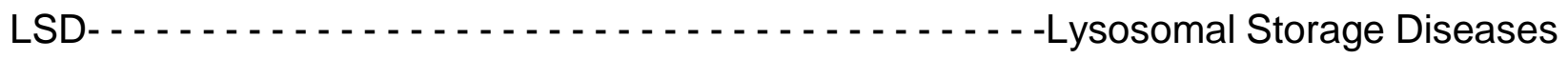

\section{$\underline{M}$}

MPP MPTP 
$\underline{\mathbf{N}}$

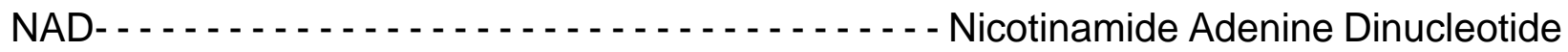

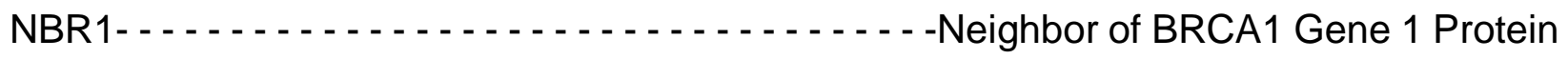

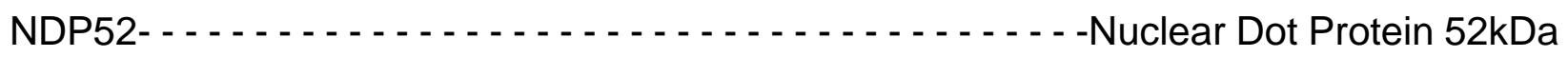

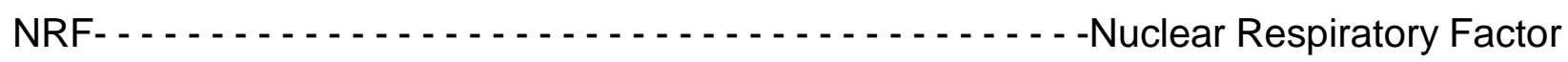

$\underline{0}$

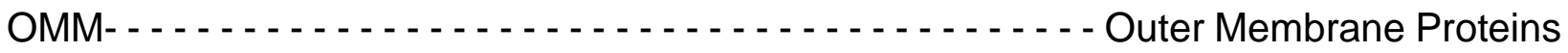

$\underline{\mathbf{P}}$

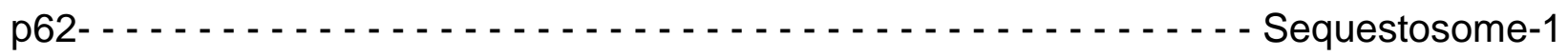

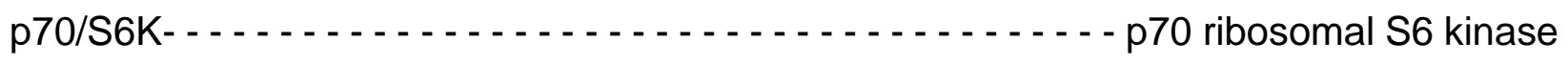

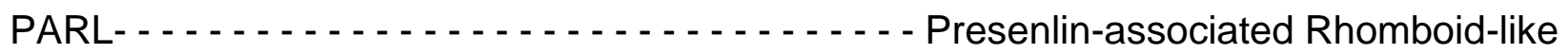

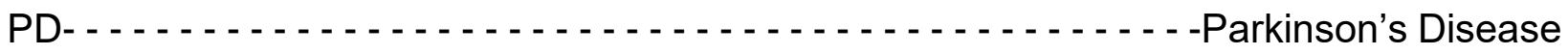
PE-Phosphatidylethanolamine PEPomegranate Extract PGC-1a- - - - - Peroxisome Proliferator-activated Receptor Gamma Co-Activator-1 Alpha $\mathrm{PI} 3$ -Class III phosphatidylinositol

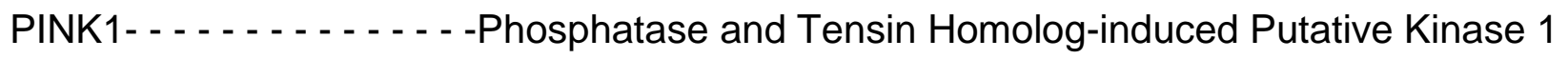

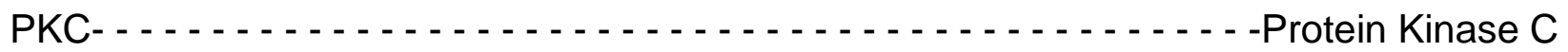
PLEKHM1 - - . - . - . - - -Pleckstrin homology domain-containing family M member 1 PolyQ $\ldots \ldots$ Polyglutamine PTM - 
$\underline{\mathbf{Q}}$

CytoQ-

Cytosolic Q Bodies

$\underline{\mathbf{R}}$

Rheb- . . . . . . . . . . . . . . . . . . . . . .

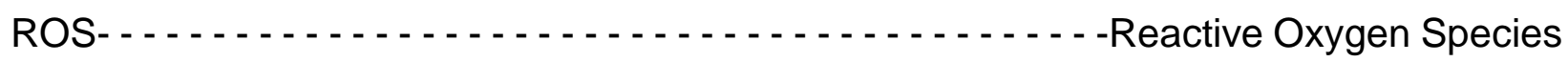

$\underline{\mathbf{S}}$

SCA $\ldots$

SIAH $\ldots \ldots$. . . . . . . . . . . . . . . . . . . .

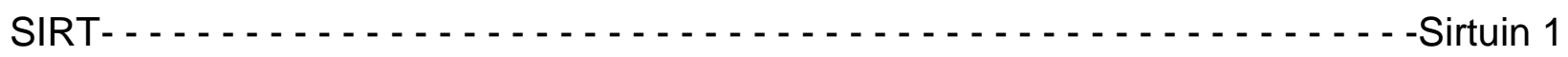

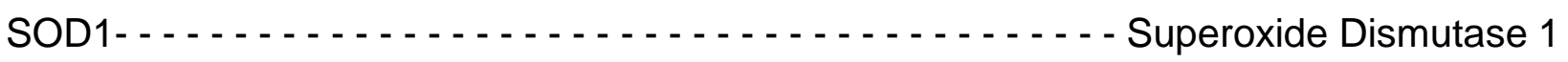

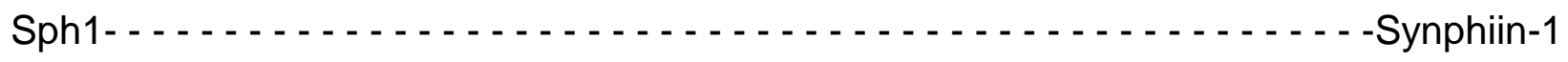

I

TDP-43 - . . . . . . . . . . . . . . . . . . . . . . . . . . .

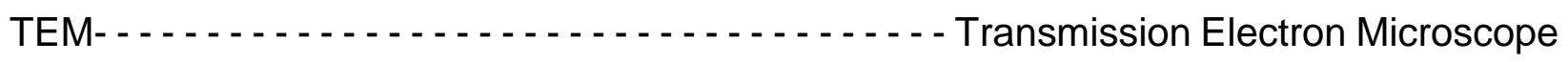

TFAM and TFB2M - . . . . . . . . . . . . . . . . . . . . . . .

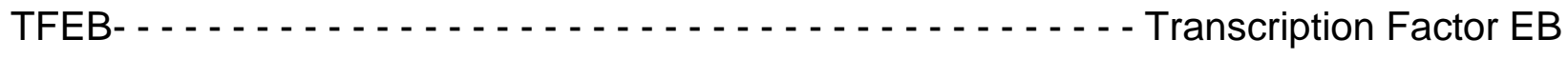

TOMM20 - - - - - - - - - - - Translocase of the Outer Membrane Mediated Protein 20

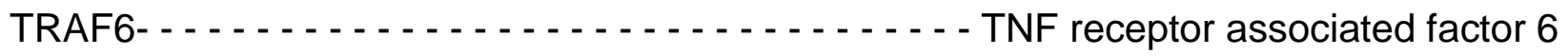

TRIC - . . . . . . . . . . . . . . . . . . . . .

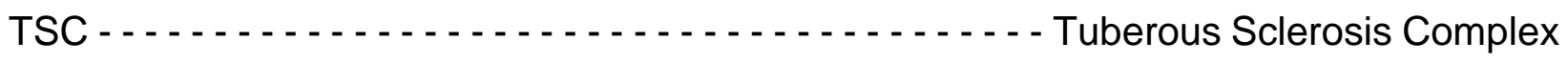

$\underline{\mathbf{U}}$

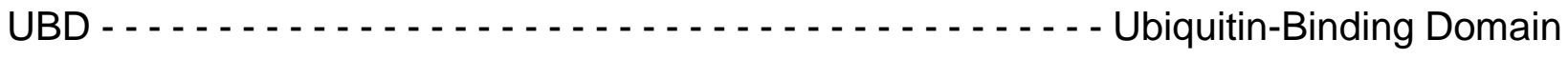




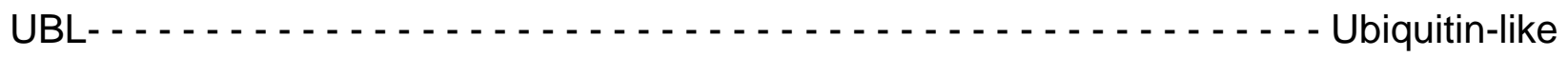

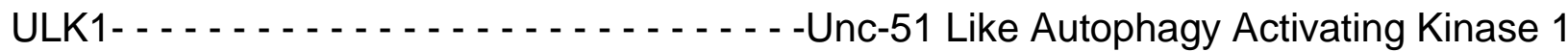

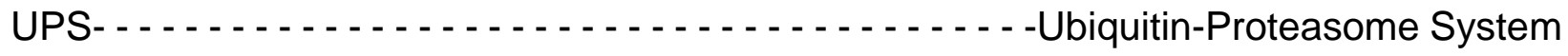
$\underline{\mathbf{v}}$

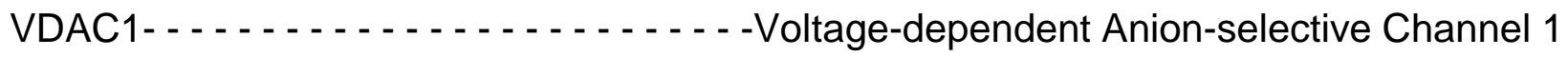

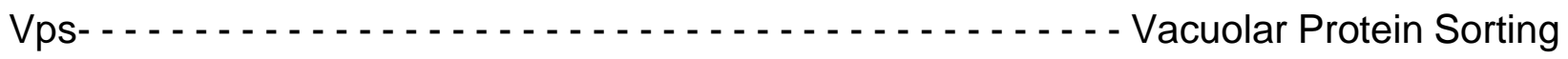

\section{$\underline{\mathbf{w}}$}

WIPI - - - WD-repeat Protein Interacting with Phosphoinositides 


\section{ABSTRACT}

Autophagy is an important quality control mechanism that degrades unwanted or damaged cellular components in the lysosomes to maintain cellular homeostasis. Autophagy was once thought as a non-selective catabolic process. However, it is now recognize that the cells can upregulate selective forms of autophagy to target specific cellular cargo for lysosomal degradation. Selective autophagy plays a major role in cellular stress resistance. Selective autophagic removal of pathogenic protein aggregates (aggrephagy) and damaged mitochondria (mitophagy) are crucial aspects of cellular physiology. Impairment in these processes is a common denominator underlying aging and neurodegeneration. Hence, this study aims to understand the mechanisms underlying efficient cargo recognition and handling in the selective removal of protein inclusions and dysfunctional mitochondria, and to derive ways to augment both processes to protect against cellular toxicity.

The first part of this study looked at post-translational regulation of aggrephagy. Synphilin1 (Sph1), a common component of protein inclusions in a-synucleopathies, has been shown to mediate different types of aggrephagy. Sph1 mediates constitutive turnover of small cytosolic aggregates (Agg) basally (basal aggrephagy), and facilitates formation and autophagic disposal of large perinuclear protein inclusion known as the aggresome (Agm). Sph1 is both ubiquitinated and phosphorylated. K63-linked polyubiquitination (K63U) of Sph1 promotes the stress-induced aggrephagy, but has no influence on the basal aggrephagy. The effect of phosphorylation on Sph1 aggrephagy activity remains unexplored; hence, the role of phosphorylation in influencing Sph1 basal and inducible aggrephagy was examined. In-silico and mass spectrometry analysis revealed Sph1 is highly phosphorylated, and chemical inhibition as well as genetic knockdown (KD) of PKC, CKII and GSK3 $\beta$ isoforms perturbed basal and inducible aggrephagy. These observations highlight phosphorylation as a molecular determinant for Sph1-mediated basal and inducible aggrephagy. Furthermore, inhibition of GSK3 $\beta$ reduced the recruitment and activation of E3 ligase Parkin to Sph1, as well as the levels of K63U linkages on Sph1 under proteasomal stress. This finding demonstrates the crosstalk 
between phosphorylation and ubiquitination, mediated by an interplay between GSK3 $\beta$ and Parkin, underlies the mechanism for stress-induced aggrephagy.

The next part of this study focused on the functional food, pomegranate, in the regulation of selective autophagy. The beneficial effects of pomegranate and its constituents to alleviate protein amyloid loads and enhance mitochondrial functions in-vivo were partially overlapped with autophagy induction. Hence, using a pomegranate extract (PE), the potential of pomegranate and its constituents to maintain proteostasis and mitochondrial health via upregulation of selective autophagy (aggrephagy and mitophagy) were examined. Our study demonstrates PE as a regulator of autophagy-lysosomal fitness. Furthermore, activation of transcription factor EB (TFEB) via the lysosomal- $\mathrm{Ca}^{2+}$ signaling underscores the mechanism for PE-induced autophagy. These findings highlight pomegranate as a novel activator of TFEB to upregulate autophagy. Further examination of the effects of PE-induced autophagy under proteotoxicity revealed that PE did not mediate the turnover of oxidized proteins or protein aggregates under proteasomal stress; instead, PE-induced autophagy enhanced the mitochondrial competence basally to facilitate mitophagy upon onset of mitochondrial stress. PE induced the formation of characteristic "donut-shaped" mitochondria, and promoted the recruitment of autophagosomes to the mitochondria under basal condition. Upon mitochondrial stress, PE preserved the mitochondrial health, and protected the mitochondria against reactive oxygen species (ROS)-induced redox toxicity by promoting PINK1-Parkin mediated mitophagy. KD of TFEB abrogated mitophagy induction and the protective effects of PE on the mitochondria. Hence, our study uncovered the mechanism underlying the beneficial effects of pomegranate and its constituents on mitochondrial health. PE activates TFEB to prime the mitochondria for clearance under basal condition, and promote selective autophagic clearance of damaged mitochondria under mitochondrial stress.

Collectively, our study uncovered novel autophagy mechanisms to facilitate the selective removal of protein aggregates and damaged mitochondria under physiological and pathological conditions. These understandings provide new avenues for enhancing 
aggrephagy and mitophagy to counteract proteotoxicity and mitochondrial dysfunction observed in aging and diseased states to promote health and longevity. 


\section{CHAPTER 1}

INTRODUCTION

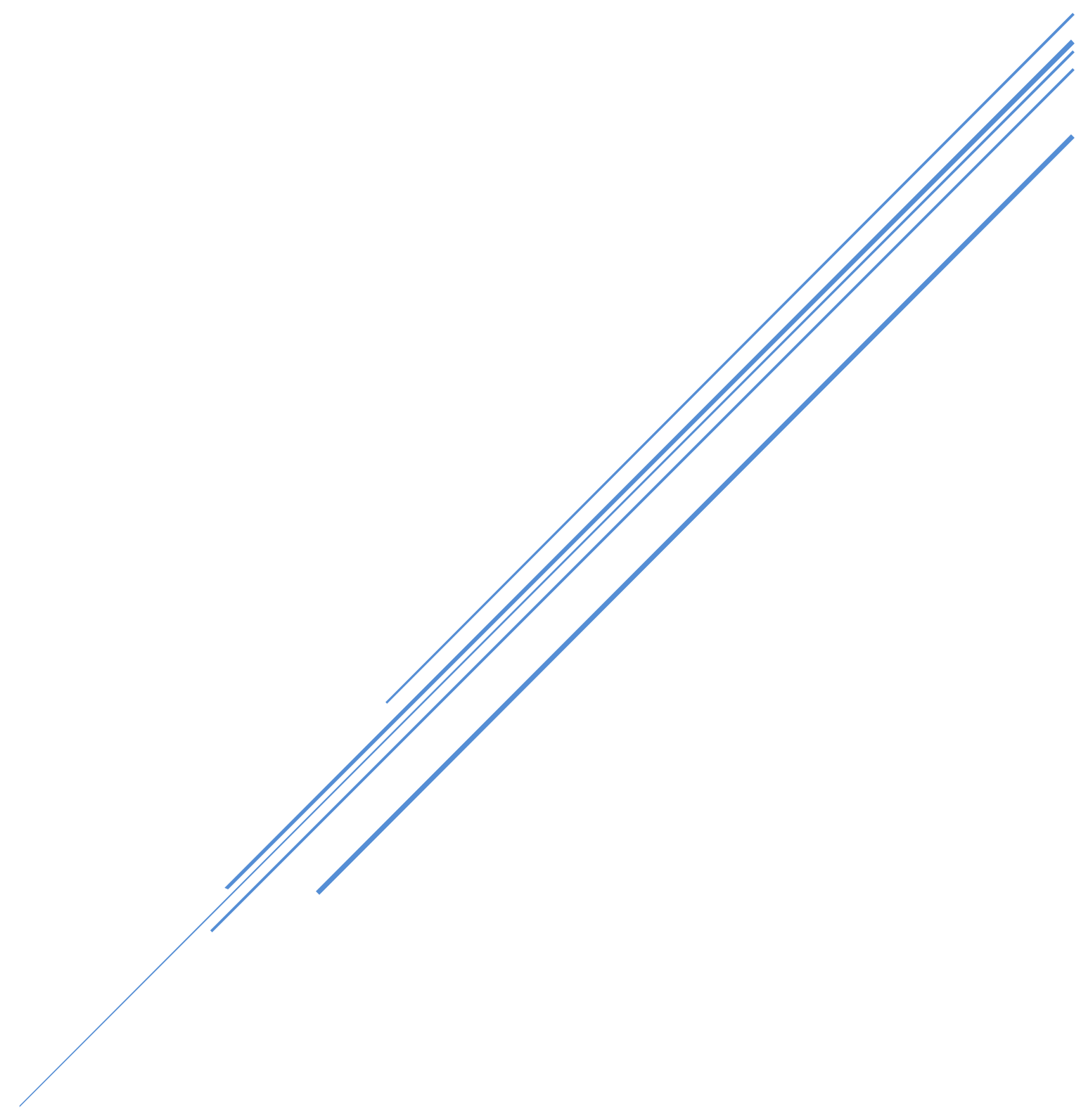




\subsection{OVERVIEW}

Intracellular processes are finely controlled and timely executed depending on the needs of the living cell. However, due to the complexity of the biosynthetic or signaling pathways, inherent mistakes can arise which require apt removal and/or repair of aberrant components to protect cellular physiology. In addition, extracellular stressors including physical, chemical or infectious insults could further aggravate the severity of the injuries and accelerate cell death [1]. To maintain cellular survival under adverse and changing intracellular conditions, a hierarchy of mechanisms are in place to monitor, identify, remove or repair intracellular damages. This network is termed as the cellular quality control system [1].

Quality control mechanisms constitute a crucial regulatory feature of cell's physiology. Disruption of cellular homeostasis due to declining efficiency of quality control machineries have been linked to aging and onset of diseases, including cancer and neurodegeneration [2]. The quality control system encompasses three different mechanisms- the first one involves the molecular chaperones that facilitate the repair of aberrant proteins [3], and the second mechanism involves the ubiquitin-proteasome system (UPS) that degrades non-essential or terminally damaged proteins [4]. However, these two quality control mechanisms only play a role in the repair or degradation of polypeptides. Large macromolecules, including protein complexes and organelles, are not amenable for repair by molecular chaperones and degradation by the UPS. Hence, the third quality control mechanism, macroautophagy, which involves degradation by the lysosome compartment, is important to handle the turnover of large macromolecules [5].

The lysosome was discovered in 1956 by Christian DeDuve and his colleagues, who observed appearance of membrane-bound lysosome structures that contained organelles like mitochondria under the transmission electron microscope (TEM). Since then, DeDuve has described the process whereby cells digest their own intracellular materials via the lysosomes (the "self-eating" process) as autophagy [6]. Thereafter, different types of autophagy degradation pathways have been characterized. These 
include the macroautophagy, microautophagy and chaperone-mediated autophagy (CMA) pathways. Although all three pathways involve delivery of substrates to the lysosome for degradation, they have distinct mechanisms and recognize different types of substrates [7]. The macroautophagy pathway, which we will henceforth term as autophagy, is the most extensively studied pathway, and targets a wide variety of substrates, including lipids, carbohydrates, proteins, organelles and invading pathogens, for degradation [8].

Although once thought as a non-selective bulk degradation process to recycle intracellular components to generate amino acids and ATP, autophagy is now recognized to selectively target specific substrates for lysosomal degradation under certain stress conditions [9]. To date, selective autophagy has been shown to recognize and degrade protein aggregates, damaged organelles including mitochondria, ribosomes and lysosomes, and invading pathogens [10]. The functional significance for such selectivity allows removal of a specific type of substrate without affecting the remaining intracellular constituents. For example, during proteotoxic stress, the selective removal of the protein aggregates refreshes the damaged proteome without perturbing the functional portion of the cellular proteome [11].

Selective autophagy has become a recent subject of rigorous study after several reports highlighted the involvement of selective autophagy impairment in aging and various human diseases [12, 13]. The autophagy pathway has thus, became an attractive therapeutic target. Many small molecule compounds have been discovered to modulate autophagy and mitigate disease progression [14]. Although promising, these compounds often serve to enhance the overall autophagic activity in the cell rather than modulating the specific degradation of substrates. Moreover, these small molecule compounds often elicit undesirable off-target side effects [15]. Hence, therapeutic strategies that target selective autophagy rather than the general autophagy may maximize the clinical benefit by enhancing selectivity and reducing off-target effects.

An emerging concept involving prevention or delay of the onset, rather than a reversion of the pathogenesis has gained traction in therapy against human diseases. Besides 
drugs, a more natural form of remedy involving dietary modulation as well as lifestyle changes might offer a safer therapeutic route to counteract diseases. Indeed, lifestyle practices, including caloric restriction (CR) and consumption of polyphenols-enriched food products like berries and red wine, has shown to enhance longevity and counteract diseases like neurodegeneration and cancer $[16,17]$. Interestingly, the mechanism that underlies the health benefits of these natural remedies seems to overlap with autophagy induction $[16,17]$.

In summary, in order to avoid autophagic overdrive, it is important to understand the mechanisms underlying selective autophagy to elucidate the key determinants that govern substrate selection for targeted lysosomal clearance. Furthermore, the potential of modulating selective autophagy through dietary intake for cellular health and mitigation of human diseases remains poorly explored. Hence, this study aims to decipher the selective mechanisms underscoring specific autophagic clearance of protein aggregates (aggrephagy) and damaged mitochondria (mitophagy). The first focus is on Synphilin-1 (Sph1), an aggregation-prone protein associated with Parkinson's disease (PD) but has also been shown to be an adaptor for facilitating aggrephagy [18]. We investigated how modification of Sph1 by phosphorylation and ubiquitination could regulate its ability to selectively target protein inclusions for lysosomal degradation. Next, the study examined the potential of polyphenols-enriched pomegranate extract $(\mathrm{PE})$ in modulating selective autophagy as a cytoprotective response against proteotoxicity and mitochondrial stress. By elucidating the mechanisms for selective autophagy mediated by Sph1 and PE, we hope to uncover new therapeutic targets and strategies to modulate selective autophagy to cope with the onset and progression of aging and human diseases. 


\subsection{CELLULAR QUALITY CONTROL MECHANISMS}

To understand the importance of quality control mechanisms in maintaining cellular homeostasis, we first review how damages to intracellular constituents could occur. Cellular insults can occur on three different levels: the genomic level (DNA and mRNA), the molecular level (protein) and the organelle level.

DNA level. At the DNA level, spontaneous damage may occur via chemical hydrolysis of the nucleotide bases including deamination, depurination, and depyrimidination. Induced damage of the bases can occur due to exposure to ultra-violet (UV) radiation, oxidation and chemical insults. These injuries can lead to different types of DNA damage, including formation of DNA adducts, mismatches and single or double-stranded breaks [19]. A diverse range of DNA repair mechanisms exist to cope with the different types of damages. For example, in eukaryotic cells, damaged or mismatched nucleotides can be excised and replaced by endonucleases and DNA polymerase, and double-stranded breaks can be repaired by homologous recombination and non-homologous end joining [20]. Loss of DNA surveillance to identify and rectify the damages will lead to alteration in protein function and cellular activity, and manifest as hereditary and genetic diseases [21].

mRNA level. The regulated turnover of mRNA is important as it controls protein expression and specifies the entire proteome of the cell. In eukaryotic cells, damages at the mRNA level often occur due to defects in transcripts processing, such as splicing, 5' RNA capping and polyadenylation [22]. Defective mRNA can be degraded via two mechanisms, both initiated by the shortening of the polyadenylation tail at the 3' end [23]. In human cells, mRNA degradation can also be initiated by addition of a polyuracil tail [24]. As mRNA quality is closely linked with protein synthesis, failure to detect incorrectly processed mRNA and the presence of redundant transcripts contribute to translation of truncated or elongated polypeptides that are inactive. 
Protein level. Proteins are the most structurally versatile form of macromolecules and play crucial roles in all biological processes. In eukaryotic cells, over ten thousand protein species specify the cellular proteome [25]. Protein homeostasis, or proteostasis, is a process that regulates the quantity as well as the quality of newly synthesized proteins. Appearance of damaged proteins is a consequence of various factors, including mutations or abnormal post-translational modifications (PTM) that compromise the proper protein-folding dynamics and/or alter the native protein behavior [26]. Often, these nonnative protein conformers have elevated hydrophobicity and may interact with intracellular macromolecules or organelles and perturb cellular activity [26]. Proteostasis is achieved by an integrated network of several hundred proteins involved in folding, transport and clearance of proteins, all of which are critical to prevent accumulation of misfolded and defective proteins [27]. The process can be constitutive to control the lifecycle of proteins under physiological conditions, but can also be induced in the presence of cellular stressors that compromise protein functions [28]. Proteostasis consists of three lines of mechanism: (1) Molecular chaperones that facilitate refolding of non-native protein conformers; (2) Degradation of terminally misfolded proteins by the UPS; (3) Degradation of large protein inclusions by the autophagy pathway. Loss of these protein quality control pathways is associated with appearance of intracellular protein aggregates, a phenomenon observed in neurodegenerative conditions and aging [29, 30].

Organelle level. Organelles are important regulatory compartments in the cell for localized control of specific catabolic and anabolic processes that support overall cellular homeostasis and survival. Hence, the turnover of aged and damaged organelles is important in maintaining cellular physiology [31]. Organelle homeostasis is, in fact, closely linked with protein turnover. An organelle can be equated to a multicomplex structure with a membranous compartment made up of lipids and proteins that carry out the metabolic processes. Thus, aberrant proteins will compromise the organelle's biological functions. Autophagy represents an important pathway for removal of dysfunctional organelles, and impairments in the turnover of these damaged intracellular components contribute to aging and development of diseases [32]. Notably, autophagic degradation of 
mitochondria, termed mitophagy, is currently gaining attention because of its role in the pathogenesis of neurodegenerative disorders and aging [33].

In the next section, we will review the three lines of cellular quality control mechanisms initiated by the (1) molecular chaperones, (2) UPS and (3) autophagy pathway, and how they can cope with aberrant accumulation of damaged proteins and organelles.

\subsubsection{Molecular chaperones in protein folding and proteostasis}

Molecular chaperones are proteins that can interact, stabilize and assist another macromolecule to acquire its functionality without themselves being part of the final structure [34]. In proteostasis, chaperones take on the first line of defense by keeping their substrates in their proper native conformation. They facilitate proper folding and maturation of nascent polypeptide chains newly synthesized from the ribosome, and help in refolding of misfolded proteins [34]. Other functions of chaperones in protein homeostasis have been reported, including protein trafficking and translocation, and assistance in proteolytic degradation of terminally misfolded proteins by the UPS and lysosome [3, 34]. Molecular chaperones are often known as heat shock proteins (Hsp). Their expression can be constitutive to perform vital housekeeping functions, or can be upregulated in the presence of stressors like heat or other forms of stimuli that promote protein denaturation. Chaperones are classified per their molecular weight, and the Hsp involved in protein folding include Hsp70, Hsp90 and the chaperonins (Hsp60) [34].

Hsp70. Hsp70 is a central player in protein folding and proteostasis. It consists of two domains, a $\mathrm{N}$-terminal nucleotide binding domain and a $\mathrm{C}$-terminal substrate binding domain [35]. Hsp70 does not function alone, and requires the presence of other cofactors including the co-chaperone, Hsp40, and nucleotide-exchange factor to facilitate binding and release of protein substrates [35, 36]. Briefly, the mechanism involves switching of Hsp70 from a low affinity to a high affinity state, which cycles to bind and release the polypeptide substrate to facilitate folding. The released polypeptide can rapidly fold and bury their hydrophobic resides, or rebind to Hsp70 for conformational remodeling to 
achieve a more stable state [37]. The Hsp70 folding mechanism involves fast-folding kinetics. Proteins that are unable to be properly folded by Hsp70 are transferred to Hsp60 chaperones, known as chaperonins to allow for slower folding kinetics [38].

Hsp60 or chaperonins. The chaperonins can be further subdivided into two groups. In eukaryotic cells, the group I chaperonins are known as the Hsp60, and the group II chaperonins are known as the $\mathrm{T}$ complex protein ring 1 complex/chaperonin containing TCP1 (TRIC/CCT). Hsp60 are "folding cages" that enclosed unfolded proteins and allow their refolding inside the confined space. The steric confinement of the folding cage probably modulates the free-energy folding landscape of the unfolded protein intermediate, and remove the free energy barriers to allow it to fold into its native state [39]. The group II chaperonin acts in similar principle as the group I chaperonin cage, but encapsulates unfolded protein intermediates longer for refolding [40]. An example of Hsp60 substrate client is the polyglutamine-expanded huntingtin (Htt)- TRIC/CCT has also been shown to modulate the aggregation state of the Htt protein and convert them to non-pathogenic forms [41-43].

Hsp90. Hsp90 operates downstream of Hsp70 and facilitates late stage folding which is important for structure maturation. Hsp90 thus plays a more important role in the generation of functionally active protein conformers, with many of its clients being kinases and transcription factors [44, 45]. Similar to Hsp70, Hsp90 does not act alone but requires the presence of cofactors to regulate its activity. The mechanism by which Hsp90 and its cofactors regulate protein maturation involves binding of the ATP molecule and the inactive protein substrate to Hsp90, after which the Hsp90 undergoes conformational change accompanied by ATP hydrolysis to activate the substrate. In this mechanism, the cofactors help to modulate the kinetics of the process as well as substrate release from Hsp90 [46, 47]. 


\subsubsection{Ubiquitin-proteasome system (UPS)}

Whereas protein chaperones represent the first line of defense in the maintenance of proteostasis by facilitating proper folding and assembly of newly synthesized polypeptides, terminally misfolded proteins that escape the surveillance of molecular chaperones are targeted for degradation by the UPS [13]. Basally, the UPS system is also responsible for rapid turnover of short-lived proteins involved in transcriptional control or signal transduction pathways [14]. For UPS degradation, cellular proteins are targeted to the proteasome by ubiquitination, a form of post-translational modification (PTM).

Ubiquitin is a 76-amino acid molecule that is highly conserved among eukaryotes [15]. For a protein substrate to be directed to the proteasome for degradation, the former will undergo an ATP-dependent enzymatic cascade orchestrated by the E1 ubiquitinactivating enzyme, E2 ubiquitin-conjugating enzyme and the E3 substrate specificubiquitin ligase. In this process, the ubiquitin is covalently attached to the protein substrate via an isopeptide bond formed between the glycine residue located at the Cterminal end of the ubiquitin with the $\varepsilon$-amino group of a lysine residue within the protein substrate [48]. The ubiquitin molecule consists of 7 lysine $(K)$ residues which can be further attached to more ubiquitin molecules. Repeated rounds of further attachment of ubiquitin molecule to the previously attached moiety generate extended ubiquitin chains in a process known as polyubiquitination. Depending on which lysine residue in the ubiquitin is further conjugated to extra ubiquitin, different polyubiquitination linkages could be generated, such as K48-linked polyubiquitination (K48U), K63-linked polyubiquitination (K63U) etc. [16].

The length and the type of ubiquitin chain determine the degradation fate of a protein substrate. Notably, K48U linkage is an important signal for directing protein substrates to the UPS for degradation [18]. It appears that the specific recognition of K48U substrates by the proteasome is due to the precise structural architecture of the substrate recognition site at the $19 \mathrm{~S}$ regulatory subunit, which can accommodate the closed conformation of the K48U [19-21]. K11-linked polyubiquitination (K11U), which also adopts a similar 
closed conformation as $\mathrm{K} 48$, has also been reported to facilitate targeting of protein substrates to the UPS [22]. Over the recent years, it has also been suggested that mono or multi-mono ubiquitination could similarly target proteins for UPS degradation [18, 23]. It appears that shorter protein polypeptides that are less than 150 amino acids in length were modified by monoubiquitination, whereas bigger substrates are modified by polyubiquitination to be directed for proteasome degradation [24].

Although the proteasome is an important proteolytic compartment to remove damaged proteins, the small diameter of the $20 \mathrm{~S}$ catalytic core can only accommodate substrates up to $1.0-1.5 \mathrm{~nm}$ wide [25]. Protein inclusions are therefore not amenable for degradation by the UPS. Moreover, these large protein inclusions have been shown to impede the proteasome function by binding and blocking the substrate entry gate at the 19S regulatory subunit, creating a vicious cycle of proteasome impairment with further protein aggregation [26-28].

\subsubsection{Autophagy}

The lysosome is a major proteolytic organelle in the cell that can accommodate large protein inclusions as well as organelles. The removal of intracellular constituents by the lysosome compartment is termed autophagy [11]. Three major types of autophagic pathways have been characterized in mammalian cells: (1) Microautophagy, (2) CMA and (3) Macroautophagy (Fig. 1.1). The three different autophagic pathways can act simultaneously or synergistically to maintain intracellular homeostasis, although they differ in their molecular machineries as well as the types of cargo degraded.

Microautophagy. Microautophagy is a lysosomal degradation process that involves sequestration of intracellular constituents by invagination of the lysosomal membrane, and subsequent engulfment of substrates into the lysosomal lumen for degradation. Of the three types of autophagic processes, microautophagy is the least characterized pathway in terms of the mechanism and cargo selectivity in mammalian cells [49]. However, investigations in yeast have unraveled the molecular machinery of the 
microautophagy cascade. Briefly, the molecular basis for this machinery involves: (1) Invagination of vacuole/lysosomal membrane to form tubular structure which sequesters cytosolic constituents, (2) deformation and expansion of the invagination to form a prevesicular structure that engulfs the constituents, (3) sealing of membranes and scission of vesicle from the vacuole/lysosomal membrane into the lumen and (4) degradation of the vesicle and the sequestered components by lysosomal hydrolases $[49,50]$.

Microautophagy in mammalian cells is characterized as a constitutive, non-selective process to degrade soluble cytoplasmic components for maintenance of intracellular homeostasis [50]. However, in yeast, selectivity in microautophagy has been observed to target piecemeal nucleus (non-essential portion of the nucleus) (micronucleophagy) [51] and mitochondria (micromitophagy) [52] for degradation. Deregulation of microautophagy has been implicated in human diseases. For instance, defective glycogen sequestration into the lysosome by microautophagy in skeletal muscle cells has been implicated in the pathogenesis of Pompe disease [53].

CMA. CMA represents a selective mechanism for degradation of soluble proteins by the lysosomes. The selectivity is conferred by the recognition of substrates that bear the CMA-targeting motif, defined by the loose pentapeptide KFERQ or a biochemically related KFERQ-like sequence, by a complex of chaperones and co-chaperones. These then shuffle the cytosolic proteins to the lysosome for degradation $[54,55]$. Briefly, the molecular mechanism of CMA can be divided into 4 distinct steps: (1) The protein substrate bearing the CMA-targeting motif is recognized by heat shock cognate protein of $70 \mathrm{kDa}(\mathrm{Hsc70})$ along with other co-chaperones Hsp40, Hsp90, Hsc70-interacting protein (Hip), Hsp70-Hsp90 organizing protein (Hop) and BCL2-associated athanogene 1 (Bag1) [56, 57]; (2) The Hsc70/co-chaperones assists the translocation of the protein substrate to the lysosomal membrane for binding to the cytosolic tail of the lysosomalassociated membrane protein type 2A (LAMP-2A) [58]; (3) Binding of the LAMP-2A to the substrate complex will promote LAMP-2A multimerization and substrate unfolding to facilitate translocation of the protein across the LAMP-2A receptor translocon [59]; (4) 
Once inside the lysosomal lumen, the protein substrate is degraded by the lysosomal proteolytic enzymes.

Basal CMA activity has been detected in mammalian cells, but has been shown to be upregulated under cellular stress caused by nutrient starvation [60] or oxidative stress [61] to supply amino acids for protein synthesis or remove damaged or aggregation-prone proteins respectively. Impairment of CMA has been linked to the pathogenesis of neurodegenerative diseases such as PD due to the failure to remove aggregated or misfolded proteins like $\alpha$-synuclein and leucine-rich repeat kinase 2 (LRRK2) mutants [62, 63]. In light of this, upregulation of CMA is a viable therapeutic option against neurodegenerative diseases. For example, overexpression of LAMP-2A in neuronal cells has been shown to mitigate $\alpha$-synuclein and Htt-induced neurotoxicity in neuronal cells and Huntington's disease (HD) mouse model by promoting their removal $[64,65]$.

Macroautophagy. Macroautophagy (henceforth termed autophagy), is a lysosomal degradation pathway which can dispose of large macromolecules, including protein inclusions, damaged organelles and invading microbes. This degradation pathway is highly conserved from yeasts to animals, with both sharing the same core autophagic machinery [8]. Briefly, macroautophagy involves sequestration of autophagic substrates into a double-membrane vesicle termed the autophagosome, and subsequent fusion of the autophagosome with the lysosome to degrade the constituents [66].

Whereas basal autophagy has been observed to maintain cellular homeostasis, the process can be upregulated upon cellular stress like starvation to degrade intracellular components in order to generate amino acids and energy [67]. Autophagy can be highly selective, targeting only specific substrates for degradation. Impairment selective autophagy has been shown to underlie the pathogeneses of various human diseases, particularly of neurodegeneration [32].

For this thesis, we will be focusing on the selective autophagy pathways that specifically degrade protein inclusions (aggrephagy) and damaged mitochondria (mitophagy). In the 
subsequent sections, we will review the core autophagy machinery in mammalian cells followed by the mechanisms defining selective degradation of proteins and mitochondria. 


\section{(C) CMA}

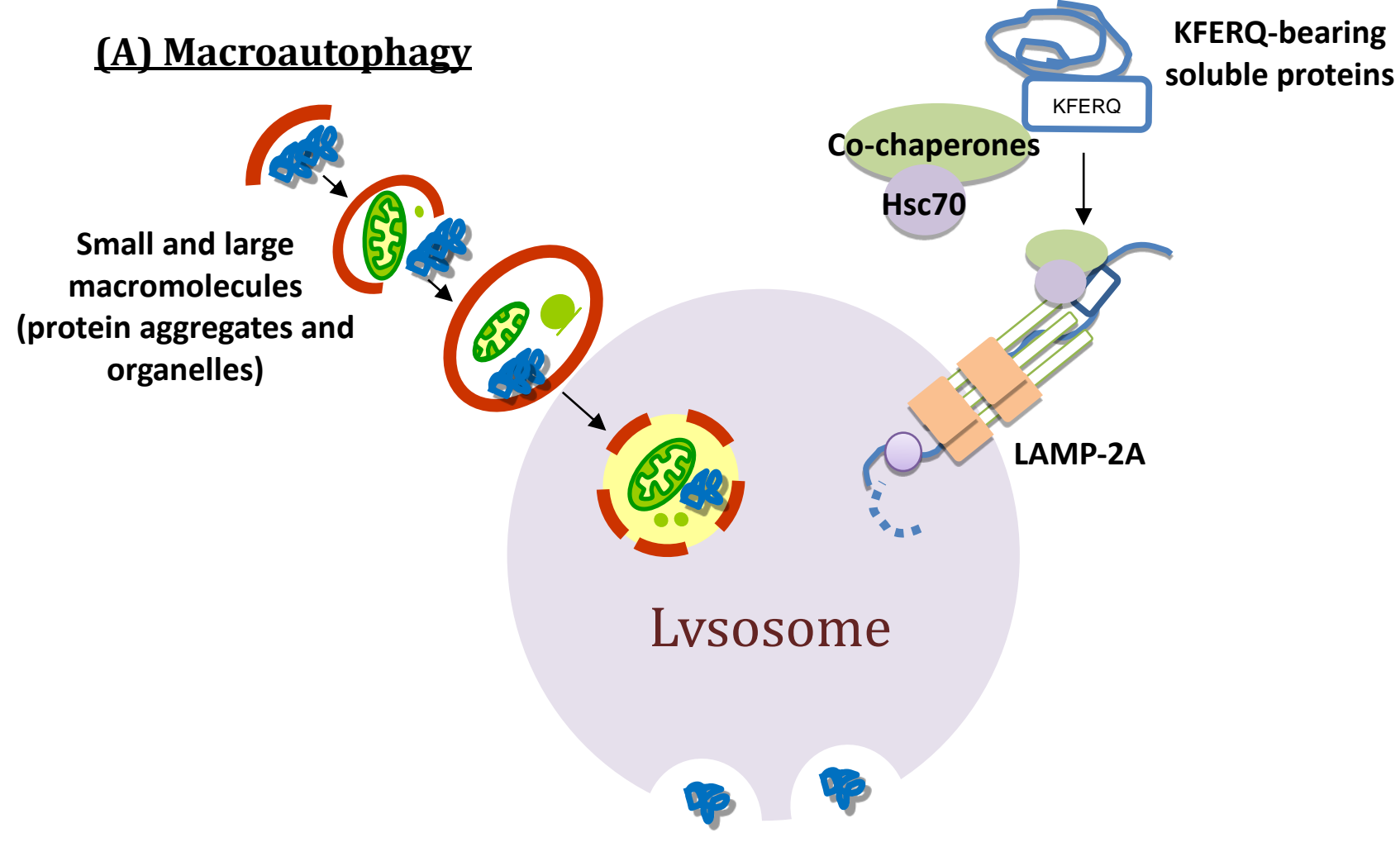

Small soluble proteins

(B) Microautophagy

Figure 1.1 The autophagy pathways. (A) Macroautophagy involves sequestration of substrates into autophagosomes compartment, followed by fusion of the latter with the lysosome. (B) Microautophagy involves sequestration of intracellular constituents (often small soluble proteins) by invagination of the lysosomal membrane, and subsequent engulfment of substrates into the lysosomal lumen for degradation. (C) CMA represents a selective mechanism for degradation of soluble proteins by the lysosomes. The selectivity is conferred by the recognition of substrates bearing the KFERQ-like CMAtargeting motif by the Hsc70/co-chaperones complex for delivery to the lysosomal membrane. The substrate-chaperone complex binds to the cytosolic tail of LAMP-2A. The substrate will subsequently be unfolded and translocate across the LAMP2A receptor. Once inside the lysosomal lumen, the protein substrate is degraded by the proteolytic enzymes. 


\subsection{THE AUTOPHAGY MACHINERY}

\subsubsection{The core autophagy machinery}

The autophagy machinery in mammalian cells can be subdivided into several distinct steps, which include (1) phagophore or omegasome (pre-autophagosome membrane) formation, (2) expansion of the pre-autophagosome membrane to form the autophagosome and (3) fusion of the autophagosome with the lysosome (Fig. 1.2). These steps are orchestrated by a group of proteins encoded by the autophagy-related genes (Atg) identified in yeast. These Atg genes have their corresponding homologues in mammalian cells [68-70].

Initiation. The very first step in autophagy requires the initiation of the formation of a membrane structure that can expand and grow around the autophagic substrates to form the mature autophagosome. In mammalian cells, this early membrane structure is known as the omegasome or phagophore. Whereas the omegasome originates from the endoplasmic reticulum (ER), the origins of the phagophore remain debatable, although several studies suggest that the golgi apparatus, endosomes or plasma membrane could contribute to the structure [71]. The initiation of the pre-autophagosome formation requires recruitment of the Unc-51 like autophagy activating kinase 1 (ULK1) complex consisting of ULK1/2, FAK family kinase-interacting protein of $200 \mathrm{kDa}$ (FIP200), Atg13 and Atg101, along with the class III phosphatidylinositol 3 (PI3)-kinase complex consisting of Beclin-1, Atg14, vacuolar protein sorting 34 (Vps34) and Vps15 to the site of origin for the pre-autophagosome membrane [72-74]. Localization of these ULK1 and PI3-kinase complexes will promote recruitment of the WD-repeat protein interacting with phosphoinositides (WIPI) proteins, essential for elongation of the pre-autophagosome membrane [75].

Elongation and autophagosome maturation. The localization of WIPI proteins to the pre-autophagosome membrane structure further recruits the Atg12-5-16 complex to 
trigger a ubiquitination-like reaction for elongation of the membrane [76]. The Atg12-5$16 \mathrm{~L}$ complex promotes conjugation of the microtubule-associated protein $1 \mathrm{~A} / 1 \mathrm{~B}$-light chain 3 form II (LC3-II) to the phosphatidylethanolamine (PE) on the pre-autophagosome membrane. Prior to this step, the LC3-II is generated from pro-LC3 from a cleavage and ubiquitination cascade involving Atg4, Atg7 and Atg3. Cleavage of the arginine residue at the C-terminal of pro-LC3 exposes a glycine residue that could conjugate to PE on the pre-autophagosome membrane. This process is facilitated by the ubiquitin-conjugating system involving E1 activating enzyme, Atg7, and E3 enzyme, Atg3. Conjugating of the LC3 protein to the pre-autophagosome membrane will drive the expansion and the closure of the structure to form the autophagosome [77]. Recent work has shown that the actin cytoskeleton is involved in shaping and bending of the pre-autophagosome structure to regulate the membrane curvature [78].

It is suggested that additional membrane sources could supply proteins and lipids for the elongation of the pre-autophagosome membrane. The golgi apparatus has been shown to be able to provide Atg9 transmembrane protein-enriched vesicles to the maturing autophagosome [79-81]. Membrane contact sites between the maturing autophagosome and other organelles like ER, golgi apparatus, mitochondria, endosome or lysosome could potentially facilitate exchange of membrane proteins and lipids between both compartments [82].

Autophagolysosome formation. This final step of the autophagic machinery underpins the degradative function of autophagy. The fusion between the autophagosome and lysosome to form the autophagolysosome has been shown to be regulated by late endosome protein Rab7A, and homotypic fusion and protein sorting complex (HOPS) proteins. The Pleckstrin homology domain-containing family M member 1 (PLEKHM1) acts as an adaptor to recruit the HOPS proteins to the autophagosome and lysosome to promote membrane tethering between both compartments [83]. Briefly, Rab7A is converted to its active GTP-bound form by the HOPS complex to facilitate membranes tethering. Meanwhile, soluble NSF attachment protein receptor (SNARE) protein syntaxin-17 will increase membrane permeability and induce membrane opening to 
facilitate fusion between the autophagosome and lysosome [84, 85]. Recently, it has been shown that Atg14, which is important for formation of the pre-autophagosome membrane, also plays a role in fusion between the autophagosome and lysosome. It is reported that Atg14 remains on the membrane of the mature autophagosome and interacts with syntaxin-17 to drive membrane tethering between the autophagosome and lysosome [86]. With the formation of the autophagolysosome, the substrates are degraded by the hyrolyases present in the lysosomal compartment. A study has demonstrated that the efflux of amino acids mediated by Atg22 from the vacuole (in yeast) or lysosomal compartment represents the final step in the autophagy cascade in eukaryotic cells. The released amino acids then provide the building blocks for maintaining protein synthesis [87].

\subsubsection{Regulators of autophagy induction}

Autophagy induction is controlled by cellular determinants that respond to changes in the intracellular energy and nutrient status [88, 89]. The mechanistic target of rapamycin (mTOR) complex 1 (mTORC1) and 5' AMP-activated protein kinase (AMPK) are two important kinases shown to regulate the activation of ULK1 and autophagosome biogenesis, and hence autophagy activity in response to low nutrients and ATP levels [88]. Autophagy has also been recently reported to be controlled at the transcriptional level via the transcription factor EB (TFEB) (Fig. 1.2). TFEB induces autophagosome and lysosome biogenesis under starvation to upregulate autophagy [90, 91].

Induction of autophagy culminates in increased metabolic robustness and cell survival. mTOR, AMPK and TFEB are key players for converging the energetic state signals to intricate tuning of the autophagic flux, resulting in increased ATP availability, improved energy efficiency and respiration to maximize cell viability [92]. For example, AMPK increases the metabolic capacity of autophagy under low energy charge condition (e.g. starvation), by stimulating autophagy to provide metabolites for ATP synthesis. Alongside with this, AMPK switches off ATP-consuming processes (e.g. protein synthesis) to reduce cellular energy demand, contributing to preservation of ATP pool [92]. Similar to AMPK, 
TFEB modulates cellular metabolic flexibility but via two distinct cellular avenues: (1) By enhancing biogenesis of the autophagic compartments and autophagy degradation capacity similar to AMPK; (2) By regulating mitochondria mass and health to influence respiratory capacity via mitochondria synthesis and clearance [92]. Hence together, mTOR, AMPK and TFEB connects metabolic demands to autophagy for energy control to preserve cell viability.

mTOR and AMPK. mTOR can exist as two different complexes: mTORC1 and mTORC2, depending on the interacting partners with mTOR [93]. Out of these two complexes, mTORC1 is implicated in autophagy modulation. mTORC1 negatively regulates ULK1 complex activation and inhibits autophagy [94, 95]. On the other hand, AMPK activates the ULK1 complex during condition of low nutrient levels to upregulate autophagy. It has been shown that the coordinated actions of mTORC1 and AMPK regulate ULK1 complex activity in response to changes in nutrient levels in cells [88].

Under physiological conditions where nutrients are abundant, mTORC1 is activated and phosphorylates ULK1 at S757 to disrupt AMPK and ULK1 interaction. This keeps the ULK1 complex inactive [96]. Activated mTORC1 can phosphorylate and activate downstream p70 ribosomal S6 kinase (p70/S6K) and eukaryotic initiation factor 4E binding protein (4BP1) to induce anabolic processes like ribosomal biogenesis and cell growth [97]. The abundance of branched chain amino acids like leucine can also activate mTORC1 to initiate protein synthesis [98]. However, once cellular energy is depleted, AMPK is activated to inhibit mTORC1 via phosphorylation of the tuberous sclerosis complex (TSC) and Ras homolog enriched in brain (Rheb) proteins located upstream of mTORC1 [99, 100]. As a result, mTORC1 dissociates from the ULK1 complex, and the concomitant reduction in the inhibitory S757 phosphorylation signal on ULK1 enables AMPK to interact with and phosphorylate the latter at S317 and S777 to activate it [96, 101]. The activated ULK1 complex can promote recruitment of the class III PI3-kinase complex to the site of the phagophore or omegasome to initiate autophagosome membrane biogenesis. 
TFEB. TFEB is a member of the microphthalmia family of the basic helix-loop-helixleucine-zipper (bHLH-Zip) transcription factors (MiT family). Transcription factors of the MiT family contain a basic region for DNA binding, HLH and Zip domains for dimerization, and a conserved activation domain important for transcription activation [102]. TFEB binds to the coordinated lysosomal expression and regulation (CLEAR) motif, a 10-base E-box-like palindromic sequence found on the promoter of lysosomal genes, and regulates transcription [103, 104]. In addition, TFEB is reported to bind to promoters of autophagy genes to induce autophagosome biogenesis and fusion between the autophagosome and lysosome [91]. Indeed, overexpression of TFEB has been demonstrated to increase the quantities of lysosomes and their proteolytic enzymes, as well as autophagic degradation of protein inclusions, lipids and damaged mitochondria $[91,105,106]$. In light of this, activation of TFEB represents a viable therapeutic target for lysosomal storage diseases (LSD), a group of disorders characterized by dysfunctional lysosomes due to deregulation of lysosomal $\mathrm{pH}$ and proteolytic enzymes [107]. Studies have shown that TFEB overexpression mitigates lysosomal dysfunction in various LSD mouse models [102, 108-110], and reduces accumulation of protein aggregates and alleviates neurotoxicity in PD [110-112], HD [91, 113], Alzheimer's disease and tauopathies (AD) mouse models [114-116].

The activation of TFEB is controlled by its intracellular localization, which in turn is regulated by phosphorylation events. Under nutrient-rich or physiological conditions, TFEB localizes to the cytosol and remains inactive. Upon starvation or stress to the lysosomes, TFEB rapidly translocate to the nucleus where it activates transcription of autophagy and lysosomal genes [90, 91]. Phosphorylation of TFEB by mTORC1 and mitogen-activated protein kinase 1 (ERK2) controls the cellular localization of TFEBphosphorylation at S142 and S211 inhibit translocation of TFEB to the nucleus, potentially by masking the nuclear localization signal on the transcription factor [117-119]. Recently, a third regulatory mechanism by the lysosomal $\mathrm{Ca}^{2+}$ has been shown to regulate the phosphorylation status on TFEB- starvation induces $\mathrm{Ca}^{2+}$ release from the lysosomal lumen through the $\mathrm{Ca}^{2+}$ channel mucolipion1, which activates the cytosol phosphatase calcineurin to dephosphorylate TFEB and promote TFEB nuclear translocation [120]. 
Activation of TFEB nuclear localization by these signaling cascades allows TFEB to enter the nucleus, and upregulate transcription of autophagy and lysosomal genes. 
(a) mTORC1-AMPK signaling
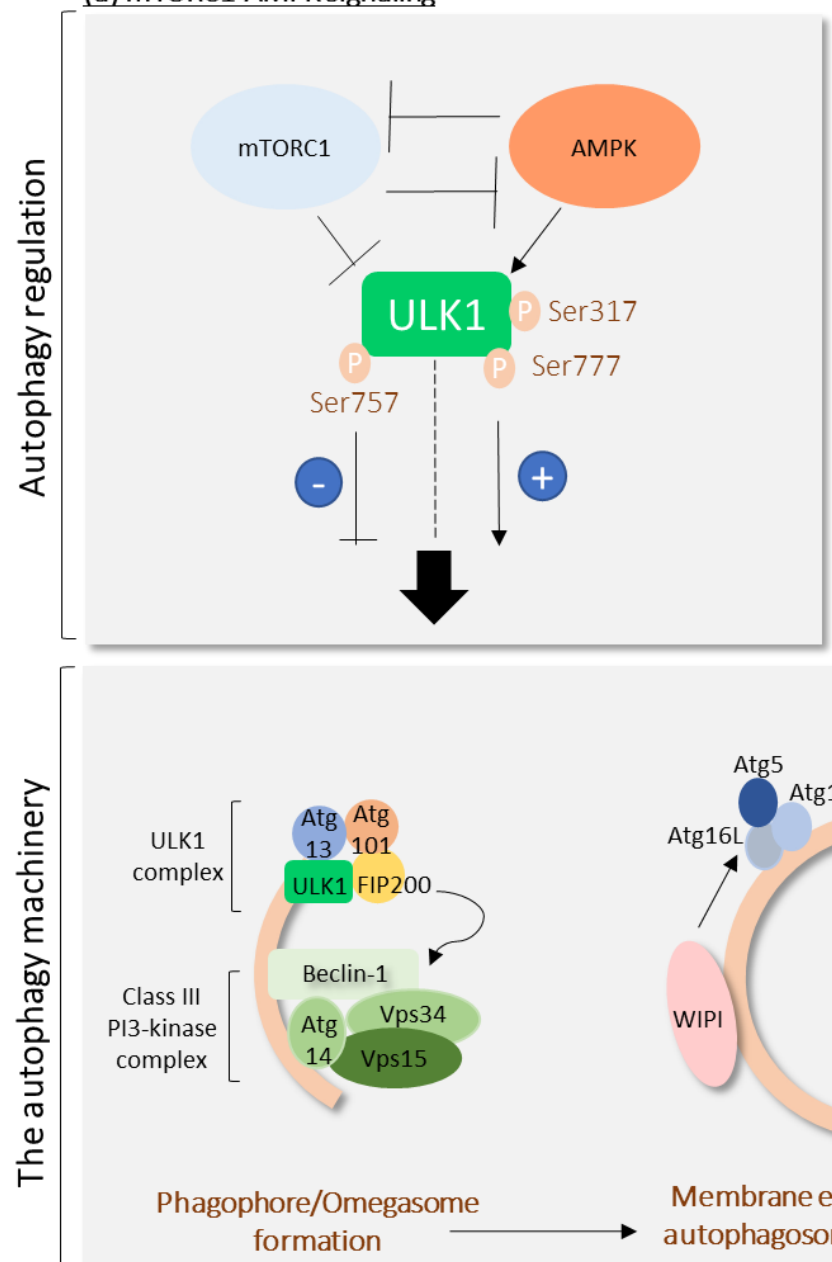

(b) TFEB signaling
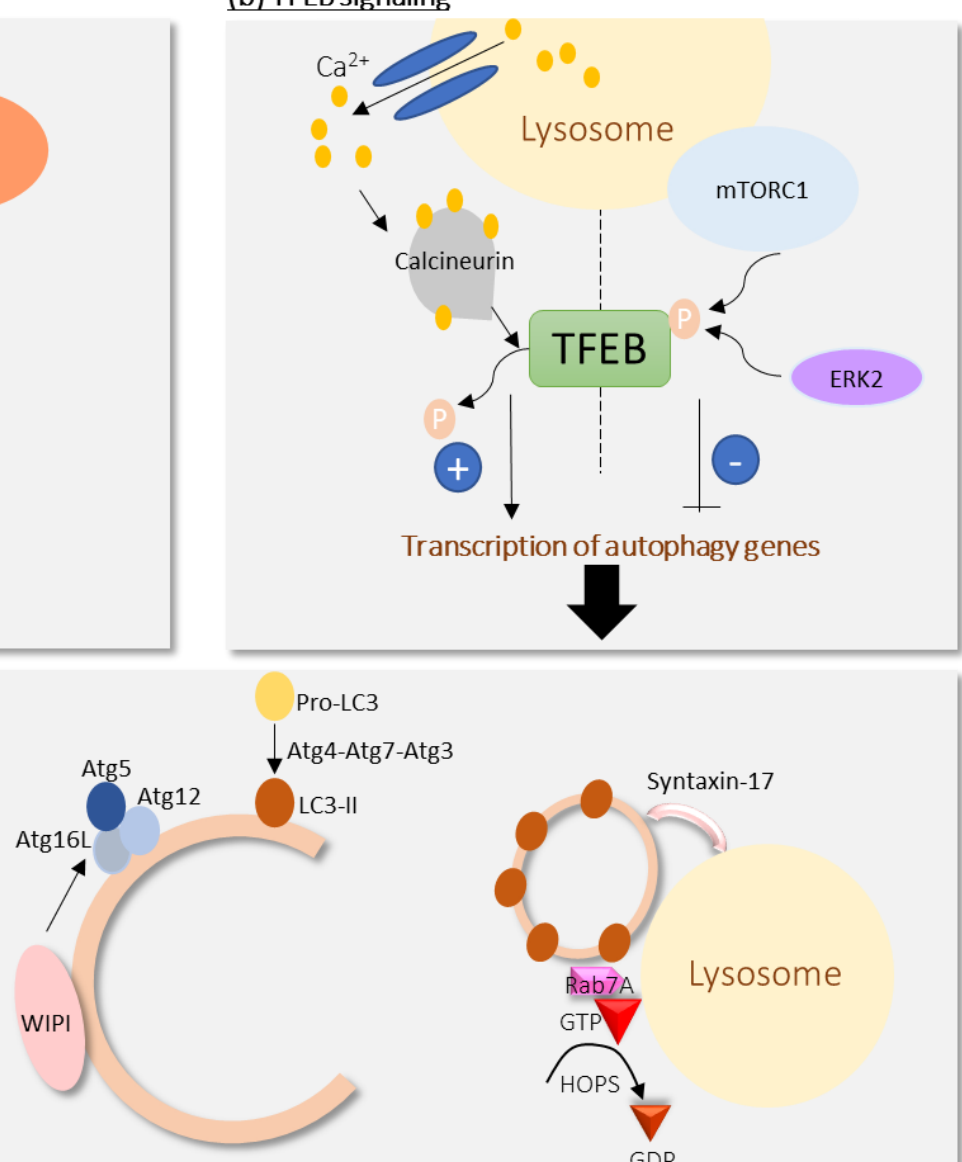

Membrane elongationand autophagosome maturation

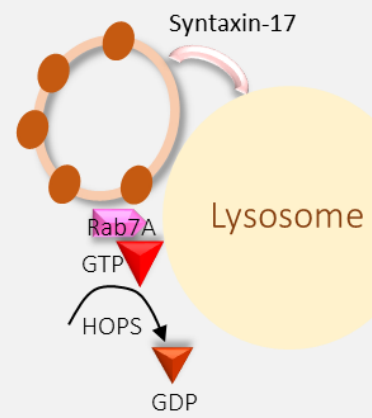

Fusion between autophagosome and lysosome

Figure 1.2 The autophagy machinery and the regulation by mTORC1, AMPK and TFEB signaling. (a) mTORC1 negatively regulates ULK1 complex activation and inhibits autophagy. Under physiological conditions where nutrients are abundant, mTORC1 is activated and phosphorylates ULK1 at S757 to disrupt AMPK and ULK1 interaction. During starvation, AMPK is activated to inhibit mTORC1. As a result, mTORC1 dissociates from the ULK1 complex and the concomitant reduction in the inhibitory S757 phosphorylation signal on ULK1 enables AMPK to interact with and phosphorylate the latter at S317 and S777 to activate it. (b) Phosphorylation of TFEB by mTORC1 and ERK2 at S142 and S211 inhibit translocation of TFEB to the nucleus and activate transcription of autophagy genes. A third regulatory mechanism by the lysosomal $\mathrm{Ca}^{2+}$ has been shown to regulate phosphorylation status on TFEB. Unlike mTORC1 and ERK signaling, 
starvation induces $\mathrm{Ca}^{2+}$ release from the lysosomal lumen through the $\mathrm{Ca}^{2+}$ channel mucolipion1 which activates the cytosol phosphatase calcineurin to dephosphorylate TFEB and promote TFEB nuclear translocation and transcription of autophagy genes. 


\subsection{THE AUTOPHAGY HIERARCHY AND SELECTIVE AUTOPHAGY}

\subsubsection{Basal and inducible autophagy}

Under physiological conditions, autophagy operates as a constitutive pathway dedicated to continuous recycling and renovation of intracellular constituents, including macromolecules like proteins, lipids and carbohydrates as well as organelles. This process is known basal autophagy, and it plays an important housekeeping role in maintaining cellular homeostasis [121]. A recent global analysis of the basal autophagy flux in primary human fibroblasts cells demonstrates that basal autophagy contributes to turnover of a substantial fraction of the proteome. Of these, the proteasome and CCT/TRiC chaperonin are robust substrates of basal autophagy [122].

The functional importance of basal autophagy is highlighted in several studies. Brainspecific ablation of the autophagy pathway via Atg5 and Atg7 knockouts in mice were shown to cause accumulation of ubiquitinated protein inclusions accompanied by appearance of behavioral features associated with neurodegeneration [123, 124]. Also, liver-specific inhibition of autophagy led to development of multiple liver tumors [125], and systemic inhibition of autophagy in mice results in neonatal lethality [67]. Atg5-deficient neonates also exhibited reduced plasma amino acid levels and displayed signs of energy depletion [67]. These studies not only highlight the importance of basal autophagy as a protective mechanism to prevent accumulation of damaged or redundant cellular constituents, but also underpin the metabolic role of autophagy for production of amino acids and energy homeostasis.

In addition to basal autophagy, autophagy can also be upregulated as an adaptive response to cellular stress. This process is known as the inducible autophagy pathway. The primary function of this pathway is to cope with starvation, where autophagy is upregulated for bulk removal of intracellular constituents to supply amino acids for ATP generation. However, it is now known that autophagy can be induced under stress 
conditions to selectively target specific cargo for degradation [121]. For example, upon proteasomal stress which leads to accumulation of pathogenic protein inclusions or mitochondrial stress that induces mitochondrial impairments, autophagy selectively targets the protein inclusions and damaged mitochondria respectively for clearance. Hence, selective autophagy can be considered a process that only specifically targets damaged or wanted constituents for autophagic clearance while not disturbing the remaining intracellular milieu and the housekeeping function of basal autophagy.

\subsubsection{Selective autophagy}

The selectivity of autophagy is controlled by specialized autophagy receptors known as cargo adaptor proteins. These adaptor proteins facilitate linking of the autophagic substrate to the autophagosome by simultaneously binding with the cargo and LC3 on the autophagosome membrane $[9,126]$. So far, more than two dozen cargo adaptor proteins have been identified in mammalian cells using yeast two-hybrid systems and proteomic approaches, and these have been shown to facilitate removal of protein inclusions/aggregates (aggrephagy), mitochondria (mitophagy), peroxisomes (pexophagy), ribosomes (ribophagy), proteasomes (proteaphagy), pathogens (xenophagy), zymogens (zymophagy), midbody rings and nucleic acids [9, 10, 127-131].

The cargo adaptors contain two important domains to allow simultaneous interaction with the autophagic substrates and the autophagosome. First, they contain a LC3 interacting region (LIR) that associates with LC3 on the autophagosome membrane. This motif, defined by the consensus sequence of Trp/Phe/Tyr-x-x-Leu/lle/Val (where $x$ can be any amino acid) is mapped from p62, the very first cargo adaptor protein characterized [127, 132, 133]. Since then, this motif has been used to search for other potential LC3interacting cargo adaptors proteins. Second, the cargo adaptor proteins contain an ubiquitin-binding domain (UBD) which interacts with the ubiquitin modifications on autophagic substrates. This underlies the fundamental role of ubiquitination as a signal tag on the autophagic substrate destined for selective autophagy [10]. Consistent with this notion, interplay between E3 ligases and deubiquitinase (DUB) has been shown to 
facilitate removal of several substrates, including protein inclusions, damaged mitochondria, intracellular pathogens and ribosomes. In addition, autophagic substrates have also been shown to be modified by various polyubiquitination linkages as well as monoubiqutination for recognition by cargo adaptor proteins to facilitate their autophagic removal [10].

To evaluate the inclusive list of selective autophagy processes currently in the literature will be beyond the scope of this thesis. Instead, we will focus on two types of selective autophagy that are most well-studied- aggrephagy and mitophagy. 


\subsection{PROTEOSTASIS AND AGGREPHAGY}

\subsubsection{Protein folding and misfolding}

Polypeptides are synthesized from translation of mRNA transcripts by the ribosomes as linear chains consisting of up to thousands of amino acids. These linear polypeptides exist in their non-native (unfolded) states and can only possess biological functions after being properly folded into their three-dimensional conformations [134, 135]. However, multiple factors can perturb proper protein folding to give rise to misfolded and damaged proteins (discussed in section 1.2). These faulty proteins often have enhanced hydrophobicity, which increase their propensity to aggregate and form protein assemblies.

\subsubsection{Protein aggregation and formation of inclusion bodies}

In a simple model, mutant, misfolded or damaged protein species can interact with one another and assemble into rope- or ribbon-like fibrillar oligomers containing extensive $\beta$ sheet structures (prefibrils). These intermediates are transient, and act as a focal point to trigger polymerization and association with other prefibrils to form insoluble protein aggregates known as amyloid fibrils [136]. An important progress in the field of amyloid assembly is the discovery of spatial propagation of the aggregation process within or between cells. Matured amyloids can fragment into multiple prefibrillar structures that can spread within or between cells to associate with the pre-existing pool of prefibrils. This will accelerate the spread of insoluble amyloid fibrils throughout the intracellular region or to other neighboring cells in a process similar to that of prion protein propagation [137139].

The appearance of neuronal protein deposits in diseases suggests that there is a close association between the large, insoluble amyloids and neurotoxicity [140]. However, a new perspective has emerged, which points to the prefibrillar intermediates that precede amyloid fibril formation as the primary pathogenic species responsible for neuronal insults 
[141-147]. The mechanism underlying the neurotoxicity is currently not well-established, but structural analysis of the different arrays of a-synuclein protein oligomers revealed that prefibrillar structures have larger hydrophobic surfaces and higher toxicity as compared to the matured fibril form. The high hydrophobic surface area to volume ratio of these intermediates could likely encourage aberrant interactions with proteinaceous and lipid intracellular components [145-147]. Meanwhile, sequestration of these prefibrillar structures into insoluble inert protein inclusions (a process termed as compartmentalization) has been shown to protect the intracellular environment from toxicity of the prefibrillar protein species [148].

\subsubsection{Compartmentalization as a cytoprotective mechanism against proteotoxicity}

Emerging evidences show that the cell actively transports aggregated protein species to designated sites, where they are confined into structures to protect the intracellular environment from the deleterious effects of the aggregates [149]. These cellular deposition sites represent quality control compartments to temporarily store harmful protein species before they are removed by the autophagy pathway. This pathway is evolutionary conserved, and has been observed in bacterial and eukaryotic cells [148156]

In yeasts, three types of inclusion bodies have been identified and characterized: (1) cytosolic Q bodies (CytoQ), (2) juxtanuclear quality control (JUNQ) and (3) insoluble protein deposits (IPOD) [152, 157, 158]. CytoQ are multiple cytosolic aggregates that appear upon heat stress, which are subsequently sequestered into the perinuclear JUNQ compartment $[159,160]$. The JUNQ is enriched with $26 \mathrm{~S}$ proteasomes and molecular chaperones, which suggests that the compartment might serve as a region to concentrate refolding of misfolded polypeptides, or enhance degradation of the terminally misfolded proteins. The IPOD in contrast, is free of proteasomes and molecular chaperones and localizes next to the vacuole. Its close vicinity to the vacuole suggests that the compartment serves as a staging area for bulk degradation of insoluble protein aggregates by autophagy [152, 158]. 
In mammalian cells, the aggresome (Agm) is the most well-characterized type of quality control compartment that shares striking similarities to Lewy bodies (LB) observed in $\alpha$ synucleopathies [161]. The Agm is formed in response to proteasomal stress, and involves retrograde transportation of ubiquitinated protein aggregates along the microtubules for sequestration at the perinuclear microtubule organizing centre (MTOC) region [148]. The spatial localization of the Agm to the perinuclear region has a functional significance. It is reported that during proteasomal stress, microtubule-mediated transport of lysosomes around the perinuclear region is reduced, creating an entrapment zone enriched with the proteolytic organelle. Hence, Agm formed at the perinuclear region are positioned near a large pool of lysosomes, which increases the propensity of lysosomal degradation of the protein aggregates [162].

They key to selectivity in the aggrephagy process is the ubiquitin modification on protein aggregates. Ubiquitination on protein aggregates is recognized by histone deacetylase 6 (HDAC6), and the latter interacts with the dynein-dynactin motor complex on microtubules to facilitate the transport process [155]. More specifically, protein aggregates that are modified by $\mathrm{K} 63 \mathrm{U}$ are preferentially selected for Agm assembly and subsequent autophagic degradation [163, 164]. E3 ligases and DUB are important molecular players for editing the ubiquitin code on protein substrates to facilitate aggrephagy. E3 ligases Parkin and TNF receptor associated factor 6 (TRAF6) are implicated in catalyzing K63U on protein aggregates to influence formation and clearance of Agm [165, 166]. Ataxin-3, an aggregation-associated DUB, cleaves away $\mathrm{K} 63 \mathrm{U}$ on protein aggregates and inhibits Agm formation [167]. It is recently reported that ataxin-3 also mediates generation of unanchored $\mathrm{C}$-terminal ubiquitin from polyubiquitin linkages on protein aggregates that can be recognized by HDAC6, suggesting an alternative pathway for selection of ubiquitinated protein aggregates for Agm formation [168]. With the formation of Agm, a series of events ensue to promote selective autophagic clearance of the protein inclusion. 


\subsubsection{The aggrephagy cascade}

Linking protein inclusions to the autophagosome. In mammalian cells, sequestosome-1 (p62) and neighbor of BRCA1 gene 1 protein (NBR1) are the most wellcharacterized adaptors that can link ubiquitinated protein inclusions to the autophagosome to facilitate selective autophagic degradation [169]. However, NBR1 and p62 engage different types of ubiquitinated protein aggregates. While p62 shows preferential binding to K63U [163, 170], NBR1 does not have selectivity against any type of ubiquitin linkages [171]. Structural differences in the UBD domain between p62 and NBR1 also account for much higher affinity of NBR1 for ubiquitin than p62 [171]. Despite the differences, p62 and NBR1 have been shown to act cooperatively to promote aggregation and selective degradation of ubiquitinated protein inclusions by autophagy [169]. In addition to linking protein inclusions to autophagosome, p62 has been shown to also initiate direct formation of the autophagosomal membrane around the protein inclusion. In this model, p62 interacts with the ULK1 complex at the pre-autophagosome structure to facilitate the recruitment of class III PI3-kinase complex for autophagosome biogenesis [172, 173].

Fusion between autophagosome and lysosome. The fusion process involves remodeling of the F-actin-cortactin network by HDAC6 [174]. p62 in the protein inclusion plays a role in this step by recruiting and enriching the perinuclear region with F-actin network assemblies for remodeling [175]. Microtubule-mediated transport of lysosomes within the perinuclear region is also reduced to increase the lysosomal population in the region for efficient fusion with autophagosomes [162]. Tethering between the autophagosome and lysosomal membrane prior to fusion has been described in section 1.3.1. The process is mediated by Rab7A, HOPS and syntaxin-17 [84, 85].

Agm disaggregation. In mammalian system, emerging evidence now shows that the Agm structure is disassembled prior to degradation by the autophagy pathway. Proteasome-associated DUB Poh1 cleaves off K63U chains from the Agm, releasing unanchored K63U chains that activate HDAC6 and promote cortactin-dependent F-actin 
remodeling to facilitate autophagosome and lysosome fusion [176]. This process requires the dissociation of the $20 \mathrm{~S}$ proteasome subunit from the Agm, and is mediated by the molecular chaperone Hsp90 [177].

\subsubsection{Regulation of the aggrephagy cascade}

PTM modulate the activities of cargo adaptors as well as HDAC6 to regulate selectivity in aggrephagy. Casein kinase II (CKII)-mediated phosphorylation of the UBD domain has been demonstrated to increase the binding affinity between p62 and ubiquitinated protein aggregates to enhance formation and degradation of inclusions [178]. On the other hand, glycogen synthase kinase 3 (GSK3)-mediated phosphorylation of NBR1 perturbs formation and clearance of ubiquitinated protein aggregates [179]. For the case of HDAC6, phosphorylation by CKII [265] and protein kinase C (PKC)-zeta [266] have been shown to modify the HDAC6 deacetylase activity and influence Agm formation as well as clearance.

\subsubsection{Non-canonical aggrephagy}

In addition to the ubiquitin and cargo adaptor proteins-mediated selective autophagy, recent studies have shown that selective autophagy can occur independently of both determinants for clearance of protein aggregates. Studies in the higher eukaryotes revealed that several cargo adaptor proteins are able to recognize and bind protein cargos independent of ubiquitination [10]. For example, optineurin, a cargo adaptor protein initially characterized to regulate selective degradation of ubiquitin-coated cytosolic Salmonella enterica, is shown to be able to directly recognize and degrade protein aggregates in an amyloid lateral sclerosis (ALS) zebrafish model via its C-terminal coiled-coil domain in a ubiquitin-independent manner [180].

Recently, a cargo adaptor-independent mechanism for clearance of protein inclusions has also been identified. The first evidence for the dispensability of cargo adaptors in 
aggrephagy came from the observation that Agm-like structures formed by PD-linked p38 and exogenously administered $\alpha$-synuclein are not able to recruit autophagy machinery despite enrichment with p62 adaptor proteins [181, 182]. Interestingly, when p38 is coexpressed with Synphilin 1 (Sph1), also a PD-linked aggregation-prone protein, Agm formed by p38-Sph1 can recruit autophagosome and lysosome compartments to facilitate clearance of the autophagy-resistant p38 inclusions [181]. Further examination revealed that Sph1 is still able to facilitate degradation of p38 inclusions under a cargo adaptordeficient environment [18]. This study highlights that Sph1 harbors an "aggrephagy promoting factor" that can promote clearance of protein inclusions independent of the activity of cargo adaptor proteins.

The ability of cells to evolve alternate selective autophagy machineries is particularly relevant in aging and neurodegenerative disorders, where p62 levels are perturbed [183]. The ability of Sph1 to promote aggrephagy independent of cargo adaptor proteins highlights that Sph1-mediated aggrephagy could serve as a compensatory mechanism to promote clearance of protein inclusions during aging and neurodegeneration. 


\subsection{SYNPHILIN-1: AN $\alpha$-SYNUCLEOPATHIES-ASSOCIATED PROTEIN IMPLICATED IN MODULATING CARGO ADAPTOR-INDEPENDENT AGGREPHAGY}

Sph1 is an aggregation-prone protein observed in LB in a-synucleopathies [184], a family of diseases which include PD, dementia with LB (DLB) and multiple system atrophy (MSA) associated with pathogenicity of $\alpha$-synuclein aggregates [185]. Sph1 is 919 -amino acid long and harbors four major domains: the ANK1 and ANK2 domains, a coiled-coil domain and an ATP-GTP-binding domain [186]. It is predominantly expressed in the brain, specifically in the substantia nigra, hippocampus and cerebellum [187], and is proposed to play a role in synaptic function and neurotransmission [188]. However, Sph1 functions are better and more often characterized under human pathological conditions, particularly in aging and neurodegeneration, where the expression levels are found to be elevated [189]. Studies have supported that the increase in the levels of Sph1 under pathological conditions could represent a cellular response aiming to protect against aggregateinduced neurotoxicity by facilitating formation of the Agm compartment and degradation of protein inclusions [186, 190-192].

\subsubsection{Synphilin-1 is robustly ubiquitinated and phosphorylated to regulate aggregation behavior}

Sph1 interacts with the neurotoxic $\alpha$-synuclein $[184,186]$, and this interaction has been shown to attenuate $\alpha$-synuclein-mediated neurotoxicity by promoting formation of cytoprotective LB-like Agm in PD-linked mammalian and yeast systems [190-194]. Indeed, a R621C mutation on Sph1 associated with familial PD, which results in loss of interaction between Sph1 and $\alpha$-synuclein, is linked with impaired Agm formation and stress-induced cell death [195]. These results highlight a role of Sph1 in suppressing proteotoxicity by compartmentalizing aggregation-prone proteins. 
The ability of Sph1 to form LB-like inclusions is modulated by ubiquitination and phosphorylation. Sph1 interacts with and is polyubiquitinated by several E3 ligases, including dorfin [196], seven in absentia homolog (SIAH) 1 and 2 [197], and Parkin [198, 199]. While the type of ubiquitin linkage catalyzed by dorfin is not known, SIAH mediates $\mathrm{K} 48 \mathrm{U}$ on Sph1 to target the protein for proteasome clearance while Parkin catalyzes K63U to promote Sph1 Agm formation. Interestingly, the K63U has also been shown to enhance autophagic susceptibility of Sph1 inclusions [18, 200].

In addition to ubiquitination by E3 ligases, phosphorylation of Sph1 by CKII and GSK3 $\beta$ also regulates Sph1 inclusion formation. CKII phosphorylation on Sph1 has been shown to reduce the interaction between $\alpha$-synuclein and Sph1 and hence inclusion formation [201]. On the other hand, GSK3 $\beta$ phosphorylates Sph1 at S556 to reduce the ubiquitination on Sph1 and inclusion formation [202]. This finding highlights that crosstalk between phosphorylation and ubiquitination modifies Sph1 aggregation propensity. Sph1 has also been shown to interact with the kinase LRRK2 to enhance inclusions formed between Sph1 and LRRK2. However, rather than influencing Sph1 phosphorylation, Sph1 reduces LRRK2 autophosphorylation and attenuates LRRK2 toxicity and dopaminergic neuron degeneration in Sph1 and LRRK2 double transgenic flies [203]. Phosphatase and tensin homolog-induced putative kinase 1 (PINK1) has recently been identified to interact with Sph1 [204]. However, PINK1 does not phosphorylate Sph1, and the interaction is more relevant to autophagic clearance of damaged mitochondria rather than protein inclusions [204].

\subsubsection{Synphilin-1 harbors Agm-targeting motif}

Whereas PTM has been shown to regulate Sph1 aggregation behavior, a report highlights that Sph1 harbors an intrinsic Agm-targeting motif that is necessary for the formation of Agm [205]. When overexpressed in-vivo, Sph1 forms two distinct types of protein inclusions: small, multiple cytosolic inclusions where they will be collectively termed as "aggregates" (Agg), and the single, large perinuclear Agm structure [205]. Under proteasome inhibition, Sph1 Agg sequester into the Agm structure, in line with the notion 
that proteasomal stress promotes Agm formation. In the report, it was found that Sph1 harbors Agg- and Agm-targeting domains that are important for formation of both types of inclusions. The ANK1 domain of Sph1 is responsible for formation of Agm, while the ANK2-coiled-coil domain is responsible for formation of Agg [205]. The ANK1 domain on Sph1 hence, represents a sensor for sequestering the cytosolic Agg into the Agm compartment in response to proteasomal stress. Interestingly, a recent study by our lab revealed that, in addition to the Agm-targeting property, the ANK1 domain stimulates autophagic clearance of inclusion bodies formed by PD-linked aggregation-prone proteins independent of cargo adaptor proteins p62 and NBR1 [18].

\subsubsection{Synphilin-1 ANK1 domain regulates autophagic susceptibility of Agg and Agm by basal and inducible aggrephagy}

The notion that Sph1 potentially harbors an "aggrephagy promoting factor" came from the observation that presence of Sph1 in autophagy-resistant inclusion bodies formed by PDlinked p38 protein renders the inclusion amenable to autophagic clearance by promoting recruitment of the lysosome compartments to the p38 inclusion [181]. Thereafter, our lab identified that the ANK1 domain of Sph1 is important and sufficient to mediate autophagic clearance of the Agg and Agm type inclusion bodies, by basal and inducible autophagy respectively [18]. Interestingly, the autophagic clearance of Agg and Agm is independent of cargo adaptor proteins p62 and NBR1 [18].

Upon closer examination, it was found that K63U on the ANK1 domain at Lys385 and Lys394 are critical for mediating autophagic removal of Agm. K63U reduces the mobility of the protein species on the surface of the Agm, thereby stabilizing the Agm structure to facilitate efficient recruitment of class III PI3-kinase complex to initiate autophagosome biogenesis directly around the Agm [18]. This highlights the potential of Sph1 being a novel aggrephagy promoting protein that links protein cargo to autophagosomes by inducing assembly of the autophagosome biogenesis machinery directly on the protein inclusion. The ability of Sph1 to promote cargo-adaptor independent aggrephagy mechanism suggests that upregulation of Sph1-mediated aggrephagy is a potential 
strategy that could compensate for cargo adaptor protein impairments in neurological diseases [12]. 


\subsection{AIM 1: UNDERSTANDING THE MECHANISM UNDERLYING SYNPHILIN-1 MEDIATED AGGREPHAGHY}

K63U on Sph1 ANK1 domain is important for the clearance of Agm by inducible aggrephagy. However, this modification is dispensable for the clearance of Agg by basal aggrephagy [18]. This data highlights two important key points: (1) the mechanisms regulating Sph1-mediated basal and inducible aggrephagy are different; and (2) understanding of the basal aggrephagy mechanism remains limited.

Emerging evidence shows that the cooperation between phosphorylation and ubiquitination regulates aggrephagy [10]. While ubiquitination serves as a tag for damaged proteins and organelles for recognition by the cargo adaptor proteins, phosphorylation regulates the binding affinity of cargo adaptor proteins to the ubiquitinated substrates or autophagosome [10]. Hence, interplay between phosphorylation and ubiquitination modulates the efficiency and specificity of substrate recognition in selective autophagy. Sph1 is also modulated by phosphorylation and ubiquitination [206]. However, the effects of both PTMs on Sph1 have only been studied with regards to Sph1 inclusion formation but not autophagic clearance. Therefore, the first part of this study aims to investigate the potential synergistic roles of both PTMs in modulating different types of Sph1-mediated aggrephagy. We propose that phosphorylation may play an instrumental role in regulating Sph1-mediated basal aggrephagy. This process has been found to be not dependent on K63U [18].

As basal and inducible aggrephagy have distinct molecular mechanisms, we hypothesize that Sph1 engages different interacting partners under physiological and cellular stress condition to perform basal (K63U-independent) and inducible (K63U-dependent) aggrephagy respectively. Hence, we are interested to investigate the potential crosstalk between phosphorylation and $\mathrm{K} 63 \mathrm{U}$ on Sph1, mediated by differential recruitment of protein kinases and E3 ligases, in directing the switch in Sph1 aggrephagy behavior from basal to inducible aggrephagy upon onset of cellular stress. 
In summary, these are the questions we will address for this study:

1. The role of phosphorylation and protein kinases in influencing Sph1-mediated aggrephagy.

2. The possibility of crosstalk between phosphorylation and ubiquitination to potentiate the switch between basal and inducible Sph1-mediated aggrephagy. 


\subsection{MITOCHONDRIAL QUALITY CONTROL AND HOMEOSTASIS}

The human brain consumes more energy than any other organ, accounting for up to 20 percent of the body's total energy expenditure. A resting cortical neuron consumes approximately 4.7 million ATP molecules per second to power various biological functions [207]. Mitochondria are powerhouses that supply more than $90 \%$ of the cellular ATP to support neuronal survival and function. The organelle is important for cell metabolism and energy production via oxidative phosphorylation along the electron transport chain, fatty acid oxidation and Kreb cycle [208]. In addition, mitochondria are responsible for regulating intracellular $\mathrm{Ca}^{2+}$ homeostasis and apoptosis via sequestration of $\mathrm{Ca}^{2+}$ and pro-apoptotic proteins respectively [209]. In the neuronal cells, mitochondria are synthesized at the soma and transported along the axons to the synapses. In the presynaptic terminals, mitochondria produce ATP and buffer $\mathrm{Ca}^{2+}$ levels to support neurotransmission [210]. Aberrant mitochondrial function and transport in neurons has been detected in aging and neurodegeneration [211].

Besides serving critical metabolic functions, mitochondria can also potentially harm cells. The oxidative phosphorylation event generates reactive oxygen species (ROS) including superoxide anions, hydrogen peroxide and hydroxyl radicals as by-products. These ROS create oxidative stress and damage intracellular constituents including proteins and organelles. Furthermore, the situation can be aggravated in the presence of extracellular stressors, where compromised mitochondrial function leads to even more ROS production [212]. Damaged mitochondria could also release $\mathrm{Ca}^{2+}$ and cytochrome $\mathrm{C}$ into the intracellular environment and trigger cell death [213]. Hence, proper mitochondrial activity is important for cell survival and damaged mitochondria must be efficiently removed to prevent cellular damage. A coordinated action between mitochondrial biogenesis and clearance is important for regulating mitochondria health. An imbalance between both processes will lead to accumulation of damaged mitochondria, and has been implicated in development of age-related diseases like cardiomyopathies, psychiatric disorders and neurodegeneration [214]. 


\subsubsection{Mitochondrial biogenesis}

Mitochondria proliferation is a tightly regulated process controlled by expression of mitochondrial and nuclear genes, with the latter accounting for synthesis of more than $90 \%$ of the mitochondrial proteome [215]. Hence, in addition to transcription and translation of mitochondrial proteins, efficient import of nuclear-coded proteins to the respective mitochondria sub-compartments is also important for generating a functional mitochondrion [216-218]. The regulation of mitochondrial biogenesis is achieved by the activation of a few transcription factors in response to diverse stimuli, including nutrient level changes. These transcription factors include nuclear respiratory factor 1 and 2 (NRF1 and NRF2), and peroxisome proliferator-activated receptor gamma co-activator-1 alpha (PGC-1 $\alpha)[219,220]$.

NRF1 and 2 govern the expressions of multiple mitochondrial proteins, including components of the mitochondrial respiratory complex like cytochrome $\mathrm{c}$ oxidase subunit IV (COXIV), components of the mitochondrial import machinery like translocase of the outer membrane mediated protein 20 (TOMM20), components of the glutathione and heme biosynthesis pathways, and finally mitochondrial ribosomal proteins and transfer RNAs (tRNAs). In addition, NRF1 and 2 have been shown to regulate transcription of transcription factor A and B (TFAM and TFB2M) which are important for mitochondrial DNA transcription [221-223]. A more complex regulation of mitochondrial biogenesis is seen with PGC-1 $\alpha$. The transcription factor can interact with NRF1 to regulate the latter's activity [220]. Interestingly, the AMPK signaling pathway shown to upregulate mitochondrial biogenesis in response to starvation or elevated AMP:ATP ratio regulates PGC-1 $\alpha$ activation. AMPK phosphorylates PGC-1 $\alpha$ and induce mitochondrial biogenesis [224]. The activity of PGC-1 $\alpha$ can also be regulated via deacetylation by Sirtuin 1 (SIRT1) [225], as well as $\mathrm{Ca}^{2+} /$ calmodulin-dependent kinase in response to changes in intracellular $\mathrm{Ca}^{2+}$ levels [226, 227]. Hence, PGC-1 $\alpha$ activation is tightly controlled. In mice models of mitochondrial diseases, as well as human models of COXIII and IV deficiencies, overexpression of PGC-1 $\alpha$ have been shown to alleviate mitochondrial dysfunction and increase mitochondrial proliferation and respiratory activity [228]. 
Interestingly, PGC-1 $\alpha$ also regulates autophagic clearance of mitochondria via induction of TFEB expression [113]. Removal of dysfunctional mitochondria is important as they are sources of ROS which damage intracellular constituents like proteins and organelles. Like the removal of protein aggregates, the cell has a series of mitochondrial quality control mechanisms in place to ensure proper folding of mitochondrial proteins, elimination of the damaged mitochondrial proteins, or the entire organelle by mitophagy.

\subsubsection{Mitochondrial quality control mechanisms}

The mitochondria contain its own proteolytic system to degrade misfolded or unfolded proteins in the mitochondrial compartments. Two ATP-dependent AAA protease complexes located at the inner mitochondrial membrane recognize non-native polypeptides and degrade them [229]. In addition, the UPS has been shown to target the outer mitochondrial membrane (OMM) proteins for degradation by several mechanisms. First, the AAA ATPase cdc48/p97/VCP can extract integral membrane proteins and chaperone them for degradation by the proteasome. Second, the OMM proteins mitofusins can be ubiquitinated by Parkin and targeted for UPS removal [230]. Another interesting degradation mechanism exists for targeting the OMM proteins for removal. In this pathway, vesicles containing OMM proteins bud out from the mitochondrial structure via fission, and are delivered to the lysosomes for degradation [231]. The UPS and mitochondrial-derived vesicles allow selective removal of damaged mitochondrial proteins while leaving the whole organelle intact.

The last mitochondrial quality control mechanism, and also the most well-studied, is the mitophagy pathway. In this process, the entire mitochondrion is sequestered into the autophagosome and targeted to the lysosome for degradation (Fig. 1.3). It has been reported that regulation of mitochondrial dynamics via fusion and fission is a way to separate the damaged and healthy mitochondria pools prior to mitophagy initiation, to facilitate selective targeting of the damaged ones for degradation [232]. 


\subsubsection{Mitophagy regulation by mitochondrial dynamics}

Mitochondria are dynamic organelles that undergo fusion and fission processes. At physiological conditions, mitochondria mostly appear as tubular or interconnected structures. On the other hand, upon mitochondrial stress, damaged mitochondria can be separated from the remaining healthy mitochondrial pool via fission and fragmentation to facilitate degradation of the former by mitophagy [233]. An imbalance between the fusion and fission processes has been implicated in the pathogenesis of PD [234]. Fibroblasts from PD patients harboring Parkin mutation displayed reduced mitochondrial fragmentation and enhanced tubular network. However, the fused mitochondria exhibited reduced respiratory capacity and ATP production [234]. Parkin itself is an important player in mitophagy (discussed later); hence, loss of the E3 ligase prevented mitochondria turnover. As a result, damaged mitochondria accumulate alongside with the healthy mitochondrial network, compromising the overall health of the mitochondrial pool.

The mitochondrial fusion and fission processes are regulated by members of a family of GTPases: Drp1, mitofusin 1 and 2 and Opa1 [233, 235]. Drp1 is a fission-promoting protein, whereas mitofusins (located at the OMM) and Opa1 (located at the inner membrane) regulate the fusion process. Drp1 mediates fission by forming a multimeric complex around the mitochondria tubule and creates membrane scission [236]. On the other hand, mitofusin mediates mitochondria fusion via dimerization with another mitofusin in the adjacent mitochondria to promote membrane tethering [237]. Although mitofusin 1 and 2 regulate membrane fusion, a study shows that the efficiency of mitofusin 1 in mediating mitochondria fusion is higher than mitofusin 2 [238]. Nonetheless, the latter is still important for mitochondrial fusion as knockout of mitofusin 2 led to appearance of fragmented mitochondria [237]. Together with mitofusion 1, Opa1 also regulates mitochondrial fusion [239, 240].

Mitochondrial stress like membrane depolarization, which has been linked to mitophagy induction, has also been reported to induce loss of Opa1 and mitofusins [241]. For the latter, the loss of mitofusins is attributed to ubiquitination of the proteins by Parkin which 
will target the mitofusins for proteasomal degradation. As a result of the loss of fusionpromoting factors, fragmentation of mitochondria occurs which separates the damaged mitochondria from the healthy pool to facilitate selective targeting of the former for degradation by mitophagy [242].

Like aggrephagy, selective degradation of mitochondria is facilitated by cargo receptor proteins, which link ubiquitinated damaged mitochondria to the autophagosome. However, non-canonical mitophagy pathways have also been described that are independent of cargo adaptor proteins and ubiquitination.

\subsubsection{Canonical mitophagy: PINK1-Parkin mediated mitophagy}

The most well-studied mitophagy pathway is mediated by PINK1 and Parkin (Fig. 3a). Mutations of these two genes have been implicated in several forms of autosomal recessive $\mathrm{PD}$, highlighting that loss of mitochondrial quality control is one of the pathophysiology of PD [243, 244]. Under physiological conditions, PINK1 is translocated to the mitochondrial matrix, where it is kept inactive via cleavage by several mitochondrial proteases, including mitochondrial processing peptidase (MPP) and presenilinassociated rhomboid-like (PARL) protease. The cleaved PINK1 fragments are subsequently released in the cytosol, where they can be degraded by the proteasome or act as an inhibitor to prevent translocation of Parkin to the mitochondria [245-247].

Under mitochondrial stress that induces loss of mitochondrial membrane depolarization, PINK1 is stabilized on the outer mitochondrial membrane where it associates with the TOM complex [248]. Subsequently, PINK1 undergoes dimerization and is autophosphorylated at T257, S228 and S402. The phosphorylation signals activate PINK1 to promote recruitment of Parkin to the mitochondrial outer membrane and subsequent activation of Parkin [249]. 
The PINK1-mediated activation of Parkin is orchestrated by interplay between phosphorylation and ubiquitination. Parkin contains an N-terminal ubiquitin-like (UBL) domain followed by a new zinc-binding domain called RING0 and the conserved RING1, IBR and RING2 domains [250]. Under physiological conditions, Parkin adopts a closed confirmation and is kept inactive due to the association between the UBL and the RING1 domain. Mitochondrial stress induced by mitochondrial membrane depolarization causes PINK1 to phosphorylate Parkin at 665 which perturbs the association between the UBL and RING1 [251]. Parkin consequently undergoes a conformational change, relieving the auto-inhibition to prime the E3 ligase for full activation [252]. A second phosphorylation event, which involves phosphorylation of the ubiquitin molecule also at S65 by PINK1, is an important upstream event for full activation of Parkin. The phosphorylated ubiquitin binds to the UBL domain of Parkin, which will fully activate Parkin's E3 ligase activity [253]. With this, Parkin will ubiquitinate OMM proteins, which in turn act as recognition tags for recruitment of cargo adaptor proteins for selective targeting of mitochondria to the autophagosome. PINK1 also phosphorylates mitofusin 2 which, in turn, acts as a receptor for the recruitment of Parkin to OMM [254].

Parkin catalyzes formation of $\mathrm{K} 63 \mathrm{U}$ and $\mathrm{K} 27 \mathrm{U}$ on OMM proteins, which serve as ubiquitin tags for recognition by cargo adaptor proteins [255]. p62, optineurin and NBR1 are the known mitophagy cargo adaptors that promote mitophagy [256]. In addition to facilitating linking of ubiquitinated mitochondria to the autophagosome, p62 has been shown to promote formation of mitochondrial aggregates (mito-aggregates) similar to the protein inclusion Agm. However, the requirement of mito-aggregates prior to initiation of mitophagy clearance remains debatable, as mitophagy has been shown to occur in the absence of p62-mediated clustering [257]. In addition, the absolute relevance of cargo adaptor proteins for selectivity in PINK1-Parkin mediated mitophagy is also debatable. Recent studies show that PINK1-Parkin complex can directly interact with the class III PI3-kinase complex via the recruitment of Ambra1, to directly initiate autophagosome membrane biogenesis around the damaged mitochondria [258, 259]. 
Although the PINK1-Parkin mediated mitophagy has been widely reported in-vivo, it remains controversial whether the phenomenon occurs in matured neurons [260]. It appears that the quality of the primary cultured neurons that can survive to long enough after acute exposure to mitochondrial depolarization agent, is the key to examine PINK1Parkin mediated mitophagy in neurons. One study that managed to establish primary neuronal culture that survived long enough following mitochondrial depolarization observed that PINK1-Parkin mediated mitophagy occurs predominantly in the soma [261]. The compartmentalization of mitophagy event in the soma is facilitated by the enhancement in retrograde (kinesin-mediated) and reduction in anterograde (dyneinmediated) transport of the depolarized mitochondria [261]. Interestingly, the somalocalized mitophagy event also parallels the nature of autophagy compartmentalization in the soma versus the axons- autophagosomes generated locally at the soma or synthesized from distinct neuronal compartments are transported to the soma and confined to the region to facilitate degradation [262]. The parallel movements of the depolarized mitochondria and the autophagosomes to the soma suggest that the neurons evolved an intricate mechanism to facilitate efficient removal of damaged mitochondria. We speculate that retrograde transport could also affect the aggrephagy behavior in neurons, since damaged proteins in the distal processes are similarly delivered to the soma for degradation [263].

It is noteworthy to mention that PINK1-Parkin mediated mitophagy is not the only mitochondrial degradation pathway. This is supported by the observation that PINK1 or Parkin knockout mice appear largely normal [264]. Since then, other mitophagy mechanisms have been characterized [265]. Interestingly, these pathways do not depend on presence of canonical selective autophagy markers like ubiquitination or cargo adaptor proteins.

\subsubsection{OMM proteins-mediated mitophagy}

Mitophagy mediated by OMM proteins are independent of ubiquitination (Fig. 3b). BCL2/adenovirus E1B 19 kDa protein-interacting protein 3 (BNIP3) [266, 267], Nix [268] 
and FUN14 Domain Containing 1 (FUNDC1) are OMM proteins but contain the LIR motif feature of the cargo adaptor proteins. Hence, Bnip3, Nix and FUNDC1 can interact with the LC3 on the autophagosome to promote selective targeting of the mitochondria to the autophagosome. In turn, the LIR motifs can be regulated by phosphorylation to influence the interaction with the autophagosome. Phosphorylation of BNIP3's LIR motif at S17 and S24 serve as signals to enhance interaction with autophagosomes [269]. On the other hand, dephosphorylation at $\mathrm{Y} 18$ and phosphorylation at S17 by ULK1 on FUNDC1 induces interaction with the autophagosome [270, 271]. In this paradigm, phosphorylation of ULK1 by AMPK is important for translocation of the former to the damaged mitochondria [270]. FUNDC1 has also been reported to interact with mitochondrial fission protein Drp1 and mitochondria fusion protein OPA1, which suggests a potential role of the OMM protein in regulating mitochondrial dynamics to facilitate mitophagy [271]. Recently, it has been shown that the OMM proteins could also be regulated by phosphoubiquitin. Ubiquitination of OMM proteins by phospho-ubiquitin facilitate their recognition by optineurin and cargo adaptor nuclear dot protein 52kDa (NDP52), to link the mitochondria to the autophagosome [272].

\subsubsection{Lipid-mediated mitophagy}

Lipid moieties on the OMM can also bind directly to LC3 on the autophagosome independent of the canonical cargo adaptor proteins (Fig. 3c). Cardiolipin, the inner mitochondrial membrane phospholipid in eukaryotic cells, can translocate to the OMM and directly interacts with the LC3 on autophagosome. Several kinases are implicated in the translocation of cardiolipin, including nucleoside diphosphate kinase-D, mitochondrial creatine kinase and phospholipid scramblase 3 [273]. Ceramide, a sphingolipid, has also been shown to interact with LC3. Overexpression of ceramide synthase or exogenous administration of ceramide in various cell-based cancer models have been shown to induce mitophagy [274]. 


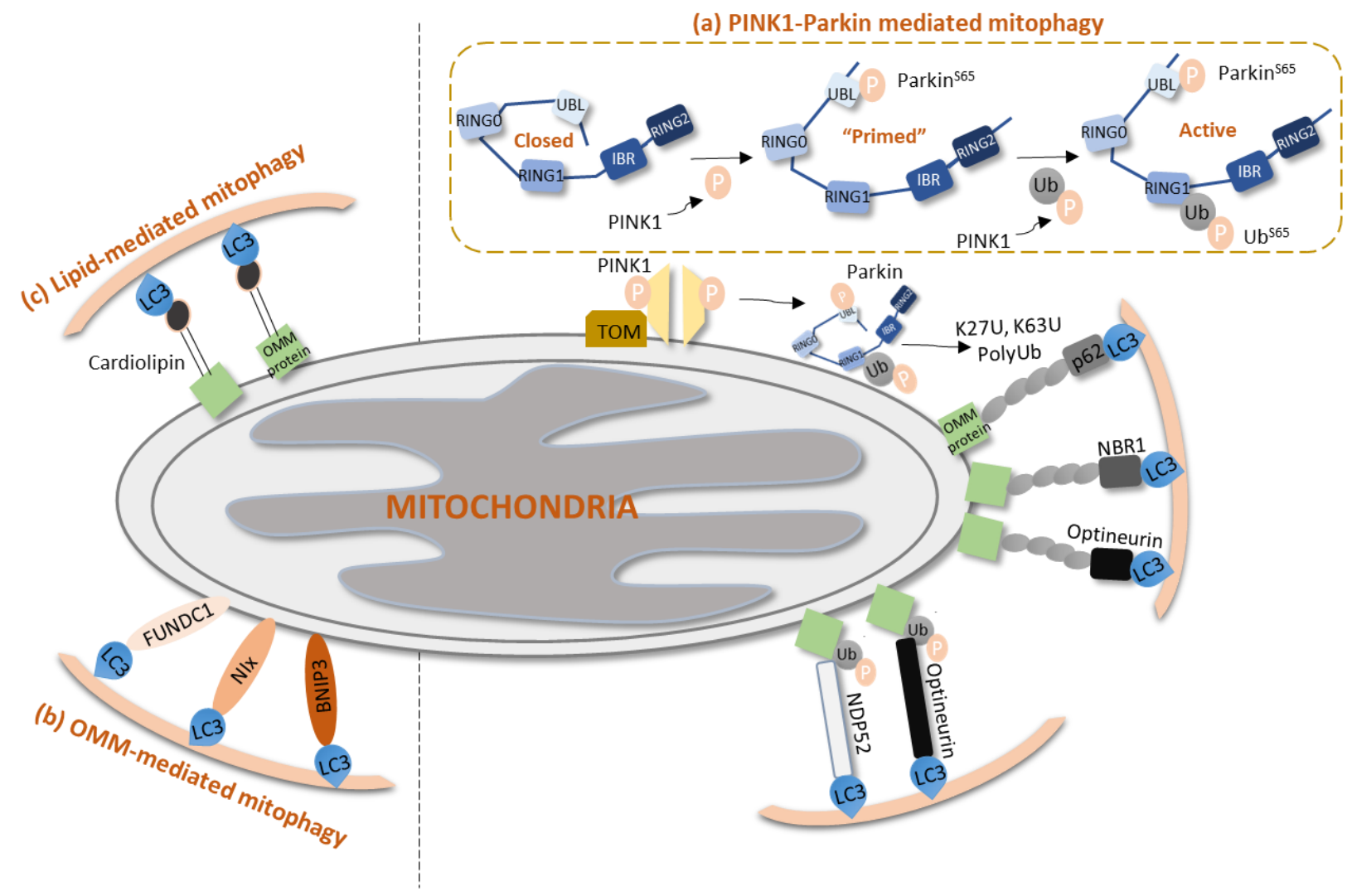

Figure 1.3 The mitophagy pathways. (a) The most well-studied mitophagy pathway is mediated by PINK1 and Parkin. Under mitochondrial stress, PINK1 is stabilized on the outer mitochondrial membrane where it associates with the TOM complex. Subsequently, PINK1 undergoes dimerization and autophosphorylation, and promotes activation and recruitment of Parkin to the OMM. Under physiological conditions, Parkin adopts a closed confirmation and is kept inactive due to the association between the UBL and the RING1 domain. Mitochondrial stress induced by mitochondrial membrane depolarization causes PINK1 to phosphorylate Parkin at S65 which perturbs the association between the UBL and RING1. A second phosphorylation event, which involves phosphorylation of the ubiquitin molecule also at S65 by PINK1, is important for full activation of Parkin. The phosphorylated ubiquitin binds to the UBL domain of Parkin, which will fully activate Parkin's E3 ligase activity. With this, Parkin will ubiquitinate OMM proteins via K27U or $\mathrm{K} 63 \mathrm{U}$, which in turn act as recognition tags for recruitment of cargo adaptor proteins like p62, NBR1 and optineurin for selective targeting of mitochondria to the autophagosome. In addition to modifying Parkin's behavior, OMM proteins could also bind to 
phosphorylated ubiquitin and promote recruitment of cargo adaptors. (b) BNIP3, Nix, and FUNDC1 are OMM proteins containing the LIR motif. Hence, BNIP3, Nix and FUNDC1 can interact with the LC3 on the autophagosome to promote selective targeting of the mitochondria to the autophagosome. (c) Lipid moieties on the OMM can also bind directly to LC3 on the autophagosome independent of cargo adaptor proteins. The inner mitochondrial membrane phospholipid cardiolipin can translocate to the OMM and interact directly with LC3 on the autophagosome. 


\subsubsection{Coordination between mitochondrial biogenesis and mitophagy maintains mitochondrial homeostasis}

The balance between the opposing processes of mitochondria biogenesis and clearance is important for regulating the mass as well as the health of mitochondria. Imbalance between both processes has been implicated in aging and several pathological conditions, where accumulation of dysfunctional mitochondrial mass is observed [211, 232, 275]. Interestingly, mitochondrial biogenesis and degradation share common signaling pathways, which allow tight communication between both processes to regulate mitochondrial homeostasis and cell survival [214].

AMPK. Upregulation of AMPK signaling is associated with activation of PGC-1a, the transcription factor involved in mitochondrial protein synthesis [276]. AMPK could potentially enhance SIRT1 activity which, in turn, deacetylates PGC-1a to activate the transcription factor [225, 277]. AMPK can thus, mediate autophagy and therefore, mitochondrial clearance as well as mitochondrial proliferation simultaneously.

Parkin. In addition to mediating ubiquitination of OMM proteins to facilitate their removal by the proteasome and autophagy, Parkin can interact with TFAM and induce mitochondrial DNA transcription [278]. Parkin has also been demonstrated to target PARIS, a zinc finger transcription factor that negatively regulates PGC-1 $\alpha$ and NRF1 transcriptions, for degradation by the proteasome [279]. Parkin thus promotes mitochondrial homeostasis by enhancing transcription of mitochondrial genes and eliminating damaged mitochondria.

TFEB and PGC-1a. PGC-1a, a transcription factor that regulates mitochondrial biogenesis, also regulates the transcription of TFEB. In turn, TFEB also regulates the transcription of PGC-1 $\alpha$. This process creates a positive feedback loop to maintain mitochondria biogenesis and degradation [113]. 


\subsection{IMPAIRMENTS IN AGGREPHAGY AND MITOPHAGY IN NEURODEGENERATION}

Thus far, we have discussed the mechanisms underlying selective targeting of protein inclusions and damaged mitochondria clearance, including the cargo adaptor dependent and independent selective autophagy pathways. The specification of pathogenic protein inclusions and damaged mitochondria for degradation is important to allow the cell to respond to specific stressors, like proteotoxicity and mitochondrial stress, to remove undesirable substrates while leaving the remaining cellular proteome and healthy organelles untouched.

The importance of aggrephagy and mitophagy in cellular integrity and survival is highlighted under pathological conditions, where accumulation of protein inclusions and damaged mitochondria are observed due to perturbed turnover. Accumulation of protein inclusions is associated with development of proteinopathies. In the liver, buildup of pathogenic aggregates to form hyaline and Mallory-Denk bodies in hepatocytes are observed in hepatocellular carcinoma (liver cancer) and non-alcoholic steatohepatitis (Fatty liver disease) $[280,281]$. Accumulation of toxic protein aggregates in the pancreas to form islet amyloids in $\beta$-cells leads to development of Type 2 Diabetes [282]. On the other hand, mitophagy deficiency has been associated with cancer and heart diseases. Indeed, silencing of Parkin, PINK1 and BNIP3 genes have been implicated in the development of breast cancer carcinoma, hepatocellular carcinoma, adrenal cortical carcinoma and pancreatic cancer tumorigenesis [283]. The initiation and progression of cancers under mitophagy impairment is attributed to accumulation of dysfunctional mitochondria as sources of ROS, which can damage nuclear and mitochondrial DNA and induce mutations and tumorigenesis. Impairment in mitophagy has also been associated with heart diseases [283]. Deficiency of Parkin, PINK1 and mitofusin 2 in mice have been associated with cardiac infarction, early onset of cardiac hypertrophy and dilated cardiomyopathy respectively [283]. 
Of all the different tissue and cell types, aggrephagy and mitophagy impairments cause the more pronounced and deleterious effects in neurons. This is because neurons are post-mitotic cells and cannot rely on cell division as a mechanism to remove protein inclusions and damaged mitochondria [284]. Moreover, the high demand for ATP and mitochondrial activity by neuronal cells makes them more vulnerable to the accumulation of ROS and damaged mitochondria over time. Neuronal cells are therefore more susceptible to mitochondrial stress. The importance of aggrephagy and mitophagy in neurons is underscored in neurodegenerative disorders where impairment in both processes is implicated in PD, HD and amyotrophic lateral sclerosis (ALS).

\subsubsection{Parkinson's disease}

PD is associated with the appearance of intrinsically disordered $\alpha$-synuclein-enriched LB in the substantia nigra of the midbrain [285]. High levels of $\alpha$-synuclein have been shown to prevent recruitment of Atg9 to the phagophore structure via inhibition of Rab1 GTPase. As a result, Atg9 complex-mediated transport of lipids-enriched vesicles to the growing phagophore structure is inhibited, which prevents formation of the autophagosome membrane [286]. In addition, $\alpha$-synuclein has been shown to compromise lysosomal proteolytic capability by decreasing lysosomal acidification [287]. This aggregation-prone protein hence impairs degradation of autophagic substrates by the lysosome. In mitochondria, $\alpha$-synuclein aggregates has been shown to impair mitochondria metabolic activity by inhibiting the mitochondria respiratory complex and inducing mitochondria fragmentation [288].

PINK1 and Parkin mutations are well-known causes for autosomal recessive PD [243, 244]. Parkin's activity is important for facilitating polyubiquitination of protein inclusions and OMM proteins to facilitate recruitment of cargo adaptors. Loss of Parkin (and PINK1 for mitophagy) will thus perturb recognition of the cargos by the autophagic machinery. In addition to PINK1 and Parkin, LRRK2 mutations have been implicated in the development of sporadic PD. The altered LRRK2 activity as a result of the mutations has been 
associated with deregulation of lysosomal $\mathrm{pH}$, mitochondrial fragmentation and enhanced ROS generation [289].

\subsubsection{Huntington's disease}

Polyglutamine (PolyQ) diseases is a group of genetic syndromes associated with abnormal expansion of CAG repeats which affects protein folding and stability [290]. Prominent neurodegenerative conditions associated with polyQ expansion include HD and spinocerebellar ataxia, which mainly affect the cerebellum and striatum respectively [290, 291]. In HD, mutation of the $\mathrm{Htt}$ gene causes synthesis of $\mathrm{Htt}$ proteins with expanded CAG repeats $(>35)$. These mutant $\mathrm{Htt}$ proteins are aggregation-prone and interact with the autophagic machinery and mitochondrial compartment, compromising their activities. For example, mutant $\mathrm{Htt}$ aggregates interact with Beclin-1 and the class III PI3-kinase complex, reducing their availabilities for binding to the phagophore for autophagosome biogenesis [292]. Interestingly, mutant $\mathrm{Htt}$ can also positively regulates autophagy and potentially protects against PolyQ toxicity. Mutant $\mathrm{Htt}$ inclusion bodies have been reported to sequester mTOR, thus attenuating the kinase complex activity to induce autophagy [293].

In mitochondria homeostasis, mutant $\mathrm{Htt}$ interacts with the mitochondria and induces mitochondrial damage via membrane depolarization and alteration of mitochondrial ultrastructure [294]. Mutant Htt also inhibits transcription of PGC-1a [113, 295] and perturbs trafficking of mitochondria in cortical neurons [296]. Hence, when it comes to mitochondrial health and integrity, mutant $\mathrm{Htt}$ deregulates the regeneration and clearance of mitochondria, thus impairing mitochondrial homeostasis.

\subsubsection{Amyotrophic lateral sclerosis}

ALS is characterized by the loss of motor neurons in the brain and spinal cord. Mutations of superoxide dismutase 1 (SOD1) [297] and TAR DNA-binding protein 43 (TDP-43) [298] 
have been implicated in the disease pathogenesis. Mutant SOD1 and TDP-43 aggregates have been shown to interact with OMM proteins like voltage-dependent anion-selective channel 1 (VDAC1) and mitofusin 2, and the former has been shown to accumulate in the mitochondrial matrix and induces oxidative stress [299, 300]. In addition, SOD1 mutant mice display impairments in mitochondrial oxidative phosphorylation activity, alteration to mitochondrial ultrastructure and trafficking [299-301].

Mutations in p62 and optineurin have also been implicated in ALS. Although disruption of the genes has not been directly demonstrated to perturb selective autophagy, their role in recognition of ubiquitinated protein inclusions and damaged mitochondria suggest that the mutations could perturb the recognition of substrates by the autophagic machinery $[180,302]$. 


\subsection{PHARMACOLOGICAL INTERVENTIONS TO MODULATE AUTOPHAGY AS A THERAPY FOR AGING AND NEURODEGENERATION}

Genetic mutations of essential genes in selective autophagy are implicated in the development of neurodegeneration. Gene therapy represents the most ideal therapy for neurodegenerative diseases wherein mutant genes are replaced with the wild-type counterpart [303]. However, gene therapy currently still faces significant safety hurdles which might need many more years of research before becoming a viable therapeutic strategy for neurodegeneration.

Pharmacological intervention with small molecule compounds is the current strategy for neurodegenerative diseases [304]. These compounds serve to upregulate autophagy to enhance clearance of unwanted substrates. Currently, a variety of drugs and compounds have been shown to target different stages of the autophagy pathway and alleviate neurotoxicity in cell and animal neurodegeneration models [305-307]. The first group of compounds modulates the general autophagy pathway by targeting the mTORC1 and AMPK signaling pathways. However, a key caveat for targeting mTORC1 is the off-target effects, given the diverse physiological roles of mTORC1 signaling [308]. Moreover, upregulation of general autophagy does not discriminate between the types of substrates that are being targeted for degradation. A more ideal intervention, hence, is to target the selective autophagy pathway to increase the clearance efficiency of specific substrates while preserving the remaining cellular components.

\subsubsection{Enhancing systemic autophagy activity: Targeting mTOR and AMPK signaling pathways}

Enhancing autophagy activity by modulating the mTORC1 and AMPK signaling pathways are the most common interventions applied to alleviate intracellular burden and toxicity from protein inclusions. Although the modulations target and influence the general, nonselective autophagy pathway rather than the selective clearance of protein inclusions, 
modulating the signaling pathways has shown to be beneficial to fight against neurodegeneration.

Upregulation of autophagy can be first achieved by inhibiting the mTOR pathway. Rapamycin, a lipophilic macrolide antibiotic that binds to mTORC1 and inactivates its activity [95], has been shown to reduce the levels of intracytoplasmic protein inclusions and relieve aggregation-induced toxicity in AD, HD, PD, frontal temporal dementia (FTD), spinocerebellar ataxia (SCA) type III and prion disease models [309-314]. However, given the varied physiological functions of mTOR signaling which also encompasses roles in ribosome biogenesis and cellular metabolism, a key caveat is the possibility of off-target effects [315]. Identification of mTORC1 inhibitors with fewer side effects is thus favorable.

Targeting AMPK signaling, is an alternative to mTOR-dependent signaling pathways to modulate autophagy, and may be a more ideal cellular route. Metformin, an antidiabetic medication, has been shown to be neuroprotective against HD and taupathies by activating AMPK signaling and autophagy [316-318]. In an AD cell model, metformin increases protein phosphatase $2 \mathrm{~A}$ levels and reduces tau phosphorylation and aggregation [317, 318]. Another AMPK activator, 5-amino-4-carboxamide-1- $\beta$-Dribonucleoside (AICAR), also helps to reduce the levels of phosphorylated tau in neuronal cell lines under stress-induced conditions, and may be a potential compound to fight against taupathies [319].

Targeting mTOR-dependent and independent pathways synergistically have also been shown to have enhanced benefits for relieving the burden of intracellular protein aggregates. Administration of rapamycin (mTORC1-dependent) along with lithium (mTORC1-independent), was shown to provide greater protection against neurodegeneration in HD cell and drosophila models than the individual application of the respective compounds [320]. 


\subsubsection{Enhancing selective autophagy: Aggrephagy and mitophagy}

Cargo adaptor proteins and HDAC6 are the most appealing therapeutic targets to modulate aggrephagy, as they are key components that influence selectivity in this process. However, pharmacological compounds that can upregulate the actions of cargo adaptors and HDAC6 are limited. Those attempting to influence their actions focus on altering the expression level and PTM.

Deprenyl, a candidate drug for PD, induces nuclear accumulation and activation of transcription factor Nrf2, which upregulates expression of p62 [321]. This can potentially increase the pool of cargo adaptor proteins available to match with the enhanced levels of protein aggregation during proteinopathies for more efficient autophagic targeting. A number of HDAC6 inhibitors have been demonstrated to be neuroprotective by inhibiting the deacetylase activity of HDAC6 [322, 323]. Trichostatin A leads to enhancement of $\alpha-$ tubulin and promotes effective recruitment of dynein-dynactin and kinesin motor proteins onto microtubules in the HD brain [324]. This drug, along with mercaptoacetamides-based HDAC6 inhibitors, has also been shown to protect against oxidative stress-induced cortical neurons injury [325]. Tubacin, another HDAC6 inhibitor, reduces phosphorylation of tau and could potentially contribute to reduction in levels of hyperphosphorylated tau proteins in taupathies [326]. Inhibitors of other HDAC classes are also found to protect against AD neuropathology. HDAC1 inhibitors like sodium valproate, sodium butyrate and vorinostat are found to reverse cognitive deficits in AD mice [327].

Despite the promising effects of HDAC6 inhibitors in treating neuropathological conditions, caution must be exercised when attempting to modulate HDAC6 deacetylase activity as a solution for protein aggregation-associated diseases [323]. This is because HDAC6 plays multiple roles in the selective aggrephagy cascade. Although inhibition of tubulin deacetylation by HDAC6 can stabilize the microtubule network important for axonal transport or compromise Agm formation and clearance. 
Several compounds, including carbonyl cyanide 3-chlorophenylhydrazone (CCCP), antimycin and oligomycin, have been shown to induce PINK1-Parkin mediated mitophagy in-vitro. However, these compounds stimulate mitophagy by inducing mitochondrial damage, and are unsuitable as therapeutic agents [328]. Sulphoraphane, a natural occurring compound found in cruciferous vegetables, has been shown to improve mitochondrial respiratory activity and bioenergetics in seizure-linked mice models. Furthermore, sulphoraphane has been reported to increase localization of the cargo adaptor protein p62 to the mitochondria and enhance mitophagy. Sulphoraphane is hence a promising therapeutic agent for neurodegeneration [329]. In PD, where PINK1-Parkinmediated degradation of damaged mitochondria is impaired, agents that could upregulate OMM proteins and lipids-mediated mitophagy could potentially be useful for treating this neurodegenerative disorder. 


\subsection{REGULATING AUTOPHAGY VIA DIETARY MODULATION}

Using pharmacological interventions to upregulate autophagy or selective autophagy has shown some success in reducing protein aggregation and alleviating proteotoxicity in mice models [13]. However, the process leading up to the final availability of the drugs for human consumption is a tedious one, encompassing multiple rounds of clinical trials to validate in-vivo efficacy and safety. There is a pressing need thus, to search for safer and more physiological therapeutic options to modulate autophagy to counteract neurogenerative conditions.

\subsubsection{Caloric restriction}

Therapies involving changes to lifestyle practices have recently proven to protect against deleterious protein aggregation and neurodegeneration [16, 17, 330-334]. Caloric restriction (CR), a practice whereby dietary intake is reduced, has been established to prolong lifespan and delay the onset of age-related diseases via autophagy upregulation [330]. The importance of autophagy induction for lifespan expansion has been shown in various autophagy-deficient $C$. elegans and drosophila models, where mutations of Atg7, 12 , and Atg8 reduced the lifespan of the organisms [335, 336]. By constantly maintaining a cellular environment with low nutrient availability, CR can enhance autophagy via activation of the AMPK signaling pathway, or via the SIRT1 deacetylase-forkhead box proteins (FOXO) signaling pathway. Low nutrient supply leads to elevated levels of intracellular nicotinamide adenine dinucleotide (NAD) that activates SIRT1 deacetylation of the FoxO3 transcription factor to enhance transcription of autophagic genes [337, 338]. CR-induced autophagy has been demonstrated to alleviate oxidative stress and accumulation of intracellular waste products, with delayed onset or lower incidences of age-related neurodegenerative pathologies [339-341]. On the other hand, ablation of the autophagy pathway attenuates the beneficial effects of CR [332, 336, 342]. 
However, in humans, long term practice of $\mathrm{CR}$ might not always be a feasible intervention for age-related neurodegeneration or promoting longevity if the lifestyle practice is not managed well by the individuals. Extreme CR can lead to deficiency in essential vitamins and minerals vital for supporting organ functions [343]. Dietary modulation by increasing the intake of polyphenols has instead received much attention as an alternative strategy to promote healthy aging and as a protection against neurodegenerative diseases [16]. This method mimics the autophagy induction observed with CR.

\subsubsection{Polyphenols and the protection against protein aggregates-induced neurotoxicity}

Natural polyphenols are the most commonly found in fruits, vegetables, as well as tea. They were first characterized in plants, where their primary function is to protect the plants from ROS insults [344]. Initially, polyphenols were prominently discussed for their antioxidant effects [345] but the characterization of their ability to induce autophagy has shed light on their potential to alleviate aggregation-induced proteotoxicity observed in neurodegeneration [346]. Here, we will review three polyphenols that have been widely studied for their protective effects against amyloidopathy and protein aggregation.

Resveratrol. Resveratrol is enriched in grape seed and therefore in red wine [347]. In amyloid precursor protein (APP)/presenilin 1 AD transgenic mice, this polyphenol has been shown to activate AMPK signaling to lower the levels of amyloid-beta $(A \beta)$ in the cerebral and cortex, and inhibits mTOR signaling to trigger autophagy and degradation of A $\beta$ peptides [348]. In Tg19959 transgenic AD mice, resveratrol reduces amyloid plaque formation. Resveratrol has also been shown to prolong lifespan through SIRT1 and 2 which induce autophagy [349].

Resveratrol has also been reported to modulate mitochondrial homeostasis by inducing mitochondrial biogenesis via activation of SIRT [350]. Activated SIRT1 mediates deacetylation and downstream activation of PGC-1 1 . Administration of resveratrol to mice 
fed with high fat diet resulted in enhanced mitochondrial mass, along with better motor function and survival of the mice [351].

Epigallocatechin gallate (EGCG). EGCG is enriched in green tea [352], and has been shown to stabilize autophagy via two mechanisms. First, EGCG stabilizes lysosomal proteolytic enzymes and enhance lysosomal degradation of cellular constituents [353, 354]. Second, EGCG could potentially increase the levels of Beclin-1 by inhibiting the antiapoptotic BCL-2-family proteins, $B C L-x(L)$ and $B C L-2$, and facilitate autophagosome biogenesis and autophagy induction [355]. In transgenic HD fly models, EGCG has been shown to inhibit aggregation of mutant $\mathrm{Htt}$ proteins [356]. EGCG promotes nonamyloidogenic processing of APP and prevents toxic $A \beta$ peptides generation in neuronal cell lines overexpressing APP proteins [357]. In 1-methyl-4-phenyl-1,2,3,6tetrahydropyridine (MPTP) oxidative mouse model of PD, EGCG prevents the accumulation of pathogenic $\alpha$-synuclein in the substantia nigra [358].

In mitochondrial homeostasis, the role of EGCG has yet to be reported. But studies have shown that EGCG protects against insults that induce mitochondrial-mediated apoptosis in human lens epithelial cells and dental pulp cells.

Curcumin. Curcumin is derived from turmeric, a type of ginger [359]. In APP/presenilin 1 $A D$ transgenic mice, curcumin reduces the levels of $A \beta$ peptides and formation of amyloid plaques in the brain [360]. In Creutzfeldt-Jakob disease, curcumin has been shown to bind to prion proteins and their intermediates, and potentially reduce the aggregation property of the prion proteins [361]. Curcumin has been shown to have anti-inflammatory and pro-apoptotic properties in addition to autophagy induction [362, 363]. In mitochondrial homeostasis, curcumin has been shown to induce transcription of PGC-1 $\alpha$ in aging mice model and improve ATP levels as well as restoring mitochondria fusion [364]. Curcumin has also been reported to upregulate TFAM expression and induce expression of mitochondrial respiratory chain proteins [364]. 


\subsection{AIM 2: TO INVESTIGATE THE POTENTIAL OF POMEGRANATE AS A FUNCTIONAL FOOD TO MODULATE AGGREPHAGY AND MITOPHAGY}

Overall, while polyphenols have been well-reported to mitigate protein aggregatesinduced toxicity in neurodegeneration [16,346], less is explored about the underlying mechanism for aggrephagy activation by these compounds. More specifically, polyphenols-associated protection against protein aggregation and proteotoxicity in neurodegenerative animal models is primarily related to general autophagy upregulation [16]. No reports so far have shown that polyphenols reduce amyloid protein loads via activation of selective autophagy/aggrephagy. When it comes to mitochondrial homeostasis, literatures often delineate the beneficial roles of polyphenols on mitochondrial biogenesis and functions, but less is explored on their effects on mitochondrial quality control by mitophagy.

Hence, we are interested to investigate if polyphenols are able to influence selective autophagy, by using the pomegranate fruit extract (PE) as the subject of study. Pomegranate is enriched with multiple types of polyphenols, and has been found to alleviate amyloid load in $A D$ by preventing formation and fibrillization of pathogenic $A \beta$ peptide in AD mice model $[365,366]$. In mitochondrial homeostasis, the pomegranate metabolite urolithin $A$ is reported to improve mitochondrial and muscle function in $C$. elegans and mouse models, possibly by potentiating mitochondrial clearance as one of the mechanism to preserve mitochondrial health [367]. However, the key question on whether pomegranate elicits these beneficial properties by modulating selective autophagy remains to be addressed: what is the underlying molecular mechanism for activation of selective autophagy for proteostasis and mitochondrial homeostasis? 
In summary, these are the questions we will address for this study:

1. Effects of PE and its associated polyphenols on the general autophagy-lysosomal pathway, as well as the molecular mechanism for autophagic regulation by PE and its associated polyphenols.

2. The significance for autophagic regulation by $P E$, particularly on selective clearance of protein aggregates (aggrephagy) and damaged mitochondria (mitophagy). 


\section{CHAPTER 2}

MATERIALS AND METHODS 


\subsection{REAGENTS AND ANTIBODIES}

For this section, the reagents, antibodies, cell culture conditions and the general biochemical techniques adopted in the experimental studies are listed first. Following this, the specific assays to investigate autophagy activity, Sph1 phosphorylation status, aggrephagy process, mitochondrial health, and mitophagy were described.

All antibodies and reagents are indicated with their clone number (for antibody when stated), followed by the catalog number and the company where the items were purchased from.

\subsubsection{Plasmids}

Plasmids encoding human kinase isoforms HA-PKCa (Plasmid \#21232), HA-PKCY (Plasmid \#21236), myc-CKIIß (Plasmid \#27088) and HA-GSK3 $\beta$ (Plasmid \#14753) were purchased from Addgene plasmid repository. pEGFP-N1-TFEB (Plasmid \#38119), pMitoTimer (Plasmid \#52659) and mCherry-Parkin (Plasmid \#23956) plasmids were also purchased from Addgene.

HA-WT ubiquitin and HA-K63 ubiquitin mutant were gifts from Kah Leong Lim (Duke-NUS Medical School, Singapore). pBLAST blasticidin-resistant expression plasmid was a gift from Ana Maria Cuervo (Albert Einstein College of Medicine, USA). The GFP-Synphilin1 (Sph1) and mRFP-GFP-LC3 plasmids were gifts from Michael Y. Sherman (Boston University School of Medicine, USA) and Tso-Pang Yao (Duke University of Medicine, USA) respectively. 


\subsubsection{Primary antibodies}

For immunoblotting (IB), the antibodies were diluted in either $1 \%$ or $3 \%$ bovine serum albumin (BSA) (A7030, Sigma) in 1x Tris-buffered saline (TBS) buffer containing 1\% Tween (\#1610781, BioRad) (TBST). The 10x TBS stock was prepared with 50mM Trisbase (H5135, Promega) and $150 \mathrm{mM}$ sodium chloride (433209, Sigma) dissolved in double-distilled water with the final $\mathrm{pH}$ adjusted to 7.4 . To reconstitute $1 \mathrm{x}$ TBS, the $10 \mathrm{x}$ TBS stock was diluted in double-distilled water.

For immunofluorescence (IF), the antibodies were diluted in 1\% IF blocking buffer, reconstituted from $1 \% \mathrm{BSA}$ and $0.06 \%$ fetal calf serum (FBS) (SH30071.03, Thermo Fisher Scientific) dissolved in 1x phosphate-buffered saline (PBS). The 10x PBS stock was prepared from $137 \mathrm{mM}$ sodium chloride, $2.7 \mathrm{mM}$ potassium chloride, $4.3 \mathrm{mM}$ sodium phosphate dibasic and $1.47 \mathrm{mM}$ potassium phosphate dibasic dissolved in double-distilled water with the final $\mathrm{pH}$ adjusted to 7.4. To reconstitute 1x PBS, the 10x PBS stock was diluted in double-distilled water.

The following primary antibodies and their respective dilutions were used for IB and IF studies: AKT (pan) (C67E7) (sc-4691, Cell Signaling Technology (CST)) (IB: 1:1000), $\beta$ actin (ab6276, abcam) (IB: 1:10,000), CKII (6D5) (sc-12739, Santa Cruz) (IB: 1:200, IF: 1:100), c-myc (9E10) (Developmental Studies Hybridoma Bank) (IB: 1:1000), ERK (pan) (clone 16) (610124, BD Bioscience) (IB: 1:1000), GFP (11 814460 001, Roche) (IB: 1:3000), GSK3ß (27C10) (9315, CST) (IB: 1:1000, IF: 1:300), HA (HA-7) (H3663, Sigma) (IB: 1:1000), LAMP1 (H4A3) (Developmental Studies Hybridoma Bank) (IB: 1:1000, IF: 1:300), LC3 (sc-2775, CST for IB, and NB100-2220, Novus for IB and IF) (IB: 1:1000, IF: 1:300), mCherry (ab167453, abcam) (IB: 1:5000), mTOR (sc-2972, CST) (IB: 1:1000), p70/S6 kinase (sc-9202, CST) (IB: 1:1000), PINK1 (ab23707, abcam) (IF: 1:500), phospho-AKT ${ }^{\mathrm{T} 308}$ (sc-9275, CST) (IB: 1:1000), phospho-AKT ${ }^{S 43}$ (sc-9271, CST) (IB: 1:1000), phospho-mTOR ${ }^{\mathrm{S} 2448}$ (sc-2971, CST) (IB: 1:1000), phospho-p44/42 MAPK (ERK1/2) (T202/Y204) (sc-9101, CST) (IB: 1:1000), phospho-p70/S6 kinase ${ }^{T 389}$ (sc-9205, CST) (IB: 1:1000), phospho-TFEB ${ }^{142}$ (ABE1971, Millipore) (IB: 1:3000), phospho- 
ULK1 ${ }^{\text {S555 }}$ (D1H4) (sc-5869, CST) (IB: 1:1000), PKCaßy (H-300) (sc-10800, Santa Cruz) (IB: 1:1000), TOMM20 (ab56783, abcam) (IB: 1:1000, IF: 1:1000), ULK1 (D8H5) (sc8054, CST) (IB: 1:1000).

\subsubsection{Secondary antibodies}

The mouse and rabbit horseradish peroxidase (HRP) conjugated secondary antibodies (3700832 and 3701004 respectively, Sigma) were diluted in 1x TBST, and used at a dilution of 1:10,000 for IB studies. The Alexa 488 (green) and Alexa 555 (Red) Fluorconjugated secondary antibodies (A11034 and A32727 respectively, Invitrogen) were diluted in 1\% IF blocking buffer and used at a dilution of 1:800 for IF studies.

\subsubsection{Inhibitors}

The following chemicals were used to inhibit the various kinase activities: PKC $\alpha, P K C \beta$

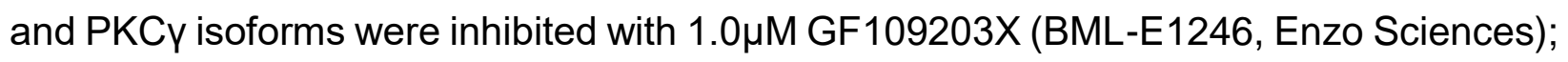

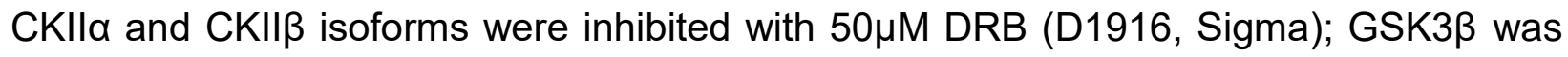
inhibited with 50 $\mu$ M SB415286 (3567, Sigma). Proteasome activity was inhibited with $2 \mu \mathrm{M}$ MG132 (BML-PI102, Enzo Life Sciences).

The following compounds and drugs were used to inhibit the autophagy pathway: the lysosomal activity was inhibited with $20 \mathrm{mM}$ ammonium chloride ( $\mathrm{NH} 4 \mathrm{Cl}$ ) (A9434, Sigma)

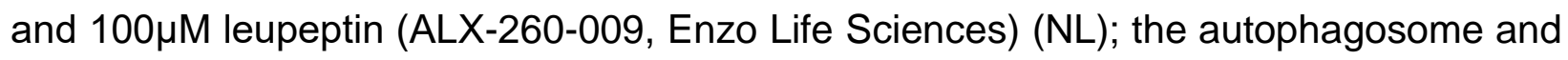
lysosome fusion was inhibited with $1 \mu \mathrm{M}$ (IF) or 10 $\mu \mathrm{M}$ (IB) vinblastine (V1377, Sigma); the autophagosome formation was inhibited with 10mM 3-methyladenine (3-MA) (M9281, Sigma).

The following chemicals were used to inhibit the lysosomal-Ca ${ }^{2+}$ signaling pathway: 5 and

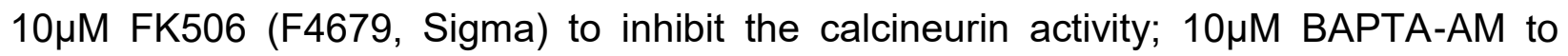


chelate the $\mathrm{Ca}^{2+}$ ions (B1205, Invitrogen). Protein translation and synthesis were inhibited with $10 \mu \mathrm{g} / \mathrm{ml}$ cycloheximide (CHX) (01810, Sigma).

\subsubsection{Mitochondrial depolarization and protein oxidation}

Mitochondrial membrane depolarization was induced by 16 or $24 \mathrm{~h} 10 \mu \mathrm{M} \mathrm{CCCP} \mathrm{(C2759,}$ Sigma) treatment. Protein oxidation was induced by $4 \mathrm{mM} \mathrm{H}_{2} \mathrm{O}_{2}(\mathrm{H} 1009$, Sigma) treatment for $16 \mathrm{~h}$.

\subsubsection{Pomegranate extract (PE)}

$5 \mathrm{mg} / \mathrm{ml}$ PE stock was reconstituted by dissolving a single GNC Herbal Plus $\AA$ Standardized Pomegranate powdered capsule (GNC Singapore) in $5 \mathrm{ml}$ of dimethyl sulfoxide (DMSO) (D2650, Sigma). The HPLC-standardized extract of pomegranate (POMELLA) (Verdure Sciences, USA) [368, 369] was similarly prepared by dissolving $5 \mathrm{mg}$ POMELLA powder in $1 \mathrm{ml}$ DMSO to give a $5 \mathrm{mg} / \mathrm{ml}$ stock. For the PE study, a series of PE and POMELLA concentrations ranging from $50 \mu \mathrm{g} / \mathrm{ml}$ to $1000 \mu \mathrm{g} / \mathrm{ml}$ were tested. The working concentrations for PE or POMELLA were reconstituted by diluting the concentrated stock in culture media.

Bioactive compounds found in pomegranate were purchased from Sigma and reconstituted as indicated: Ellagic acid (EA) was dissolved in DMSO to give $0.1 \mathrm{mg} / \mathrm{ml}$ stock, and 500 and $1000 \mathrm{ng} / \mathrm{ml}$ were used as working concentrations (E2250); Punicalagin (Puni) was dissolved in methanol to give $5 \mathrm{mg} / \mathrm{ml}$ stock, and 50 and $100 \mu \mathrm{M}$ were used as working concentrations (P0023); Delphinidin chloride (DC) was dissolved in DMSO to give $5 \mathrm{mg} / \mathrm{ml}$ stock, and 100 and $200 \mu \mathrm{g} / \mathrm{ml}$ were used as working concentrations (43725); Luteolin (Lut) was dissolved in ethanol to give $5 \mathrm{mg} / \mathrm{ml}$ stock, and 50 and $100 \mu \mathrm{M}$ were used as working concentrations (L9283). 
For all PE or POMELLA-related experiments, DMSO was used as the vehicle control. The volume of DMSO added was equivalent to the largest PE or POMELLA volume used in the experimental set. 


\subsection{CELL CULTURE AND TRANSFECTION}

\subsubsection{Growth conditions}

Human embryonic kidney (HEK) cells, mouse embryonic fibroblast (MEF) cells and SY5Y neuroblastoma cells were purchased from American Type Culture Collection (ATCC, USA). The cells were cultured in Dulbecco's Modified Eagle's Medium (DMEM) (12100046, Invitrogen). Retinal pigmented epithelium (RPE1) cells were a gift from Lei Lu (School of Biological Sciences, NTU, Singapore), and the cells were grown in DMEM/F12 (11320033, Invitrogen) media. All media were supplemented with 10\% FBS and 1\% penicillin-streptomycin (SV30010, Thermo Fisher Scientific) (designated as S+ media). All the cell lines were grown in $5 \% \mathrm{CO} 2$ at $37^{\circ} \mathrm{C}$.

\subsubsection{Transfection and treatments}

Transfection was done in a 6-well plate format for IB experiments, and in a 24-well plate format for IF experiments. In a 6-well plate format for IB experiments, cells were seeded at a confluency of $2 \times 105$ cells per well for transfection. In a 24 -well plate format for IF experiments, cells were seeded at a confluency of $5 \times 104$ cells on coverslip per well for transfection. The cells were seeded and allowed to recover for $24 \mathrm{~h}$ before initiating transfection.

Treatment experiments were similarly conducted in a 6-well plate format for IB experiments, and in a 24-well plate format for IF experiments. In a 6-well plate format for IB experiments, cells were seeded at a confluency of $1 \times 106$ cells per well. In a 24 -well plate format for IF experiments, cells were seeded at a confluency of $2 \times 105$ cells on coverslip per well. The cells were seeded and allowed to recover for $24 \mathrm{~h}$ before initiating treatment. 
For transfection, cells were transfected using the Lipofectamine 2000 reagent (11668019, Invitrogen), with 1:3 DNA to lipofectamine ratio following the manufacturer's protocol. Briefly, the DNA and lipofectamine was separately incubated in serum-free DMEM media media for 5 mins. After this, the lipofectamine mixture was added to the DNA mixture and allowed to incubate for 20mins. The DNA-lipofectamine mix was then added to the cells.

\subsubsection{Generation of mCherry-Parkin, GFP-TFEB and MitoTimer SY5Y stable cells}

SY5Y cells were co-transfected with $1.0 \mu \mathrm{g}$ mCherry-Parkin, GFP-TFEB or MitoTimer plasmids separately with $1.0 \mu \mathrm{g}$ blasticidin-resistant vector for $72 \mathrm{~h}$ in a single 6 -well to generate the respective stable cells. As a vector control, a blasticidin-resistant SY5Y stable cell line was raised by singly transfecting $1.0 \mu \mathrm{g}$ blasticidin-resistant vector for $72 \mathrm{~h}$ in a single 6-well.

At the end of $72 \mathrm{~h}$ transfection, the various transfected cells were plated at 1:100 and $1: 200$ dilutions in the $10 \mathrm{~cm}$ plates for selection of co-transfected cells expressing both the plasmid of interest and the blasticidin-resistant vector, or just the single blasticidinresistant vector transfected cells. The transfected cells were selected by killing the nontransfected cells with $10 \mu \mathrm{g} / \mathrm{ml}$ blasticidin (A1113903, Invitrogen) for $5-7$ days in the $10 \mathrm{~cm}$ plate, until only sparsely spread-out viable single cells remained. Thereafter, the cells that survived the $10 \mu \mathrm{g} / \mathrm{ml}$ blasticidin treatment were allowed to grow into individual cell colonies under $2 \mu \mathrm{g} / \mathrm{ml}$ blasticidin. Positive clones were isolated, and allowed to grow and expand in the presence of $2 \mu \mathrm{g} / \mathrm{ml}$ blasticidin. All stable cell lines created were subsequently maintained in $S+$ media supplemented with $2 \mu \mathrm{g} / \mathrm{ml}$ blasticidin.

\subsubsection{Short interfering RNA (siRNA)-mediated knockdown (KD) in SY5Y cells}

Predesigned siRNA directed against human target genes were used to transiently KD gene expression of TFEB and the kinases. SY5Y cells were transfected with the respective siRNA represented in Table 1 for $48 \mathrm{~h}$, using the lipofectamine method as 
described earlier in section 2.2.2. The company where the various siRNA oligos was purchased from, along with the catalogue number, sequence as well as the concentrations used in the KD experiments were represented in Table 1. As a negative control, cells were transfected with an equivalent concentration of the scrambled siRNA (1027280, Qiagen AllStars negative control; 4390843, Silencer Select Negative Control No. 1 siRNA) likewise for $48 \mathrm{~h}$ using the lipofectamine method.

Table 1: siRNA against TFEB, PKC $\alpha$, PKC $Y$, CKII $\beta$ and GSK3 $\beta$.

\begin{tabular}{|c|c|c|c|}
\hline Gene & $\begin{array}{l}\text { Company of } \\
\text { purchase and } \\
\text { Cat \# }\end{array}$ & siRNA Sequence (5'-3') & $\begin{array}{l}\text { Concentration } \\
(\mathrm{nM})\end{array}$ \\
\hline TFEB & $\begin{array}{c}\text { Qiagen } \\
\text { SI00094969 }\end{array}$ & $\begin{array}{l}\text { Sense: GCCUGGAGAUGACCAACAATT } \\
\text { Antisense: UUGUUGGUCAUCUCCAGGCG }\end{array}$ & 25 \\
\hline PKCa & $\begin{array}{l}\text { Qiagen } \\
\text { siRNA \#1: } \\
\text { SI00605934 } \\
\text { SiRNA \#2: } \\
\text { SI00605927 }\end{array}$ & $\begin{array}{l}\text { siRNA \#1 Sense: CAGUGGAAUGAGUCCUUUATT } \\
\text { siRNA \#1 Antisense: UAAAGGACUCAUUCCACUGCG } \\
\text { siRNA \#2 Sense: CAAGUUGCUUAACCAAGAATT } \\
\text { siRNA \#2 Antisense: UUCUUGGUUAAGCAACUUGTA }\end{array}$ & 50 \\
\hline PKCY & $\begin{array}{l}\text { Qiagen } \\
\text { siRNA \#1: } \\
\text { SI03087763 } \\
\text { SiRNA \#2: } \\
\text { SI03078306 }\end{array}$ & $\begin{array}{l}\text { siRNA \#1 Sense: CGGAGAUCAUUGCCUACCATT } \\
\text { siRNA \#1 Antisense: UGGUAGGCAAUGAUCUCCGGG } \\
\text { siRNA \#2 Sense: ACGCCAUCAAGAUCUUGAATT } \\
\text { siRNA \#2 Antisense: UUCAAGAUCUUGAUGGCGUAG }\end{array}$ & 75 \\
\hline CKII $\beta$ & $\begin{array}{l}\text { Ambion } \\
\text { siRNA \#1: } \\
\text { s3642 } \\
\text { siRNA \#2: } \\
\text { s3643 }\end{array}$ & $\begin{array}{l}\text { siRNA \#1 Sense: GGACGGUUUCAAGAUTT } \\
\text { siRNA \#1 Antisense: AUCUUGAAACGUAGAGCCTG } \\
\text { siRNA \#2 Sense: AGAGUGACCUGAUUGAGCATT } \\
\text { siRNA \#2 Antisense: UGCUCAAUCAGGUCACUCUGG }\end{array}$ & 10 \\
\hline GSK3 $\beta$ & $\begin{array}{l}\text { Ambion } \\
\text { siRNA \#1: } \\
\text { s6239 } \\
\text { siRNA \#2: } \\
\text { s6241 }\end{array}$ & $\begin{array}{l}\text { siRNA \#1 Sense: CGAGAGCUCCAGAUCAUGATT } \\
\text { siRNA \#1 Antisense: UCAUGAUCUGGAGCUCUCGAT } \\
\text { siRNA \#2 Sense: CUCAAGAACUGUCAAGUAATT } \\
\text { siRNA \#2 Antisense: UUACUUGACAGUUCUUGAGTG }\end{array}$ & 5 \\
\hline
\end{tabular}




\subsection{RNA EXTRACTION AND REVERSE TRANSCRIPTION (RT-PCR)}

The efficiency of siRNA-mediated silencing of TFEB was checked by RT-PCR analysis. Post-72h transfection, the scrambled siRNA and TFEB siRNA transfected cells were trypsinized and harvested for RNA extraction using Ambion Purelink RNA mini kit (12183018A, Life Technologies). The extracted RNA was subsequently treated with RQ1 RNase-Free DNase (M6101, Promega) to degrade any DNA contaminants following the manufacturer's protocol. Next, the RNA was concentrated via ethanol precipitation. Briefly, $78 \mu \mathrm{l}$ of sterile water, $10 \mu \mathrm{l}$ of $3 \mathrm{M}$ sodium acetate and $300 \mu \mathrm{l}$ of $70 \%$ ethanol was added to the RNA sample, and centrifuged at $12,500 \mathrm{~g}$ at $4^{\circ} \mathrm{C}$ for $1 \mathrm{~h}$. The supernatant was discarded and the RNA was washed with $70 \%$ ethanol by centrifuging at the same speed at $4^{\circ} \mathrm{C}$ for 30 mins. The RNA was subsequently reverse transcribed using the M-MLV Reverse Transcriptase (M1701, Promega) following the manufacturer's protocol. Briefly, $10 \mathrm{mM}$ of dNTPs and $10 \mathrm{mM}$ of oligoDTs were added to $0.5 \mu \mathrm{g}$ of RNA, and the mixture was topped up with $7.5 \mu \mathrm{l}$ of sterile water. The mixture was then incubated at $65^{\circ} \mathrm{C}$ for $5 \mathrm{mins}$, and chilled on ice after heat incubation. $1 \mu \mathrm{l}$ of the M-MLV reverse transcriptase and $1.5 \mu \mathrm{l}$ of reaction buffer were added the RNA mixture, and the reverse transcription was allowed to proceed by incubating the RNA mixture at $42^{\circ} \mathrm{C}$ for $1 \mathrm{~h}$. The reverse transcription was terminated by inhibiting the reverse transcriptase with heat incubation at $65^{\circ} \mathrm{C}$ for 20 mins. The resulting cDNA product from the reverse transcription was amplified with GoTaq Flexi DNA Polymerase (M8301, Promega) following manufacturer's protocols.

The following primers were used for the PCR amplification: TFEB forward primer 5' GTAGGACTGCACCTTCAACACCT-3', TFEB reverse primer 5'TCACGCATAGGGTTGCGCAT-3'. Actin forward primer 5'CCAGAGGCGTACAGGGATAG-3', Actin reverse primer 5'CCAACCGCGAGAAGATGA-3'.

The following steps and PCR conditions were used for CDNA amplification. Briefly, a PCR reaction mix containing $2.5 \mu \mathrm{l}$ of $5 x$ GoTaq buffer, $0.5 \mu \mathrm{l}$ of $25 \mathrm{mM} \mathrm{MgCl} 2,0.25 \mu \mathrm{l}$ of $10 \mathrm{mM}$ 
dNTPs topped up with $8.125 \mu \mathrm{l}$ of sterile water was prepared. $0.125 \mu \mathrm{l}$ GoTaq polymerase with $0.25 \mu \mathrm{l}$ of the respective TFEB/actin forward and reserve primer were then added. At the final step, $0.5 \mu \mathrm{l}$ cDNA sample was added to the PCR reaction mix. The following stepwise PCR cycling condition was used to amplify the CDNA: Initial denaturation of the DNA at $95^{\circ} \mathrm{C}$ for $5 \mathrm{mins}$, followed by 35 cycles of denaturation $\left(95^{\circ} \mathrm{C}\right.$ for $\left.30 \mathrm{~s}\right)$-annealing $\left(58^{\circ} \mathrm{C}\right.$ for $\left.40 \mathrm{~s}\right)$-extension $\left(72^{\circ} \mathrm{C}\right.$ for $\left.50 \mathrm{~s}\right)$. The final extension step was carried out at $72^{\circ} \mathrm{C}$ for 5 mins.

The KD efficiency of TFEB was examined by running the amplified TFEB DNA sample from the scrambled siRNA and TFEB siRNA transfected cells with a $2 \%$ agarose DNA gel. The reduction in TFEB levels in the TFEB siRNA transfected cells as compared to the scrambled siRNA transfected cells (after normalization to the actin levels) will indicate the KD of TFEB. 


\subsection{CELL LYSIS, SODIUM DODECYL SULFATE POLYACRYLAMIDE GEL ELECTROPHORESIS (SDS-PAGE) AND DENSITOMETRY}

\subsubsection{Cell lysis}

The proteins were extracted from the cells by first scraping the cells in ice cold 1x PBS followed by cell lysis in the respective lysis buffer ${ }^{\#}$ supplemented with protease inhibitor cocktail (prepared from leupeptin hemisulfate (BP266225, Thermo Fisher Scientific) and AEBSF (BP2644500, Thermo Fisher Scientific), Pepstatin A (P-4265, Roche) and PhosSTOP ${ }^{T M}$ phosphatase inhibitor (04906837001, Roche)) on ice with intermittent vortexing.

\#Different types of lysis buffer were used depending on the nature of the proteins to be extracted. To extract general intracellular proteins (total soluble and membrane-bound proteins) in PE study for IB, the radioimmunorecipitation assay (RIPA) buffer (reconstituted from 50mM Tris-base $\mathrm{pH} 7.4$, 1\% nonyl phenoxypolyethoxylethanol -40 (NP-40) (18896, Sigma), 0.5\% sodium deoxycholate (D6750, Sigma), 150mM sodium chloride, 1mM ethylenediaminetetraacetic acid (EDTA) (03620, Sigma) in PBS) was used. For immunoprecipitation (IP) experiment to analyze the ubiquitination profile on Sph1, the cells were lysed under highly denaturing condition with RIPA buffer supplemented with $6 \mathrm{M}$ urea (V3171, Promega). The lysate was subsequently sonicated with $2 \times 5$ s pulses at $20 \%$ amplitude to extract out the total Sph1 proteins (both the soluble and insoluble forms of Sph1). For co-immunoprecipitation (co-IP) experiments in Sph1 study, the cells were lysed under mild detergent condition with 1\%-NP40 to preserve the protein interactions. Finally, to separate the proteins from the cell debris, the lysate was centrifuged at $14,000 \mathrm{~g}$ for $15 \mathrm{mins}$ in $4^{\circ} \mathrm{C}$ condition. 


\subsubsection{SDS-PAGE}

The concentrations of the protein lysate were determined with the BioRad Bradford Protein Assay (5000006, BioRad) with BSA as standards according to manufacturer's protocol. Routinely, $\sim 20-30 \mu \mathrm{g}$ of protein samples were analyzed by SDS-PAGE. The protein lysate was mixed with $2 x$ sample buffer, and separated with the SE 260 Minivertical Unit (SE260-10A-.75, GE Healthcare Life Sciences) for SDS-PAGE. The protein lysates were fractionated at $100-150 \mathrm{~V}$ for $1-1.5 \mathrm{~h}$, and transferred onto the nitrocellulose membrane $(0.45 \mu \mathrm{m}$ pore size) $(1620115$, BioRad) using the wet electrotransfer method for $2 \mathrm{~h}$ at $0.4 \mathrm{~A}$ at $4^{\circ} \mathrm{C}$. The nitrocellulose membrane were subsequently blocked in commercially available $5 \%$ low fat milk prepared in $1 \times$ TBST for $1 \mathrm{~h}$, and probed with the respective primary antibodies with overnight incubation at $4^{\circ} \mathrm{C}$. Post primary antibody binding, the membrane was washed in 1x TBST for $5 \mathrm{mins}$ three times, followed by incubation with the corresponding secondary antibodies for $1 \mathrm{~h}$ at room temperature. The membrane was subsequently washed again with $1 \mathrm{x}$ TBST for 5 mins three times, and the protein bands were visualized via chemiluminescence method using the Pierce ECL Western Blotting Substrate detection kit (32106, Thermo Fisher Scientific) and radioautography $\mathrm{x}$-ray film (Kodak, Singapore)

\subsubsection{Densitometry analysis}

Analysis of the protein band intensities in the immunoblots were quantified using Image J software $(\mathrm{NIH})$. The immunoblots were scanned and converted to an 8-bit image. The background was subtracted using the tabs "Analyze> Subtract Background". Using the "box" tool, the region containing the band was highlighted, and the "Analyze> Measure" tabs were used to measure the mean intensity of the highlighted region. The size of the "box" tool was kept constant throughout the measurement of the intensity of other protein bands in the same gel. Actin or tubulin protein levels were used as loading controls to normalize the band intensity for the protein of interest under the different treatment conditions within the same experiment set. 


\subsection{IMMUNOFLUORESCENCE AND ELECTRON MICROSCOPY}

For IF studies, SY5Y cells on the coverslip was fixed with 4\% paraformaldehyde (PFA) (P6148, Sigma) at $37^{\circ} \mathrm{C}$ for 30 mins, followed by permeabilization with $0.1 \%$ Triton $\mathrm{X}-100$ (161-0407, BioRad) at room temperature for 5 mins. The cells were then stained with the respective primary and secondary antibodies with the concentrations and conditions described in sections 2.1.2 and 2.1.3. The coverslip was mounted onto glass slide with DAPI-containing prolonged gold antifade mountant (P36931, Invitrogen). The images were acquired with Zeiss Z1 AxioObserver inverted fluorescence microscope using the $63 x$ oil immersion objective lens. Random fields on the coverslip were acquired and the exposure time for each wavelength channel was kept constant for all images in the same set of experiment. For data analysis, unedited images were used for Image $\mathrm{J}$ quantification (unless otherwise stated). For presentation in this thesis, the images were presented as "best-fit" images edited using the "best-fit" function in the Zen software (Zeiss) to improve the quality (brightness and contrast) for presentation/publication purpose.

For electron microscopy, SY5Y cells treated with DMSO or $150 \mu \mathrm{g} / \mathrm{ml}$ PE for $24 \mathrm{~h}$ were fixed in $2.5 \%$ glutaraldehyde solution, embedded and sectioned for transmission microscopy imaging at Electron Microscopy Unit facility at NUS, Singapore. 


\subsection{TRYPAN BLUE CELL VIABILITY ASSAY}

Trypan blue is a stain used to selectively color dead cells blue. Live cells with intact cell membranes are not colored, as the trypan blue cannot pass through the membrane and be absorbed. Hence, live cells remain transparent when observed under the light microscope.

The protective effects of PE against oxidative stress was examined by measuring the cell viability of SY5Y cells under $4 \mathrm{mM} \mathrm{H} 2 \mathrm{O} 2$-induced oxidative stress. SY5Y cells were treated with DMSO, 150 or $300 \mu \mathrm{g} / \mathrm{ml} \mathrm{PE}$ under basal condition or in the presence of $4 \mathrm{mM}$ $\mathrm{H} 2 \mathrm{O} 2$ for $16 \mathrm{~h}$ in a 6-well plate format. Post-treatment, the cells were trypsinized and a

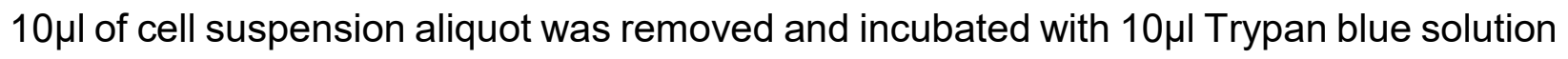
(T8154, Sigma) for 5mins at room temperature. Live (transparent) and dead (blue) cells were counted using haemocytometer with an Olympus CKX21 light microscope using the $10 x$ objective lens. A minimum of 300 cells were quantified, and the cell viability index (or percentage of viable cells) under each treatment condition was quantified by dividing the number of transparent cells over the total cell count multiplied by 100 . 


\subsection{ASSAYS TO MONITOR AUTOPHAGY ACTIVITY}

\subsubsection{LC3-II flux}

LC3-II flux was measured by examining the magnitude of LC3-II accumulation under autophagic inhibition. Higher accumulation of LC3-II will indicate a higher rate of autophagosome turnover by the lysosomes. In our study, we used NL to neutralize the lysosomal acidic $\mathrm{pH}$ and protease activities respectively to inhibit the lysosomes.

PKC, CKII and GSK3 $\beta$ have been implicated in the regulation of autophagy [370, 371]. The effect of kinase inhibitions on the general autophagy was investigated to examine whether PKC, CKII and GSK3 $\beta$ isoforms modulate Sph1-mediated aggrephagy directly. For this analysis, LC3-II flux in SY5Y cells under proteasomal inhibition untreated or cotreated with the various kinase inhibitors were analyzed. Cells were either left untreated,

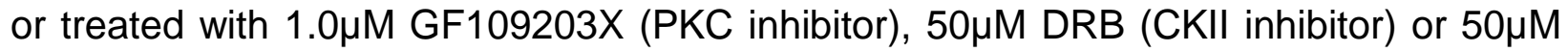
SB415286 (GSK3 $\beta$ inhibitor) in the presence of $2 \mu \mathrm{M}$ MG132 proteasomal inhibition for 16h. Thereafter, the cells were recovered in fresh $\mathrm{S}+$ media with and without lysosomal inhibitors NL for $4 \mathrm{~h}$.

The protective effects of pomegranate and its associated polyphenols are partially overlapped with autophagy induction [372, 373]. The ability of PE or POMELLA to upregulate autophagy in SY5Y cells examined by measuring the LC3-II flux. SY5Y cells were treated with DMSO, or with increasing PE concentrations $(50-300 \mu \mathrm{g} / \mathrm{ml})$, or with $300 \mu \mathrm{g} / \mathrm{ml}$ POMELLA for $24 \mathrm{~h}$. Following this, the media was replaced with either $\mathrm{S}+$ media, or $\mathrm{S}+$ containing lysosomal inhibitors $\mathrm{NL}$ for $4 \mathrm{~h}$.

Post-treatments, the cells were lysed with RIPA buffer and the proteins were separated with SDS-PAGE as described in sections 2.4.1 and 2.4.2. The membranes were immunoblotted for LC3, and the LC3-II flux was measured by quantifying the LC3-II level 
under lysosomal inhibition over the untreated level using the Image $\mathrm{J}$ method as described in section 2.4.3.

\subsubsection{GFP-mRFP-LC3 autophagic flux}

As a complementary method to LC3-II flux to assay autophagic activity under PE treatment, the tandem GFP-mRFP-LC3 was used to analyze the levels of autophagosome and autophagolysosome compartments as a readout for the autophagy flux. The assay is based on the different $\mathrm{pH}$ stability of the GFP and RFP fluorescent proteins, where the former loses it fluorescence signal due to the lysosomal acidity. Hence, the tandem GFP-mRFP-LC3 can be used to label autophagosomes before and after fusion with the lysosomes [374]. LC3 puncta harboring the GFP signal will represent the autophagosomes. After fusion with the lysosomes, the GFP signal will be attenuated and the RFP-labelled LC3 puncta will indicate the autophagolysosomes [374].

SY5Y cells were seeded on coverslips in a 24-well plate, and transfected with 0.5 ug GFPmRFP-LC3 for $24 \mathrm{~h}$ using the lipofectamine method. The cells were then treated with either DMSO, or 150 or $300 \mu \mathrm{g} / \mathrm{ml} \mathrm{PE}$ for $16 \mathrm{~h}$. After the treatment, the coverslips were mounted and viewed as described in section 2.5. The numbers of green and red puncta per transfected cell were quantified. The puncta counts were normalized against the cell area and plotted.

\subsubsection{Immunofluorescence analysis of the number and size of autophagosomes and lysosomes}

To complement the immunoblotting method to evaluate the autophagy activity upon PE treatment. the changes in the autophagosome and lysosome levels in SY5Y cells were monitored with IF. SY5Y cells were seeded onto coverslips in the 24-well plate format, and treated with $300 \mu \mathrm{g} / \mathrm{ml}$ of PE for $24 \mathrm{~h}$. To label the autophagosomes and lysosomes respectively, the cells were stained for anti-LC3 or LAMP1 respectively. The average LC3 
or LAMP1 puncta per cell area and puncta size were quantified under the different treatment conditions as a readout for autophagy activity in the cells.

The numbers of LC3 and LAMP1 puncta, as well as the average size of the puncta were determined using Image J. The overlay image was separated into its respective fluorescence channels which will convert each channel into 8-bit (grayscale) display. The scale was then set to measurement unit " $\mu \mathrm{m}$ ". The outline of the cell was highlighted using the polygon selection tool and the area was measured with the "Analyze>Measurement" tabs. With the outline of the cell still highlighted, the region of interest was selected and the LC3 or LAMP1 puncta were highlighted by manually thresholding the minimal and maximal pixel values using the "Image> Adjust> Threshold" tabs. Consistent threshold values were used across all treatment conditions in the same experimental set to highlight the puncta. Once the threshold pixels were determined, the number of LC3 or LAMP1 puncta, and the average size of the puncta were determined with the "Analyze> Analyze particle" tabs with the size requirement standardized at "0-infinity" pixel and the circularity at "0-1". For the final quantification, the number of LC3 or LAMP1 puncta tabulated per cell was normalized against the cell area. 


\subsection{TFEB NUCLEAR LOCALIZATION}

TFEB is a transcription factor that binds to the promoters of autophagy-related genes and upregulate the expression of autophagic proteins during starvation [104]. Activation of TFEB is associated with enhanced nuclear translocation of the transcription factor [91, 119]. The possibility of PE and its associated polyphenols in regulating TFEB activation and hence autophagy upregulation was investigated by examining the effect these compounds on TFEB nuclear localization. GFP-TFEB stable cells were treated with $300 \mu \mathrm{g} / \mathrm{ml} \mathrm{PE}$ for $2-24 \mathrm{~h}$, or the respective concentrations of the phenolic compounds stated in section 2.1.6 for $16 \mathrm{~h}$. The percentage of cells containing TFEB nuclear localization was quantified under each condition by dividing the number of cells containing nuclear TFEB over the total number of transfected cells quantified multiply by 100 . 


\subsection{ASSAYS TO INVESTIGATE PHOSPHORYLATION STATUS OF SYNPHILIN-1}

\subsubsection{Mass spectrometry}

To determine if Sph1 is phosphorylated, SY5Y cells were transfected with 2.0 $\mathrm{ug}$ GFPSph1 for 48h. Post-transfection, cells from a total of 206 -well plates were pooled together and lysed in $18 \mathrm{ml}$ of RIPA buffer with $6 \mathrm{M}$ urea to extract the total Sph1 (both soluble and insoluble) protein pool. $1.8 \mathrm{ml}$ of GFP-TRAP agarose beads (gta-100, Chromotek) were allowed to incubate with the protein lysate with continuous mixing for $2 \mathrm{~h}$ at $4^{\circ} \mathrm{C}$. GFPSph1 were then eluted from the GFAP-TRAP agarose beads by adding $50 \mu \mathrm{l} 0.2 \mathrm{M}$ glycine $\mathrm{pH} 2.5$ (under constant mixing), followed by centrifugation at $2500 \mathrm{~g}$ for 2 mins. The eluted immunopurified GFP-Sph1 lysate was transferred to a new Eppendorf tube and $5 \mu 1 \mathrm{M}$ Tris base $\mathrm{pH} 10.4$ was added for neutralization.

The immunopurified Sph1 lysate was mixed with 2x sample buffer and separated in an $8 \%$ SDS-PAGE gel as described in section 2.4.2. The gel was subsequently stained with Coomassie blue (prepared by dissolving 1g Coomassie Brilliant Blue R250 (20278, Thermo Fisher Scientific) in $300 \mathrm{ml}$ methanol, followed by additions of $650 \mathrm{ml}$ of distilled water and $50 \mathrm{ml}$ of acetic acid to give a final volume of $900 \mathrm{ml}$ Coomassie blue staining solution) to visualize the purified Sph1 protein band. The gel was destained by incubating with $1 \%$ acetic acid overnight with constant mixing. After destaining, the Sph1 band was excised and approximately $2 \mathrm{mg}$ of proteins (estimated by BradFord assay described in section 2.4.2) was sent for liquid chromatography-mass spectrometry (LC-MS/MS) analysis to identify the putative phosphorylation sites on Sph1. The sample preparation (after obtaining the excised Sph1 band), performance of LC-MS/MS, and subsequent database searching was analyzed by the Proteomics and Mass Spectrometry Services from the School of Biological Sciences in NTU, Singapore. Briefly, the excised gel band was reduced, alkylated and digested by trypsin to generate Sph1 peptides. After the peptides were extracted from the gel matrix, the peptides are enriched, and the salts and 
detergents contaminants were removed. LC-MS/MS was conducted using the Thermo QExative mass spectrometer (Thermo Fisher Scientific, USA).

\subsection{2 in-silico analysis}

To identify the putative phosphorylation sites on Sph1, as well as the cognate kinases that can act on Sph1, the full length 919 amino acid Sph1 sequence was scanned for potential phosphorylation sites and interacting kinases using two online phosphorylation prediction programmes- ScanProsite [375] and Scansite 3.0 [376]. Both prediction sites made use of the identification of short protein sequence motifs recognized by the different kinases to predict putative phosphorylation sites as well as the cognate kinases. For ScanProsite, the threshold for the scan was set to "high sensitivity" and for Scansite 3.0, the threshold for the scan was set at "high stringency".

\subsubsection{PhosTag gel}

To investigate whether Sph1 is phosphorylated by PKC, CKII and GSK3 $\beta$, we measured the mobility shift of Sph1 under kinase inhibitions using the PhosTag gel technique. This technique involves the addition of $\mathrm{M} 2+$ metal ions ( $\mathrm{M}$ can be either zinc or manganese) that can bind to the phosphate ion, followed by the addition of the dinuclear metal complex PhosTag that recognizes and binds to the M2+-phosphorylated residues. This binding interaction will significantly enhance the molecular weight of phosphorylated protein, and induces an upward electromobility shift of the protein during gel electrophoresis. The magnitude of the electromobility shift will indicate the extent of phosphorylation on the protein [377].

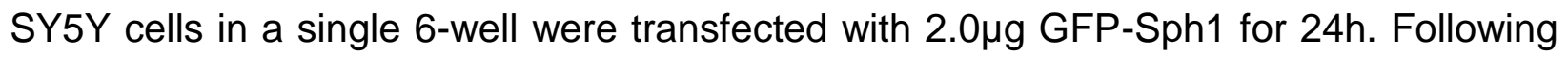
this, the transfection media was replaced with $\mathrm{S}+$ media (basal condition) or $\mathrm{S}+$ media in the presence of $2 \mu \mathrm{M}$ proteasome inhibitor MG132 for $16 \mathrm{~h}$. The extent of phosphorylation on Sph1 under basal and proteasomal stress conditions were examined by further 
addition 10nM okadaic acid (OA) to preserve all the phosphorylation sites on Sph1. By comparing the electromobility of Sph1 in absence and presence of OA under the respective basal and proteasomal stress condition, it will provide an indication on the extent of phosphorylation on Sph1 under each condition. The effects of kinase inhibitions on the Sph1 phosphorylation was examined by further treating the cells with $1.0 \mu \mathrm{M}$ PKC

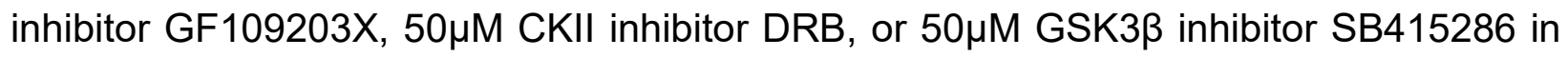
the presence of $O A$ under basal and proteasomal stress conditions. By comparing the extent of downward electromobility shift of Sph1 in the absence or presence of kinase inhibitions under OA treatment will indicate whether PKC, CKII and/or GSK3 $\beta$ phosphorylate Sph1.

Post-treatments, the cells were lysed in RIPA buffer supplemented with 6M urea to extract the total Sph1 protein pool. The proteins were separated in a $6 \%$ acrylamide gel containing 25mM PhosTag acrylamide reagent (AAL107, Wako) and $50 \mathrm{mM} \mathrm{MnCl2}$ (63535, Sigma) to amplify the extent of the electromobility shift (if present) for easier detection of the shift (as compared to higher percentage gel with lower separating resolution for GFP-Sph1). Following gel electrophoresis and electrotransfer as described in section 2.4.2, the membrane was immunoblotted with anti-GFP to investigate if kinase inhibitions affected the mobility shift of GFP-Sph1. 


\subsection{ASSAYS TO INVESTIGATE SYNPHILIN-1 AGGREPHAGY MECHANISMS}

\subsubsection{Formation and clearance of Sph1 inclusion bodies under kinase inhibitions}

The formation and autophagic susceptibility of the Agg and Agm inclusions under kinase inhibitions were with IF as previously described [18]. The influence of PKC, CKII and GSK3 $\beta$ Sph1 Agg and Agm formation was examined by transfecting SY5Y cells seeded onto coverslip in a single 24 -well and transfected with $0.5 \mu$ gFP-Sph1 for $24 \mathrm{~h}$. The cells were further treated with $2 \mu \mathrm{M}$ proteasome inhibitor MG132 for $16 \mathrm{~h}$. Following up, the

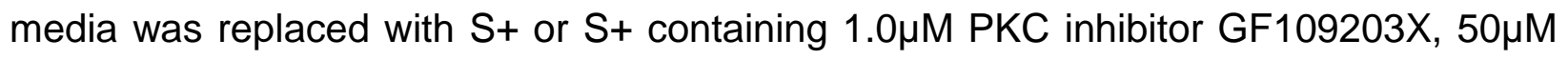
CKII inhibitor DRB, or $50 \mu \mathrm{M}$ GSK3 $\beta$ inhibitor SB415286 for $12 \mathrm{~h}$. Post-treatments, the cells were directly visualized as described in section 2.5. The percentage of transfected cells containing Agg and Agm under the different conditions were quantified.

To analyze if kinase inhibitions influence Sph1 basal aggrephagy, the cells were recovered in S+ media in the absence or presence of $10 \mathrm{mM} 3-\mathrm{MA}$, and with the addition

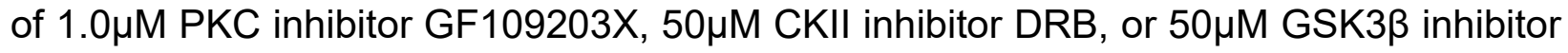
SB415286 for $12 \mathrm{~h}$. To analyze the effect on inducible autophagy, the cells were allowed to recover in either $S_{+}$or serum-free $S_{-}$- media in the absence or presence of the respective kinase inhibitor for $12 \mathrm{~h}$. Post-treatment, the percentage of cells containing Sph1 Agg and Agm were quantified under each condition. At least 100 transfected cells were analyzed.

\subsubsection{Analysis of the presence of various protein kinases in Sph1 inclusions via colocalization studies}

The association of the different protein kinases with Sph1 Agg and Agm inclusions were analyzed via IF colocalization analysis by overexpressing the differently tagged protein kinases with GFP-Sph1. SY5Y cells were co-transfected with $1.5 \mu \mathrm{g}$ GFP-Sph1 and $0.5 \mu \mathrm{g}$ 
HA-PKC $\alpha, H A-P K C y$, myc-CKII $\beta$ or HA-GSK3 $\beta$ in a single 6-well for $24 \mathrm{~h}$. Following that, the media was replaced with $S+$ containing $2 \mu \mathrm{M}$ proteasomal inhibitor MG132 $16 \mathrm{~h}$. The cells were then stained with the corresponding primary antibodies (anti-HA or anti-myc) and imaged as described in section 2.5. The Agg and Agm containing cells were identified, and the pattern as well as the frequency of colocalization between the kinase and Sph1 Agg and Agm were quantified. For the frequency, the percentage of the Agg or Agm population colocalized with the kinase was quantified.

\subsubsection{Immunoprecipitation (IP) to evaluate ubiquitination on Sph1}

To investigate whether kinase inhibitions influence the ubiquitination level of Sph1, an overexpression system was used to examine the levels of HA-tagged ubiquitination on GFP-Sph1 under kinase inhibitions during proteasomal stress. SY5Y cells in a single 6well were co-transfected with $2.0 \mu \mathrm{g}$ of GFP-Sph1 and $0.5 \mu \mathrm{g}$ of HA-WT ubiquitin or HA$\mathrm{K} 63 \mathrm{U}$ for $24 \mathrm{~h}$. Post-transfection, the transfected media was removed and replaced with $\mathrm{S}+$ media containing $2 \mu \mathrm{M}$ of proteasome inhibitor MG132 for $16 \mathrm{~h}$. After this, the media

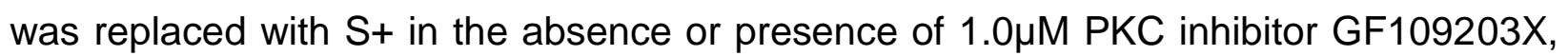

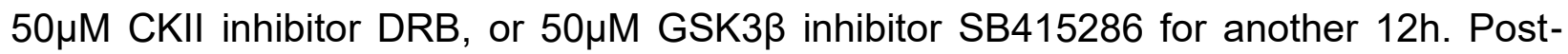
treatment, SY5Y cells were lysed in RIPA buffer supplemented with $6 \mathrm{M}$ urea. $20 \mu \mathrm{l}$ of GFP-TRAP agarose beads were added into the protein lysate followed by continuous mixing for $2 \mathrm{~h}$ at $4^{\circ} \mathrm{C}$. The unbound protein lysate was removed via centrifugation at $2500 \mathrm{~g}$ for 2 mins. The protein lysate-bound beads were then washed with the RIPA buffer three times, and the bound GFP-Sph1 proteins were released from the beads by addition of $2 x$ sample buffer followed by boiling for 10 mins. The entire eluted proteins were separated by SDS-PAGE as described in section 2.4.2 and immunoblotted for GFP and HA. The levels of ubiquitination on Sph1 under the different conditions was examined by analyzing the levels of HA-tagged ubiquitin on the immunoprecipitated GFP-Sph1. 


\subsubsection{Co-immunoprecipitation (co-IP) to investigate the interactions between Parkin and Sph1 with protein kinases}

The interactions between Sph1 and the various kinases under basal and proteasomal stress conditions were investigated in SY5Y cells using an overexpression system by coexpressing GFP-Sph1 and the differentially tagged protein kinases. SY5Y cells were cotransfected with $1.5 \mu \mathrm{g}$ GFP-Sph1 and $0.5 \mu \mathrm{g}$ of HA-PKCa, HA-PKCy, myc-CKII $\beta$ or HAGSK3 $\beta$ in a single 6-well for $24 \mathrm{~h}$. To examine whether Parkin interacts with the kinases, mCherry-Parkin stable SY5Y cells were transfected with $0.5 \mu \mathrm{g}$ of the respectively kinases in a single 6 -well for $24 \mathrm{~h}$. Subsequently, the media was removed and replaced with $\mathrm{S}_{+}$ media or S+ containing $2 \mu \mathrm{M}$ proteasome inhibitor MG132 for $16 \mathrm{~h}$. The cells were then lysed in mild detergent condition with $1 \%$ NP-40 to preserve the protein interactions.

$20 \mu \mathrm{l}$ GFP-TRAP agarose beads (to immunoprecipitate GFP-Sph1), or $1 \mu \mathrm{g}$ mCherry antibody (to immunoprecipitate mCherry-Parkin) was added to the protein lysate and allowed to mix with the lysate for $2 \mathrm{~h}$ or overnight respectively at $4^{\circ} \mathrm{C}$. For GFP-Sph1 proteins bound to the GFP-TRAP agarose beads, Sph1 was released from the beading by addition of the $2 x$ sample buffer followed by boiling for 10 mins. For proteins bound to the mCherry antibody, further $40 \mu$ protein sepharose $A$ beads were added to bind to the immunoprecipitated mCherry-Parkin in the lysate. The sepharose $A$ beads were allowed to mix with the lysate for $2 \mathrm{~h}$ at $4^{\circ} \mathrm{C}$. The mCherry-Parkin proteins bound to the antibodysepharose $A$ bead complex were then released likewise via addition of the $2 x$ sample buffer and boiling for 10 mins. beads

To examine the interactions between the kinases and Sph1, GFP-Sph1 was immunoprecipitated with anti-GFP and immunoblot for anti-HA or myc under the various kinase inhibition. To examine the interactions between the kinases and Parkin, the mCherry-Parkin was immunoprecipitated with anti-mCherry and immunoblot for anti-HA or myc. 


\subsection{OXYBLOT ASSAY TO MONITOR PROTEIN OXIDATION}

To evaluate if PE protects against protein damage under oxidative stress, the levels of oxidized proteins under $\mathrm{PE}$ and $\mathrm{H}_{2} \mathrm{O}_{2}$ treatments were determined using OxyBlot Protein Oxidation Detection Kit (S7150, Millipore) following manufacturer's protocol. SY5Y cells were treated with $4 \mathrm{mM} \mathrm{H}_{2} \mathrm{O}_{2}$ in the absence or presence of 150 and $300 \mu \mathrm{g} / \mathrm{ml} \mathrm{PE}$ for $16 \mathrm{~h}$. The cells were then lysed in RIPA buffer containing 1\% 2-mercaptoethanol, and $15 \mu \mathrm{g}$ of protein lysates were denatured with an equal volume 12\% SDS. The samples were subsequently derivatized with $1 \mathrm{X}$ DNPH solution at room temperature for $15 \mathrm{mins}$. Postderivatization, the samples were treated with neutralization solution and ready to load into a $12 \%$ SDS-PAGE gel. In the same experiment setup, the role of PE-induced autophagy under oxidative stress was also investigated by inhibiting the autophagy pathway. In the cells treated with $4 \mathrm{mM} \mathrm{H}_{2} \mathrm{O}_{2}$ and 150 or $300 \mu \mathrm{g} / \mathrm{ml} \mathrm{PE}$, another setup in the presence of lysosomal inhibitors were carried out to examine the effect of autophagy inhibition the levels of oxidized proteins. 


\subsection{ASSAYS TO MONITOR MITOCHONDRIAL HEALTH AND MITOPHAGY}

\subsubsection{Mitochondrial fractionation}

Mitochondria were isolated from cells as previously described [378, 379]. Briefly, cells were washed with cold 1x PBS and lysed in homogenization medium (prepared with $75 \mathrm{mM}$ sucrose, $30 \mathrm{mM}$ Tris $-\mathrm{HCl}, 0.1 \mathrm{mM}$ EDTA, $225 \mathrm{mM}$ mannitol, $\mathrm{pH} 7.4$ ) followed by $5 \mathrm{~min}$ centrifugation at $1000 \mathrm{~g}$. The supernatant was further centrifuged at $8000 \mathrm{~g}$ for $10 \mathrm{~min}$. Upon removal of supernatant, the resultant mitochondrial pellet was resuspended in mitochondria isolation medium (prepared with $75 \mathrm{mM}$ sucrose, $5 \mathrm{mM}$ Tris- $\mathrm{HCl}, 225 \mathrm{mM}$ mannitol, $\mathrm{pH}$ 7.4). Total lysed cells prior to separation were analyzed as total homogenate.

\subsubsection{Quantification of mitochondrial morphology}

The effects of PE on mitochondrial morphologies were analyzed by staining the mitochondria with anti-TOMM20 and visualizing the mitochondrial network as described in section 2.5. Several mitochondrial morphologies are distinguishable under inverted microscopy, including the interconnected network, tubular structures, as well as the fragmented morphology under proteasomal stress [233]. Hence, we adopted the same method for rapid identification of mitochondrial morphologies under the different treatment conditions.

SY5Y cells were treated with DMSO, $10 \mu \mathrm{M}$ CCCP, 150 or $300 \mu \mathrm{g} / \mathrm{ml} \mathrm{PE}$. The cells were stained with anti-TOMM20 to highlight the mitochondrial structure. The morphology types were characterized as "tubular/interconnected", "fragmented" or "donut" shape. The percentage of cells harboring the respectively mitochondrial morphology was tabulated. 


\subsubsection{Quantification of LC3, PINK1 and Parkin colocalization per mitochondria content}

The effect of PE on the association between the mitochondria and mitophagy machinery under basal condition and mitochondrial stress was examined by quantifying the colocalization between the organelle and LC3, PINK1 or Parkin. SY5Y cells were treated with DMSO, 150 or $300 \mu \mathrm{g} / \mathrm{ml} \mathrm{PE}$, either in the absence or presence of $10 \mu \mathrm{M} \mathrm{CCCP}$ for $16 \mathrm{~h}$ to analyze the colocalization between mitochondria and LC3 or PINK1. mCherryParkin stable cells were treated with $10 \mu \mathrm{M} \mathrm{CCCP}$ in the presence or absence of $300 \mu \mathrm{g} / \mathrm{ml}$ $\mathrm{PE}$ for $6 \mathrm{~h}$, and the colocalization between the mitochondria and mCherry-Parkin was examined. Post-treatments, the cells were co-stained with anti-TOMM20 and anti-LC3 or anti-PINK1. For the case of mCherry-Parkin cells, the cells were stained with antiTOMM20 only.

Prior to analysis of colocalization, the sharpness, brightness and contrast of the image were further enhanced with Microsoft powerpoint to $+50 \%,+40 \%$ and $+40 \%$ respectively to remove the background noise and improve the fluorescent signals to identify the colocalization. A colocalization will be considered with the appearance of yellow punctate due to overlap between the Alexa 488 green (TOMM20) and Alexa 555 red (LC3 or PINK1) signals. To confirm if the colocalization is real, each yellow punctate identified was checked against the positions of the red and green signals in their respective channels to confirm the overlapped pattern. The number of colocalization per cell was normalized against the mitochondrial content of the cell. The content was determined using Image J using the "Mito-Morphology" Macro as described [380]. Briefly, the cell of interest was highlighted and the image was split into its individual channels displayed as 8-bit images. The TOMM20 signal was threshold and the area was measured. To calculate the mitochondrial content, the TOMM20 area was divided against the cell area to indicate the percentage of cell area occupied by the mitochondria. At least 10 cells were analyzed per treatment condition. 


\subsubsection{CCCP-induced mitochondria disappearance and accumulation under autophagy inhibition (mitochondrial flux)}

The effect of PE on mitochondria clearance was examined by monitoring the TOMM20 levels in scrambled control and TFEB KD cells over a $12 \mathrm{~h}$ period under mitochondrial stress with $10 \mu \mathrm{M} \mathrm{CCCP} \mathrm{or} \mathrm{CCCP} \mathrm{in} \mathrm{the} \mathrm{presence} \mathrm{of} 300 \mu \mathrm{g} / \mathrm{ml} \mathrm{PE}$. The treatments were terminated at the $3 \mathrm{~h}, 6 \mathrm{~h}$ and $12 \mathrm{~h}$ timepoints, and the TOMM20 levels as each timepoint were examined via IB. The mitochondria turnover or mitophagy flux was examined by further treating the cells with autophagy inhibitors NL and vinblastine under $12 \mathrm{~h} \mathrm{CCCP}$ treatment or CCCP in the presence of PE. To allow monitoring of the mitophagy flux independent of mitochondrial biogenesis, protein synthesis was inhibited with $10 \mu \mathrm{g} / \mathrm{ml}$ $\mathrm{CHX}$.

\subsubsection{MitoTimer and MitoSox}

The effect of PE on mitochondrial health was examined by monitoring the response of MitoTimer, a reporter probe for studying mitochondrial content, age, damage and turnover [381-383]. MitoTimer is a duo-fluorescence protein targeted to the mitochondrial matrix where its fluorescence shifts irreversibly from green in newly synthesized healthy mitochondria to red in damaged mitochondria following oxidation events [381-383]. MitoTimer SY5Y stable cells were seeded onto coverslips in a 24-well plate format, and were treated with DMSO or $10 \mu \mathrm{M} \mathrm{CCCP}$ in the absence of presence of $300 \mu \mathrm{g} / \mathrm{ml} \mathrm{PE}$ for 16h. The mean "green" and "red" MitoTimer intensity levels in pixels within each cell were measured using the Zen software by highlighting the cell area using the "free-form drawing" tool and applying the "mean intensity" function. The average "green" and "red" MitoTimer signals per cell under the various conditions were quantified and plotted.

The protectiveness of PE and its associated polyphenol Lut-induced mitophagy against toxic reactive oxygen species generated by damaged mitochondria was examined using Mitosox (M36008, Invitrogen), a probe for mitochondrial superoxide. SY5Y cells were treated DMSO, $10 \mu \mathrm{M}$ CCCP, $10 \mu \mathrm{M}$ CCCP with the addition of $300 \mu \mathrm{g} / \mathrm{ml}$ PE or $100 \mu \mathrm{M}$ 
Lut, or the latter conditions in the presence of autophagy inhibitor NL. Following treatment, SY5Y cells were washed three times with pre-warmed 1x Hanks' Balanced Salt Solution (HBSS) (reconstituted from 10x HBSS with $1.3 \mathrm{ml}$ sodium bicarbonate in water), and stained with final concentration of $3.75 \mu \mathrm{M}$ MitoSox for $10 \mathrm{mins}$ at $37^{\circ} \mathrm{C}$. Cells were then washed three times with $1 \times$ HBSS, and fixed with $4 \%$ PFA. The cells were subsequently co-stained with anti-TOMM20 to highlight the mitochondria morphology. The mean MitoSox intensity per cell was measured using the Zen software similar to measurement of MitoTimer intensities.

\subsection{STATISTICAL ANALYSIS}

For all quantitative analysis, results were shown as mean + standard error mean (S.E.M). Student's t-test was used to determine statistical significance, defined by $p$-value $<0.05$. 


\section{CHAPTER 3}

\section{INTERPLAY BETWEEN PHOSPHORYLATION AND UBIQUITINATION REGULATES SYNPHILIN-1 MEDIATED AGGREPHAGY}




\subsection{BACKGROUND}

Synphilin-1 (Sph1) is an aggregation-prone protein found in Lewy bodies (LB) observed in a-synucleopathies [184]. Sph1 consists of 3 distinct domains, which include a Nterminal ANK1 domain, a coiled-coil domain and a C-terminal ANK2 domain [186]. While the physiological functions of Sph1 were proposed to mediate synaptic neurotransmission and general protein degradation in earlier studies [188, 384], recent studies showed that Sph1 also regulates metabolism [385-387] and mitochondrial degradation [204]. Further, previous study from our lab demonstrated that Sph1 also potentiates selective clearance of protein inclusions by autophagy (aggrephagy) independent of cargo adaptor proteins (Fig. 3.1) [18]. Sph1 was shown to promote the clearance of small cytosolic protein aggregates (Agg) basally (basal aggrephagy), and the large perinuclear aggresome (Agm) under cellular stress (inducible aggrephagy) [18]. Notably, K63-linked polyubiquitination (K63U) on the ANK1 domain reduces protein mobility on the surface of the Agm, and stabilizes the structure to facilitate direct assembly of the class III PI3-kinase complex on the Agm surface [18]. The class III PI3-kinase complex subsequently initiates biogenesis of autophagosomal membrane around the Agm to mediate autophagic clearance [18]. Hence, the study uncovered a novel role of Sph1 in facilitating noncanonical aggrephagy that is independent of cargo adaptor proteins. This finding has direct implication in neurodegenerative and aging conditions, where mutations and/or reduced expressions of cargo adaptor proteins were observed [12]. Sph1 expression was reported to be upregulated in the aged brain [189], thus suggesting the intriguing possibility that Sph1-mediated aggrephagy may act as an alternative mechanism to compensate for the perturbed aggrephagy observed under clinical conditions to promote aggrephagy.

Selective autophagy has been well-documented to be modulated by phosphorylation and ubiquitination [371]. Indeed, many proteins are multiply modified, and the crosstalk between post-translational modifications (PTM) regulates the behavior of the protein [388]. The activity of Sph1 is also modulated by phosphorylation and ubiquitination. CKII [201] and GSK3 $\beta$ [202] phosphorylate and enhance Sph1 aggregation propensity, 
whereas ubiquitination modifies the aggregation [202, 389] and aggrephagy [18] propensities of Sph1. Interestingly, while K63U on Sph1 is important for inducible aggrephagy, this modification is dispensable for basal clearance of Agg [18] and the role of PTM in influencing basal Agg removal remains unknown.

Hence, this section aims to investigate the molecular mechanism underlying Sph1mediated basal aggrephagy. Since phosphorylation also influences Sph1 activity [201, 202], we were keen to examine the involvement of phosphorylation in regulating the autophagic susceptibility of Agg. Additionally, the role of phosphorylation in Sph1mediated inducible aggrephagy was also examined, as crosstalk between phosphorylation and ubiquitination has been shown to influence Sph1 aggregation behavior [202]. 


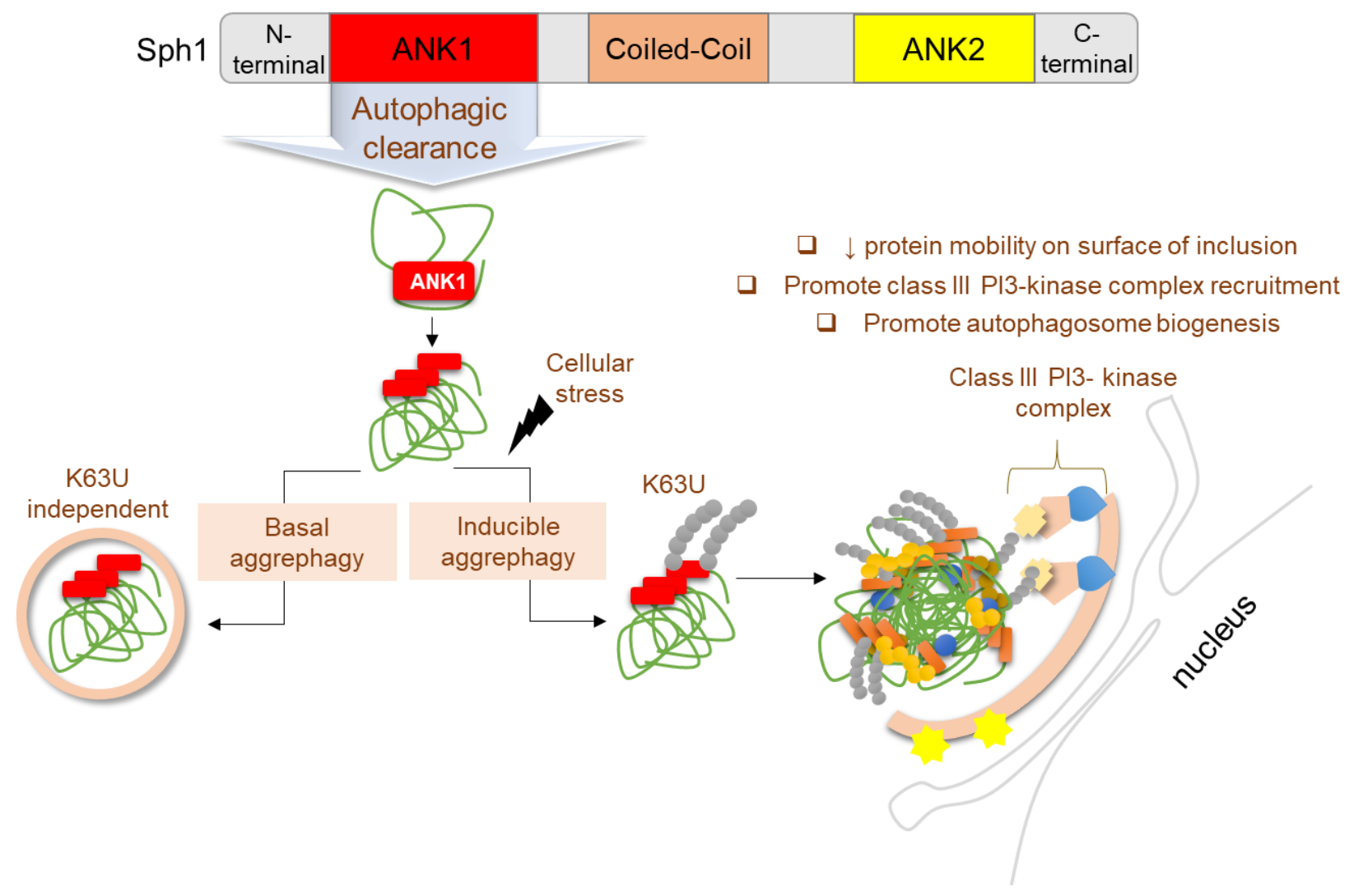

Figure 3.1 Schematic diagram depicting K63U modulation of Sph1 aggrephagy behavior. ANK1 domain of Sph1 mediates targeting of the small cytosolic protein aggregates (Agg) for constitutive turnover by basal aggrephagy, and the large perinuclear aggresome (Agm) for removal by inducible aggrephagy. K63U on the ANK1 is important for modulating the biophysical properties of Agm. K63U reduces the protein mobility on the surface of the Agm, and stabilizes the inclusion to allow direct assembly of the class III PI3-kinase on the Agm surface to initiate autophagosome biogenesis. However, K63U is dispensable for the removal of the Agg by basal aggrephagy. 


\subsection{IDENTIFICATION OF PHOSPHORYLATED SITES ON SYNPHILIN-1 BY IN-SILICO ANALYSIS AND MASS SPECTROMETRY}

To investigate whether phosphorylation regulates Sph1 aggrephagy behavior, we first examined the phosphorylation patterns of Sph1. Sph1 is phosphorylated by CKII [201] and GSK3 $\beta$ [202] that regulate its aggregation behavior. While CKII phosphorylation site on Sph1 remains unidentified, GSK3 $\beta$ phosphorylates Sph1 at S556 [202]. Additional phosphorylation sites on Sph1 at S211 and S215 have also been detected via phosphorylation motif mapping [390]. Besides these known phosphorylation sites and interacting kinases, we further queried whether there are novel phosphorylation sites on Sph1. To address this, in-silico analysis and mass spectrometry of Sph1 were performed to identify any new putative phosphorylation sites and the cognate kinases for Sph1.

\subsection{1 in-silico analysis}

The ScanProsite [375] and Scansite 3.0 [376] prediction tools were used to scan for putative phosphorylation sites on Sph1 along with the cognate kinases. Both prediction methods involve the mapping of consensus protein sequence motifs recognized by the different kinases, to predict putative phosphorylation sites and the cognate kinases on the protein of interest. For ScanProsite, the threshold for the scan was set to "high sensitivity" and for Scansite 3.0, the threshold for the scan was set to "high stringency". The comprehensive data gathered from both prediction tools is found in appendix 1 .

ScanProsite identified several putative modifications on Sph1, including phosphorylation, $\mathrm{N}$-glycosylation, N-myristoylation and amidation. For phosphorylation, 32 putative phosphorylation sites were detected, with PKC and CKII predicted as the cognate kinases (Fig. 3.2). Scansite 3.0 identified 8 putative phosphorylation sites on Sph1, along with binding motifs for GSK3, GSK3 $\beta$, polo-like kinase 1 (PLK1), AMPK, cyclin-dependent kinase 1 (CDK1) and cell division cycle protein 2 (Cdc 2) (Fig. 3.2). Interestingly, the 
putative phosphorylation sites identified by both prediction methods represent novel sites on Sph1.

Thus, the in-silico analysis suggests that Sph1 harbors additional putative phosphorylation sites and may be regulated by other kinases besides those reported. To validate the relevance of these putative sites on Sph1, mass spectrometry analysis of Sph1 was carried out. 
Putative phosphorylation sites and the corresponding cognate kinases identified by ScanProsite and Scansite prediction tools

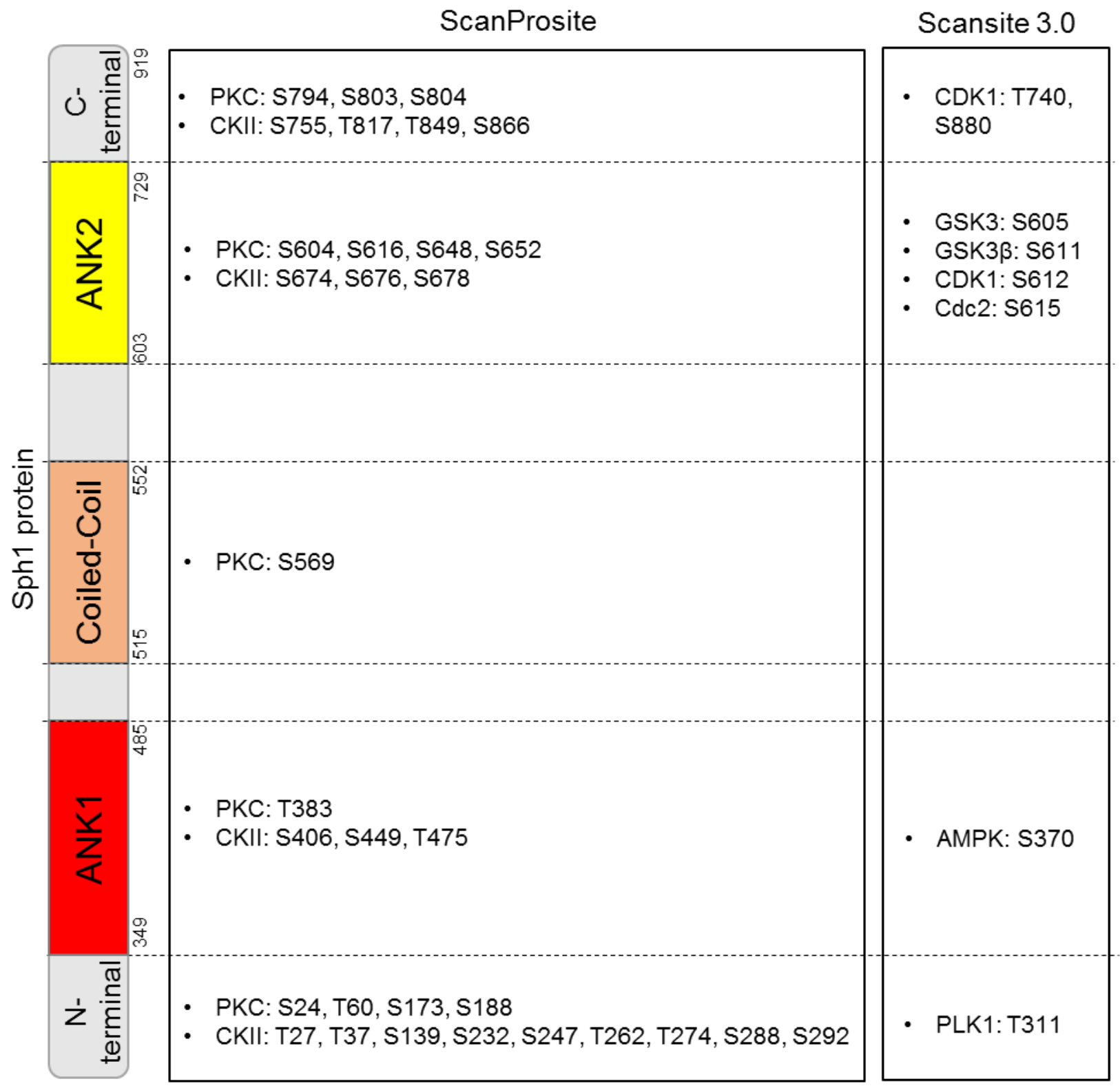

Figure 3.2 Phosphorylation sites and kinases that modify Sph1 predicted by ScanProsite and Scansite 3.0 programmes. Schematic diagram highlighting the putative phosphorylation sites and the cognate kinases predicted to phosphorylate Sph1 across the different domains. 


\subsubsection{Mass spectrometry analysis of Sph1 phosphorylation}

Mass spectrometry is a commonly used technique to identify phosphorylation sites in-vivo due to its speed, sensitivity, selectivity and throughput. Here, we analyzed the in-vivo phosphorylation patterns of Sph1 using mass spectrometry analysis. To prepare the Sph1 protein sample for mass spectrometry, SY5Y cells were transiently transfected with GFPSph1 for $48 \mathrm{~h}$. The cells were lysed in denaturing RIPA buffer containing $6 \mathrm{M}$ urea to solubilize both the soluble (cytosolic) and insoluble (aggregated) forms of Sph1. GFPSph1 were immunoprecipitated from the lysate with GFP-TRAP agarose beads, and the purified protein sample was sent for mass spectrometry analysis.

The efficiency of the GFP-TRAP agarose beads in immunoprecipitating GFP-Sph1 proteins was examined prior to large scale purification of Sph1 for mass spectrometry. Optimization experiment showed that $20 \mu$ of GFP-TRAP agarose beads successfully purified total GFP-Sph1 proteins expressed in SY5Y transfected with 2ug GFP-Sph1 in a single 6-well (Fig. 3.3a). Based on these optimized conditions, the expression and immunoprecipitation of Sph1 were scaled up accordingly to obtain $\sim 2 \mathrm{mg}$ purified GFPSph1 proteins for liquid chromatography followed by tandem mass spectrometry (LCMS/MS).

Based on the mass spectrometry results, a total of 79 unique peptides were generated after proteolytic digestion by trypsin. Among these, 13 unique peptides were shown to harbor 22 serine and threonine phosphorylation sites (Fig. 3.3b and c). The mass spectrometry results showed that the majority of the phosphorylated sites on Sph1 were found in the N-terminal and the ANK2-C-terminal regions (Fig. 3.3b). Interestingly, no phosphorylation sites were detected in ANK1, the domain shown to be important for mediating basal and inducible aggrephagy (Fig. 3.3b).

The three reported phosphorylation sites on Sph1, S211, S215 [390] and S556 [202], were also identified in our mass spectrometry analysis, validating the sensitivity and reliability of our mass spectrometry results. The remaining sites identified in this study 
thus represent novel phosphorylation sites on Sph1 in-vivo. Several of the phosphorylation sites identified by mass spectrometry were also picked up by the in-silico analysis. These include S674 (CKII), S676 (CKII) and S804 (PKC). Therefore, these novel sites and their corresponding cognate kinases CKII and PKC may play a role in modulating Sph1 functions.

Together, the in-silico and mass spectrometry analysis elucidated multiple novel phosphorylation sites on Sph1. For the subsequent studies, we focused our attention on PKC, CKII and GSK3 $\beta$ in regulating Sph1 aggrephagy behavior. Based on our analysis (Fig. 3.2), PKC may be a newly identified kinase that phosphorylates Sph1. While CKII and GSK3 $\beta$ have been shown to phosphorylate Sph1 to regulate its inclusion formation [201, 202], not much is known regarding their effects on Sph1 aggrephagy behavior. In the next part of the study, we investigated the effects of PKC, CKII and GSK3 $\beta$ on regulating Sph1 Agg and Agm autophagic susceptibility. For PKC, we analyzed the PKC $\alpha$ and $\mathrm{PKCY}$ isoforms out of the 16 known isoforms based on the following reasons: (1) PKCa is the most abundant isoform intracellularly, and (2) PKCY is a neuron-specific isoform and hence is highly relevant in our neuronal SY5Y cell model [391]. For CKII, we focused on the $\beta$ regulatory isoform as this isoform has been shown to interact with Sph1 [201]. 
a

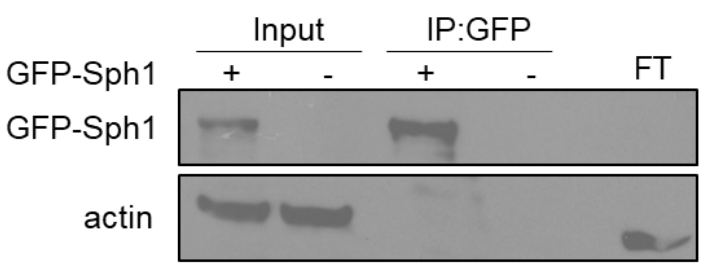

b

$\mathrm{N}-$

C-

\begin{tabular}{|c|c|c|c|c|c|c|}
\hline \multirow{2}{*}{$\begin{array}{c}\text { Number of } \\
\text { phosphorylated } \\
\text { sites }\end{array}$} & termina & & \multicolumn{3}{|r|}{ ANK2 } & terminal \\
\hline & 9 & ANK1 & $\begin{array}{l}\text { Coiled- } \\
\text { Coil }\end{array}$ & 7 & 6 & 2 \\
\hline $\begin{array}{c}\text { Phosphorylation } \\
\text { sites }\end{array}$ & $\begin{array}{c}\text { S48 } \\
\text { S80 } \\
\text { T138 } \\
\text { S139 } \\
\text { S211 } \\
\text { S215 } \\
\text { S222 } \\
\text { T233 } \\
\text { S239 }\end{array}$ & & & $\begin{array}{l}\text { S556 } \\
\text { S559 } \\
\text { S560 } \\
\text { S562 } \\
\text { S563 } \\
\text { S576 }\end{array}$ & $\begin{array}{l}\text { S674 } \\
\text { S676 } \\
\text { S682 } \\
\text { T690 } \\
\text { T691 }\end{array}$ & $\begin{array}{l}\text { S801 } \\
\text { S804 }\end{array}$ \\
\hline
\end{tabular}

C

\begin{tabular}{|c|c|c|c|}
\hline $\begin{array}{l}\text { Phospho- } \\
\text { site }\end{array}$ & Peptide & Hits & $\begin{array}{l}\text { Pep } \\
\text { Score }\end{array}$ \\
\hline S48 & 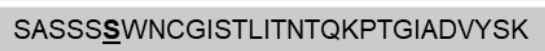 & 1 & 36.85 \\
\hline S80 & RV $\underline{S} P L K H Q P E T L E N N E S D D Q K$ & 4 & 33.37 \\
\hline T138 & SSEPSISLGELEHYDLDM\#DEILDVPYIK & 1 & 36.43 \\
\hline S139 & SSEPST́LGELEHYDLDMDEILDVPYIK & 1 & 39.16 \\
\hline S211 & ACSTGSSESSSSNMAPFCVL $\underline{S} P V K$ & 5 & 53.05 \\
\hline S215 & ACSTGSSESSSSNMAPFCVLSPVK $\underline{S} P H L R$ & 4 & 59.51 \\
\hline S222 & A $\underline{S} A V I H D Q H K L S T E E T E I S P P L V K$ & 2 & 25.84 \\
\hline T233 & ASAVIHDQHKLSIEETEISPPLVK & 1 & 60.54 \\
\hline S239 & LSTEETEISPPPLVK & 4 & 59.94 \\
\hline S556 & SEGKSLLPSSPSSPSSPASR & 2 & 46.92 \\
\hline S559 & SEGKSLPSSSPSSPSSPASR & 5 & 46.92 \\
\hline S560 & 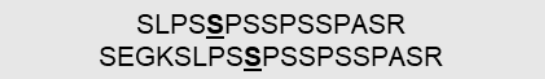 & $\begin{array}{l}3 \\
4\end{array}$ & $\begin{array}{l}79.14 \\
31.04\end{array}$ \\
\hline S562 & 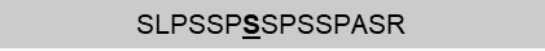 & 8 & 54.9 \\
\hline S563 & SLPSSPSㅁPSSPASR & 10 & 79.14 \\
\hline S576 & SQWKSPPDADDDSVAK & 6 & 44.55 \\
\hline S674 & sLSESDTDSNNSEDPKTTPVRK & 1 & 69.68 \\
\hline S676 & SLISESDTDSNNSEDPK & 6 & 69.68 \\
\hline S682 & SLSESDTDS్NNSEDPK & 5 & 60.78 \\
\hline T690 & SLSESDTDSNNSEDPKITPVR & 4 & 62.85 \\
\hline T691 & SLSESDTDSNNSEDPKTIPVR & 5 & 46.51 \\
\hline S801 & SALKSPSSK & 18 & 46.95 \\
\hline S804 & SALKSPSㅗK & 3 & 21.48 \\
\hline
\end{tabular}


Figure 3.3 Mass spectrometry analysis detected multiple phosphorylation sites on Sph1. SY5Y cells were transiently transfected with GFP-Sph1 for $48 \mathrm{~h}$. The cells were lysed in RIPA buffer with $6 \mathrm{M}$ urea, and the lysate was subjected to immunoprecipitation (IP) with GFP-TRAP agarose beads. (a) Immunoblot for the total expression and immunoprecipitated levels of GFP-Sph1. (b) Diagram showing the phosphorylated sites on Sph1 detected by mass spectrometry. (c) Table shows the peptides that harbor the phosphorylation sites generated from the trypsin proteolytic cleavage in mass spectrometry analysis. The number of times (hit) these phosphorylated sites were identified in the peptides and the confidence value (pep score) for each site were indicated. 


\subsection{SYNPHILIN-1 ASSOCIATES WITH PKC AND GSK3 $\beta$ ISOFORMS}

To investigate whether PKCa, PKCy, CKII $\beta$ and GSK3 $\beta$ interact and phosphorylate Sph1 in our study, co-expression of HA-PKC $\alpha$, HA-PKCy, myc-CKII $\beta$ and HA-GSK3 $\beta$ individually with GFP-Sph1 were carried out to assess their respective interactions with Sph1 under basal and proteasomal stress conditions via co-immunoprecipitation (co-IP) and colocalization analysis.

\subsubsection{Sph1 interacts with PKC $\alpha$, PKC $y$ and GSK3 $\beta$}

First, the potential interactions between Sph1 and the kinases were evaluated by co-IP. Briefly, SY5Y cells co-transfected with GFP-Sph1 and the individual kinase isoform were left either untreated or treated with MG132 proteasomal inhibitor under basal serum containing $\left(\mathrm{S}_{+}\right)$condition. GFP-Sph1 was then immunoprecipitated with GFP-TRAP agarose beads and immunoblotted with either anti-HA or anti-myc antibody to probe for signs of co-immunoprecipitating kinases. The co-IP experiments were performed using $1 \%$ NP-40 mild detergent lysis buffer to preserve molecular interactions. This lysis condition mainly extracts the soluble, cytosolic pool of Sph1. Hence, the co-IP analyzed interactions between the soluble forms of Sph1 and the kinases. Although assessing the interactions in the insoluble fraction of Sph1 is more relevant for aggrephagy study, it is technically challenging to analyze protein interactions under harsh lysis condition used to extract insoluble proteins. Further, examining the interactions of soluble Sph1 with the kinases may provide insights on whether Sph1 interacts with the kinases at an early stage prior to inclusion formation.

PKC $\alpha$ and PKCy. PKCa co-immunoprecipitated with Sph1 under proteasomal inhibition with MG132, while negligible interaction was observed under basal S+ condition (Fig. 3.4a). On the other hand, PKCy co-immunoprecipitated with Sph1 under basal S+ condition, and the interaction was also preserved under proteasomal stress (Fig. 3.4b). 
These results show that Sph1 interacts with PKCa mainly in response to proteasomal stress, while it interacts with PKCY both basally and under stress condition.

CKII $\beta$. CKII has been reported to phosphorylate Sph1, and the $\beta$ subunit is responsible for the interaction of the CKII complex with Sph1 [201]. In our study, we encountered a consistent problem of low myc-CKII $\beta$ expression under basal S+ condition (Fig. 3.4c, input). Proteasomal inhibition with MG132 on the other hand, elevated the expression of myc-CKII $\beta$, possibly by stabilizing the protein against degradation. Hence, the interaction between Sph1 and CKII $\beta$ could only be examined under proteasomal stress condition in our study. However, contradictory to reported finding [201], CKII $\beta$ did not coimmunoprecipitate with Sph1 upon MG132 treatment (Fig. 3.4c). This highlights that CKII $\beta$ does not interact with Sph1 under proteasomal stress in our study.

GSK3 $\beta$. Similar to CKII $\beta$, the expression of HA-GSK3 $\beta$ was lower under basal $\mathrm{S}^{+}$ condition as compared to proteasomal inhibition condition (Fig. 3.4d, input). However, unlike CKII $\beta$, GSK3 $\beta$ co-immunoprecipitated with Sph1 upon MG132 treatment (Fig. $3.4 d)$.

Collectively, our co-IP results demonstrate that Sph1 interacts with $\mathrm{PKC}$ y under basal and proteasomal stress conditions, and with PKCY and GSK3 $\beta$ only in response to proteasomal stress. These interaction patterns suggest that $\mathrm{PKCY}$ may constitutively phosphorylate Sph1, but PKCa and GSK3 $\beta$ only phosphorylate Sph1 upon onset of proteasomal stress. Thus, PKCY and GSK3 $\beta$ may play an instrumental role in modulating Sph1 behavior under proteasomal stress. 
a
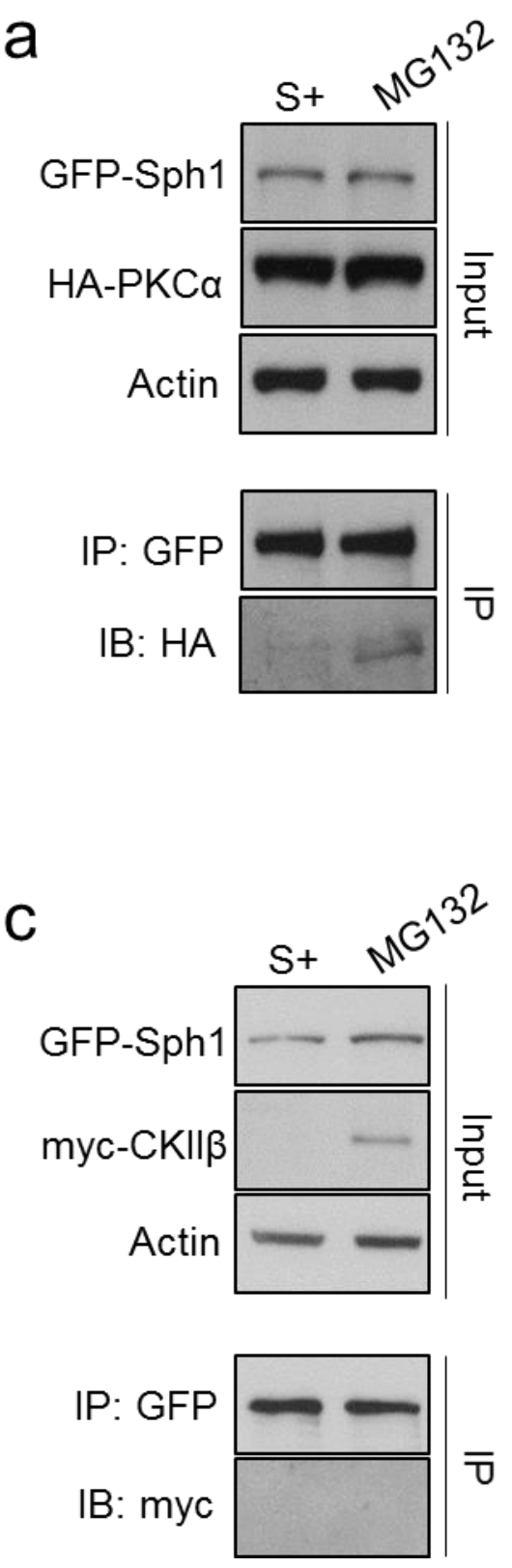

b

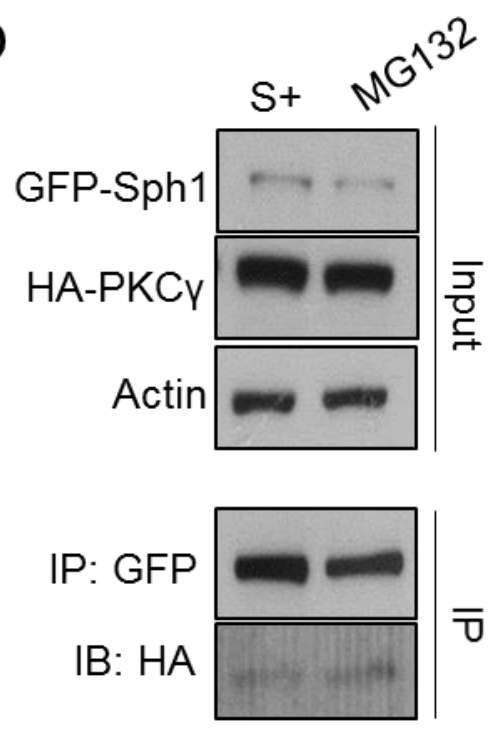

d

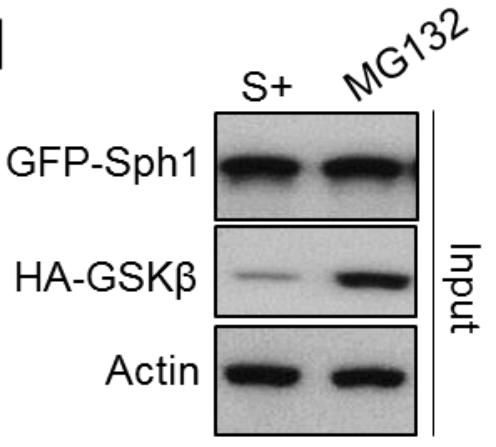

IP: GFP

IB: HA

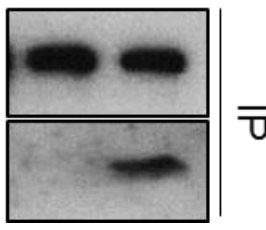


Figure 3.4 PKCY interacts with Sph1 basally and under proteasomal stress, but PKC $\alpha$ and GSK3 $\beta$ interact with Sph1 in response to proteasomal stress. SY5Y cells were transiently co-transfected with GFP-Sph1 and the individual kinase isoform represented in the figure by (a) HA-PKCa, (b) HA-PKCy, (c) myc-CKII $\beta$ and (d) HAGSK3 $\beta$ for $24 \mathrm{~h}$. The media was then refreshed with serum-rich $\mathrm{S}+$ media alone or supplemented with $2 \mu \mathrm{M}$ MG132 proteasomal inhibitor for $16 \mathrm{~h}$. Cells were lysed with $1 \%$ NP-40 lysis buffer, and the lysates were subjected to immunoprecipitation (IP) with GFPTRAP agarose beads, followed by immunoblotting (IB) with either anti-HA or anti-myc antibody. The upper panel (input) shows the total expression of GFP-Sph1 and the respective kinase isoform in the lysate. The lower panel (IP) shows the levels of GFPSph1 immunoprecipitated, and the levels of the individual kinase isoform that coimmunoprecipitated with GFP-Sph1. The co-IP experiments were repeated 3 times $(n=3)$. 


\subsubsection{PKC $\alpha$, PKC $y$, CKII $\beta$ and GSK3 $\beta$ localized to Agg and Agm formed by Sph1}

To analyze if the interactions between soluble Sph1 and the kinases in the cytosol (Fig. 3.4) are preserved in the inclusions formed by Sph1, colocalization of various kinases with Sph1 Agg and Agm were examined. Based on the reported studies [18, 205], Sph1 forms multiple, small cytosolic Agg of approximately $0.5-2.5 \mu \mathrm{m}$ in diameter, while the Agm appears as a large perinuclear inclusion approximately 5-8 $\mu \mathrm{m}$ in diameter (Fig. 3.5a). In our study, we observed that GFP-Sph1 formed small cytosolic Agg inclusions as well as large perinuclear Agm inclusions when overexpressed in SY5Y cells (Fig. 3.5a) as previously reported (Fig. 3.5a) [18].

When overexpressed basally, Sph1 predominantly formed more Agg ( 20\%) than Agm ( 10\%) (Fig. 3.5b). To increase the Agm pool for colocalization analysis with the kinases, the cells were treated with proteasomal inhibitor MG132 [27, 28]. Indeed, we observed a significant increase in the percentage of cells containing Agm from approximately $10 \%$ to 20\% upon MG132 treatment (Fig. 3.5b). GFP-Sph1 colocalization studies with overexpressed $\mathrm{HA}-\mathrm{PKC} \alpha$ and $\mathrm{HA}-\mathrm{PKC} \gamma$ showed that both PKC kinase isoforms localized to Sph1 Agm in two distinct patterns. In $~ 65 \%$ co-stained Agm, both PKC isoforms colocalized with Sph1 Agm as a ring-like PKC structure (Ring), while 20\% co-stained Agm showed complete colocalization (Full) (Fig. 3.5c). For overexpressed myc-CKIIß, only 45\% GFP-Sph1 Agm showed colocalization with the kinase and mainly as a ringlike structure around the inclusion (Fig. 3.5c). Indeed, CKII $\beta$ has been reported to form a "halo-ring" around the Agm-like Lewy bodies in the dopaminergic neurons of PD patients [201]. Colocalization analysis of overexpressed HA-GSK3 $\beta$ with GFP-Sph1 showed GSK3 $\beta$ localized to Agm in the same pattern as the PKC isoforms (Fig. 3.5c). Approximately $70 \%$ co-stained Agm were surrounded by a ring-like structure formed by GSK3 $\beta$, while 25\% Agm colocalized completely with GSK3 $\beta$ (Fig. 3.5c).

Next, the association between the Agg formed by Sph1 and the kinases was examined. We observed that HA-PKC $\alpha, \mathrm{HA}-\mathrm{PKC} \gamma$, myc-CKII $\beta$ and HA-GSK3 $\beta$ localized to the Agg, albeit in different proportions (Fig. 3.5d). Approximately 30\% and 37\% Sph1 Agg 
colocalized with PKC $\alpha$ and PKC $\gamma$ respectively, while only $5 \%$ and $20 \%$ colocalization was detected for CKII $\beta$ and GSK3 $\beta$ respectively. Notably, CKII $\beta$ similarly localized to the Agg as a ring-like structure surrounding the Agg inclusion (Fig. 3.5d).

Collectively, the localization of PKC $\alpha, P K C \gamma, C K I I \beta$ and GSK3 $\beta$ to the Agg and Agm formed by Sph1 provided another supporting evidence that PKC, CKII and GSK3 $\beta$ may potentially influence Sph1 phosphorylation and aggrephagy behavior. Interestingly, the presence of myc-CKII $\beta$ in Agg and Agm does not seem to agree with earlier observation that CKII $\beta$ does not interact with Sph1 (Fig. 3.4C). A plausible explanation is that CKIIß may associate with the various inclusions after the formation, hence the interaction between Sph1 and CKII $\beta$ could not be detected in their soluble states with the co-IP assay. 
a
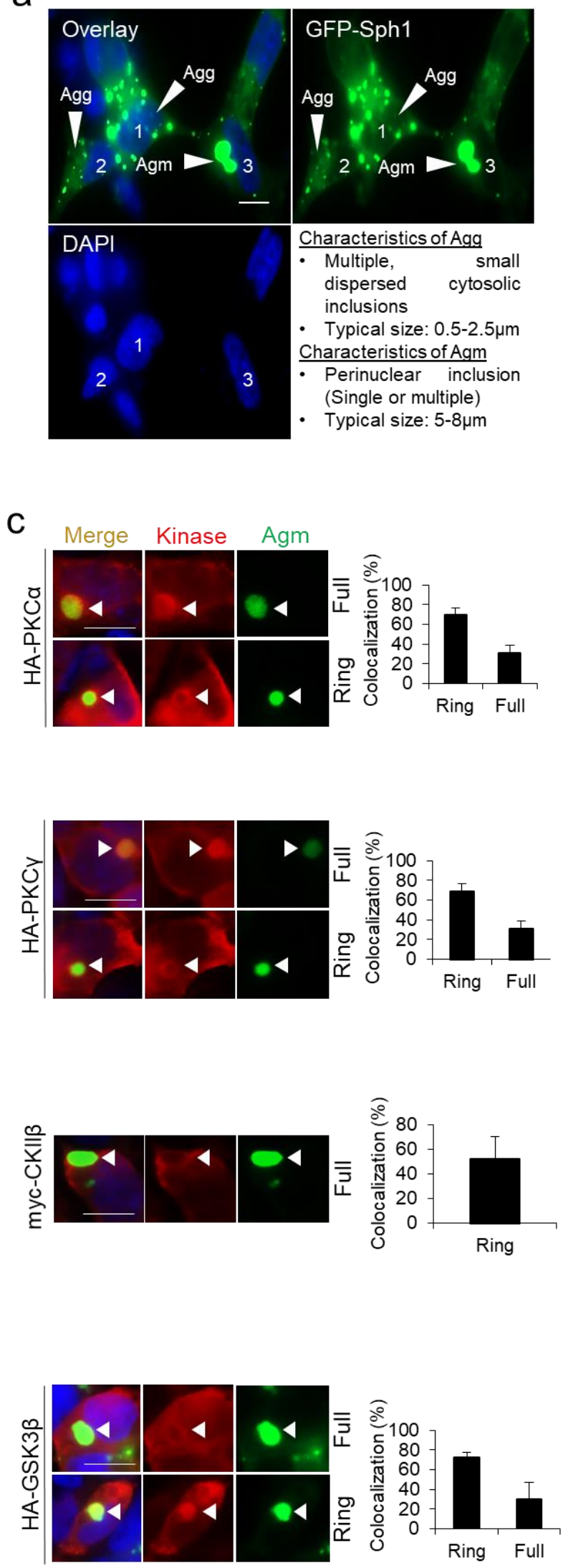

b
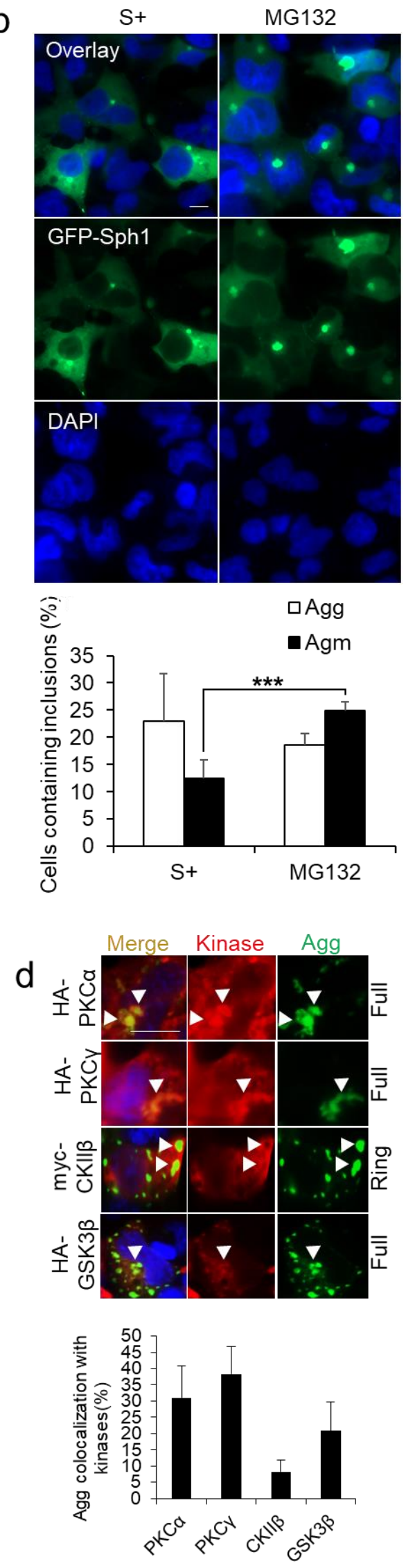
Figure 3.5 PKC $\alpha$, PKCY, CKII $\beta$ and GSK3 $\beta$ localized to the Agg and Agm formed by Sph1. (a) SY5Y cells were transiently transfected with GFP-Sph1 under basal S+ condition for 24h. The white arrows highlight the Agg (arrows 1 and 2) and Agm (arrow 3) formed by GFP-Sph1. The features used to identify the Agg and Agm were described. (b) Levels of Agg and Agm formed by GFP-Sph1 basally and under proteasomal stress. SY5Y cells were transiently transfected with GFP-Sph1 for 24h, and the media was refreshed with serum-rich $S_{+}$media, or with the addition of $2 \mu \mathrm{M}$ proteasomal inhibitor MG132 for 16h. (Top) Immunofluorescence images of the Agg and Agm formed under the different conditions. (Bottom) Quantification of the percentage of Agg and Agm containing cells under the different conditions. (c-d) Localization of HA-PKC $\alpha, \mathrm{HA}-\mathrm{PKC} \gamma$, myc-CKII $\beta$ and HA-GSK3 $\beta$ to the (c) Agm and (d) Agg formed by GFP-Sph1. SY5Y cells were transiently co-transfected with GFP-Sph1 and the individual kinase isoform for $24 \mathrm{~h}$, followed by treatment with $2 \mu \mathrm{M}$ proteasomal inhibitor MG132 for 16h. (Left) Immunofluorescence images for the localization pattern of the individual kinase to the Agm and Agg formed by Sph1. The cells were stained with anti-HA or anti-myc to identify the kinase localization with GFP-Sph1. (Right) Quantification of the different types of kinase localization pattern with the Agm and Agg. At least 50 cells from random fields were analyzed for each condition. Nuclei were stained with DAPI. All values are mean + S.E.M ( $n=3)$. Differences against $S+$ control are significant for ${ }^{* * *} p<0.005$. Scale bars, $10 \mu \mathrm{m}$. 


\subsection{SYNPHILIN-1 IS HIGHLY PHOSPHORYLATED UNDER PROTEASOMAL STRESS}

With the observations that PKC, CKII and GSK3 $\beta$ isoforms associate with Sph1 (Fig. 3.4 and 3.5), we next examined if these kinases phosphorylate Sph1 under basal and proteasomal stress condition using the PhosTag SDS-PAGE gel analysis technique.

PhosTag gel analysis involves the addition of $\mathrm{M} 2+$ metal ions ( $\mathrm{M}$ can be either zinc or manganese) that can bind to the phosphate ion, followed by the addition of the dinuclear metal complex PhosTag that recognizes and binds to the M2+-phosphorylated residues. This binding interaction will significantly enhance the molecular weight of phosphorylated protein, and induces an upward electromobility shift of the protein during gel electrophoresis. The magnitude of the electromobility shift will indicate the extent of phosphorylation on the protein [377].

Briefly, SY5Y cells were transiently transfected with GFP-Sph1 under basal S+ condition in the absence or presence of proteasomal inhibitor MG132. Next the transfected cells were treated with chemical inhibitors of PKC, CKII and GSK3 $\beta$ respectively to determine the effects of inhibiting these kinases on Sph1 phosphorylation. The inhibitors include

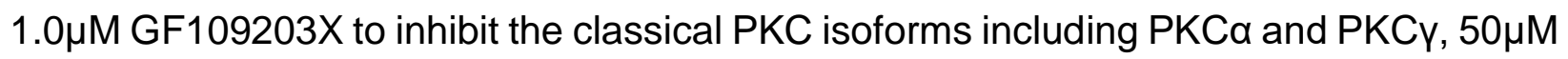
5,6-dichloro-1-beta-D-ribofuranosylbenzimidazole (DRB) to perturb the interaction between CKIl $\alpha$ and $\beta$ isoforms and the formation of functional CKII complex, and $50 \mu \mathrm{M}$ SB415286 to compete with ATP binding on GSK3 $\beta$ and disrupt the catalytic activity of GSK3 $\beta$. The effects of kinase inhibitions on Sph1 electromobility were analyzed via PhosTag gel analysis. Phosphatase inhibitor okadaic acid $(\mathrm{OA})$ were added to preserve the highly labile phosphorylation modifications on Sph1.

Under basal S+ condition, addition of OA did not alter the electromobility of GFP-Sph1 protein as compared to the untreated control (Fig. 3.6a). Further addition of the kinase inhibitors GF109203X, DRB and SB415286 under OA treatment also did not significantly 
alter the electromobility of Sph1 (Fig. 3.6a). Previous studies have demonstrated that CKII and GSK3 $\beta$ phosphorylate Sph1 [201, 202]. On the contrary, no Sph1 electromobility changes were observed upon CKII and GSK3 $\beta$ inhibitions with DRB and SB415286 respectively in our study. A plausible explanation is that Sph1 may be weakly phosphorylated under basal $\mathrm{S}+$ condition. In this case, the PhosTag gel technique may not be sensitive enough to detect small changes in the phosphorylation signals as compared to the phospho-autoradiography method used in the published studies [377].

In contrast, under proteasomal inhibition with MG132, an obvious upward shift of the GFP-Sph1 band was observed in the presence of phosphatase inhibitor OA as compared to the untreated control (Fig. 3.6b). This result shows that Sph1 is more prominently phosphorylated under proteasomal stress than basally. Notably, further addition of all three kinase inhibitors individually under $O A$ treatment reduced the electromobility of Sph1, causing a downward shift in the Sph1 band as compared to the OA treated condition without kinase inhibition (Fig. 3.6b). Among the three kinases, CKII and GSK3 $\beta$ inhibitions led to a much greater downward shift of the GFP-Sph1 band than PKC inhibition (Fig. 3.6b). This result shows that CKII and GSK3 $\beta$ phosphorylate Sph1 to a greater extent than PKC.

Taken together, the PhosTag gel analysis revealed that PKC, CKII and GSK3 $\beta$ phosphorylate Sph1 mainly in response to proteasomal stress. The varying extent of phosphorylation by the kinases suggests that they may contribute differently to regulating Sph1 function during proteasomal stress. 


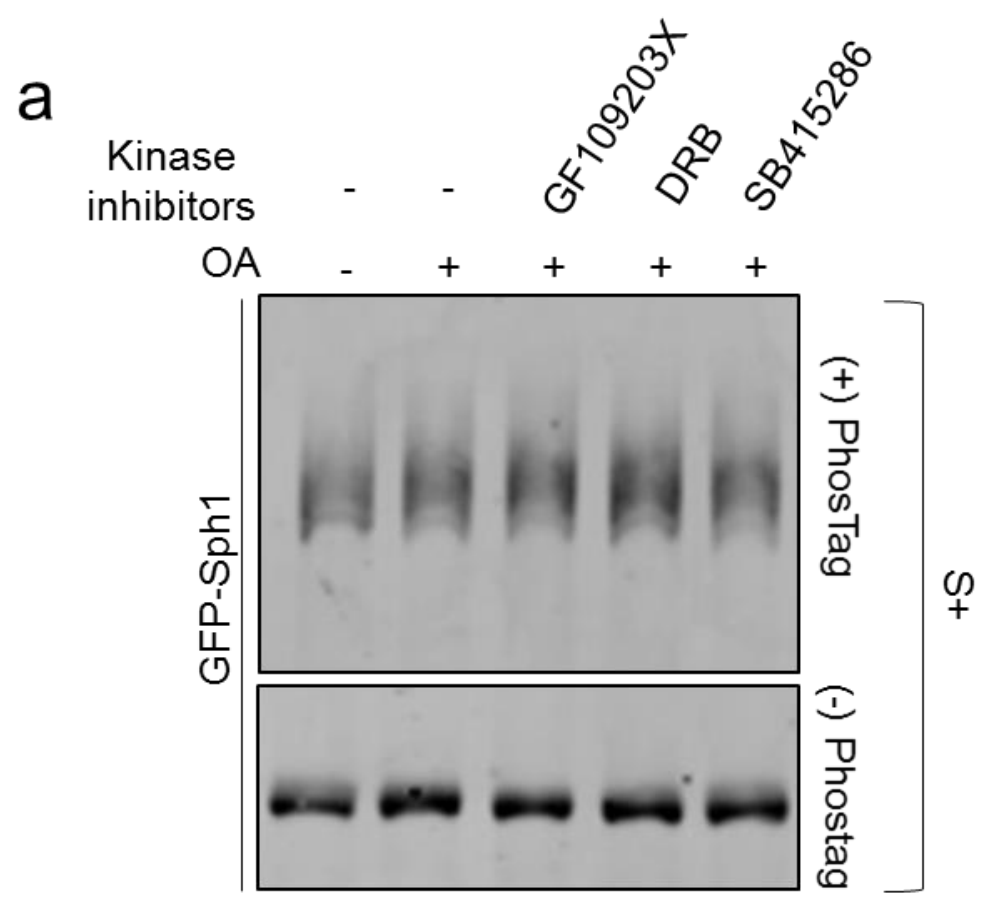

b

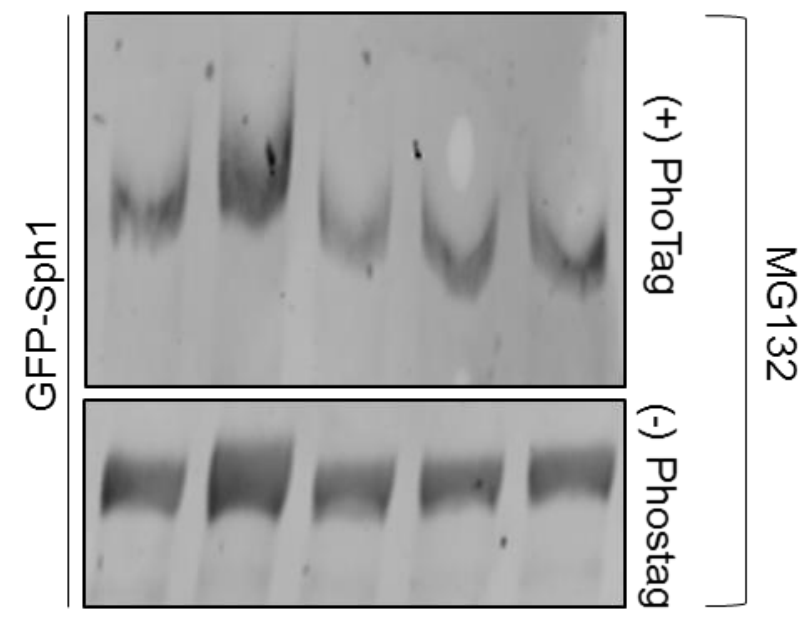


Figure 3.6 Inhibitions of PKC, CKII and GSK3 $\beta$ differentially modulate phosphorylation of Sph1 under proteasomal stress. Immunoblots of GFP-Sph1 in a $6 \%$ 25mM PhosTag gel ((+) phostag) and normal 6\% SDS-PAGE gel ((-) phostag). Phosphorylation profiles of GFP-Sph1 transiently transfected in SY5Y cells under (a) basal serum-rich $S_{+}$condition or (b) proteasomal inhibition with $2 \mu \mathrm{M}$ MG132 were analyzed. GFP-Sph1 transfected cells were further left untreated or treated with 10nM

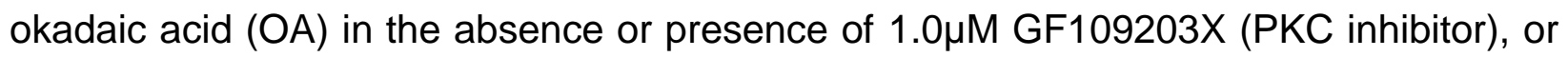

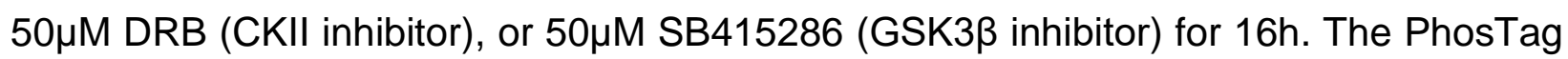
assay was repeated 3 times $(n=3)$. 


\subsection{PHARMACOLOGICAL AND GENETIC INHIBITION OF PKC, CKII AND GSK3 $\beta$ ON SYNPHILIN-1 PROTEIN EXPRESSION, AGGREGATION AND AGGREPHAGY PROPENSITIES}

Following the observation that Sph1 is phosphorylated by PKC, CKII and GSK3 $\beta$, the influence of these kinases on Sph1 aggrephagy behavior was examined. Specifically, the effects of inhibiting the various kinases on basal and inducible clearance of Sph1 Agg and Agm were assessed.

\subsubsection{Inhibitions of PKC, CKII and GSK3 $\beta$ did not affect Sph1 protein expression}

Protein kinases are key modulators of important signaling pathways, and protein phosphorylation regulates many cellular activities including gene expression [392]. Hence, prior to investigating the effects of PKC, CKII and GSK3 $\beta$ inhibitions on Sph1 aggrephagy behavior, it is critical to establish whether these kinases affect Sph1 protein expression. This analysis ensured that any effect observed with the kinase inhibitors in the downstream aggrephagy experiments is not due to changes in Sph1 protein expression.

SY5Y cells transiently overexpressing GFP-Sph1 were treated with the respective kinase inhibitors, and the levels of Sph1 under each condition were analyzed (Fig. 3.7). No observable changes in Sph1 protein levels were detected upon inhibitions of PKC, CKII and GSK3 $\beta$ (Fig. 3.7). This result indicates that the inhibitor concentrations used were suitable to study Sph1 aggrephagy behavior without altering the expression of Sph1. 


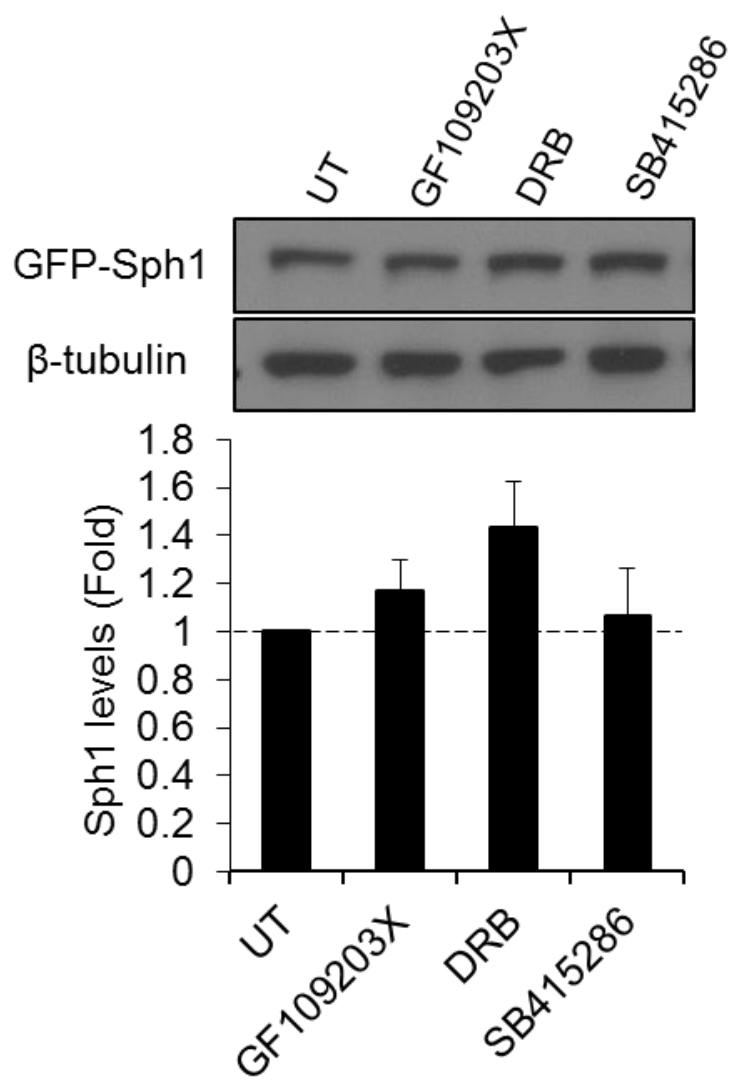

Figure 3.7 Inhibitions of PKC, CKII and GSK3 $\beta$ did not alter Sph1 protein expression. Top: Immunoblots of GFP-Sph1 in SY5Y cells transiently transfected with GFP-Sph1 for $24 \mathrm{~h}$, followed by inhibitions of PKC, CKII and GSK3 $\beta$ with $1.0 \mu \mathrm{M}$

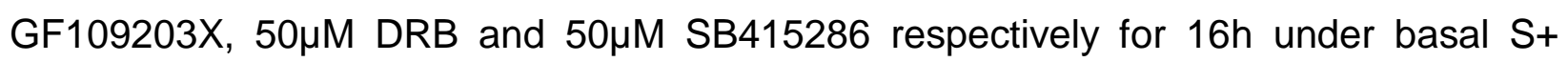
condition. Bottom: Quantification of the GFP-Sph1 protein levels under the different treatment conditions. All values are mean + S.E.M $(n=3)$, expressed as fold change against UT control. 


\subsubsection{PKC, CKII and GSK3 $\beta$ influence the levels of Agg and Agm formed by Sph1}

Before looking at the effects of PKC, CKII and GSK3 $\beta$ on the aggrephagy behavior of Sph1, the influence of these kinases on the aggregation propensity of Sph1 to form the Agg and Agm were first analyzed.

SY5Y cells transiently overexpressing GFP-Sph1 were treated with MG132, and the percentage of cells containing Agg and Agm were quantified in the absence or presence of kinase inhibitors. We observed that inhibitions of PKC and GSK3 $\beta$ led to a significant increase in the percentages of Agg from approximately $18 \%$ to $40 \%$ and $25 \%$ respectively (Fig. 3.8). In contrast, CKII inhibition reduced the Agg levels to approximately 8\% (Fig. 3.8). For the Agm, PKC inhibition did not alter the levels of Agm, whereas CKII and GSK3 $\beta$ inhibitions significantly reduced the levels from approximately $23 \%$ to $10 \%$ (Fig.3.8). The latter observations agree with the published data that CKII and GSK3 $\beta$ regulate the propensity of Sph1 to form Agm-like inclusions [201, 202].

Together, the results demonstrate that PKC, CKII and GSK3 $\beta$ modulates the levels of Agg and Agm formed by Sph1 under proteasomal stress. Additionally, since Agg are cleared constitutively by basal autophagy, the accumulation of Agg upon CKII and GSK3 $\beta$ inhibitions suggest a possible defect in Agg turnover. This implies a potential role of CKII and GSK3 $\beta$ in regulating basal aggrephagy. 

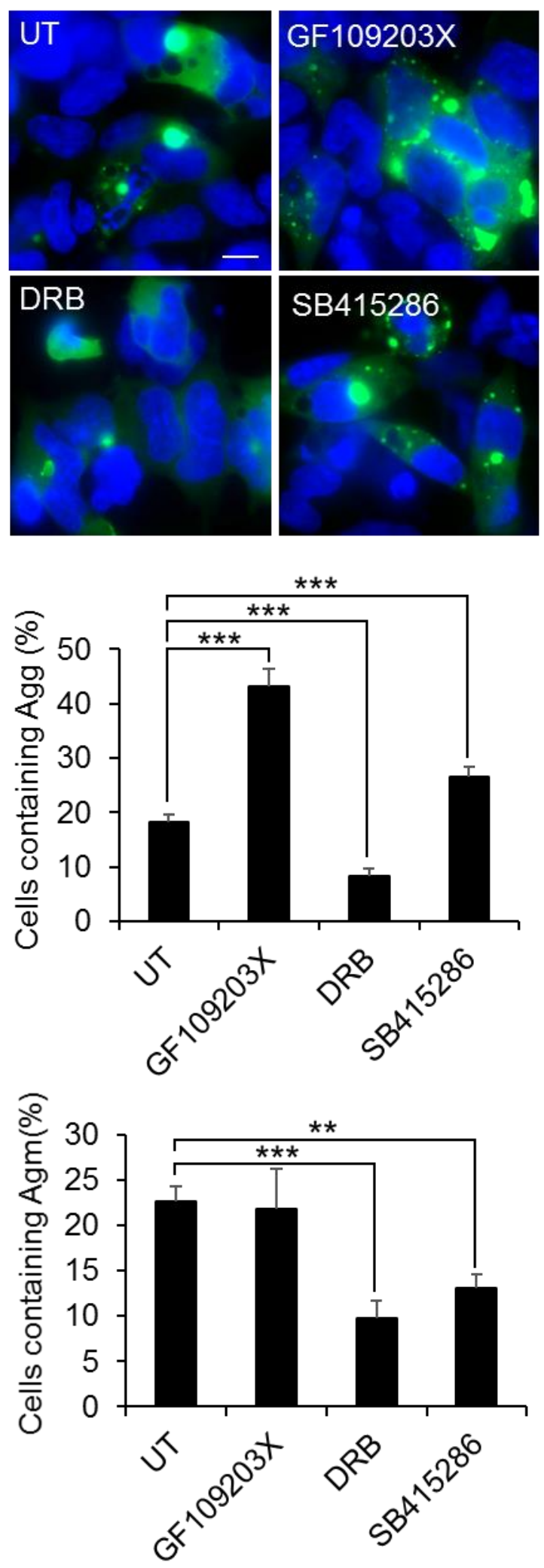
Figure 3.8 PKC, CKII and GSK3 $\beta$ influence the formation of Agg and Agm by Sph1. Top: Immunofluorescence images of the Agg and Agm formed by GFP-Sph1 in SY5Y cells transiently transfected with GFP-Sph1 for $24 \mathrm{~h}$, followed by $2 \mu \mathrm{M}$ MG132 proteasomal

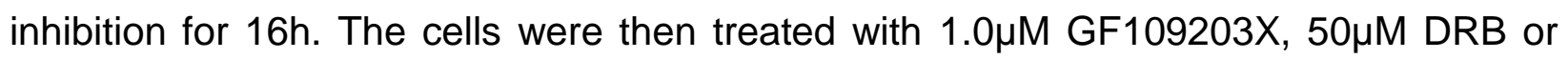
$50 \mu \mathrm{M}$ SB415286 for $12 \mathrm{~h}$. Bottom: Quantification of the percentage of cells containing Sph1 Agg and Agm under the different treatment conditions. At least 100 transfected cells from random fields were analyzed for each condition. Nuclei were stained with DAPI. All values are mean + S.E.M ( $n=3-12)$. Differences against untreated (UT) control are significant for ${ }^{* *} p<0.01,{ }^{* * *} p<0.005$. Scale bars, $10 \mu \mathrm{m}$. 


\subsubsection{Chemical inhibitions of PKC, CKII and GSK3 $\beta$ perturb clearance of Sph1 Agg by basal aggrephagy}

Agg formed by Sph1 is cleared by basal autophagy [18]. However, the underlying mechanism is independent of K63U on Sph1 [18]. In this study, we observed that Sph1 is phosphorylated by PKC, CKII and GSK3ß (Fig. 3.6) and all three kinases influence Sph1 Agg levels (Fig. 3.8), thus suggesting a role of phosphorylation in regulating basal aggrephagy. To prove this hypothesis, the effects of various kinase inhibitions on Sph1 basal aggrephagy were examined based on the established basal Agg aggrephagy experimental paradigm [18].

For this assay [18], GFP-Sph1 was transiently overexpressed in SY5Y cells in the presence of MG132 proteasomal inhibitor to induce Sph1 Agg and Agm inclusions. With this preformed pool of inclusions, the clearance of Agg could be monitored independent of its formation. To monitor the basal clearance of Sph1 Agg, the cells were maintained in either (1) serum-rich S+ media (basal condition where Agg are cleared constitutively) or (2) S+ condition in the presence of autophagy inhibitor 3-methyladenine (3-MA) (Fig. 3.9a). When basal aggrephagy is functional, blockage of autophagy with 3-MA will lead to accumulation of Agg as basal autophagic turnover of Agg is inhibited (Fig. 3.9a). On the other hand, if the basal autophagy pathway is non-functional, addition of 3-MA will not affect the Agg levels as the autophagy turnover is already perturbed (Fig. 3.9a). Indeed, 3-MA treatment led to $\sim 1-1.5$-folds increase in cells containing Agg basally as compared to the untreated cells (UT) across all the experimental sets (Fig. 3.9b-d; UT). These results affirm the constitutive removal of Sph1 Agg by basal aggrephagy in our study.

PKC inhibition. Addition of PKC inhibitor significantly enhanced the percentage of Aggcontaining cells compared to UT cells under basal $S_{+}$condition (Fig. 3.9b). This is consistent with the earlier observation that PKC inhibition increased Agg levels (Fig. 3.8). However, blockage of basal autophagy with 3-MA did not increase the percentage of cells containing Agg under PKC inhibition (Fig. 3.9b), suggesting that loss of PKC activity abrogated basal aggrephagy of Agg. Thus, the increase in Agg levels under PKC 
inhibition in S+ condition (Fig. 3.8 and 3.9b) was more likely a consequence of defective basal turnover, rather than enhanced propensity of Sph1 to form Agg.

CKII inhibition. As observed in Fig. 3.8, addition of CKII inhibitor significantly reduced the percentage of cells containing Agg compared to UT cells under basal S+ condition (Fig. 3.9c). Upon 3-MA autophagic inhibition, an approximately 1 -fold increase in Agg levels was observed under CKII inhibition (Fig. 3.9c), indicating productive basal aggrephagy flux was still ongoing under CKII inhibition. However, the Agg flux was significantly lowered by approximately 1 -fold compared to UT cells (Fig. 3.9c). This finding demonstrates that while basal autophagy is still preserved upon CKII inhibition, the rate of Agg turnover is much reduced.

GSK3 $\beta$ inhibition. As observed for PKC effects, GSK3 $\beta$ inhibition significantly increased the percentage of cells containing Agg under basal S+ condition (Fig. 3.8 and 3.9d). Further addition of 3-MA also did not result in significant accumulation of Agg containing cells compared to $S+$ condition under GSK3 $\beta$ inhibition (Fig. 3.9d). Hence, inhibition of GSK3 $\beta$ also perturbs basal aggrephagy, which likely have contributed to the enhanced Agg levels observed under S+ condition.

Collectively, our results demonstrate that PKC, CKII and GSK3 $\beta$ modulate the removal of Sph1 Agg by basal aggrephagy. While inhibitions of PKC and GSK3 $\beta$ completely abolished basal clearance of Agg, CKII only partially impaired the process. 
a

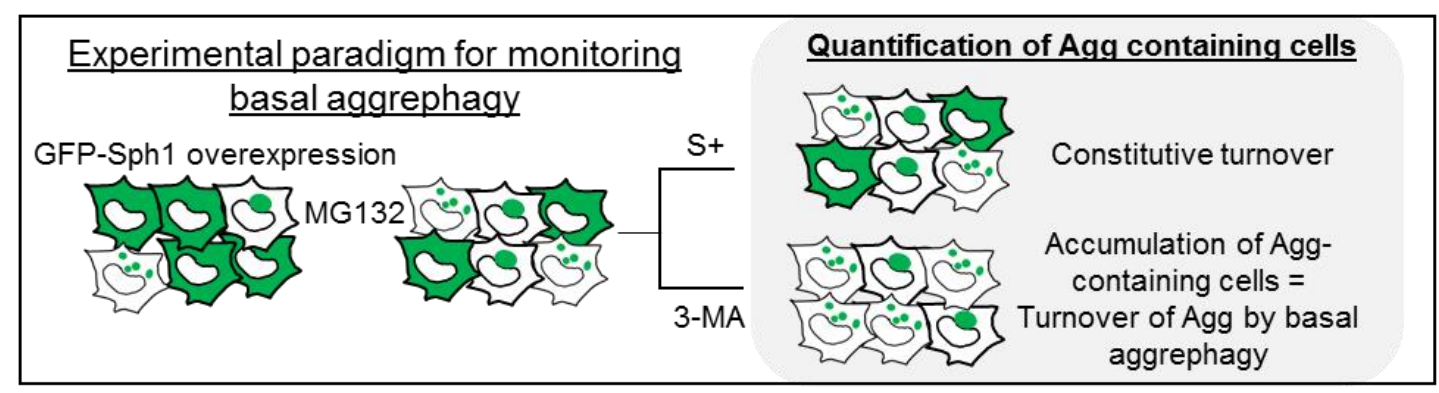

b

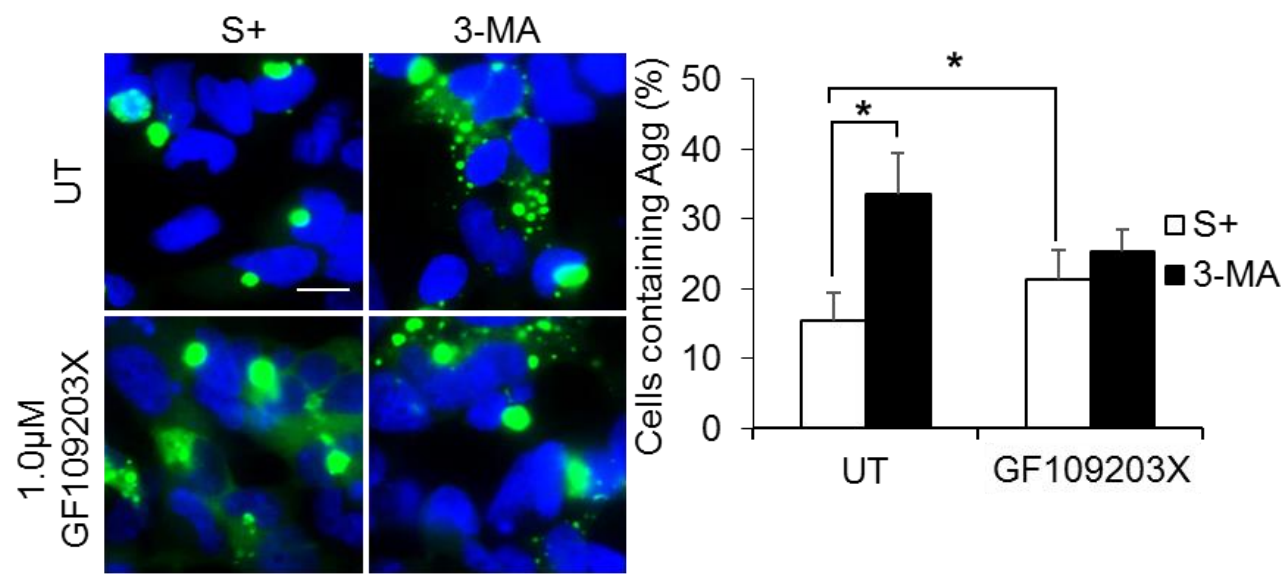

C

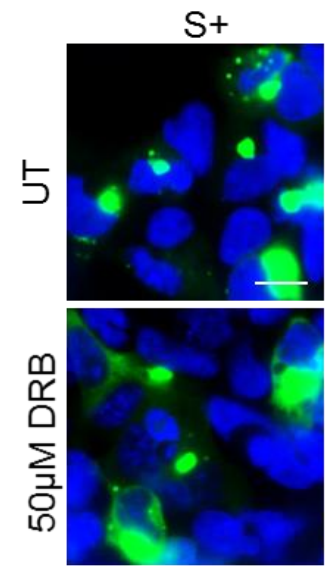

3-MA
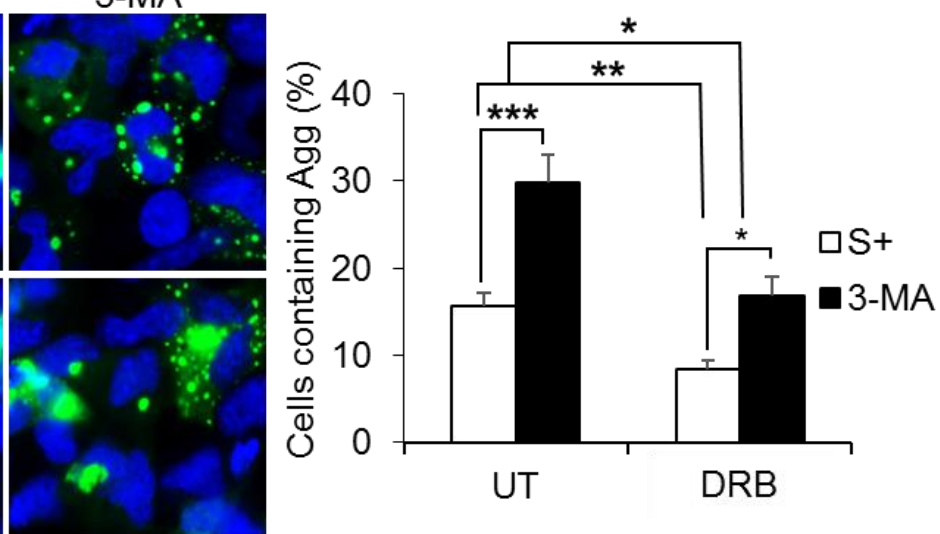

d
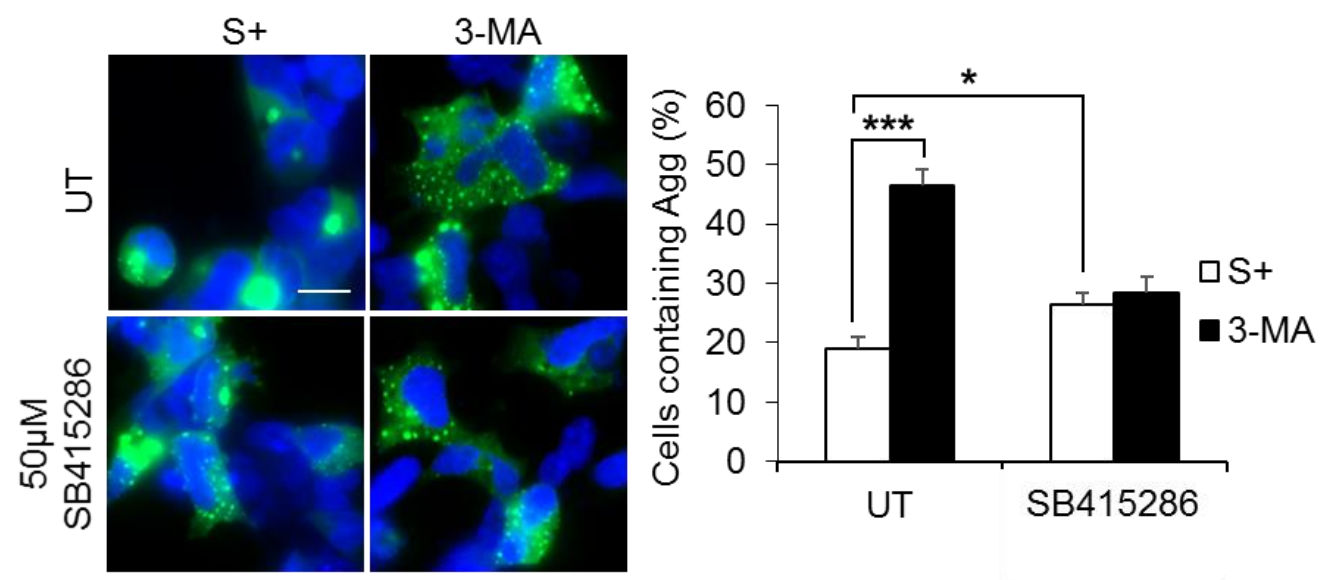
Figure 3.9 Inhibitions of PKC, CKII and GSK3 $\beta$ perturb clearance of Sph1 Agg by basal aggrephagy. (a) Illustration of the experimental paradigm used to investigate basal clearance of Sph1 Agg. (b-d) (Left) Immunofluorescence images of the Agg and Agm populations in SY5Y cells transiently overexpressing GFP-Sph1. The cells were treated with $2 \mu \mathrm{M}$ MG132 for $16 \mathrm{~h}$, followed by incubation in serum-rich $\mathrm{S}+$ media or $\mathrm{S}+$ with the addition of $10 \mathrm{mM}$ autophagy inhibitor 3-MA, in the absence (UT) or presence of (b) $1.0 \mu \mathrm{M}$

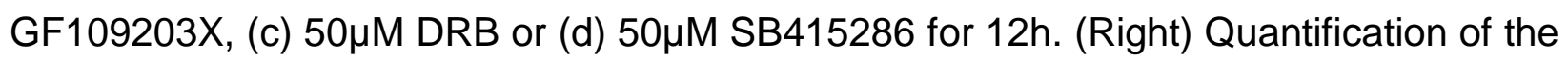
percentage of cells containing Sph1 Agm under the different treatment conditions. At least 100 transfected cells from random fields were analyzed for each condition. Nuclei were stained with DAPI. All values are mean + S.E.M $(n=3-10)$. Differences against UT or S+ are significant for ${ }^{*} p<0.05,{ }^{* * *} p<0.005$. Scale bars, $10 \mu \mathrm{m}$. 


\subsubsection{Genetic ablation of PKC, CKII and GSK3 $\beta$ perturb clearance of Sph1 Agg by basal aggrephagy}

To circumvent potential off-target effects of chemical inhibition of kinases, transient knockdown (KD) of PKCa, PKCY, CKII $\beta$ and GSK3 $\beta$ expression in SY5Y cells were carried out using siRNA technique to monitor the effects on Agg clearance. The knockdown efficiency of the various kinases was shown in Fig. 3.10a-d.

Similar to chemical inhibition, PKCa (Fig. 3.10a) and GSK3 $\beta$ (Fig. 3.10d) KD enhanced the population of cells containing Agg under basal S+ condition. Interestingly, PKCY (Fig. 3.10b) and CKII $\beta$ (Fig. 3.10c) KD had negligible effect on Agg levels. Based on the specific KD effects of each kinase isoform on Agg levels, the Agg changes due to chemical inhibition of pan PKC and CKII (Fig. 3.8 and 3.9b-c) were likely caused by PKC $\alpha$ isoform.

Next, the effects of various kinase KD on Sph1 aggrephagy behavior were tested. In the scrambled (scr) siRNA control cells across all experimental sets, autophagic inhibition with 3-MA significantly increased the percentage of cells harboring Agg by approximately 2-folds compared to the basal S+ condition (Fig. 3.10a-d; Scr). Thus, basal aggrephagy was functional in the scrambled siRNA control cells. In contrast, negligible accumulation of Agg was observed upon 3-MA inhibition in PKCa (Fig. 3.10a), PKCY (Fig. 3.10b) and GSK3 $\beta$ (Fig. 3.10d) KD cells. These results together with previous chemical inhibition data (Fig. 3.9) affirmed that loss of PKC and GSK3 $\beta$ activities perturbed basal turnover of Sph1 Agg. In the case of PKC, the $\alpha$ and $y$ isoforms modulate Sph1 basal aggrephagy.

Unlike the reduced Agg flux seen with CKII chemical inhibition (Fig. 3.9c), significant unperturbed Agg flux was still observed in CKII $\beta$ KD cells under autophagic inhibition with 3-MA (Fig. 3.10c). This result shows that CKII $\beta$ is not the key isoform for modulating Agg clearance. 
Together, the examination of basal aggrephagy behaviors of Sph1 Agg under kinase chemical inhibition and KD demonstrate that $\mathrm{PKC} \alpha, \mathrm{PKC} \gamma$ and $\mathrm{GSK} 3 \beta$ are regulators of Sph1 Agg clearance. CKII on the other hand, appears to partially modulate basal aggrephagy. The $\alpha$ instead of $\beta$ isoform may be more important in CKII regulation of Agg clearance. 
a

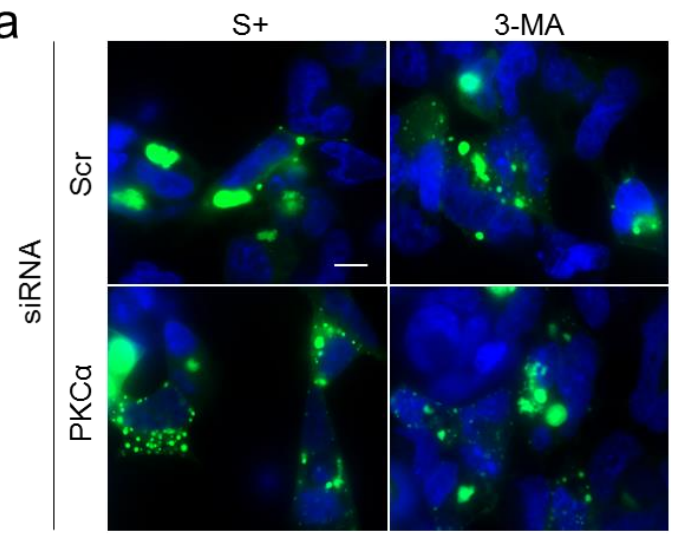

b

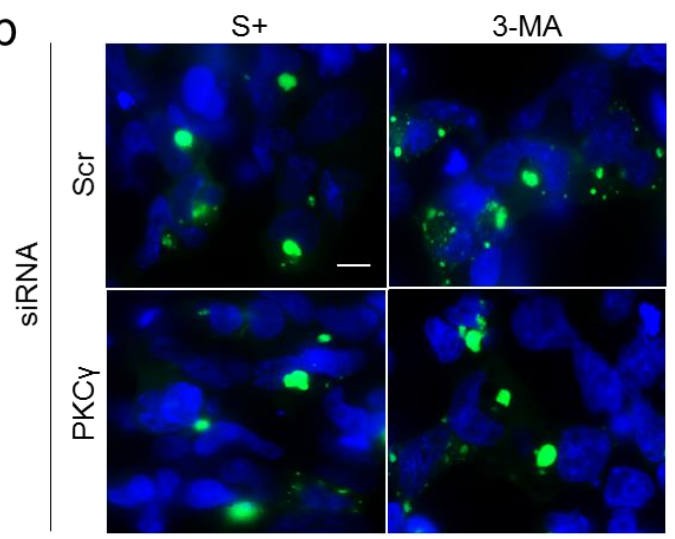

C

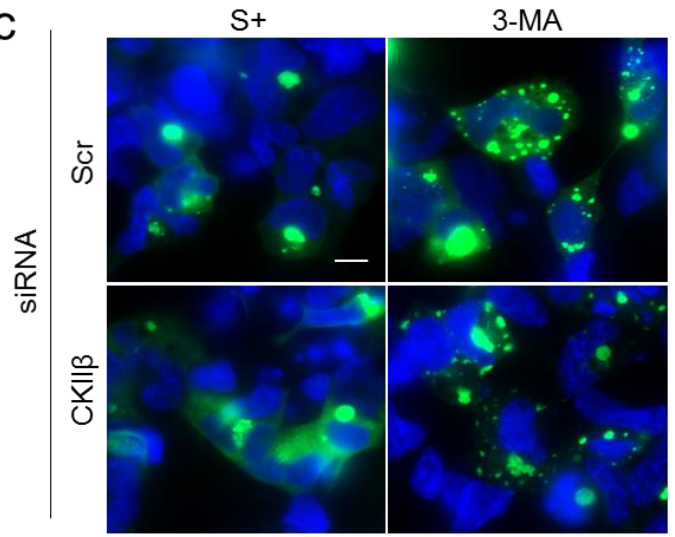

d

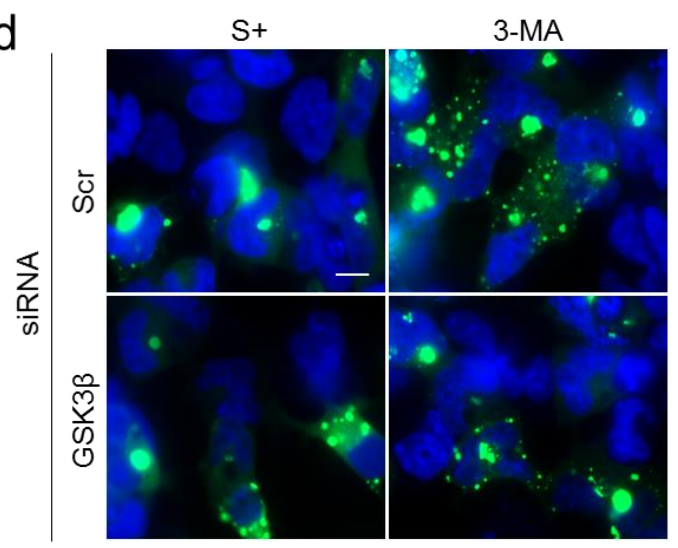

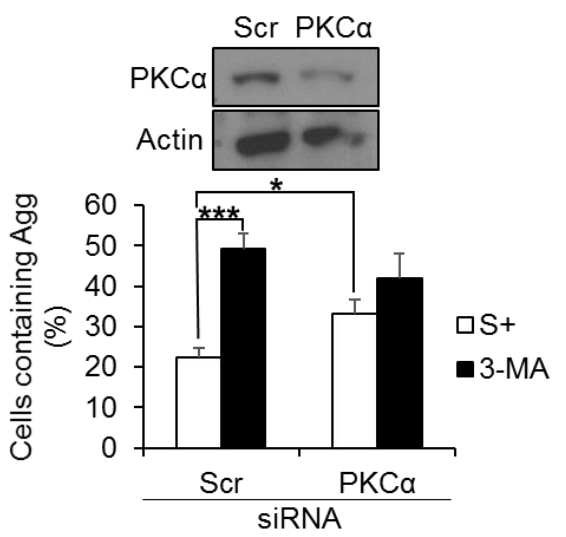
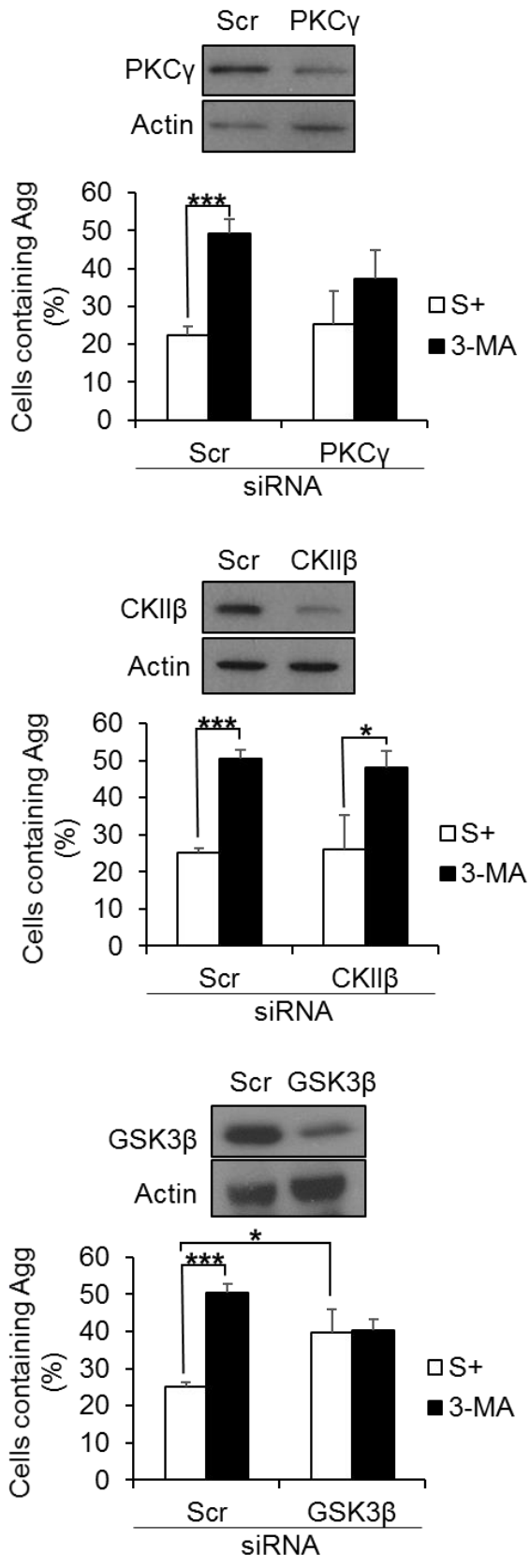
Figure 3.10 siRNA-mediated KD of PKC $\alpha$, PKCY and GSK3 $\beta$ perturb clearance of Sph1 Agg by basal aggrephagy. (Left) Immunofluorescence images of GFP-Sph1 Agg and Agm populations in scrambled siRNA control and (a) PKCa, (b) PKCY, (c) CKII $\beta$ and (d) GSK3 $\beta$ knockdown (KD) SY5Y cells. The cells were transiently transfected with the respective siRNAs for $24 \mathrm{~h}$, followed by treatment with $2 \mu \mathrm{M}$ proteasomal inhibitor MG132 for $16 \mathrm{~h}$. The cells were then incubated in serum-rich $\mathrm{S}+$ media, or $\mathrm{S}+$ in the presence of 10mM 3-MA for 12h. (Right, top) Immunoblots of endogenous (a) PKCa, (b) PKCy, (c) CKII $\beta$ and (d) GSK3 $\beta$ in the scrambled control and KD cells. (Right, bottom) Quantification of the percentage of cells containing Sph1 Agg under the different treatment conditions. At least 100 transfected cells from random fields were analyzed for each condition. Nuclei were stained with DAPI. All values are mean + S.E.M $(n=3-6)$. Differences against $S+$ or scrambled control are significant for ${ }^{*} p<0.05,{ }^{* * *} p<0.005$. Scale bars, $10 \mu \mathrm{m}$. 


\subsubsection{Chemical inhibitions of PKC, CKII and GSK3 $\beta$ perturb clearance of Sph1 Agm by inducible aggrephagy}

Crosstalk between phosphorylation and ubiquitination has been implicated in the regulation of protein degradation [388]. The evidence for interplay between phosphorylation and ubiquitination has propelled us to also investigate the role of phosphorylation in regulating Sph1 K63U-dependent inducible aggrephagy process.

Similar to basal aggrephagy, inducible aggrephagy phenomenon was studied based on a previously described assay (Fig. 3.11a) [18]. In this paradigm, SY5Y cells transiently overexpressing GFP-Sph1 were treated with proteasomal inhibitor MG132 to induce Agm formation. With this preformed Agm pool, the clearance of Agm under starvation induced autophagic condition was monitored. In a cellular environment with intact inducible aggrephagy, starvation stimulates the removal of Agm and causes a reduction in the proportion of cells harboring the Agm (Fig. 3.11a). Conversely, no reduction in Agm pool upon starvation is observed when inducible aggrephagy is impaired (Fig. 3.11a).

In agreement with earlier observation (Fig. 3.8) as well as published reports [201, 202], inhibitions of CKII and GSK3 $\beta$ reduced the percentage of cells containing Agm (Fig. 3.11c and d). On the other hand, PKC inhibition did not alter the Agm levels (Fig. 3.11b). With this, we went on to examine the effect of starvation (S-) on the Agm pool. In the absence of kinase inhibitions (UT), S- significantly reduced the percentage of Agm containing cells from $\sim 25-30 \%$ to $\sim 15 \%$ across all the experimental sets (Fig. 3.11b-c; UT). This shows that starvation is an appropriate stimulus to induce Agm clearance by autophagy.

Next, we investigated the effects of kinase inhibitions on starvation-induced Agm clearance. Upon PKC, CKII and GSK3 $\beta$ inhibitions, S- did not reduce the percentage of cells harboring Agm as compared to $S_{+}$condition (Fig. 3.11b-d). These results demonstrate that loss of PKC, CKII and GSK3 $\beta$ functions interfere with Sph1 Agm turnover by inducible aggrephagy, thus suggesting that these three kinases also play a role in modulating clearance of Agm. 
a

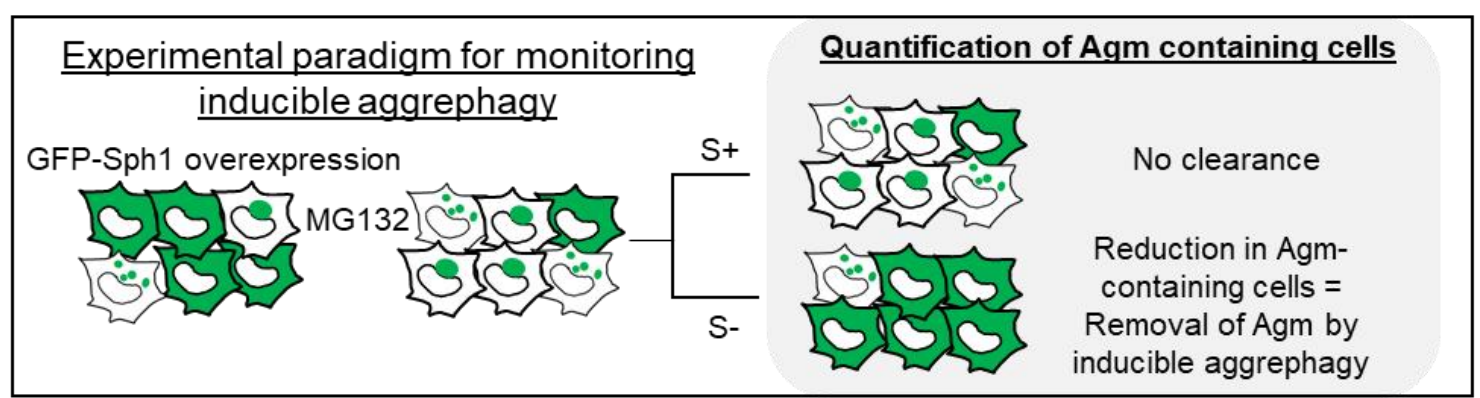

b

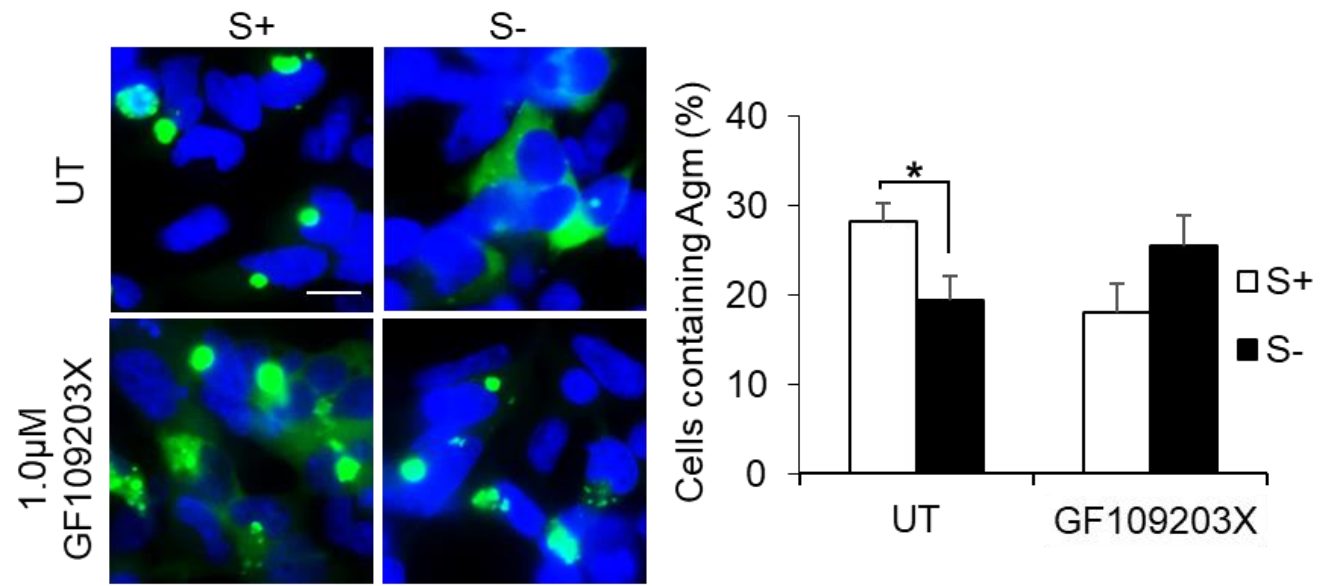

C

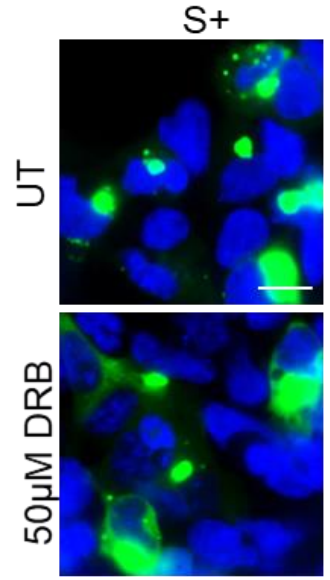

S-
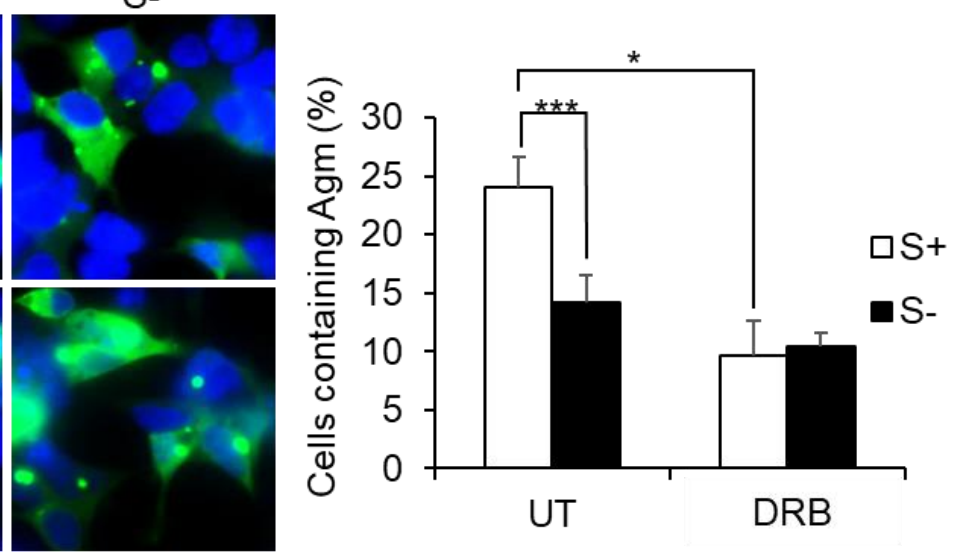

d

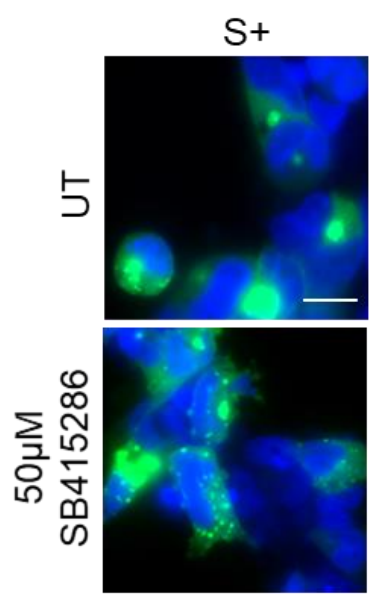

S-
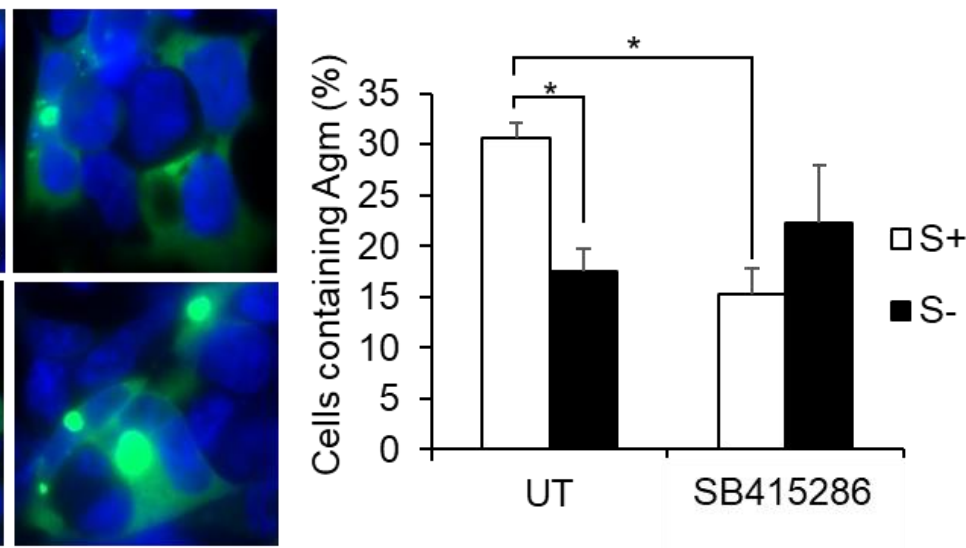
Figure 3.11 PKC, CKII and GSK3 $\beta$ inhibitions perturb clearance of Sph1 Agm by starvation-induced aggrephagy. (a) Illustration of the experimental paradigm used to investigate inducible clearance of Sph1 Agm. (b-d) (Left) Immunofluorescence images of the Agg and Agm populations in SY5Y cells transiently overexpressing GFP-Sph1. The cells were treated with $2 \mu \mathrm{M}$ MG132 for $16 \mathrm{~h}$, followed by incubation in serum-rich S+ or

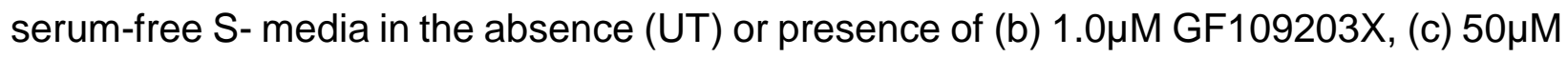
DRB or (d) $50 \mu \mathrm{M}$ SB415286 for 12h. (Right) Quantification of percentage of cells containing Sph1 Agm under the different treatment conditions. At least 100 transfected cells from random fields were analyzed for each condition. Nuclei were stained with DAPI. All values are mean + S.E.M $(n=3-10)$. Differences against UT or $S+$ are significant for ${ }^{*} p<0.05,{ }^{* * *} p<0.005$. Scale bars, $10 \mu \mathrm{m}$. 


\subsubsection{Genetic ablation of PKC, CKII and GSK3 $\beta$ perturb clearance of Sph1 Agm by inducible aggrephagy}

Here, the effects of PKC $\alpha, P K C \gamma, C K I I \beta$ and GSK3 $\beta$ KD on Sph1 inducible aggrephagy were investigated. In agreement with CKII and GSK3 $\beta$ inhibitions (Fig. 3.8 and Fig. 3.11 C-d) and published data [201, 202], KD of CKII $\beta$ and GSK3 $\beta$ reduced the percentage of cells containing Agm from approximately $37 \%$ to $30 \%$ and $20 \%$ respectively (Fig. 3.12c and d). This data once again highlights that CKII and GSK3 $\beta$ modulate the formation of Sph1 Agm.

Next, we assessed the effects of kinase KD on Agm clearance. Examination of the Agm population in the scrambled (Scr) control cells across all the experimental sets revealed that starvation (S-) reduced the proportion of cells harboring the Agm from approximately $40 \%$ to $30 \%$ (Fig. 3.12a-d; UT). Hence, starvation mediated inducible aggrephagy of Agm was functional in the scrambled siRNA control cells. However, in respective PKCa, PKCY, CKII $\beta$ and GSK3 $\beta$ KD cells, S- failed to reduce the percentage of cells harboring Agm as compared to S+ condition (Fig. 3.12a-d), indicating a defect in Sph1 Agm turnover by starvation mediated inducible autophagy

Together, both chemical inhibition and KD studies highlight that PKC, CKII and GSK3 $\beta$ participate in the clearance of Sph1 Agm by inducible aggrephagy. The involvement of phosphorylation in inducible aggrephagy further suggests that phosphorylation and ubiquitination may cooperate to determine the clearance of Agm. 

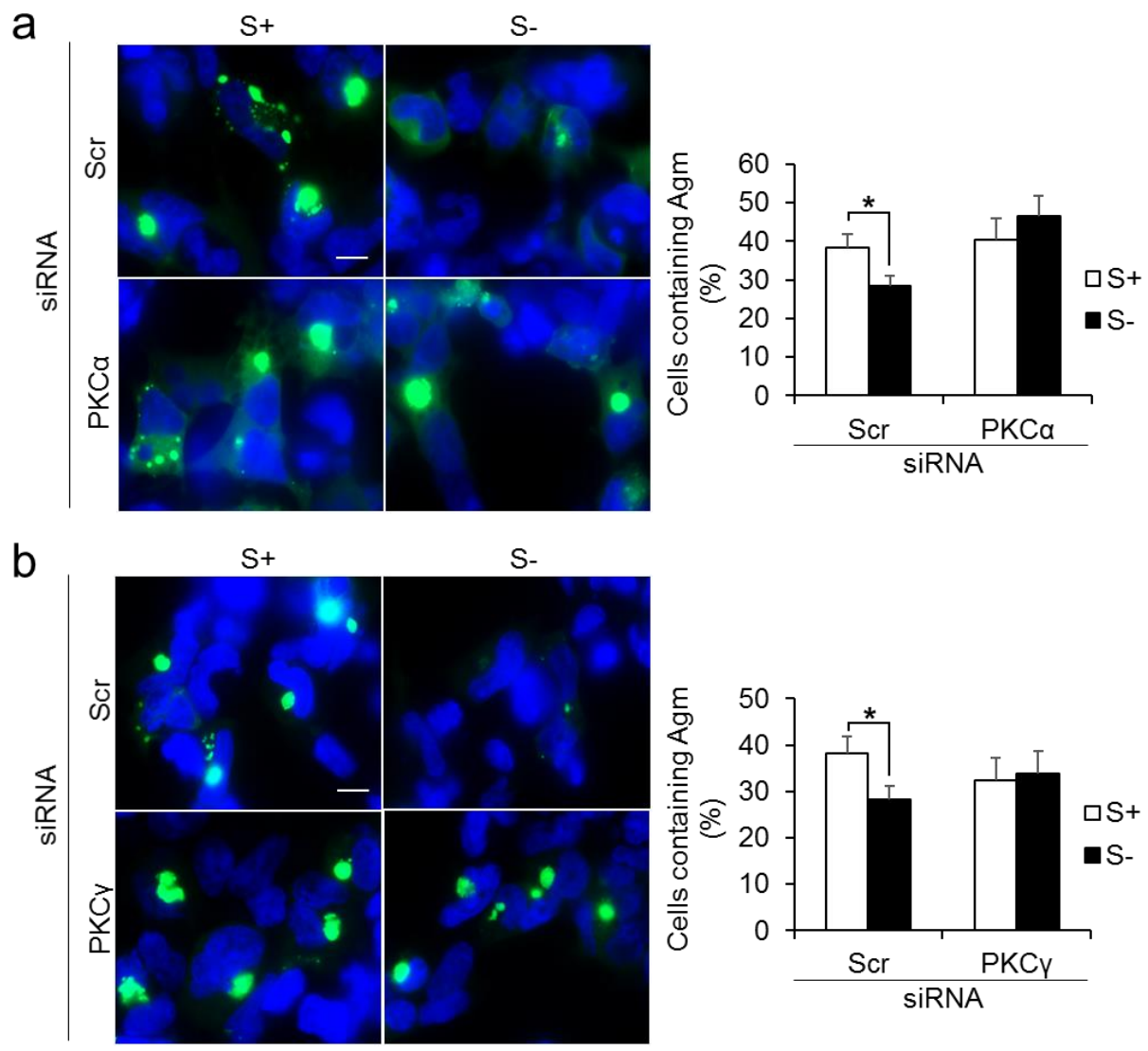

C

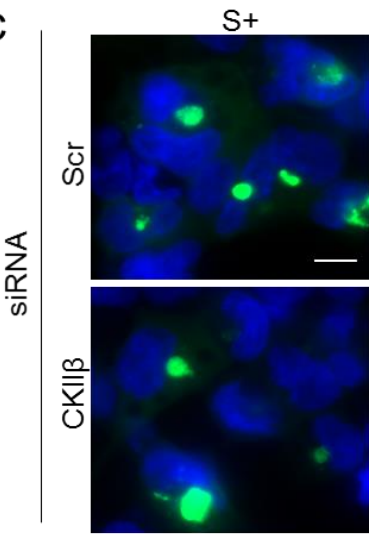

S-
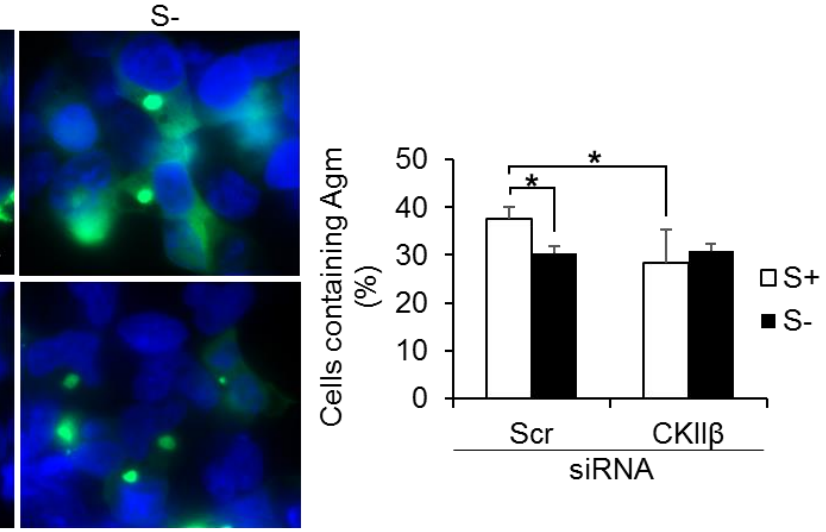

d

S+

S-
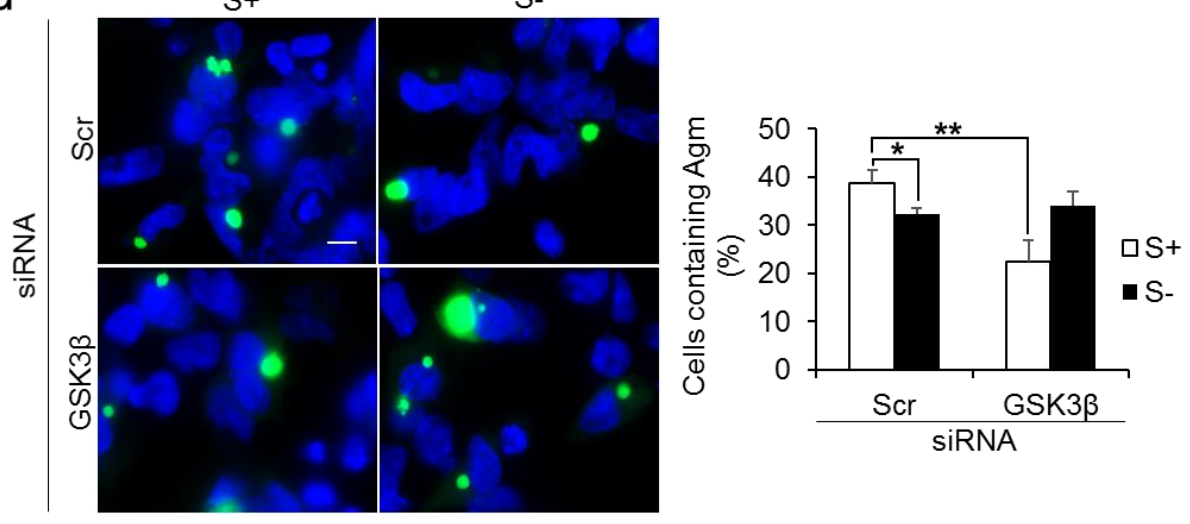
Figure 3.12 siRNA-mediated KD of PKC $\alpha, P K C Y$ and GSK3 $\beta$ perturb clearance of Sph1 Agm by starvation-induced aggrephagy. (Left) Immunofluorescence images of GFP-Sph1 Agg and Agm populations in scrambled siRNA control and (a) PKCa, (b) PKCY, (c) CKII $\beta$ and (d) GSK3 $\beta$ knockdown (KD) SY5Y cells. The cells were transiently transfected with the respective siRNAs for $24 \mathrm{~h}$, followed by treatment with $2 \mu \mathrm{M}$ proteasomal inhibitor MG132 for $16 \mathrm{~h}$. The cells were then incubated in serum-rich S+ media or serum-deprived S- media for 12h. (Right) Quantification of the percentage of cells containing Sph1 Agm under the different treatment conditions. At least 100 transfected cells from random fields were analyzed for each condition. Nuclei were stained with DAPI. All values are mean + S.E.M $(n=3-6)$. Differences against $S+$ or scrambled control are significant for ${ }^{*} p<0.05,{ }^{*} p<0.01$. Scale bars, $10 \mu \mathrm{m}$. 


\subsection{MODULATION OF SYNPHILIN-1 AGGREPHAGY BEHAVIOR UNDER KINASE INHIBITIONS IS NOT A CONSEQUENCE OF THE IMPAIRMENT OF THE GENERAL AUTOPHAGY PATHWAY}

The activities of PKC, CKII and GSK3 $\beta$ have been implicated in the regulation of autophagy cascade [370]. It is thus, important to validate whether the observed perturbations in basal and inducible aggrephagy under kinase inhibitions are specific to Sph1 turnover, or a result of general autophagy pathway failure. To address this, the LC3II flux in SY5Y cells under proteasomal stress in the absence and presence of PKC, CKII and GSK3 $\beta$ inhibitors were compared.

LC3 exists as two different forms. The cytosolic LC3 is represented by the form I (LC3-I), while the autophagosome membrane bound LC3 represents the form II (LC3-II). By measuring the extent of LC3-II accumulation in the presence of autophagic inhibitors like ammonium chloride and leupeptin, one can monitor the rate of LC3-II turnover, which also represents the autophagic activity in the cell. Higher accumulation of LC3-II under autophagic inhibition indicates higher rate of lysosomal degradation.

LC3 appears as a doublet in immunoblotting, where the slower migrating band (top band) corresponds to LC3-I and the faster migrating band (bottom band) to LC3-II. In SY5Y cells (UT), autophagic inhibition with lysosomal inhibitors ammonium chloride and leupeptin (NL) led to a significant accumulation of LC3-II (Fig. 3.13). This accumulation represents the pool of autophagosomes turnover by autophagy, and indicates the autophagy flux in SY5Y cells. Under PKC, CKII and GSK3 $\beta$ inhibitions, comparable levels of LC3-II flux were also observed (Fig. 3.13). These results attest that general autophagy activity is not affected by the various kinase inhibitions. Thus, the perturbation in Sph1 basal and inducible aggrephagy observed with kinase inhibitions (Fig. 3.9 and 3.11) was specific to Sph1 turnover rather than a consequence of general impairment in cellular autophagic activity. 


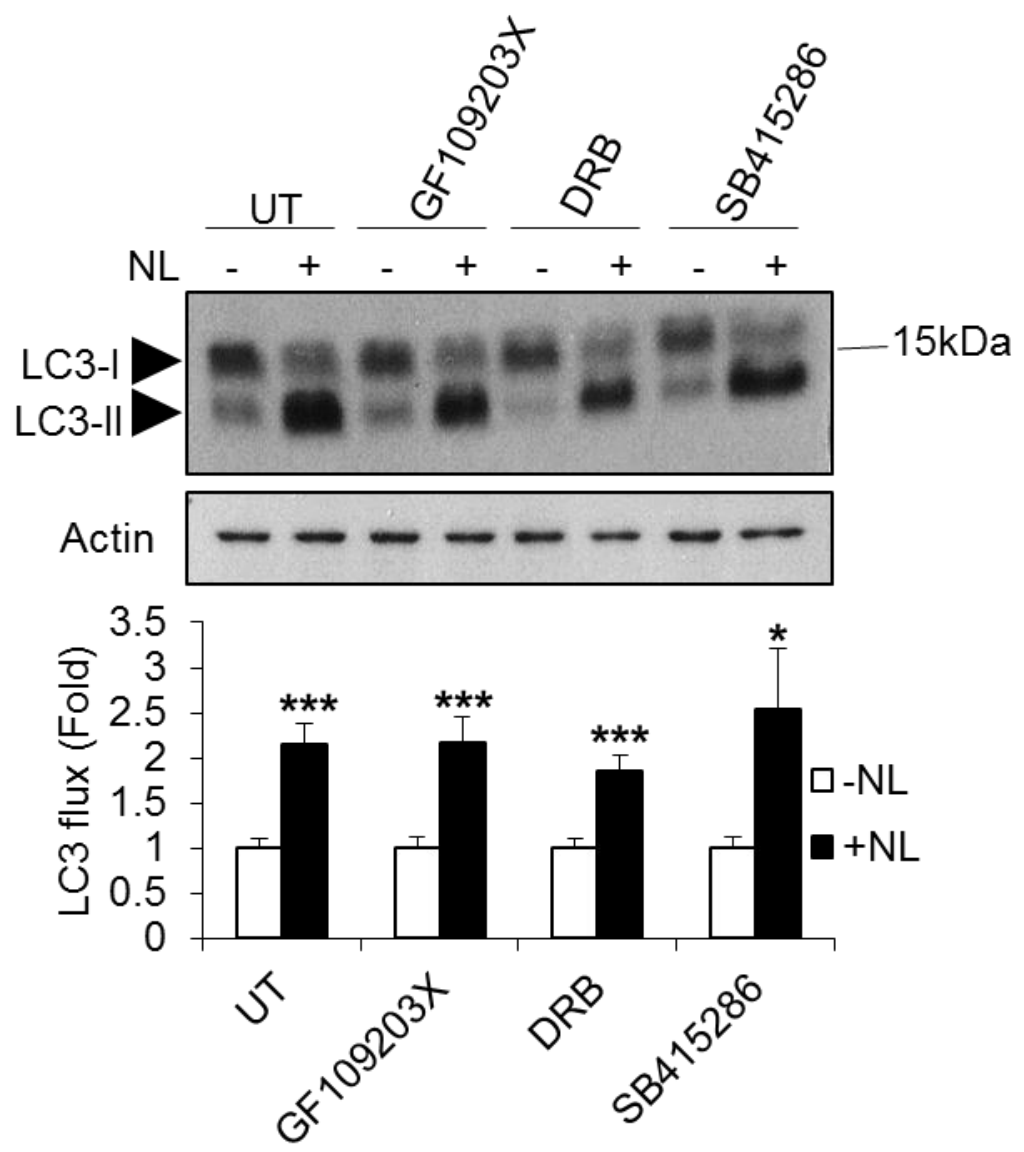

Figure 3.13 PKC, CKII and GSK3 $\beta$ inhibitions did not influence the cellular autophagic activity. Top: Immunoblot of LC3 in SY5Y cells treated with $2 \mu \mathrm{M}$

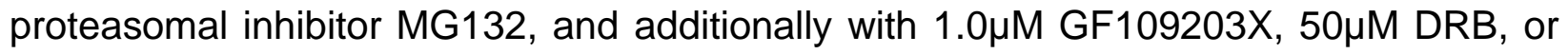
$50 \mu \mathrm{M}$ SB415286 for $16 \mathrm{~h}$. To examine the LC3-II flux, the cells were further treated in the absence or presence of lysosomal inhibitors NL (NL: $20 \mathrm{mM}$ ammonium chloride and $100 \mu \mathrm{M}$ leupeptin) for $4 \mathrm{~h}$. Bottom: Graph shows LC3-II flux calculated as fold increase in LC3-II levels upon lysosomal inhibition over LC3-II levels in the absence of NL. Values were expressed as mean + S.E.M ( $n=3)$. Differences against $-\mathrm{NL}$ are significant for ${ }^{* * *} \mathrm{p} \leq 0.005$. 


\subsection{GSK3ß MODULATES K63U ON SYNPHILIN-1}

With the observation that phosphorylation by PKC, CKII and GSK3 $\beta$ also regulates clearance of Sph1 Agm, we investigated the possibility of crosstalk between phosphorylation and ubiquitination as a mechanism to regulate inducible aggrephagy.

To explore the potential interplay between both PTM, we first examined how kinase inhibitions influence the ubiquitination levels on Sph1, particularly the K63U linkages that are important for facilitating inducible aggrephagy [18]. Briefly, SY5Y cells were transiently co-transfected with GFP-Sph1 and HA-Ub (WT ubiquitin) or HA-K63U. The cells were then treated with proteasomal inhibitor MG132 and the respective kinase inhibitors. Sph1 was subsequently immunoprecipitated with GFP-TRAP agarose beads, and immunoblotted for anti-HA (ubiquitin levels). In this assay, the cells were lysed under highly denaturing condition (RIPA with 6M urea lysis buffer) to extract both the soluble and insoluble (aggregated) forms of Sph1. This allows the examination of ubiquitination levels on the insoluble Sph1 fraction.

Based on the immunoprecipitation data, approximately the same levels of total ubiquitination ( $\mathrm{HA}-\mathrm{Ub}$ ), encompassing all possible ubiquitin linkages, were detected on the immunoprecipitated Sph1 under untreated (UT) as well as PKC, CKII or GSK3 $\beta$ inhibition conditions (Fig. 3.14a). While inhibitions of the various kinases did not alter the total ubiquitination levels on Sph1, analysis of the relative abundance of specific K63U linkage on Sph1 revealed that GSK3 $\beta$ inhibition significantly reduced the levels of K63U on Sph1 by approximately 0.3 -fold (Fig. 3.14b). These results suggest that phosphorylation of Sph1 influences the ubiquitin chain topologies rather than the abundance of total ubiquitination on Sph1. In particular, GSK3 $\beta$ is the key kinase that determines the levels of K63U on Sph1. This finding also suggests that the loss of K63U on Sph1 may underscore the reason behind impaired inducible aggrephagy of Agm observed in earlier GSK3 $\beta$ inhibition and KD experiments (Fig. 3.11d and 3.12d). The data here thus provides the first piece of evidence for a potential interplay between phosphorylation and ubiquitination in regulating Sph1 inducible aggrephagy. 
a
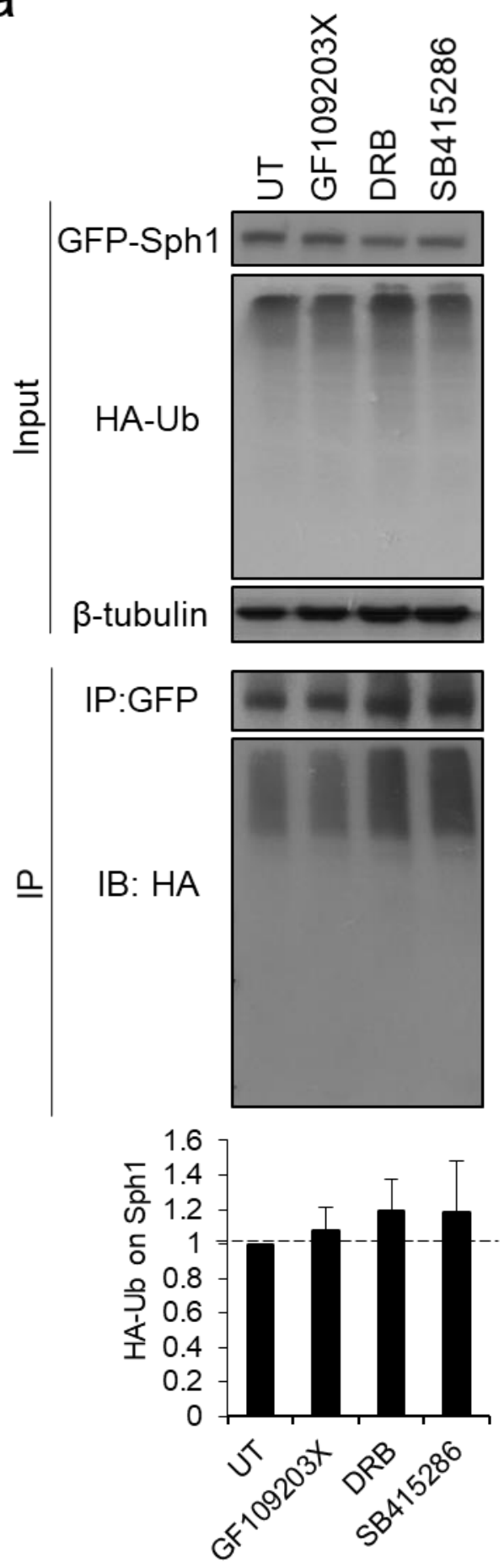

b
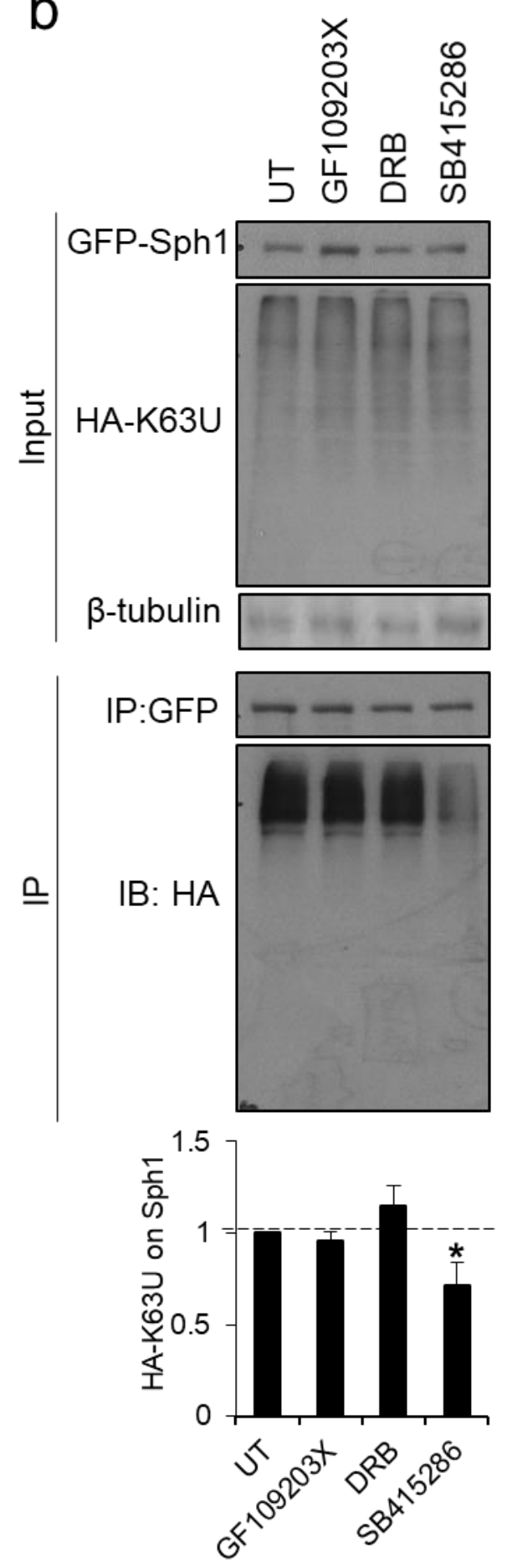


\section{Figure 3.14 Inhibition of GSK3 $\beta$ reduces K63U linkages on Sph1 under proteasomal}

stress. SY5Y cells were transiently co-transfected with GFP-Sph1 and (a) HA-Ub (WT $\mathrm{Ub}$ ) or (b) HA-K63U for $24 \mathrm{~h}$. The cells were then treated for $2 \mu \mathrm{M} \mathrm{MG132}$ proteasomal inhibitor for $16 \mathrm{~h}$, followed by treatment with $1.0 \mu \mathrm{M}$ GF109203X, 50 $\mu \mathrm{M}$ DRB or $50 \mu \mathrm{M}$ SB415286 for another $12 \mathrm{~h}$. The cells were lysed in RIPA buffer with addition of $6 \mathrm{M}$ urea, and the lysates were subjected to immunoprecipitation (IP) with GFP-TRAP agarose beads, followed by immunoblotting (IB) with anti-HA antibody. (Top) The upper panel (input) shows the total expression of GFP-Sph1 and HA-Ub or HA-K63U in the lysate. The lower panel (IP) shows the levels of GFP-Sph1 immunoprecipitated, and the levels of ubiquitination on GFP-Sph1. (Bottom) Quantification of HA-Ub and HA-K63U levels on Sph1. Values were expressed as mean + S.E.M $(n=3)$. Differences against UT control are significant for ${ }^{*} \mathrm{p}<0.05$. 


\subsection{GSK3 $\beta$ INTERACTS WITH PARKIN AND MODULATES SYNPHILIN-1 AND PARKIN INTERACTION}

\subsubsection{Background: Parkin as an E3 ligase that modulates K63U on Sph1 and inducible aggrephagy}

Ubiquitination of proteins involves the concerted action of the E1 ubiquitin-activating enzyme, E2 ubiquitin-conjugating enzymes and E3 ubiquitin-protein ligases [393]. In this cascade, the E3 ligase functions as a scaffold that binds to the substrate protein and specific E2, to mediate the transfer of ubiquitin molecule from E2 to the substrates [393]. The E3 ligase can also facilitate the addition of new ubiquitin molecule to the lysine residue of an existing ubiquitin to form polyubiquitin chain [394].

Sph1 is known to interact with several E3 ligases, including Dorfin [196], SIAH [197, 202] and Parkin [198]. Among these, Parkin was shown to interact with Sph1, and mediate K63U on Sph1 to promote formation and clearance of Sph1 inclusions [198, 200]. These findings pointed us to explore Parkin as the E3 ligase that may interface with GSK3 $\beta$, also shown to influence K63U levels on Sph1 (Fig. 3.14b), to direct K63U of Sph1 to facilitate inducible aggrephagy in our study.

To investigate this hypothesis, we first assayed whether GSK3 $\beta$ interacts with Parkin via co-IP. Although PKC and CKII inhibitions did not alter the K63U levels on Sph1, we did not rule out the possibility that both kinases could influence Parkin activity to modulate Sph1 inducible aggrephagy behavior. Thus, the interactions of both kinases with Parkin were also examined.

\subsubsection{PKC $\alpha$ and GSK3 $\beta$ interact with Parkin under proteasomal stress}

The interactions between Parkin and the kinases were assayed via co-IP. Briefly, mCherry-Parkin SY5Y stable cells were transiently transfected with HA-PKCa, HA-PKCY, 
myc-CKII $\beta$ or HA-GSK3 $\beta$ under basal $S+$ condition in the absence and presence of proteasomal stress. Parkin was then immunoprecipitated with anti-mCherry antibody and immunoblot for the presence of HA- or myc-tagged kinase isoforms.

Based on the co-IP data, HA-PKCa co-immunoprecipitated with mCherry-Parkin under proteasomal inhibition with MG132, while negligible interaction was observed under basal S+ condition (Fig. 3.15a). The interaction between PKCa and Parkin mirrored what was observed earlier between PKCa and Sph1 (Fig. 3.4a), where the interaction was more apparent under proteasomal stress. On the other hand, HA-PKCY or myc-CKII $\beta$ was not detected with immunoprecipitated mCherry-Parkin under $S_{+}$or MG132 condition, highlighting that both kinases do not interact with Parkin (Fig. 3.15b and c).

Assessment for the interaction between mCherry-Parkin and HA-GSK3 $\beta$ revealed that the kinase only co-immunoprecipitated with Parkin under proteasomal inhibition with MG132 (Fig.3.15d). This interaction also mimicked what was observed earlier between Sph1 and GSK3 $\beta$ (Fig. 3.4d), where the interaction was also more apparent under proteasomal stress.

Collectively, the findings from the co-IP interaction study demonstrates that PKC $\alpha$ and GSK3 $\beta$ interact with Parkin in response to proteasomal stress. These enhanced interactions with both kinases under proteasomal stress was also observed between Sph1 and the two kinases (Fig. 3.4a and d). These results suggest a potential tripartite interaction between Sph1, Parkin and PKCa or GSK3 $\beta$ in response to proteasomal stress. We tested this hypothesis by examining whether kinase inhibitions perturb the interaction between Sph1 and Parkin in section 3.8.3. 

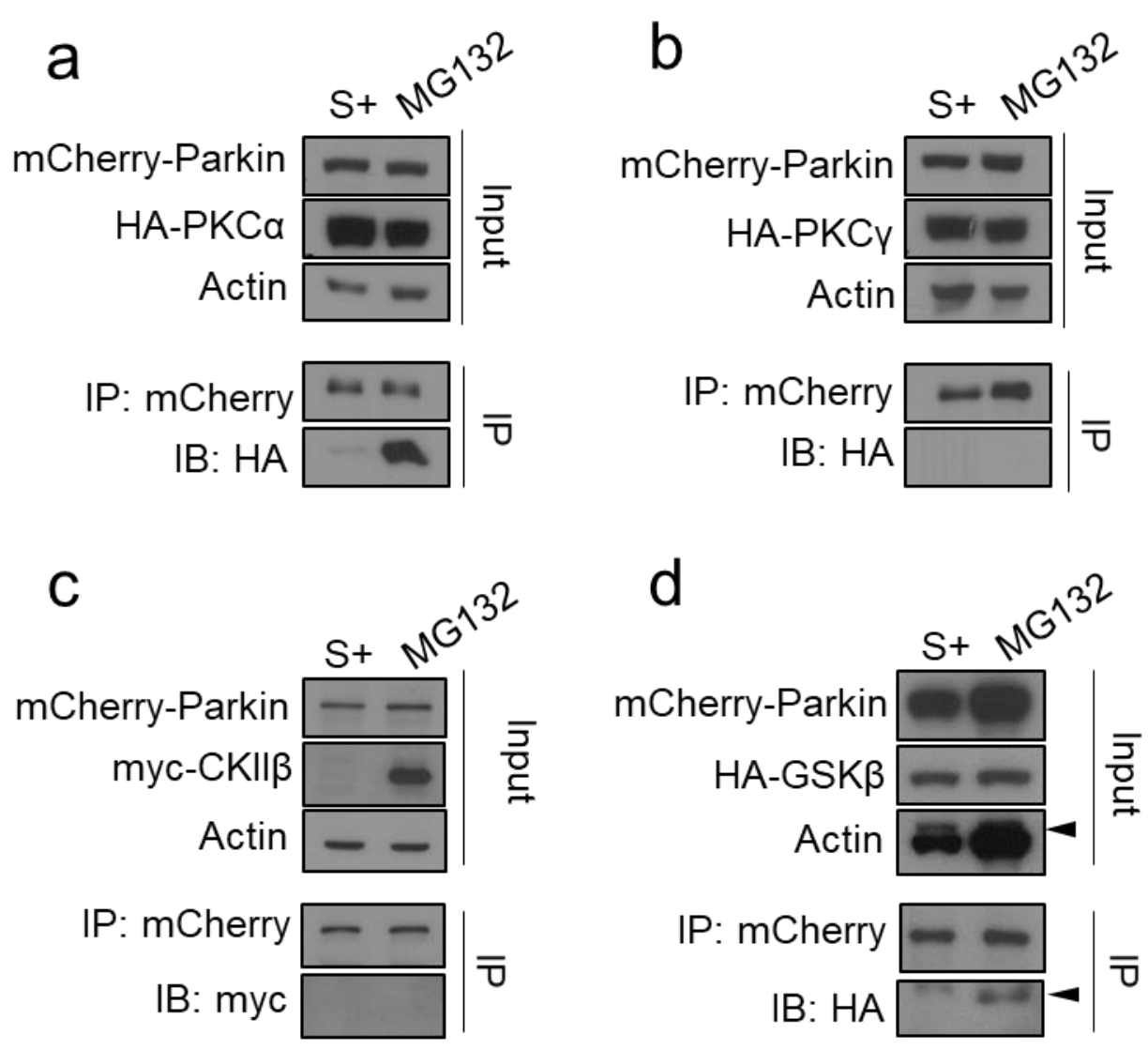

Figure 3.15 PKC $\alpha$ and GSK3 $\beta$ interact with Parkin in response to proteasomal stress. mCherry-Parkin SY5Y stable cells were transiently transfected with the individual kinase isoform represented in the figure by (a) HA-PKCa, (b) HA-PKCy, (c) myc-CKII $\beta$ and (d) HA-GSK3 $\beta$ for $24 \mathrm{~h}$. The media was then refreshed with serum-rich $\mathrm{S}+$ media, or with the addition of $2 \mu \mathrm{M}$ MG132 proteasomal inhibitor. Cells were lysed with $1 \%$ NP-40 lysis buffer, and the lysates were subjected to immunoprecipitation (IP) with anti-mCherry antibody, followed by immunoblotting (IB) with either anti-HA or anti-myc antibody. The upper panel (input) shows the total expression of mCherry-Parkin and the respective kinase isoform in the lysate. The lower panel (IP) shows the levels of mCherry-Parkin immunoprecipitated, and the levels of the individual kinase isoform that coimmunoprecipitated with mCherry-Parkin. The co-IP experiments were repeated 3 times $(n=3)$ 


\subsubsection{CKII and GSK3 $\beta$ regulate the interaction between Sph1 and Parkin under proteasomal stress}

mCherry-Parkin stable cells were transiently transfected with GFP-Sph1 and incubated under proteasomal inhibition with MG132. The effects of kinase inhibitions on Sph1Parkin interaction was examined by immunoprecipitating Parkin with anti-mCherry antibody and immunoblotting for GFP-Sph1.

First, an enhanced level of GFP-Sph1 was observed to co-immunoprecipitate with mCherry-Parkin upon proteasomal inhibition (Fig. 3.16, $n=2$ ). This data suggests that Sph1 interacts more with Parkin under proteasomal stress, which aligned with the reported data that Parkin plays an active role in regulating Sph1 behavior under proteasomal inhibition by promoting Sph1 Agm formation [199].

Next, we examined the effects of kinase inhibitions on the interaction between Sph1 and Parkin. Under PKC inhibition, the levels of GFP-Sph1 co-immunoprecipitated with Parkin were not altered as compared to the untreated (UT) cells under proteasomal inhibition (Fig. 3.16). This suggests that PKC does not modulate the interaction between Sph1 and Parkin. On the other hand, although CKII $\beta$ did not interact with Sph1 or Parkin in our study, inhibition of CKII significantly reduced the levels of GFP-Sph1 coimmunoprecipitated with mCherry-Parkin (Fig. 3.16). Similarly, under GSK3 $\beta$ inhibition, a reduction in the levels of co-immunoprecipitated GFP-Sph1 with mCherry-Parkin was also observed (Fig. 3.16).

These results suggest that CKII and GSK3 $\beta$ modulate the interaction between Sph1 and Parkin. Specifically, GSK3 $\beta$ may act in complex with Sph1 and Parkin to potentially modulate Sph1 inducible aggrephagy behavior. We postulated that complex formation between Sph1-Parkin-GSK3 $\beta$ is induced upon proteasomal stress, whereby GSK3 $\beta$ modulates the interaction between Sph1 and Parkin and thereby, regulating the levels of K63U linkages on Sph1. The latter effect will subsequently affect the clearance propensity of the Agm by inducible aggrephagy. 

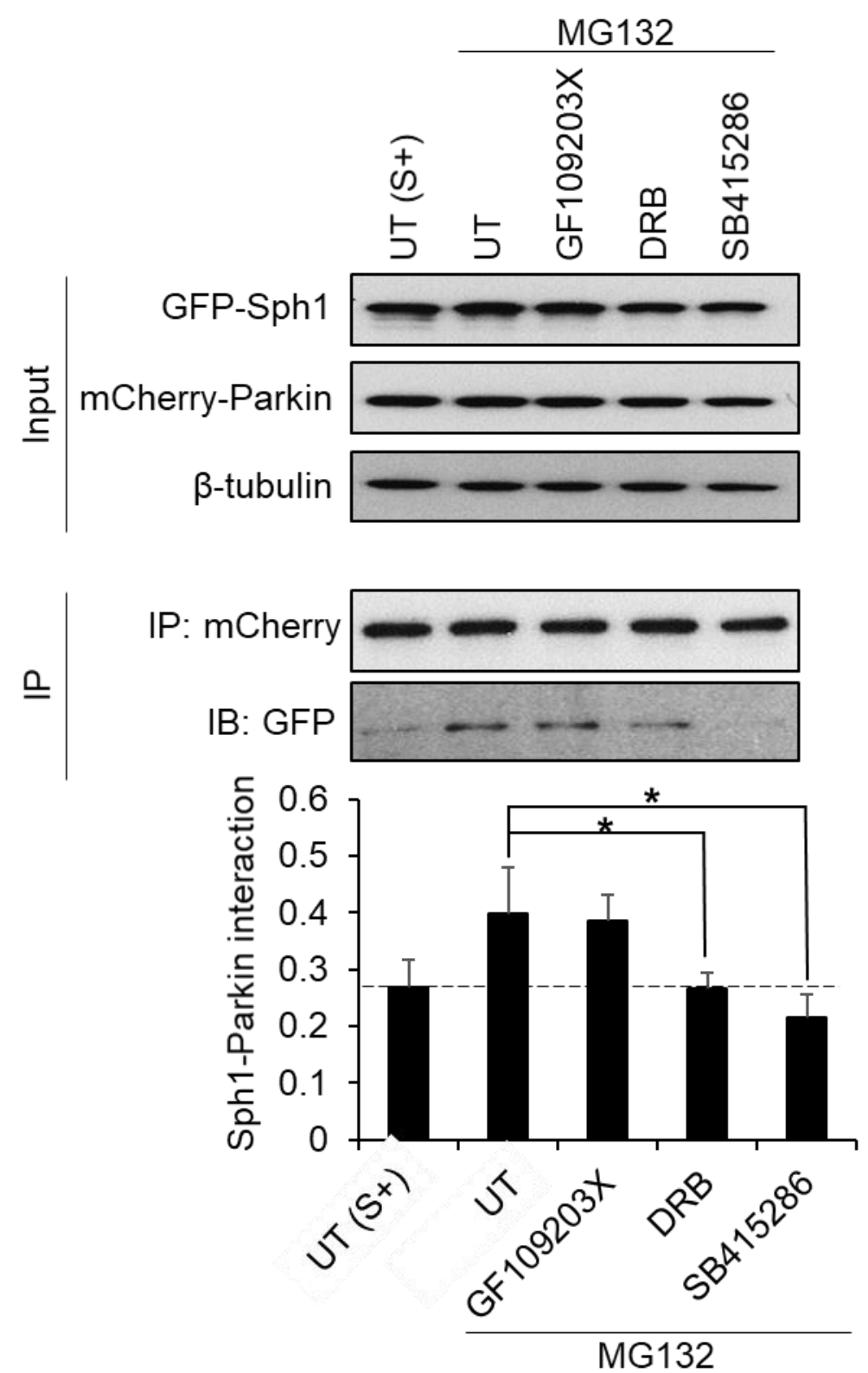
Figure 3.16 Inhibitions of CKII and GSK3 $\beta$ perturb the interaction between Parkin and Sph1 under proteasomal stress. GFP-Sph1 was transiently overexpressed in mCherry-Parkin stable cells for $24 \mathrm{~h}$. The media was then replaced with serum-rich $\mathrm{S}_{+}$ media in the absence and presence of $2 \mu \mathrm{M}$ MG132 for $16 \mathrm{~h}$. After this, the cells were treated with $1.0 \mu \mathrm{M}$ GF109203X, 50 $\mu \mathrm{M}$ DRB or $50 \mu \mathrm{M}$ SB415286 for another $12 \mathrm{~h}$. The cells were lysed with 1\% NP-40 lysis buffer, and the lysates were subjected to immunoprecipitation (IP) with anti-mCherry antibody, followed by immunoblotting (IB) with anti-GFP antibody. The upper panel (input) shows the total expression of mCherry-Parkin and GFP-Sph1 in the lysate. The lower panel (IP) shows the levels of mCherry-Parkin immunoprecipitated, and the levels of the GFP-Sph1 that co-immunoprecipitated with it. The bottom shows the graph for the quantification of the levels of GFP-Sph1 that coimmunoprecipitated with mCherry-Parkin under the different treatments. Values were expressed as mean + S.E.M ( $n=2-3)$. Differences against UT control under MG132 treatment are significant for ${ }^{*} \mathrm{p}<0.05$. 


\subsection{CKII AND GSK3 $\beta$ REGUALTES ACTIVATION OF PARKIN BY PHOSPHORYLATION}

Recently, it has been shown that phosphorylation events are important for activation of Parkin E3 ligase activity. Particularly, phosphorylation of Parkin at S65 and the addition of phosphorylated ubiquitin at $\mathbf{S} 65$ are important molecular events to fully activate Parkin to mediate mitophagy [395, 396].

With the earlier observations that PKCa and GSK3 $\beta$ interact with Parkin under proteasomal stress (Fig. $3.15 \mathrm{a}$ and d), we queried if these kinases modulate the activity of Parkin via phosphorylation to regulate aggrephagy. Particularly for GSK3 $\beta$, we hypothesized that the kinase is a novel regulator of Parkin phosphorylation to potentiate the addition of K63U linkages on Sph1 (Fig. 3.14b) to modulate inducible aggrephagy (Fig. 3.11d and 3.12d).

To explore whether PKC and GSK3 $\beta$ phosphorylate Parkin under proteasomal stress, we first examined the effects of these kinase inhibitions on the levels of phosphorylated Parkin $^{565}$ (P-Parkin ${ }^{565}$ ) and cytosolic phosphorylated ubiquitin ${ }^{\mathrm{S65}}$ (P-Ub ${ }^{\mathrm{S65}}$ ). Briefly, mCherry-Parkin stable cells were transiently transfected with GFP-Sph1 in the presence of proteasomal inhibitor MG132, and examined for the changes in levels of P-Parkin ${ }^{\$ 65}$ and $\mathrm{P}-\mathrm{Ub}^{565}$ under kinase inhibitions. Here, we not only focus on the effects of PKC and GSK3 $\beta$ inhibitions, but also examined whether CKII might regulate Parkin's activation.

Based on the results, CKII and GSK3 $\beta$ inhibitions significantly reduced the levels of $P$ Parkin ${ }^{565}$ by approximately 0.2 - and 0.4 -folds respectively when compared to the untreated (UT) cells, while minimal effect was observed for PKC inhibitor (Fig. 3.17a). On the other hand, inhibition of GSK3 $\beta$ markedly reduced the cellular levels of $\mathrm{P}-\mathrm{Ub}^{\mathrm{S65}}$ by approximately 0.4 -fold while PKC and CKII inhibitions did not significantly alter the levels (Fig. 3.17b). Taken together, these results suggest a preliminary mechanistic model where GSK3 $\beta$ may potentiate Parkin activation under proteasomal stress, by regulating 
the phosphorylation of Parkin at $\mathrm{S} 65$ and potentially the levels of $\mathrm{P}-\mathrm{Ub}^{\mathrm{S} 65}$ on Parkin. However, for the latter, it is important to examine the levels of $\mathrm{P}-\mathrm{Ub}^{\mathrm{S} 65}$ modifications on Parkin via the immunoprecipitation assay described in section 3.7 to confirm that GSK3 $\beta$ does indeed modulate the $\mathrm{P}-\mathrm{Ub}^{\mathrm{S65}}$ levels on Parkin. On the other hand, although interaction between CKII $\beta$ and Parkin was not observed (Fig, 3.15c), CKII inhibition perturbs phosphorylation of Parkin at S65. We postulated that the inhibition of the catalytic subunit CKIla (rather than CKII $\beta$ ) may account for the reduced Parkin phosphorylation under CKII inhibition. 

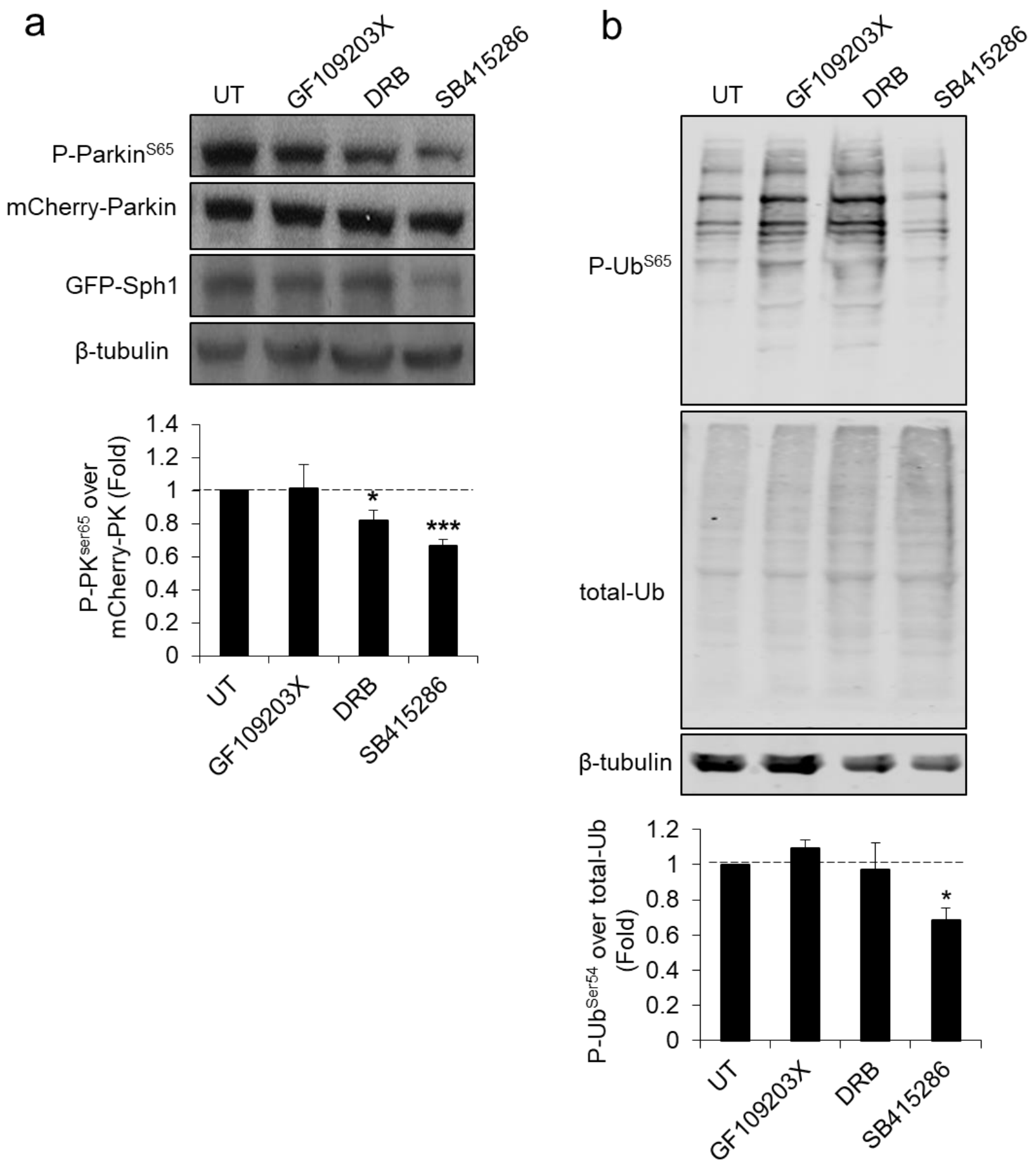
Figure 3.17 Inhibition of CKII reduces the levels of P-Parkin ${ }^{565}$, while inhibition of GSK3 $\beta$ perturbs P-Parkin ${ }^{565}$ and cellular $\mathrm{P} \mathrm{Ub}^{\mathbf{5 6 5}}$ levels. mCherry-Parkin stable cells were transiently transfected with GFP-Sph1 for $24 \mathrm{~h}$. The cells were then treated with $2 \mu \mathrm{M}$ MG132 proteasomal inhibitor for 16h, followed by treatment with 1.0 $\mu$ M GF109203X,

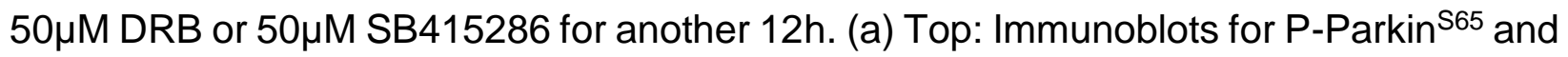
mCherry-Parkin levels under the different treatment conditions. Bottom: Graph showing the quantification of P-Parkin ${ }^{\mathrm{665}}$ levels. (b) Top: Immunoblots for P-Ub ${ }^{\mathrm{S65}}$ and total $\mathrm{Ub}$ levels under the different treatment conditions. Bottom: Graph showing the quantification of P-Ub ${ }^{S 65}$ levels. Fold values are expressed against UT control. Differences against UT are significant at ${ }^{*} p<0.05$ and ${ }^{* * *} p<0.0005$. 


\section{CHAPTER 4}

\section{POMEGRANATE ACTIVATES \\ TFEB TO PROMOTE \\ AUTOPHAGY-LYSOSOMAL \\ FITNESS AND MITOPHAGY FOR \\ MITOCHONDRIAL STRESS}




\subsection{BACKGROUND}

Polyphenols are natural compounds found in fruits and vegetables [397]. Diets enriched with these phenolics have been widely associated with human health benefits, including antioxidant, anti-inflammation and anti-cancer properties [16]. In both in-vivo and in-vitro systems, polyphenols have been shown to alleviate toxic amyloid load [346], and improve mitochondrial function by regulating mitochondrial metabolism and biogenesis [398, 399]. Thus, polyphenols display protective effects against proteotoxicity and mitochondrial dysfunction, the two common causative factors of aging and neurodegeneration [29, 30, 275, 400, 401].

Pomegranate is an example of polyphenol-enriched functional food. Multifaceted health benefits of pomegranate have been observed in various cell and animal models, as well as in human clinical trials [372, 373, 402-405]. Recent studies further demonstrated that pomegranate extract (PE) supplementation extended lifespan in $C$. elegans and drosophila [367, 406, 407]. Notably, many benefits of PE appear to overlap with autophagy regulation. Despite so, the molecular mechanism linking PE to autophagy remains inadequately understood.

Hence, this section focuses on investigating the effects of PE and its associated polyphenols on the general autophagy pathway. Additionally, efforts were directed at elucidating the underlying molecular mechanism for regulation of autophagy by PE. Furthermore, the ability of PE to mediate selective autophagic degradation of protein aggregates (aggrephagy) and damaged mitochondria (mitophagy) were also examined. With this, we hope to address the potential of modulating autophagy via dietary supplementation with PE, as a physiological therapeutic strategy to mitigate human diseases associated with proteotoxicity and mitochondrial dysfunction. 


\subsection{PE UPREGULATES AUTOPHAGY-LYSOSOMAL FITNESS}

Clearance of unwanted intracellular materials by autophagy represents an important pathway for cellular homeostasis in all cell types [408]. Amongst these, neuronal autophagy is especially important as a quality control pathway to maintain neuronal homeostasis, as post-mitotic neurons do not allow for removal of cytoplasmic toxic components via cell division [263, 409]. Here, we were interested to investigate the efficacy of a commercially available PE supplement in promoting cellular homeostasis in both neuronal and non-neuronal cells, by influencing the levels of autophagylysosomal components and autophagic activity.

\subsubsection{PE upregulates the levels of autophagosomes in multiple cell types}

Pomegranate and its polyphenolic components have been reported to induce autophagy in various cell types [410-412]. To verify the effect of PE on autophagy, we evaluated its effects on the autophagosomal compartment in multiple cell types, by examining the levels of LC3-II via immunoblotting (Fig. 4.1a). This analysis was performed in neuronal SY5Y cells and non-neuronal human embryonic kidney (HEK293) cells, human retina epithelial (RPE1) cells and Michigan Cancer Foundation-7 (MCF-7) mammary epithelium cancer cells. Cells were treated with PE dosages at 50,150 and $300 \mu \mathrm{g} / \mathrm{ml}$ for $24 \mathrm{~h}$. DMSO was used as the solvent vehicle control. LC3-II immunoblotting showed that various concentrations of PE markedly increased LC3-II levels in all cell types, except HEK293 cells, in comparison to their respective DMSO controls (Fig. 4.1a). Particularly, densitometry analyses revealed that PE significantly increased LC3-II levels in SY5Y and RPE1 cells under all PE treatments.

In HEK293 cells, while 50 to $300 \mu \mathrm{g} / \mathrm{ml}$ PE did not impact the LC3-II levels, increasing the PE dosages to 500 and $1000 \mu \mathrm{g} / \mathrm{ml}$ led to a significant 1 to 1.5 -folds increase in LC3-II levels compared to DMSO control (Fig. 4.1b). Hence, PE unanimously enhances the levels of autophagosomes across the different cell types analyzed, albeit at different concentrations. For the remaining study, the effects of PE on autophagy were mainly examined in SY5Y cells, as neuronal cells are particularly vulnerable to 
insults that perturb cellular homeostasis, an occurrence closely tied with dysfunctional autophagy activity [32].

Next, the time course effect of PE on the autophagosomal compartment was analyzed in SY5Y cells by treating the cells with $150 \mu \mathrm{g} / \mathrm{ml} \mathrm{PE}$ for 6, 12, 24 and 48h (Fig. 4.1c). LC3-II immunoblotting showed that PE increased the levels of LC3-II by approximately 1 -fold as early as $6 \mathrm{~h}$, with the effect sustained throughout the remaining period of investigation across 12, 24 and 48h (Fig. 4.1c). A similar increase in autophagosomes was detected in PE-treated SY5Y cells via immunofluorescence (IF) quantification of LC3 puncta (Fig. 4.1d). The IF results showed a significant 4-folds increase in the number of LC3 puncta in SY5Y cells upon 150 and $300 \mu \mathrm{g} / \mathrm{ml} \mathrm{PE}$ treatment for $24 \mathrm{~h}$ when compared to DMSO control. Taken together, these results indicate that PE upregulates the levels of autophagosomes in neuronal cells, and this response occurs as early as $6 \mathrm{~h}$ upon PE treatment. 

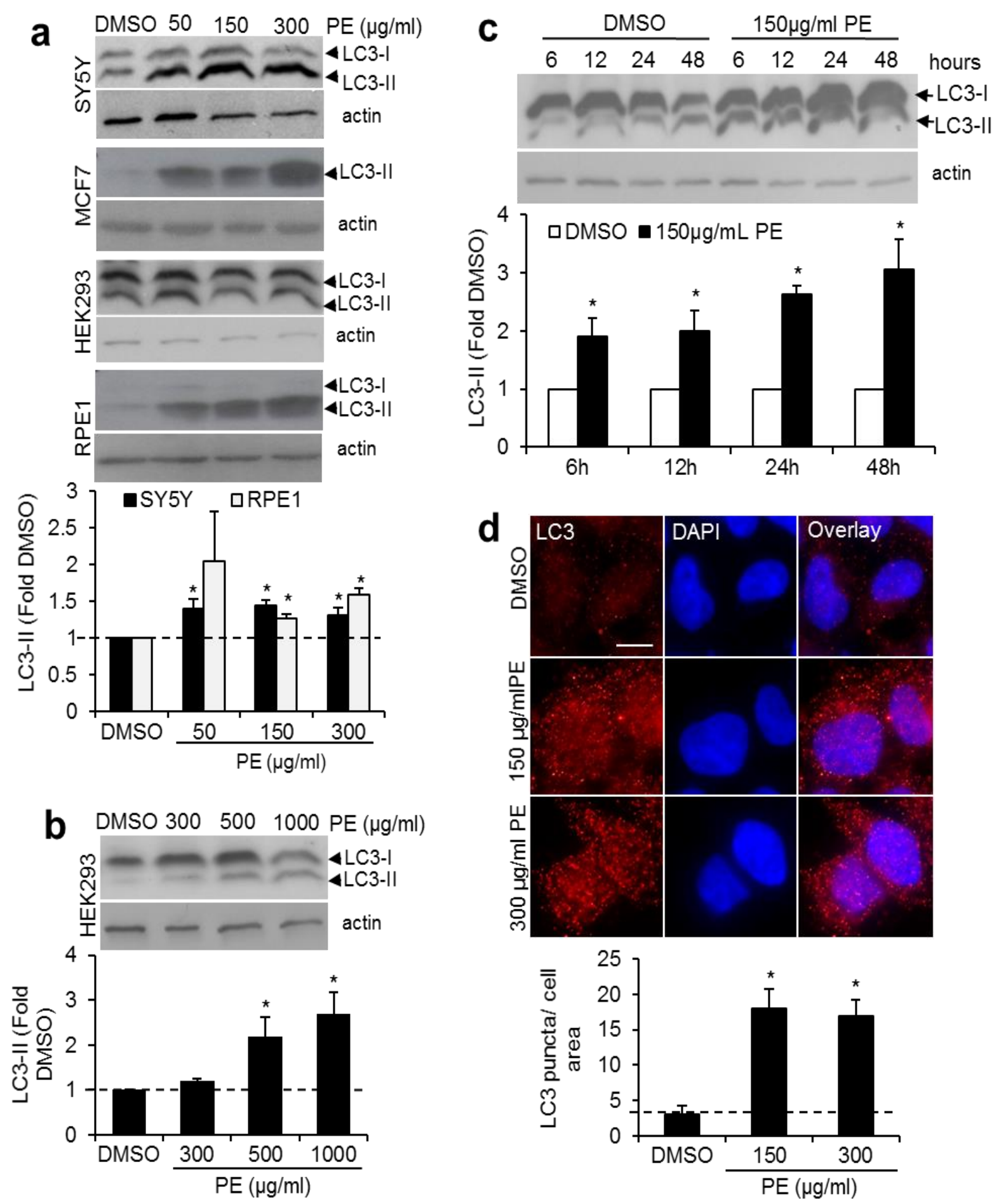
Figure 4.1 PE upregulates the autophagosomal compartment in multiple cell types. (a) Top: Immunoblots of LC3 in various cell lines treated with the vehicle control DMSO and increasing concentrations of PE at 50,150 and $300 \mu \mathrm{g} / \mathrm{ml}$ for $48 \mathrm{~h}$. Bottom: Quantification of LC3-II levels in neuronal SY5Y and non-neuronal RPE1 cells. (b) Top: Immunoblots of LC3 in HEK293 cells treated with DMSO, 300, 500 and $1000 \mu \mathrm{g} / \mathrm{ml} \mathrm{PE}$ for 48h. Bottom: Quantification of LC3-Il levels in HEK293 cells. (c) Top: Immunoblots of LC3-II in SY5Y cells treated with DMSO and 150 $\mu \mathrm{g} / \mathrm{ml} \mathrm{PE} \mathrm{for} \mathrm{6,} \mathrm{12,}$ 24 and 48h. Bottom: Quantification of LC3-II levels in SY5Y cells. (d) Immunofluorescence images (top) and quantification (bottom) of endogenous LC3 puncta in SY5Y cells treated with DMSO, 150 or $300 \mu \mathrm{g} / \mathrm{ml} \mathrm{PE}$ for $24 \mathrm{~h}$. At least 30 cells from random fields were analyzed for each condition. Nuclei were stained with DAPI. All values are mean + S.E.M $(n=3-4)$. Differences against DMSO control are significant for * $p<0.05$. Scale bars, $10 \mu \mathrm{m}$. 


\subsubsection{PE upregulates lysosomal compartment}

In addition to the increase in autophagosomal compartment, upregulation of the lysosomal compartment is another occurrence associated with autophagy induction. Hence, the effects of PE on the lysosomal compartment were analyzed by examining the LAMP1 levels in SY5Y cells treated with various concentrations of PE (Fig. 4.2). LAMP1 immunoblot analysis showed a significant 0.3 to 0.5 -fold increase in LAMP1 levels upon 24h PE treatments at 50, 150 and 300 $\mathrm{gg} / \mathrm{ml}$ (Fig. 4.2a).

Taken together, the commercial source PE health supplement used in this study significantly promotes autophagy-lysosomal fitness in SY5Y cells. To further validate that the enhanced autophagic response is specific to PE and not due to unidentified compounds in the commercial source PE (not specified by the manufacturer); we analyzed the effects of a HPLC-standardized extract of pomegranate that retains the natural polyphenolic ratio of whole pomegranate fruit (POMELLA, Verdure Sciences, Noblesville, USA) [368, 369] on the lysosomal compartment in SY5Y cells. Similar to the commercial source PE, scientific grade POMELLA induced an overt 1 to 1.5-folds increase in LAMP1 levels at $300-500 \mu \mathrm{g} / \mathrm{ml}$ (Fig. 4.2b). This result confirmed that the enhanced autophagic response induced by the commercial source PE is associated with the pomegranate components of the PE health supplement. The commercial source PE thus, sufficiently recapitulates the effects of scientific grade PE and affirms its application for the rest of this study.

With this validation, we went on to investigate the time course effects of PE on the LAMP1 levels. LAMP1 levels in SY5Y cells significantly doubled upon $150 \mu \mathrm{g} / \mathrm{ml}$ PE treatment from 12h onwards (Fig. 4.2c). In comparison to the PE's effect on the autophagosomes, PE-induced upregulation of LAMP1 represents a later response. IF analysis of LAMP1 puncta in SY5Y cells treated with 150 and $300 \mu \mathrm{g} / \mathrm{ml}$ PE showed an approximate 2 to 3 times expansion in the average size of the LAMP1 puncta as compared to DMSO control (Fig. 4.2d). The effects of PE on lysosomes were strikingly different from those observed with autophagosomes, where we saw an enhancement in the average size of the lysosomes rather than an increase in puncta number (Fig. 4.2d). PE therefore, upregulates both the autophagosomal and lysosomal 
compartments in SY5Y cells, although through slightly different mechanisms. While PE increases the number of autophagosomes, PE enhances lysosomal fitness by increasing the size of the lysosomes. 
a

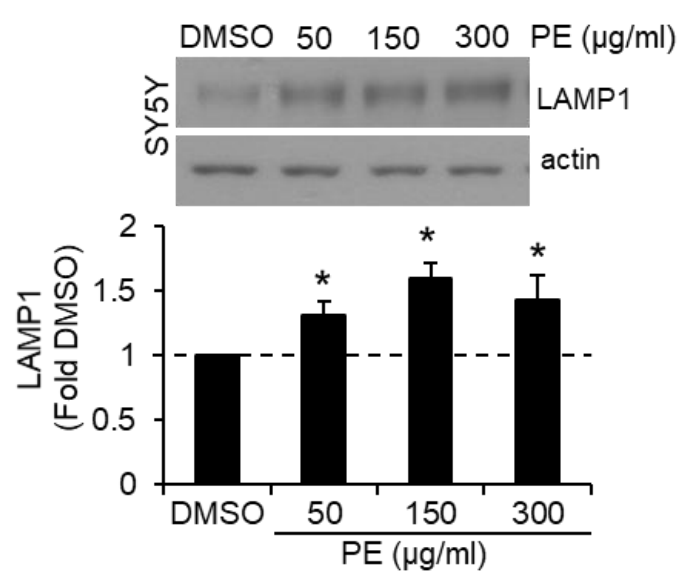

b

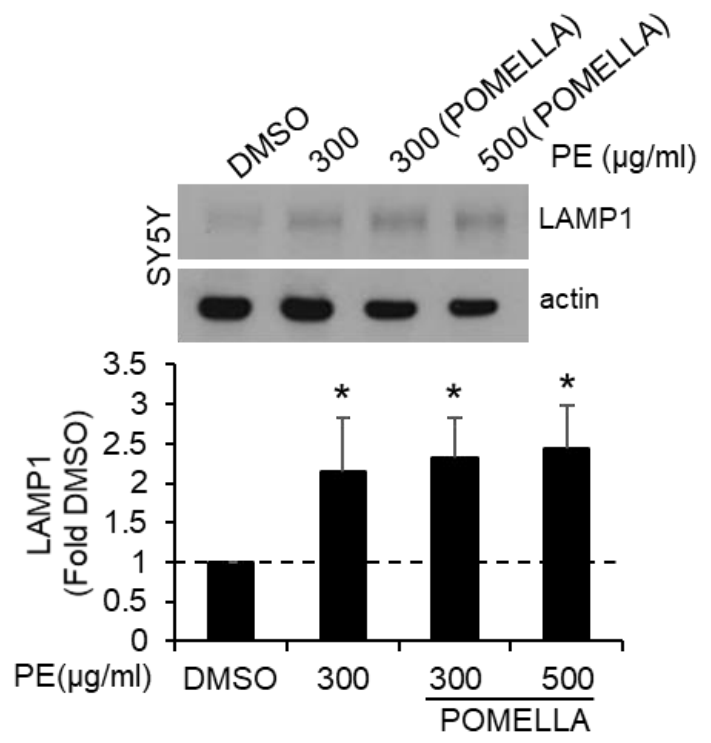

C
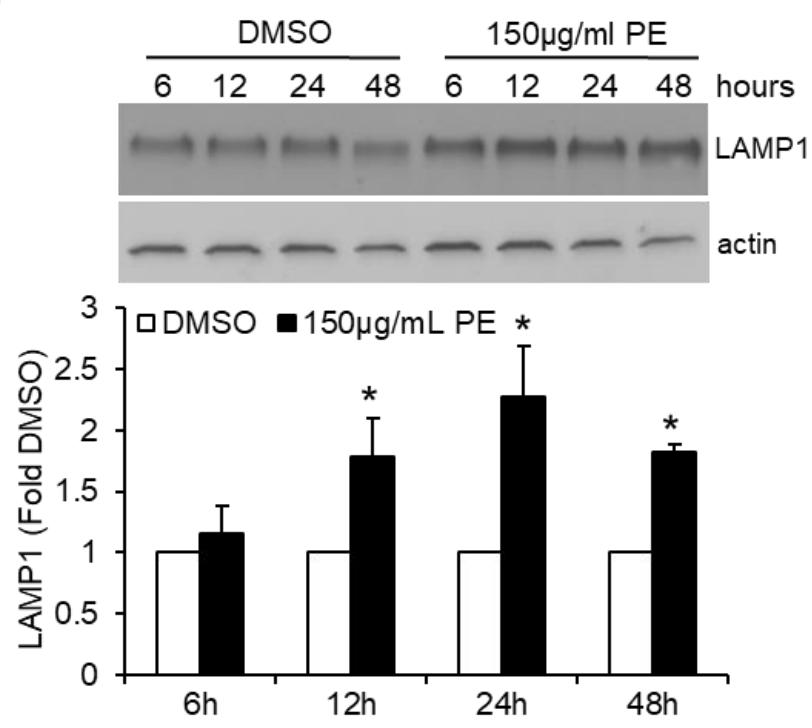

d
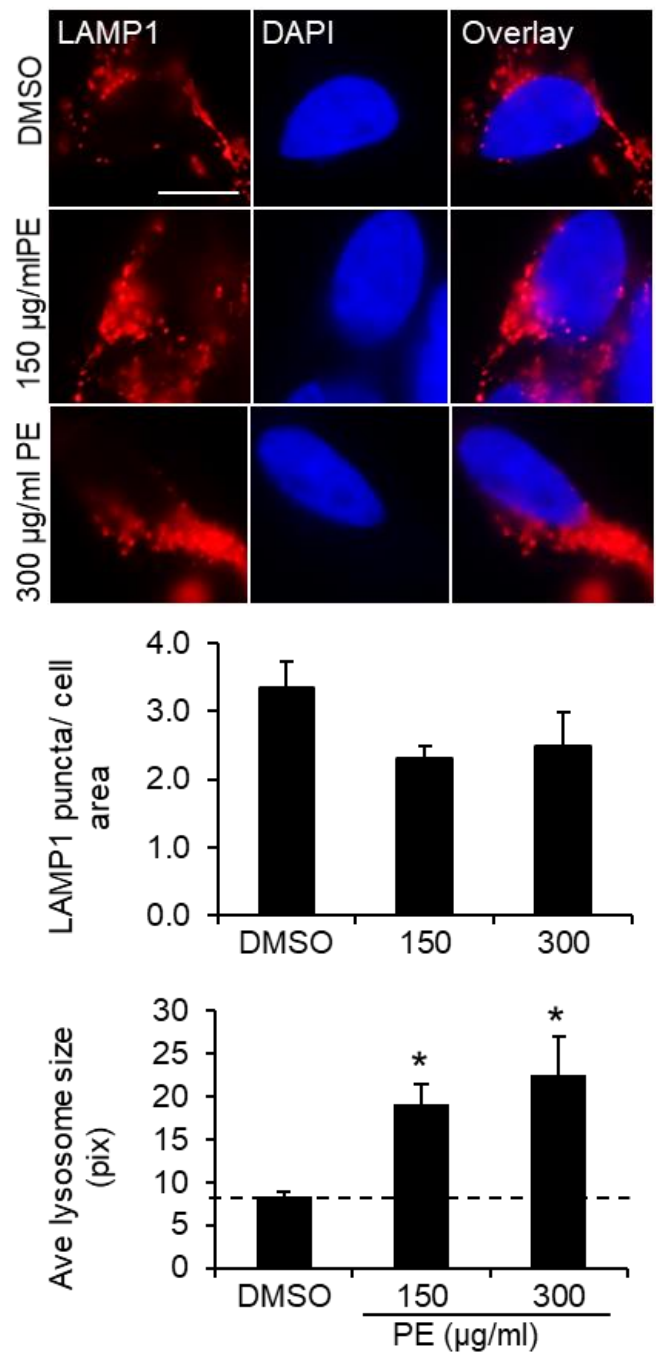
Figure 4.2 PE upregulates lysosomal compartment in SY5Y cells. (a) Top: Immunoblots of LAMP1 in SY5Y cells treated with DMSO and increasing concentrations of $P E$ at 50,150 and $300 \mu \mathrm{g} / \mathrm{ml}$ for $48 \mathrm{~h}$. Bottom: Quantification of LAMP1 levels in SY5Y cells. (b) Top: Immunoblots of LAMP1 in SY5Y cells treated with DMSO and $300 \mu \mathrm{g} / \mathrm{ml} \mathrm{PE}$, as well as increasing concentrations of POMELLA at 300 and $500 \mu \mathrm{g} / \mathrm{ml}$ for $24 \mathrm{~h}$. Bottom: Quantification of LAMP1 levels in SY5Y cells. (c) Top: Immunoblots of LAMP1 in SY5Y cells treated with DMSO and $150 \mu \mathrm{g} / \mathrm{ml} \mathrm{PE}$ for 6 , 12, 24 and 48h. Bottom: Quantification of LAMP1 levels in SY5Y cells. (d) Immunofluorescence images (top) and quantification (bottom) of endogenous LAMP1 puncta and area in SY5Y cells treated with DMSO, 150 or $300 \mu \mathrm{g} / \mathrm{ml} \mathrm{PE}$ for $24 \mathrm{~h}$. At least 30 cells from random fields were analyzed for each condition. Nuclei were stained with DAPI. All values are mean + S.E.M $(n=3-4)$. Differences against DMSO control are significant for ${ }^{*} p<0.05$. Scale bars, $10 \mu \mathrm{m}$. 


\subsubsection{Electron micrography analysis of PE-treated SY5Y cells}

Autophagy has been first characterized using electron microscopy (EM) [413]. Since then, EM has been commonly adopted to observe fine structures of the autophagic compartments [413-415]. Hence, we utilized EM as a complementary method to immunoblotting and IF to examine changes in the autophagy-lysosomal compartments upon PE treatment. Consistent with the higher levels of autophagosomes and lysosomes observed earlier (Fig. 4.1 and 4.2), EM analysis revealed a higher number of autophagic vacuoles (red arrows) and electron dense lysosomes (yellow arrows) in SY5Y cells treated with $150 \mu \mathrm{g} / \mathrm{ml}$ PE as compared to DMSO-treated control cells (Fig. 4.3). Together, our data strongly supports that PE upregulates both the autophagosomal and lysosomal compartments in SY5Y cells. 

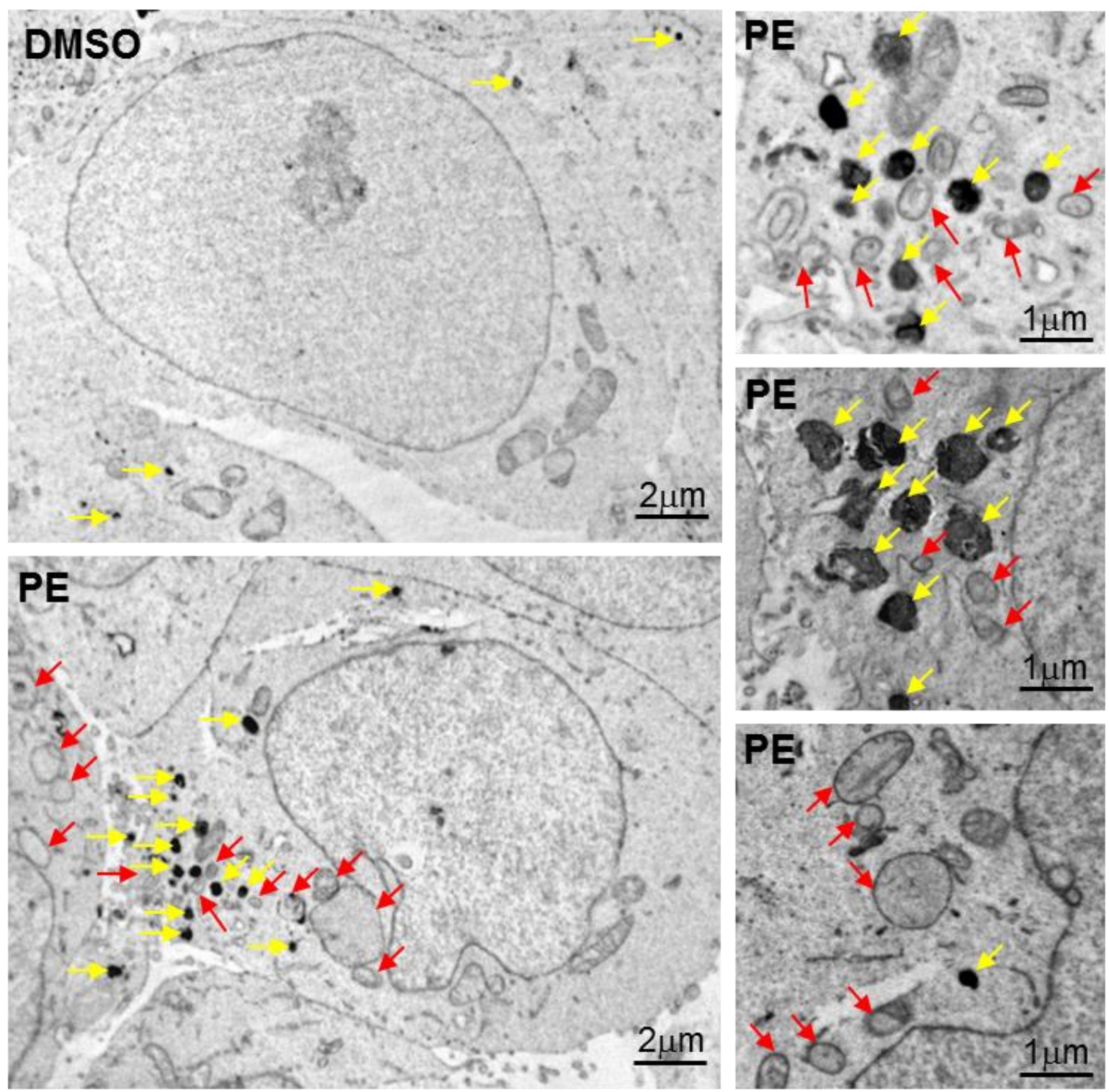

Figure 4.3 Electron microscopy analysis reveals high prevalence of autophagic vacuoles and lysosomes in PE-treated SY5Y cells. Electron micrographs of SY5Y cells treated with DMSO or $150 \mathrm{mg} / \mathrm{m}$ PE for $24 \mathrm{~h}$. Higher magnification fields show autophagic vacuoles (red arrows) and electron dense lysosomes (yellow arrows) in PE-treated SY5Y cells. Scale bars are expressed in $2 \mu \mathrm{m}$ or $1 \mu \mathrm{m}$ as stated. 


\subsubsection{PE enhances autophagy flux}

Higher autophagosomes and lysosomes can be associated with autophagy upregulation, or a consequence of autophagy dysfunction [416]. To verify that the higher autophagic structures observed with PE was due to higher autophagy activity and not a defect in turnover, the autophagy flux upon PE treatment was measured (Fig. 4.4). First, the tandem fluorescent mRFP-GFP-LC3 construct was used to monitor the autophagy flux via IF. The assay is based on the different $\mathrm{pH}$ stability of the GFP and RFP fluorescent proteins, where the former loses it fluorescence signal due to the lysosomal acidity. Hence, the construct can be used to label autophagosomes before and after fusion with the lysosomes [374]. LC3 puncta harboring the GFP signal will represent the autophagosomes. After fusion with the lysosomes, the GFP signal will be attenuated and the RFP-labelled LC3 puncta will indicate the autophagolysosomes (Fig. 4.4a) [374].

SY5Y cells were first transfected with the mRFP-GFP-LC3 construct followed by treatment with PE. The response of the tandem reporter to autophagic induction was verified by using the known autophagy stimulus, starvation (S-). Starvation significantly induced approximately 0.5 and 1 -fold increase in the number of autophagosomes (green) and autophagolysosomes (red) per cell area compared to basal serum-rich $(\mathrm{S}+)$ condition (Fig. 4.4b). Thus, the tandem reporter responds effectively to autophagic induction in SY5Y cells, and serves as a reliable indicator to measure autophagy flux in our cellular model. Similar to starvation response, distinct increase in the number of green and red LC3 puncta per cell area were also observed in SY5Y cells treated with 150 and $300 \mu \mathrm{g} / \mathrm{ml}$ PE for 24h (Fig. 4.4b).

Next, the turnover of LC3-II was analyzed via immunoblotting. LC3-II flux was measured by examining the magnitude of LC3-II accumulation under autophagic inhibition. Higher accumulation of LC3-II will indicate a higher rate of autophagosome turnover by the lysosomes (Fig. 4.4c). In our study, we used ammonium chloride and leupeptin (NL) to neutralize the lysosomal acidic $\mathrm{pH}$ and protease activities respectively to inhibit the lysosomes. 
In DMSO-treated control cells, LC3-II accumulated in the presence of $\mathrm{NL}(+\mathrm{NL})$ as compared to the untreated (-NL) condition. This accumulation represents the pool of autophagosomes that were turnover by the autophagy pathway basally, or the basal LC3-II flux in SY5Y cells. In comparison, 150 and $300 \mu \mathrm{g} / \mathrm{ml} \mathrm{PE} \mathrm{supplementation}$ induced 1 to 2-folds increase in the LC3-II flux when compared to DMSO-treated cells (Fig. 4.4d). Preliminarily, similar enhancement in LC3-II flux was also recapitulated with supplementation of $300 \mu \mathrm{g} / \mathrm{ml}$ POMELLA, the scientific grade PE, in SY5Y cells (Fig. 4.4e).

To summarize, PE-mediated increase in autophagosomal and lysosomal compartments is accompanied by higher LC3 flux, thus supporting that PE-induced autophagic response is associated with positive autophagy induction rather than dysfunction. 
a

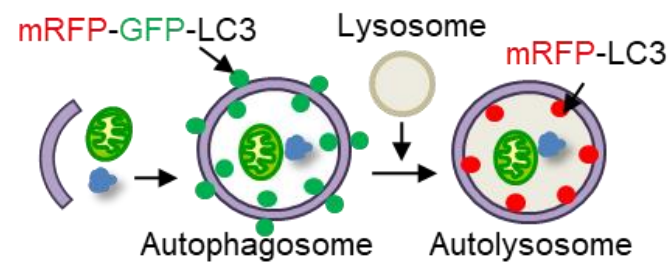

Autophagosome

\begin{tabular}{|c|c|c|}
\hline mRFP & GFP & LC3 \\
\hline $\begin{array}{l}\text { utophagosom } \\
\text { (Green) }\end{array}$ & & $\begin{array}{l}\text { Autolysosome } \\
\text { (Red) }\end{array}$ \\
\hline $\begin{array}{l}\text { Neutral pH } \\
\checkmark \text { Red } \\
\checkmark \text { Green }\end{array}$ & & $\begin{array}{l}\text { Acid pH } \\
\checkmark \text { Red } \\
\times \text { Green }\end{array}$ \\
\hline
\end{tabular}

b
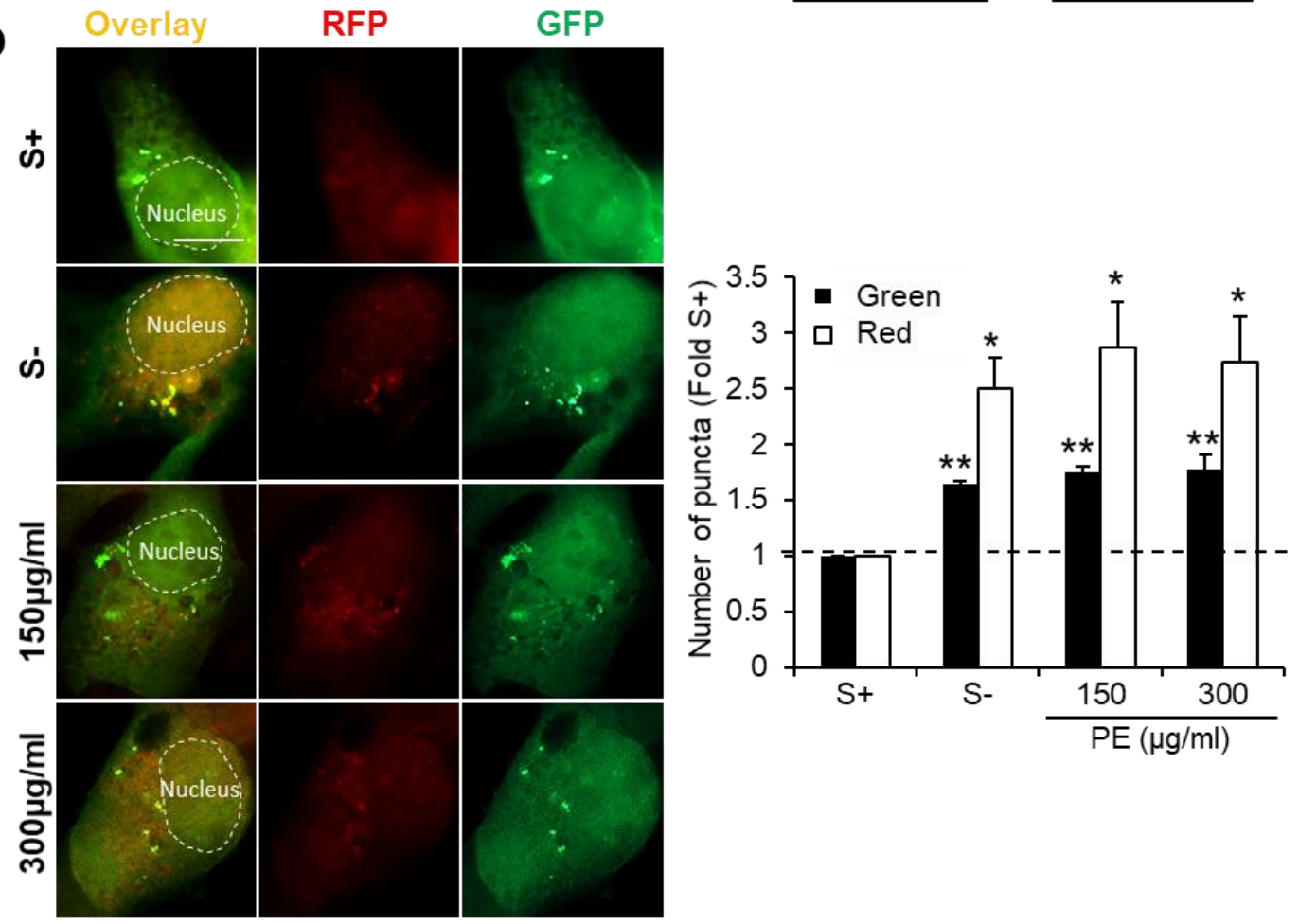

C

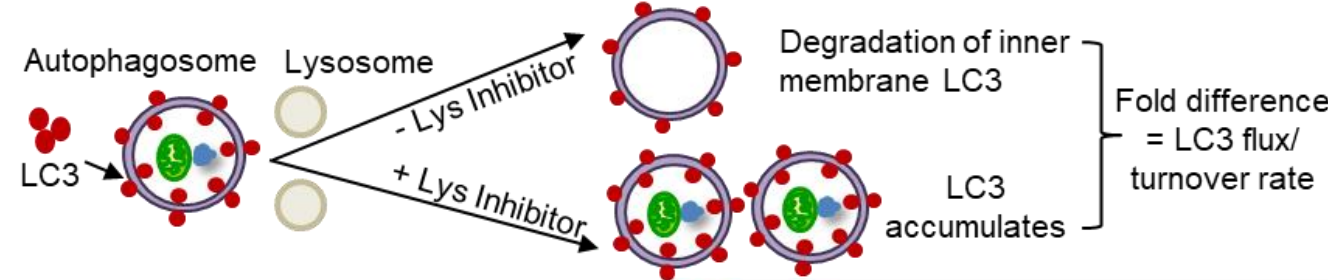

d
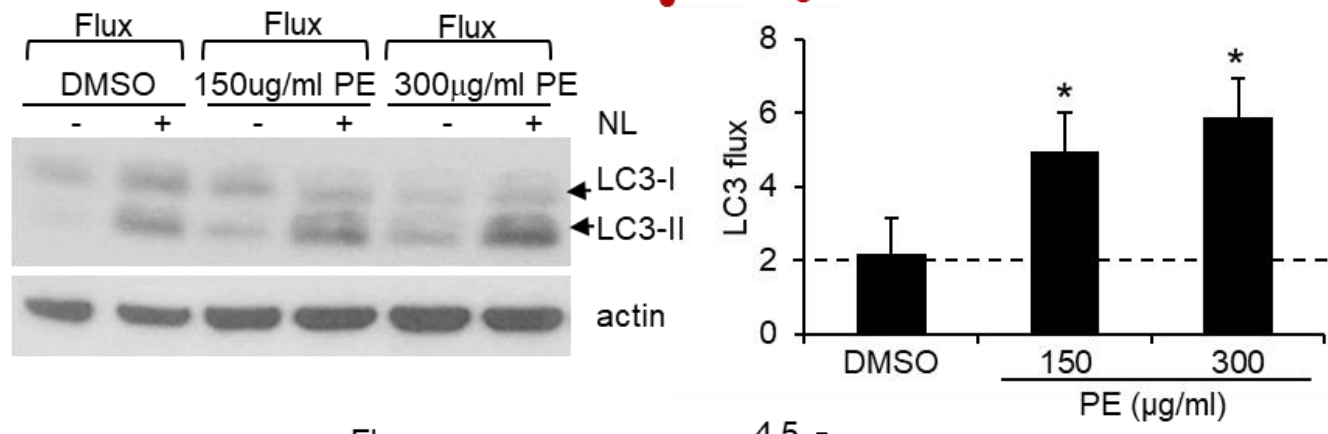

e
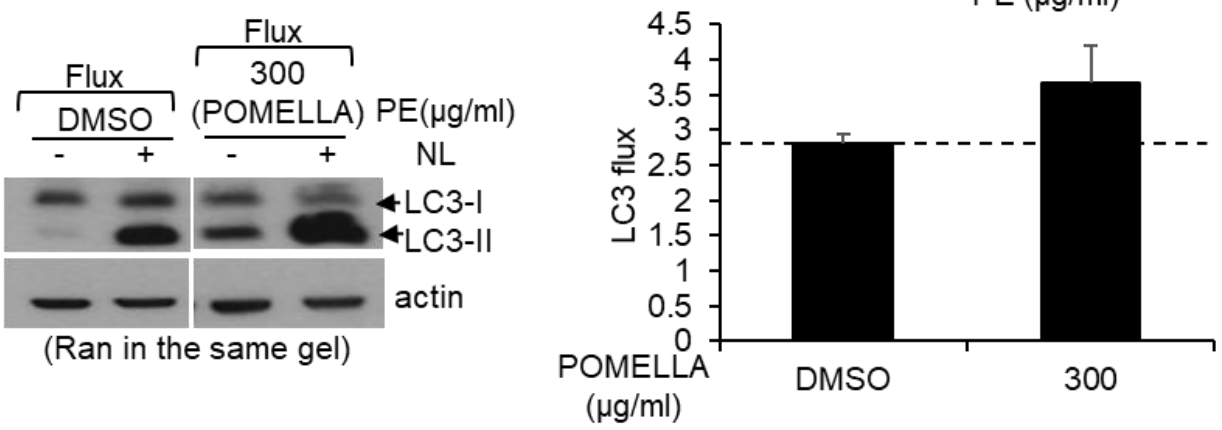
Figure 4.4 PE increases autophagic flux. (a) Schematic illustrating the principle of tandem mRFP-GFP-LC3 reporter assay for measuring autophagy flux. (b) Fluorescence images (left) and quantification (right) of green (autophagosome) and red (autolysosome) puncta in SY5Y cells transfected with tandem mRFP-GFP-LC3 reporter for $24 \mathrm{~h}$, followed by $24 \mathrm{~h}$ incubation under basal serum containing ( $\mathrm{S}+$ ), serum starvation (S-) or treatment with 150 or $300 \mu \mathrm{g} / \mathrm{ml}$ PE conditions. At least 30 transfected cells were analyzed for each condition. Nuclei were stained with DAPI. (c) Illustration for measurement of the autophagy flux. (d) Left: Immunoblot of LC3 in SY5Y cells treated with DMSO, 150 and $300 \mu \mathrm{g} / \mathrm{ml} \mathrm{PE}$ in the absence or presence of lysosomal

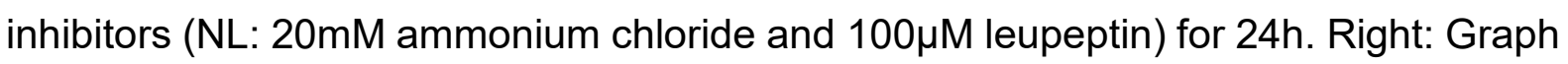
shows LC3-II flux calculated as fold increase in LC3-II levels upon lysosomal inhibition over LC3-II levels in the absence of NL. (e) Left: Immunoblot of LC3 in SY5Y cells treated with DMSO and $300 \mu \mathrm{g} / \mathrm{ml}$ POMELLA in the absence or presence of NL for 24h. Right: Graph shows LC3-II flux $(n=2)$. Values are mean + S.E.M $(n=3)$. Differences against $S+$ or DMSO controls are significant for ${ }^{*} p<0.05$ and ${ }^{* *} p<0.01$. Scale bar, $10 \mu \mathrm{m}$. 


\subsection{PE ACTIVATES TFEB SIGNALING}

Next, we examined the molecular mechanism underlying PE-stimulated autophagy. TFEB is a transcription factor that plays a pivotal role in autophagy regulation by promoting transcription of autophagy and lysosomal genes during nutrient deprivation $[90,103]$. As our earlier results demonstrate that PE upregulates both the autophagosomal and lysosomal compartments, we hypothesized that PE potentially upregulate autophagy via TFEB activation in SY5Y cells.

\subsubsection{PE promotes TFEB nuclear localization and activation}

One of the methods widely used to evaluate TFEB activation is to monitor its intracellular localization. Under nutrient-rich condition, TFEB is largely localized to the cytosol where it is kept inactive. Upon starvation-induced autophagy, TFEB rapidly translocate to the nucleus to activate transcription of autophagy and lysosomal genes. Hence, the nuclear localization of TFEB indicates its activation [91, 104, 119]. Using a stable GFP-TFEB expressing SY5Y cell line, we monitored the subcellular localization of TFEB upon PE treatment.

The responsiveness of GFP-TFEB stable cells to starvation was first validated with 24h S- treatment (Fig. 4.5). As reported [91], starvation markedly increased the percentage cells with TFEB nuclear localization from $8 \%$ (S+) to $28 \%$ (Fig. 4.5a). Next, treatment with 150 and $300 \mu \mathrm{g} / \mathrm{ml}$ PE also significantly enhanced TFEB nuclear shuffling as compared to DMSO control, a phenomenon associated with autophagy induction. Remarkably, both concentrations of PE consistently elicited a stronger TFEB activation response than S-, the known potent activator of TFEB (Fig. 4.5a).

Study in the HeLa cells demonstrated that starvation-induced TFEB activation is a rapid response [91]. To assess whether TFEB activation is a rapid response to $P E$, TFEB nuclear localization was monitored over a 6h short time-course PE treatment period (Fig. 4.5b). Time-dependent activation of TFEB was observed in both S- and PE paradigms. For S-, significant cytosol to nucleus TFEB shuffling was observed at 4h. In contrast, PE induced significant TFEB nuclear localization as early as upon $2 \mathrm{~h}$ 
treatment (Fig. 4.5b). Consistently, PE superseded S- in the magnitude of TFEB activation at all the time points studied.

Together, these observations demonstrate that PE activates TFEB nuclear translocation as early as $2 \mathrm{~h}$, which may contribute towards the upregulation of autophagy-lysosomal compartment observed at $6 \mathrm{~h}$ (Fig. 4.1c). Our observations also highlight that PE is a more potent activator of TFEB than starvation. 

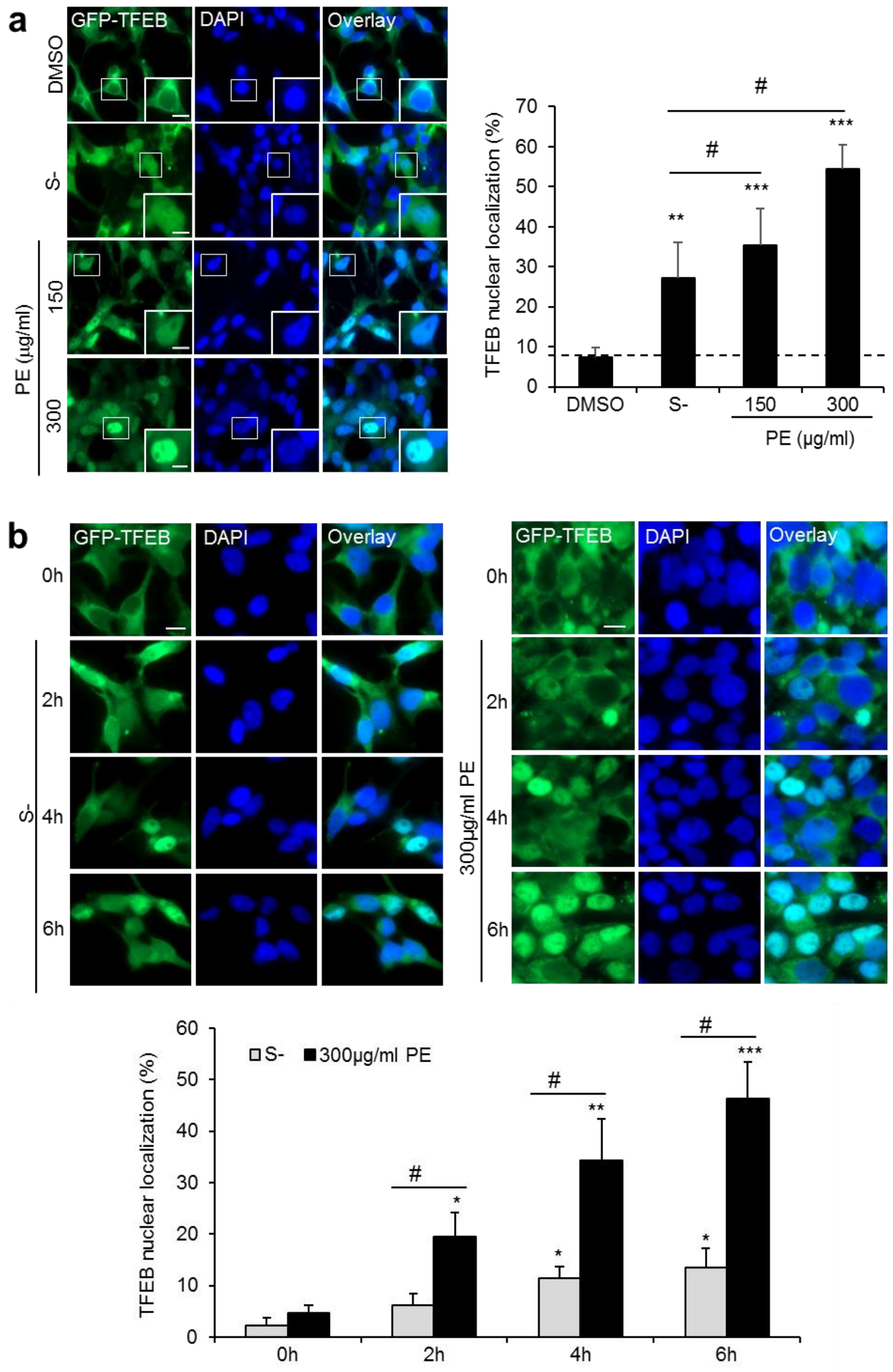
Figure 4.5 PE induces TFEB nuclear localization. (a) Top: Fluorescence images depicting localization of TFEB in GFP-TFEB SY5Y stable cells treated with DMSO, starvation (S-), 150 or $300 \mu \mathrm{g} / \mathrm{ml}$ PE for $24 \mathrm{~h}$. Nuclei were stained with DAPI. Bottom: Quantification of percentage TFEB nuclear localization in $\geq 100$ cells per condition. (b) Top: TFEB localization in GFP-TFEB SY5Y stable cells upon short-term starvation (S) or $300 \mu \mathrm{g} / \mathrm{ml}$ PE treatment for $0,2,4$, and 6h. Bottom: Quantification of percentage TFEB nuclear localization in at least 100 transfected cells per condition for short-term treatment. Differences against DMSO or $\mathrm{S}$ - are significant at ${ }^{*} p<0.05,{ }^{* *} p<0.01$, ${ }^{* * *}$ $p<0.0005$ and $\# p<0.05$ respectively. Scale bar, $10 \mu \mathrm{m}$. 


\subsubsection{PE activates TFEB via TFEB ${ }^{S 142}$ dephosphorylation}

Spatial localization of TFEB is regulated by phosphorylation events. Phosphorylation of TFEB at S142 and/or S211 promotes its cytosolic sequestration, while starvation reduces these inhibitory phosphorylations on TFEB to activate TFEB nuclear translocation [91, 117-119]. With the observation that PE induces TFEB nuclear shuffling, we assessed if PE-induced TFEB activation is regulated by phosphorylation.

Using the antibody against phosphorylated TFEB ${ }^{S 142}$ (P-TFEB ${ }^{S 142}$, the only antibody available thus far for phosphorylated form of TFEB, we examined if PE-induced TFEB activation is regulated by $\mathrm{S} 142$ phosphorylation. To ensure that changes in P-TFEB ${ }^{\mathrm{S} 142}$ levels is not due to changes in the total TFEB (T-TFEB) levels, T-TFEB levels were analyzed by probing for anti-GFP-TFEB. P-TFEB ${ }^{\mathrm{S} 142}$ was normalized against T-TFEB for quantification.

Consistent with the reported findings $[118,119], 6 \mathrm{~h}$ and $24 \mathrm{~h} \mathrm{~S}$ - treatment significantly reduced P-TFEB ${ }^{S 142}$ levels by 0.2 and 0.4 -fold respectively as compared to DMSO control (Fig. 4.6). This result coincided with the enhanced TFEB nuclear localization observed at the same time points in Fig. 4.5. A similar but more dramatic reduction in P-TFEB ${ }^{S 142}$ levels was detected for PE treatment at both time points as compared to S- (Fig. 4.6). Thus, PE mechanistically activates TFEB by reducing the inhibitory $S 142$ TFEB phosphorylation to facilitate TFEB nuclear translocation. 

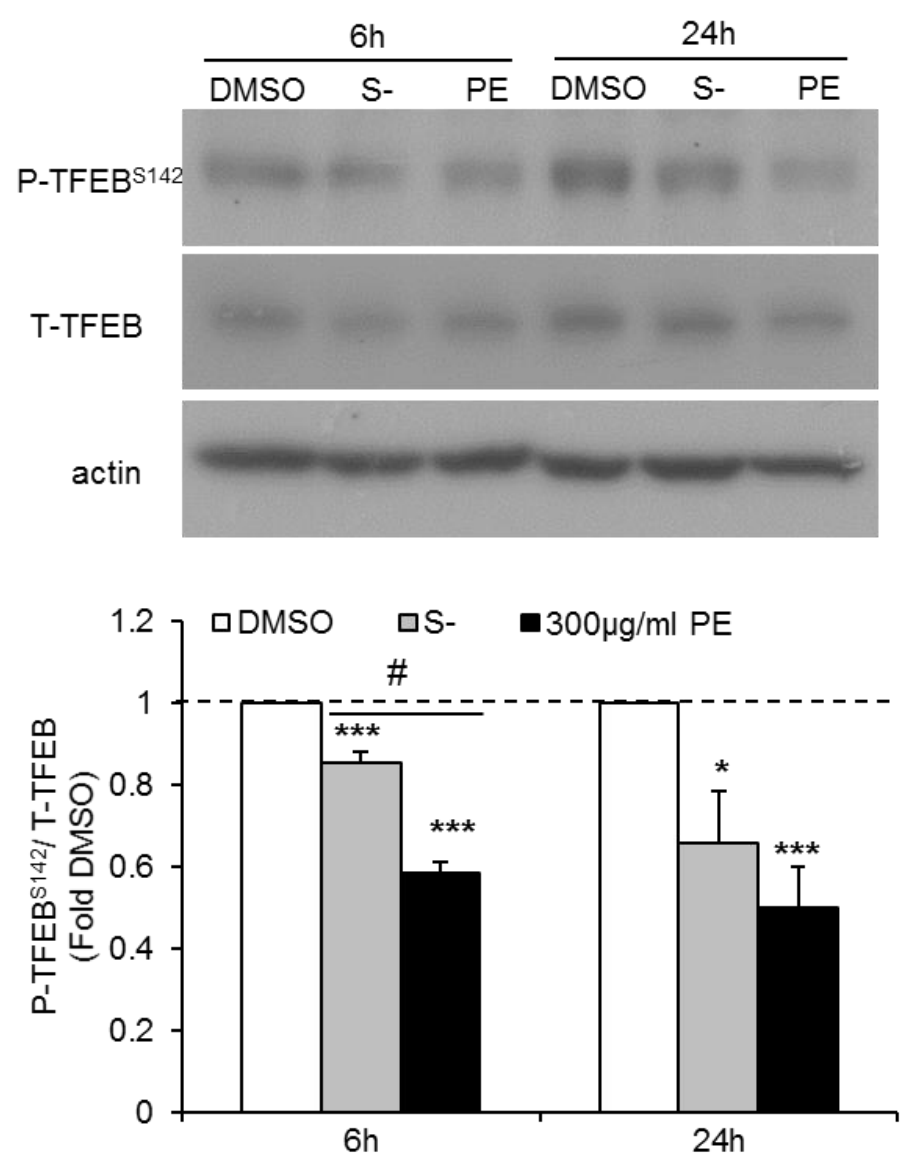

Figure 4.6 PE reduces TFEB $^{\mathrm{S} 142}$ phosphorylation. Top: Immunoblots of phosphorylated TFEB at S142 (P-TFEB ${ }^{\text {S142 }}$ ) and total TFEB (T-TFEB) in cells treated with DMSO, S- or $300 \mu \mathrm{g} / \mathrm{ml} \mathrm{PE}$ for $6 \mathrm{~h}$ and $24 \mathrm{~h}$. Bottom: Quantification of P-TFEB ${ }^{\mathrm{S} 142}$ levels. All values are mean + S.E.M $(n=3-12)$. Fold values are expressed against DMSO control. Differences against DMSO or $S$ - are significant at ${ }^{*} p<0.05$, ${ }^{* * *}$ $p<0.0005$ and $\# p<0.05$ respectively. 


\subsection{LYSOSOMAL-Ca ${ }^{2+}$ SIGNALING PATHWAY UNDERLIES PE-INDUCED TFEB ACTIVATION}

TFEB phosphorylation is regulated by kinases and phosphatase, including ERK2, mTORC1 and $\mathrm{Ca}^{2+}$-activated calcineurin. mTORC1 and ERK2-mediated phosphorylations at $\mathrm{S} 142$ and S211 sequester the TFEB in the cytosol via binding with cytoplasmic 14-3-3 protein [91, 117-119]. On the other hand, $\mathrm{Ca}^{2+}$-activated calcineurin dephosphorylates TFEB at S142 and S211 to induce nuclear shuttling during starvation [120]. With the observation that PE-induced TFEB nuclear localization was due to changes in TFEB phosphorylation status, we were interested to examine the regulatory mechanism that controls TFEB dephosphorylation and nucleus shuffling under PE treatment.

\subsubsection{PE-induced TFEB activation is not dependent on ERK2 signaling}

ERK1/2 is activated via phosphorylation at T202 and Y204 [417]. To determine whether PE modulates TFEB activation via ERK1/2 signaling, the levels of active phosphorylated ERK1/2 were analyzed upon 150 and $300 \mu \mathrm{g} / \mathrm{ml} \mathrm{PE}$ treatment (Fig. 4.7). Neither of the PE dosages influenced the levels of phosphorylated ERK1/2 compared to DMSO control cells. Thus, PE does not affect ERK1/2 signaling and is unlikely to regulate TFEB through this pathway. 

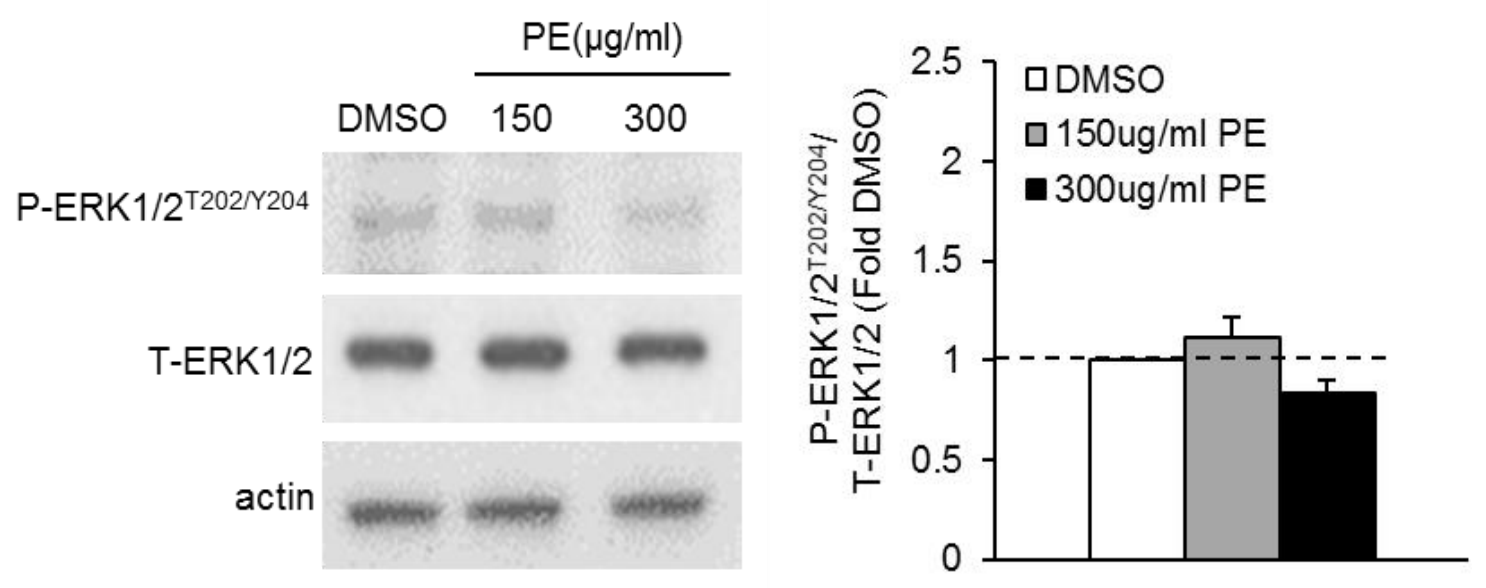

Figure 4.7 PE does not influence ERK1/2 activation and signaling. Top:

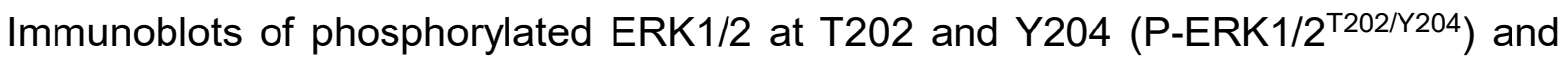
total ERK1/2 (T-ERK1/2) in SY5Y cells treated with DMSO, 150 or $300 \mu \mathrm{g} / \mathrm{ml}$ PE for

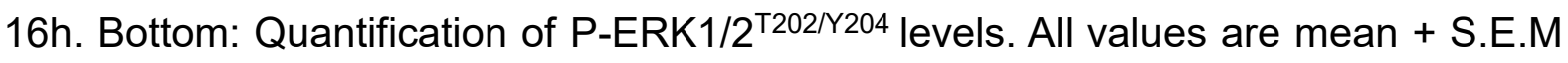
$(n=3)$. Fold values are expressed against DMSO control. 


\subsubsection{PE-induced TFEB activation is independent of AKT-mTORC1 signaling}

Under nutrient-rich condition, mTORC1 is activated by phosphorylation on mTOR at S2448 by AKT. The latter is in turn, regulated by phosphorylation at T308 and S473 to inhibit autophagy [418]. Active mTORC1 phosphorylates and inhibits TFEB nuclear localization, and shuts down autophagy activation [118]. We therefore examined whether PE influences mTORC1 via the AKT-mTORC1 signaling axis to regulate TFEB dephosphorylation and activation.

We first looked at the phosphorylation and activation of AKT, the upstream regulator of mTORC1 under 150 and $300 \mu \mathrm{g} / \mathrm{ml} \mathrm{PE}$ treatments. $300 \mu \mathrm{g} / \mathrm{m}$ PE significantly enhanced the levels of phosphorylated $A K T^{T 308}$ and $A K T^{S 473}$ by 1 and 0.5 -fold respectively as compared to DMSO control cells (Fig. 4.8a). In line with this activation, mTOR phosphorylation at S2448 was also enhanced by approximately 1 -fold with $300 \mu \mathrm{g} / \mathrm{m}$ PE (Fig. 4.8b). To confirm the activation of mTORC1 upon PE treatment, we checked for the activation of mTORC1 effector p70/S6K. The phosphorylated p70/S6K levels were also enhanced by 0.5 -fold with $300 \mu \mathrm{g} / \mathrm{m}$ PE as compared to DMSO control cells (Fig. 4.8c).

Together, these results demonstrate that PE activates AKT-mTORC1 signaling axis. However, it is surprising as $\mathrm{mTORC} 1$ is reported to negatively regulate TFEB nuclear localization and suppresses autophagy [119]. Alternatively, we hypothesized that PE regulates TFEB dephosphorylation and activation via another route of mechanism, which could bypass or outweigh the inhibitory effect of mTORC1 on TFEB. 
a

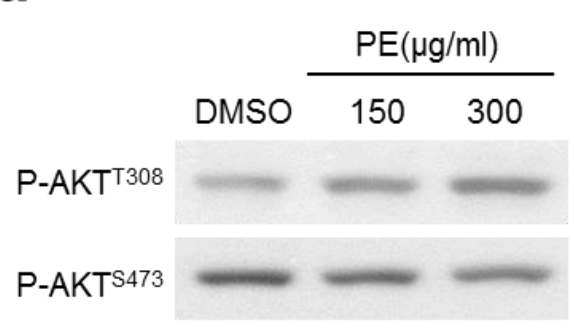

T-AKT

actin

b

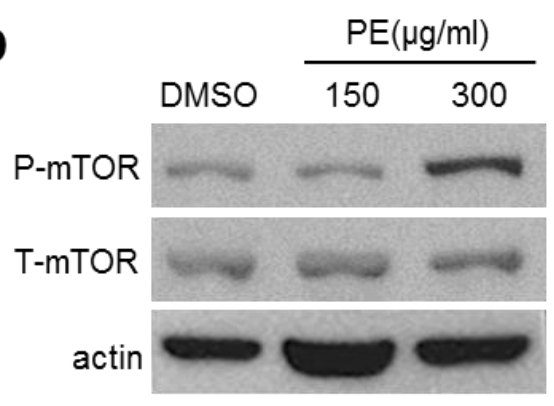

C

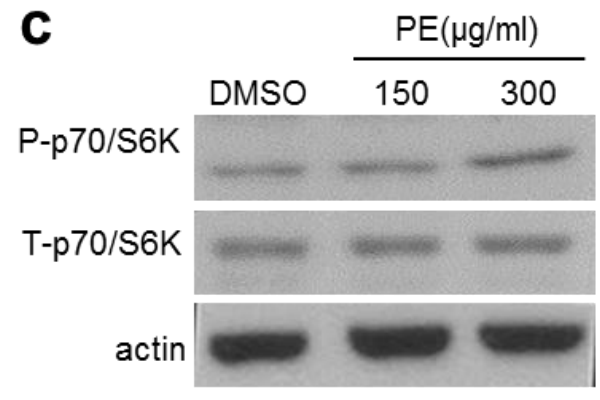

口DMSO

150ug/ml PE

- 300ug/ml PE
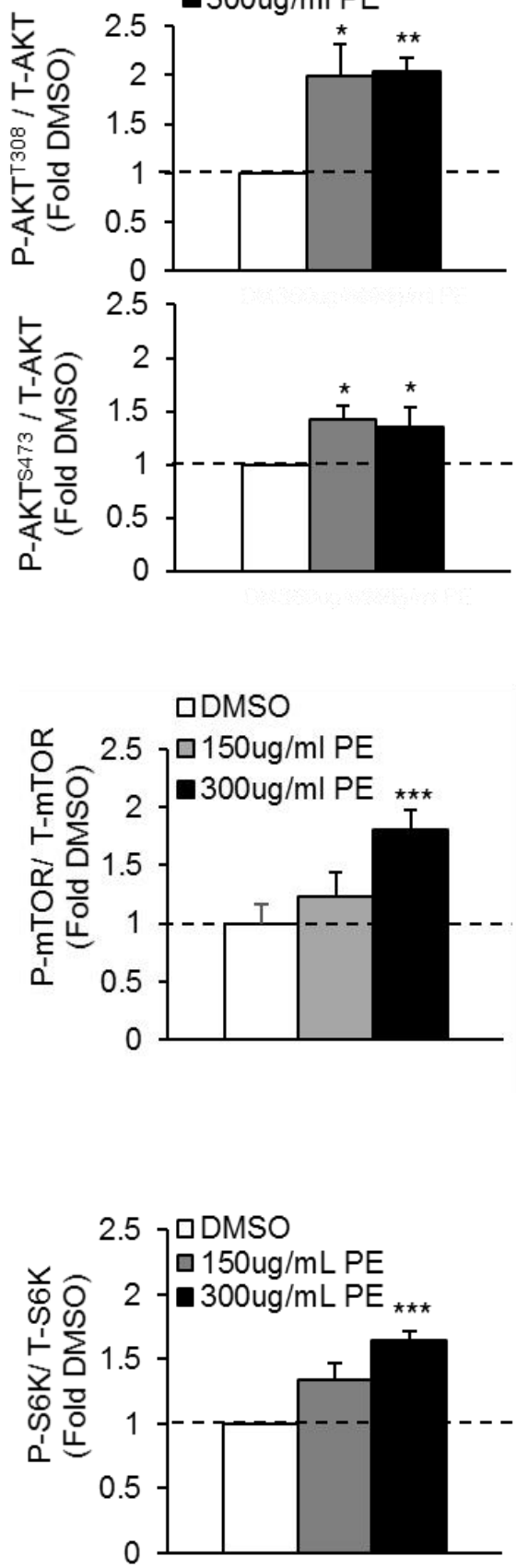
Figure 4.8 PE activates AKT and mTORC1 signaling pathways. (a-c) Left: Immunoblots of phosphorylated (P) and total (T) forms of (a) AKT and (b) mTOR and its downstream target (c) p70/S6K, in SY5Y cells treated with DMSO, 150 or $300 \mu \mathrm{g} / \mathrm{ml}$ PE for 16h. Right: Quantification of various phosphorylated proteins against their respective total proteins upon normalization with actin. Values are represented as mean + S.E.M $(n=3-7)$. Differences against DMSO are significant for ${ }^{*} p<0.05$, ** $p<0.01,{ }^{* * *} p<0.0005$. 


\subsubsection{PE activates Iysosomal- $\mathrm{Ca}^{2+}$ signaling pathway}

The lysosomal- $\mathrm{Ca}^{2+}$ signaling pathway facilitates TFEB nuclear localization independent of mTORC1 activity under starvation [120]. In this pathway, $\mathrm{Ca}^{2+}$ release from the lysosomal compartment activates the phosphatase calcineurin in the cytosol, which dephosphorylates and induces translocation of TFEB from the surface of lysosome to the nucleus [120]. To assess whether intracellular $\mathrm{Ca}^{2+}$ levels as well as calcineurin activity influence PE-induced TFEB nuclear localization, we modulated the cytoplasmic $\mathrm{Ca}^{2+}$ levels and inhibited the calcineurin activity with $\mathrm{Ca}^{2+}$ chelator BAPTA-AM and FK506 respectively, and examined their effects on PE-induced TFEB nuclear localization [120].

Prior to analyzing the effects inhibiting lysosomal-Ca ${ }^{2+}$ signaling on PE-induced TFEB activation, optimal concentrations of FK506 and BAPTA-AM that effectively blocked starvation-induced TFEB nuclear translocation were first determined. GFP-TFEB SY5Y stable cells were starved in the absence or presence of $10 \mu \mathrm{M}$ BAPTA-AM or $10 \mu \mathrm{M}$ FK506 for $16 \mathrm{~h}$, and the percentage of cells displaying nuclear TFEB localization under each condition were quantified. Treatment with both drugs effectively reduced starvation-induced TFEB nuclear translocation from approximately $12 \%$ to $<5 \%$ (Fig. 4.9a and b). Similarly, both inhibitors also abrogated PE-induced TFEB nuclear localization. While $60 \%$ nuclear localized TFEB cells were observed with $300 \mu \mathrm{g} / \mathrm{ml}$ PE for 16h, BAPTA-AM or FK506 addition significantly reduced the percentages to 55\% and $40 \%$ respectively (Fig. $4.9 \mathrm{a}$ and b). Thus, inhibition of the lysosomal-Ca ${ }^{2+}$ signaling pathway perturbs PE-induced TFEB activation.

$\mathrm{Ca}^{2+}$-activated calcineurin was shown to dephosphorylate TFEB at S142 to induce nuclear shuttling under starvation [120]. To further support that lysosomal-Ca ${ }^{2+}$ signaling underscores PE-induced TFEB nuclear localization, the effects of calcineurin inhibition on starvation- and PE-induced TFEB dephosphorylation were analyzed upon 5 and $10 \mu \mathrm{M}$ FK506 treatment for 16h (Fig. 4.9c). Both FK506 concentrations attenuated TFEB dephosphorylation induced by S- and PE. This data is consistent with the observation of reduced PE-induced TFEB nuclear translocation under calcineurin inhibition. 
Together, our findings strongly suggest that PE-induced TFEB nuclear translocation is dependent on intracellular $\mathrm{Ca}^{2+}$ levels and calcineurin activity. This further implies that PE potentiates TFEB nuclear translocation and activation via control of lysosomal $\mathrm{Ca}^{2+}$ signaling pathway to upregulate autophagy. 

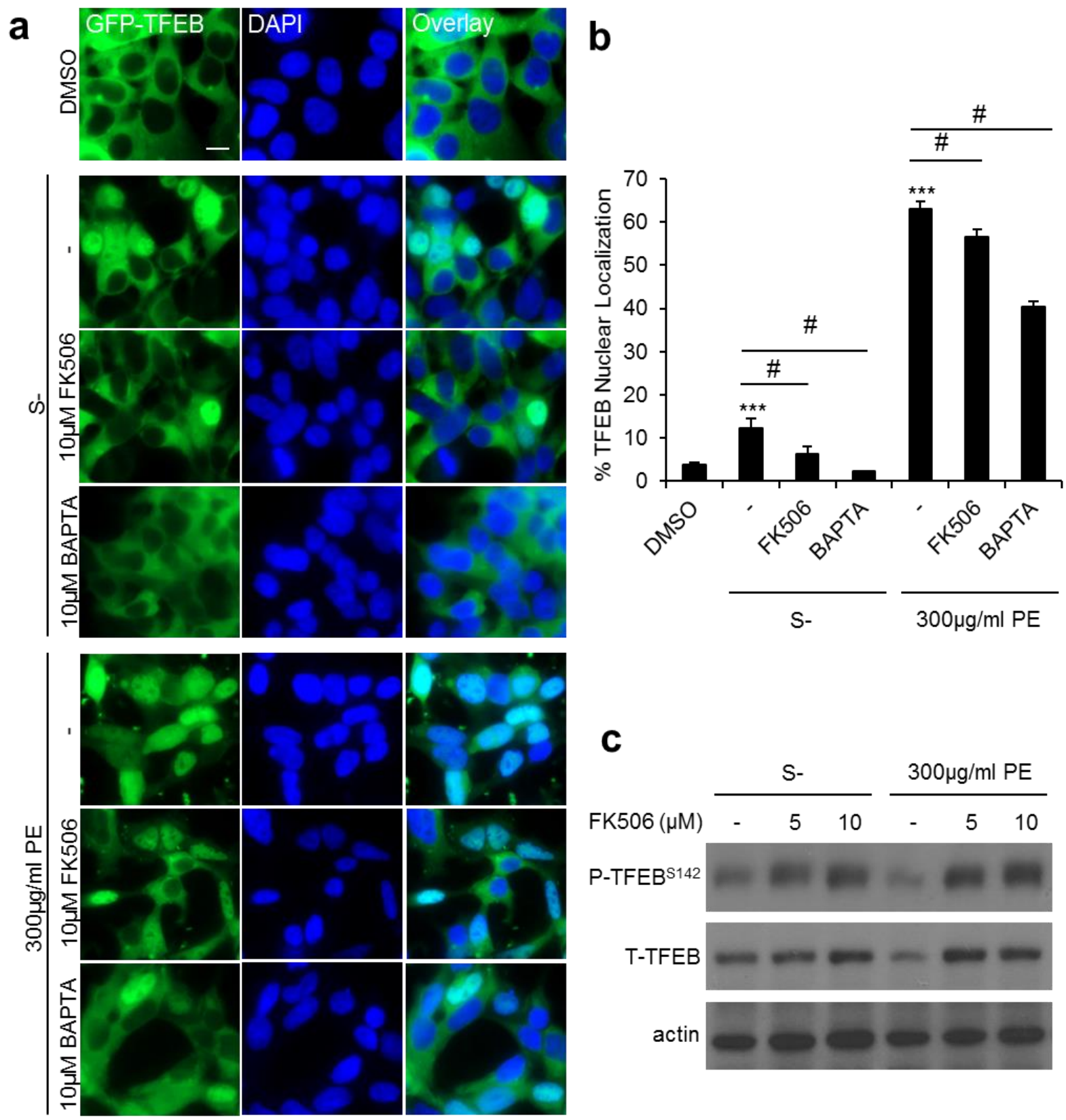
Figure 4.9 PE activates TFEB via $\mathrm{Ca}^{2+}$-calcineurin signaling. (a) Fluorescence images of TFEB localization in GFP-TFEB SY5Y stable cells treated with DMSO as well as S- or $300 \mu \mathrm{g} / \mathrm{m} \mathrm{PE}$ in the absence or presence of $10 \mu \mathrm{M}$ FK506 (calcineurin inhibitor) or $10 \mu \mathrm{M}$ BAPTA ( $\mathrm{Ca}^{2+}$ chelator) for $16 \mathrm{~h}$. Nuclei were stained with DAPI. (b) Quantification of percentage TFEB nuclear localization in at least 100 transfected cells per condition. (c) Immunoblots of P-TFEB ${ }^{\mathrm{S} 142}$ and T-TFEB in SY5Y cells treated with increasing concentrations of FK506. All values are mean + S.E.M ( $n=3-4)$. Differences between DMSO are significant at ${ }^{* * *} p<0.0005$. FK506 and BAPTA inhibitions of TFEB nuclear localization under $\mathrm{S}$ - and $\mathrm{PE}$ conditions are significant against untreated for \# $\mathrm{p}<0.05$. 


\subsection{PE PROTECTS AGAINST OXIDATIVE STRESS DAMAGE AND PROTEIN AGGREGATION INDEPENDENT OF AUTOPHAGY}

With the observation that PE activates TFEB via lysosomal- $\mathrm{Ca}^{2+}$ signaling to potentiate autophagy induction, we next investigated the functional significance of PEinduced autophagy in SY5Y cells. Proteotoxicity and mitochondrial dysfunction are widely associated with neurodegeneration and aging [29, 30, 400, 401]. PE and its phenolics have been shown to alleviate protein aggregation and improve mitochondrial function [366, 398, 399]. Hence, we were keen to examine whether PE promotes cellular homeostasis and protects against cellular dysfunction by facilitating selective autophagic degradation of protein aggregates and damaged mitochondria.

\subsubsection{PE reduces $\mathrm{H}_{2} \mathrm{O}_{2}$-induced protein oxidation and enhances cell viability independent of the autophagy pathway}

Oxidative stress is a major cause of protein damage [419]. To understand whether PE-induced autophagy alleviates proteotoxicity by promoting clearance of damaged proteins, SY5Y cells were treated with hydrogen peroxide $\left(\mathrm{H}_{2} \mathrm{O}_{2}\right)$ and the protection against protein oxidation by PE was examined. The Oxyblot protein oxidation detection kit, an assay that allows detection of carbonyl groups introduced into proteins by oxidation, was used to measure the levels of oxidized proteins. Oxyblot immunoblotting revealed a significant 4-folds increase in the levels of oxidized proteins upon $4 \mathrm{mM} \mathrm{H}_{2} \mathrm{O}_{2}$ treatment for $16 \mathrm{~h}$ (Fig. 4.10a). This is consistent with the notion that $\mathrm{H}_{2} \mathrm{O}_{2}$ is a major reactive oxygen species (ROS) that causes protein oxidation and damage [420]. However, additions of 150 and $300 \mu \mathrm{g} / \mathrm{ml}$ PE markedly reduced the levels of oxidized protein induced by $4 \mathrm{mM} \mathrm{H}_{2} \mathrm{O}_{2}$ (Fig. 4.10a). Concomitant Trypan Blue cell viability assay also showed that both concentrations of PE mitigated the $60 \%$ cell death caused by $\mathrm{H}_{2} \mathrm{O}_{2}$ oxidative stress (Fig. 4.10b). These results demonstrate that PE protects against $\mathrm{H}_{2} \mathrm{O}_{2}$-induced proteotoxicity.

PE harbors antioxidant properties [421], which might account for the reduced protein oxidation during $\mathrm{H}_{2} \mathrm{O}_{2}$ treatment. Hence, to delineate whether the positive effect of PE on proteotoxicity is dependent on autophagy, we blocked the lysosomal activity via NL 
treatment and examined the effects of $\mathrm{PE}$ on $\mathrm{H}_{2} \mathrm{O}_{2}$-induced oxidative injuries. Surprisingly, blockade of lysosomal function did not abolish PE's protection against $\mathrm{H}_{2} \mathrm{O}_{2}$ induced protein oxidation and cell death (Fig. 4.10a and b). Together, our results demonstrate that autophagy induction by PE does not contribute to protection against oxidative stress-induced proteotoxicity and cell death. 
a
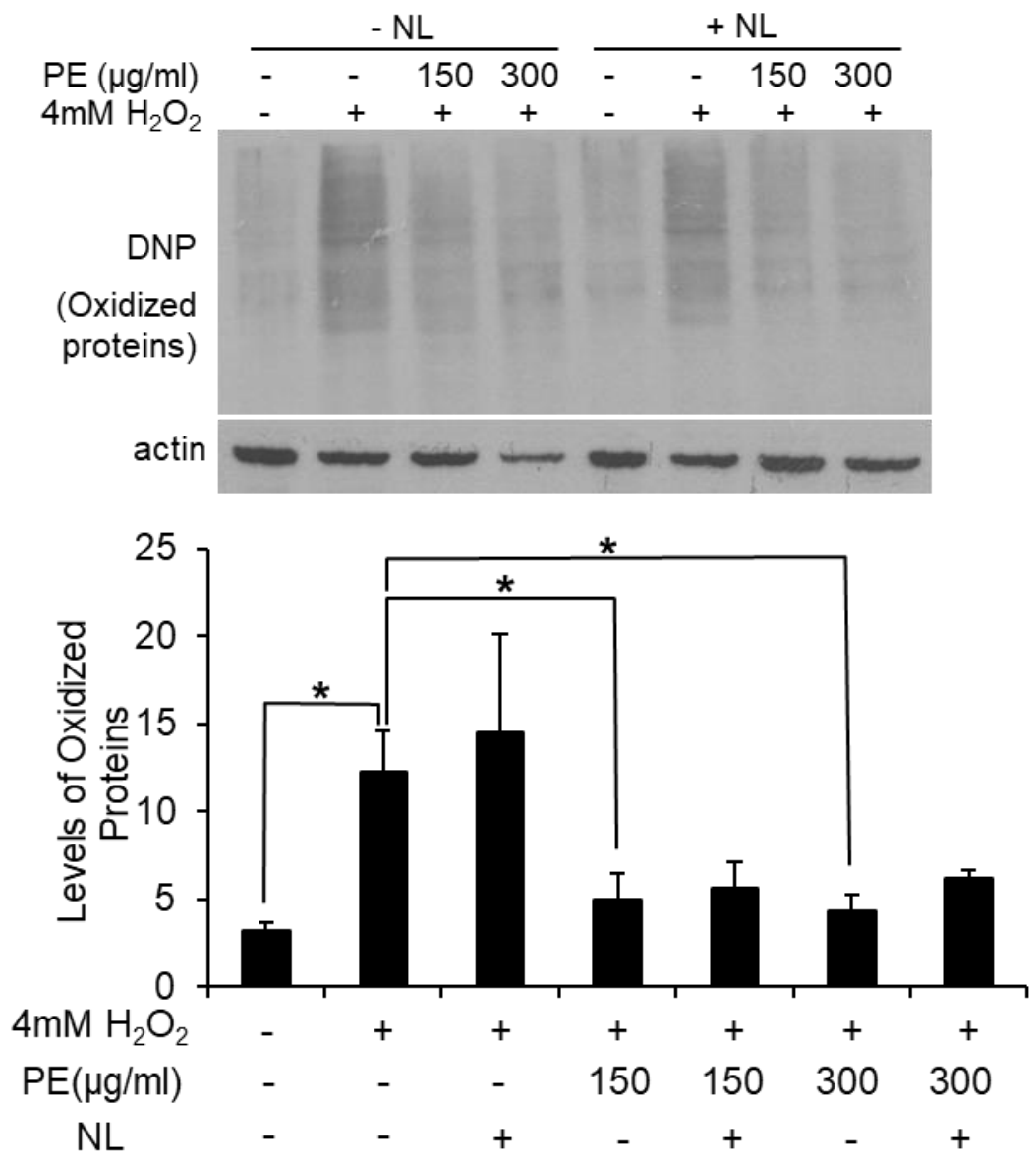

b

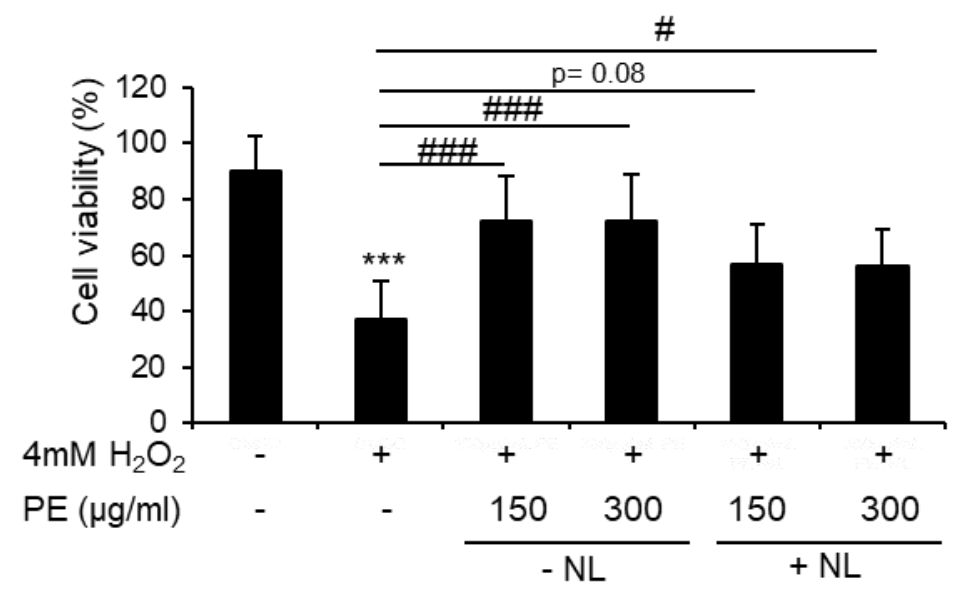


Figure 4.10 PE protects against $\mathrm{H}_{2} \mathrm{O}_{2}$ oxidative stress-induced protein oxidation and cell death independent of autophagy pathway. (a) Top: Oxyblot detection of carbonylated (oxidized) proteins labeled by DNP in SY5Y cells treated according to the indicated conditions for $16 \mathrm{~h}$ in the absence or presence of lysosomal inhibitor (NL). Bottom: Quantification of the levels of carbonylated proteins in various conditions. (b) Cell viability measurement of the corresponding cells treated under various conditions using Trypan Blue assay. A minimum of 300 cells were analyzed for cell viability per experimental set. Values are represented as mean + S.E.M $(n=3-5)$. Differences with no $\mathrm{H}_{2} \mathrm{O}_{2}$ treatment or $\mathrm{H}_{2} \mathrm{O}_{2}$ treatment alone are significant for ${ }^{* *} p<0.01$, ${ }^{* * *} p<0.0005$ and \# $p<0.05, \# \# p<0.01$, \#\# $p<0.0005$ respectively. 


\subsubsection{PE alleviates aggregation-induced toxicity by reducing formation of protein aggregates}

Protein aggregation is a common molecular mechanism underlying the pathogenesis of neurodegenerative diseases [29, 140]. Aggrephagy, or autophagic clearance of protein aggregates, protects the intracellular environment against aggregationinduced toxicity [11]. Here, we further examined whether PE promotes aggrephagy to alleviate proteotoxicity. To do this, we adopted the Synphilin-1 (Sph1) inclusion clearance paradigm described in the previous chapter to evaluate whether PE mediates clearance of Sph1 protein inclusions formed under proteasomal stress. For this experimental setup, we did not discriminate between the different types of inclusions formed by Sph1 (the Agg and Agm), but classified the appearance of any types as Sph1 inclusions.

GFP-Sph1 was overexpressed in SY5Y cells and subsequently subjected to proteasome inhibition with $2 \mu \mathrm{M}$ MG132 to facilitate formation of Sph1 inclusions. With this preformed pool of Sph1 inclusions, the cells were maintained in either DMSO or $300 \mu \mathrm{g} / \mathrm{ml} \mathrm{PE}$ with or without autophagy inhibitor 3-MA. Addition of PE significantly reduced the percentage of cells containing Sph 1 inclusions by approximately $10 \%$ as compared to DMSO-treated control cells (Fig. 4.11). However, the loss of functional autophagy did not abrogate the decrease in percentage of cells harboring Sph1 inclusions under PE treatment (Fig. 4.11). Thus, similar to oxidative stress, the ability of $P E$ to protect the cells against protein aggregation is also not dependent on the autophagy pathway. We proposed that PE may maintain proteostasis either via its antioxidant capacity, or through the upregulation of other homeostatic processes such as the chaperone response to prevent protein aggregation. 

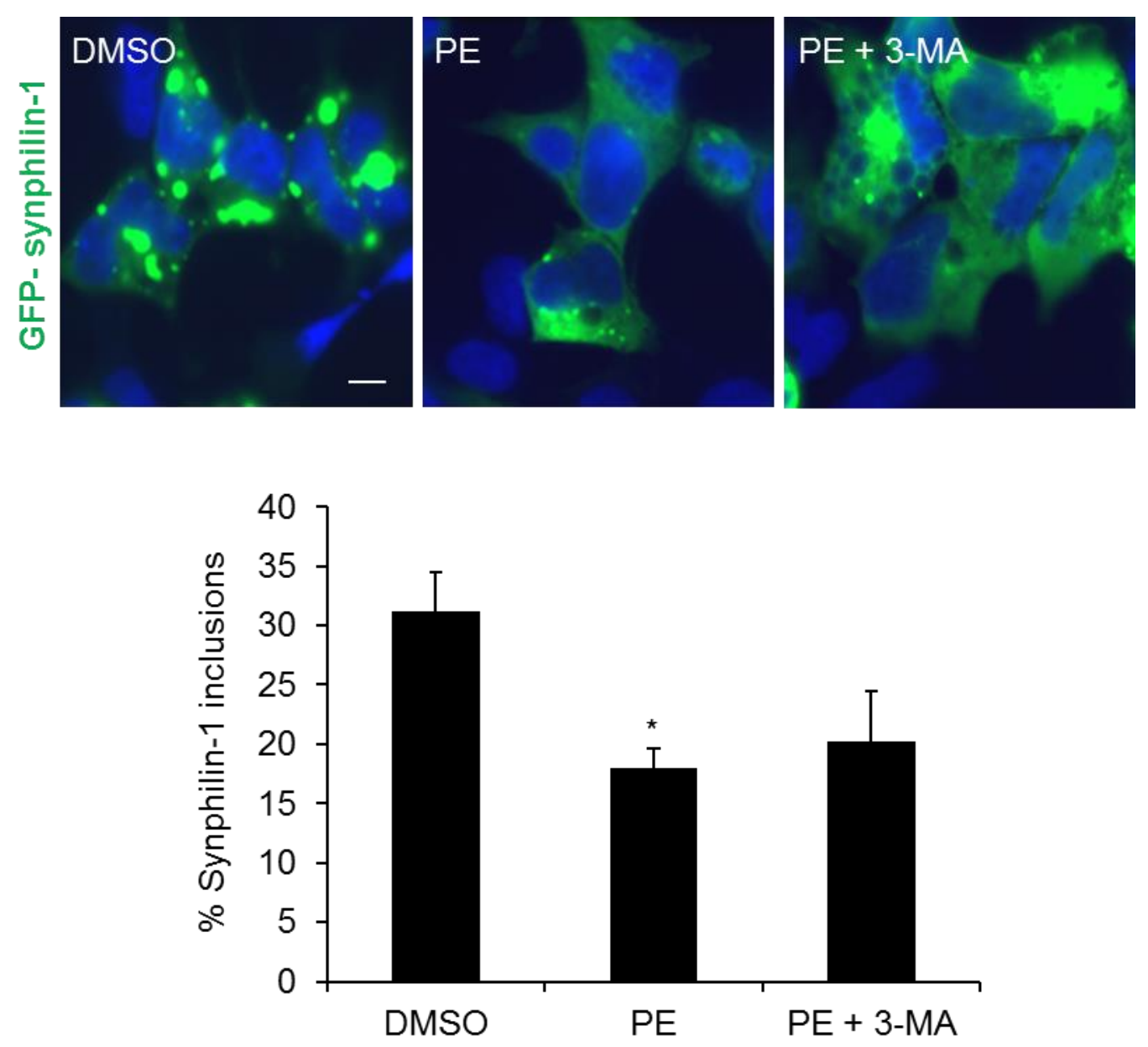

Figure 4.11 PE inhibits protein aggregation of GFP-Sph1 under proteasomal stress. Top: Fluorescence images of Sph1 protein inclusions in SY5Y cells transfected with GFP-Sph1 upon proteasomal inhibition followed by 24h incubation in DMSO or $300 \mu \mathrm{g} / \mathrm{ml}$ PE with or without 3-MA. Nuclei were stained with DAPI. Bottom: Quantification of the percentage Sph1 inclusions in at least 100 transfected cells per condition. All values are mean + S.E.M $(n=3)$. Difference with DMSO is significant for ${ }^{*} p<0.05$. Scale bar, $10 \mu \mathrm{m}$. 


\subsection{PE PROMOTES AUTOPHAGIC CLEARANCE OF DAMAGED MITOCHONDRIA IN RESPONSE TO MITOCHONDRIAL DYSFUNCTION AND MITIGATES MITOCHONDRIAL REDOX TOXICITY}

Clearance of damaged mitochondria by the autophagy pathway (mitophagy) represents another important process for the maintenance of cellular homeostasis [422]. Mitochondria are essential intracellular energy generator to support cellular activities and hence, preservation of mitochondria function as well as homeostasis are critical for eukaryotic cell physiology [275, 422]. Recently, the ROS generated by mitochondrial uncoupler carbonyl cyanide m-chlorophenyl hydrazone (CCCP) was shown to stimulate mitophagy by inducing lysosomal- $\mathrm{Ca}^{2+}$ release from mucolipin-1, a lysosomal calcium channel to activate TFEB through calcineurin-mediated dephosphorylation [423, 424]. Coincidentally, our earlier observations also show that PE upregulates TFEB signaling and autophagy via the lysosomal- $\mathrm{Ca}^{2+}$ signaling. This prompted us to examine the role of PE-TFEB-induced autophagy in mitochondrial quality control.

\subsubsection{CCCP promotes TFEB activation via lysosomal-Ca2+ signaling pathway}

Before evaluating whether PE influences turnover of damaged mitochondria, we examined the effects of CCCP on TFEB activation in SY5Y cells. Treatment of GFPTFEB stable cell line with $10 \mu \mathrm{M}$ CCCP for $24 \mathrm{~h}$ resulted in marked increase in TFEB nuclear localized cells from negligible to approximately 20\% (Fig. 4.12a), an observation in line with the reported data [425]. Interestingly, 300 $\mathrm{g} / \mathrm{ml}$ PE treatment displayed 4 times more cells with TFEB nuclear localization (approximately 75\%) than CCCP, indicating PE is a more potent activator of TFEB than CCCP (Fig. 4.12a). PTFEB $^{S 142}$ immunoblotting revealed that $10 \mu \mathrm{M}$ CCCP treatment for $6 \mathrm{~h}$ and $24 \mathrm{~h}$ significantly reduced P-TFEB ${ }^{S 142}$ levels at both time points (Fig. 4.12b). We next assessed whether the CCCP-induced TFEB activation is dependent on lysosomal$\mathrm{Ca}^{2+}$ signaling. Inhibition of the lysosomal-Ca ${ }^{2+}$ signaling with $10 \mu \mathrm{M}$ FK506 reduced the percentage of cells containing nuclear TFEB from approximately $9 \%$ to $5 \%$ under CCCP condition (Fig. 4.12c). Hence, our result shows that CCCP can also activate TFEB via lysosomal-Ca ${ }^{2+}$ signaling in SY5Y cells. 
a
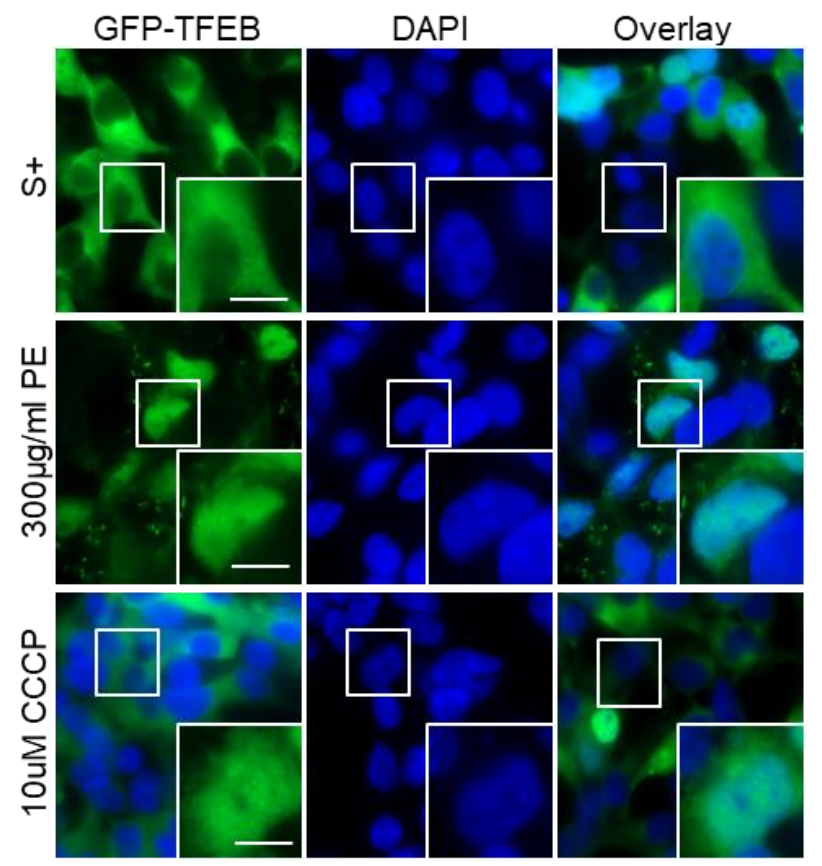

b
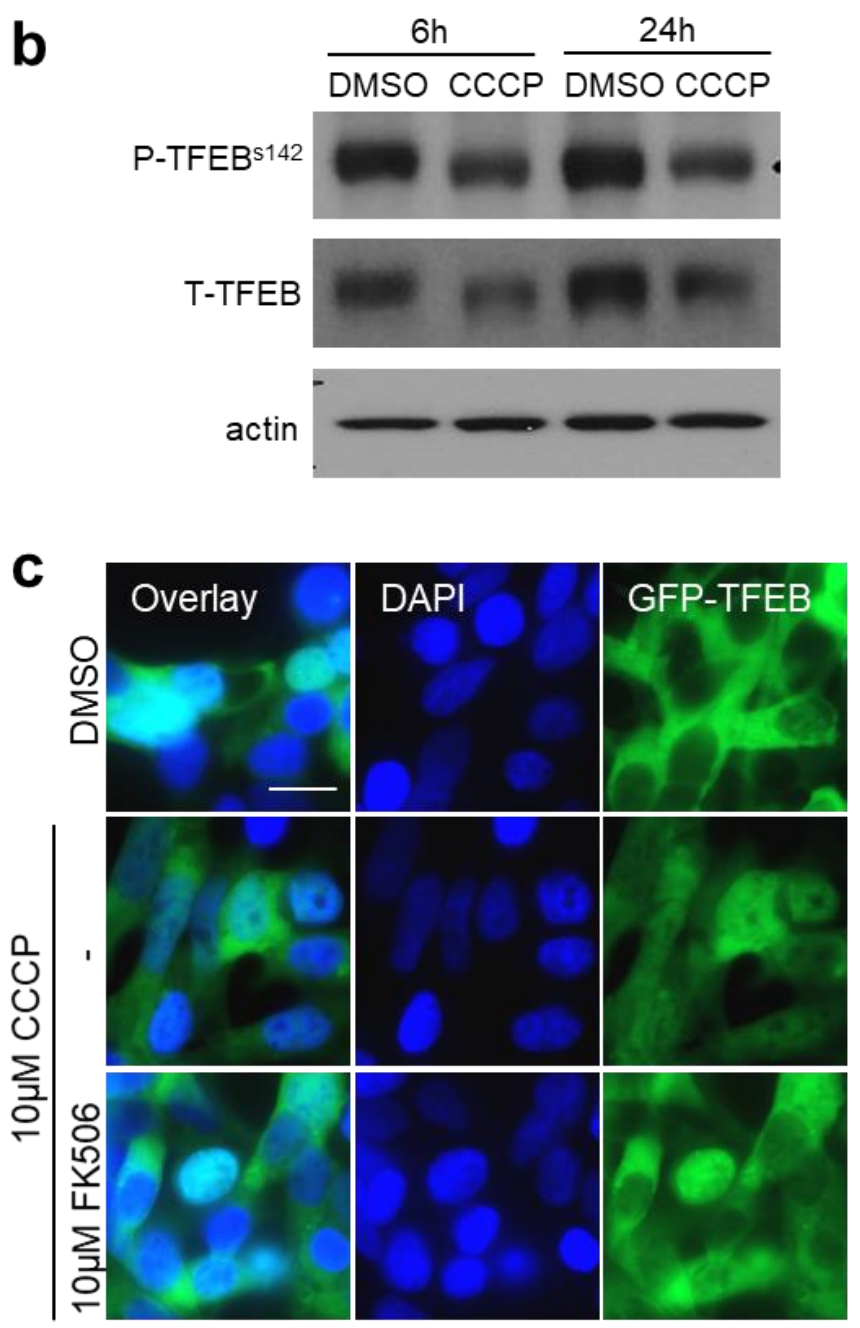
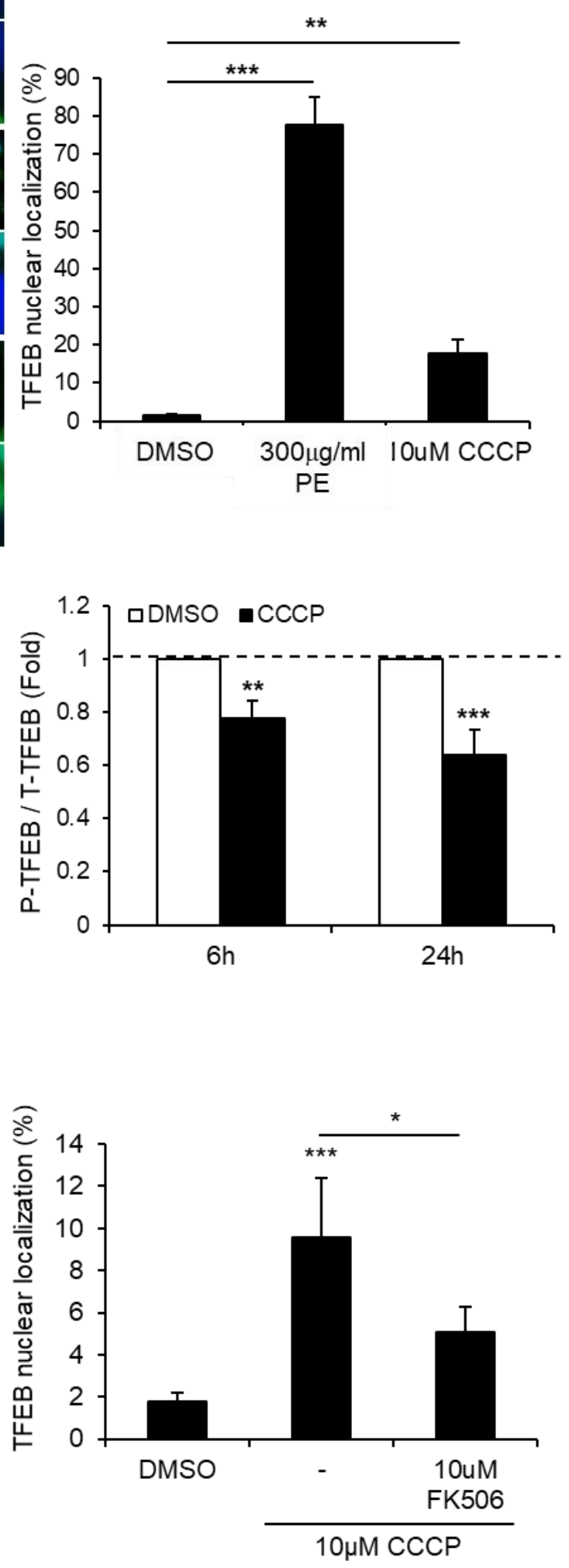
Figure 4.12 CCCP induces TFEB nuclear localization via lysosomal$\mathrm{Ca}^{2+}$ calcineurin signaling. (a) Fluorescence images (left) and quantification of percentage TFEB nuclear localization (right) in GFP-TFEB SY5Y stable cells treated with DMSO, $300 \mu \mathrm{g} / \mathrm{ml}$ PE or $10 \mu \mathrm{M}$ CCCP for $24 \mathrm{~h}$. Smaller inlets show close-up views of GFP-TFEB in selected representative cells under various conditions. A least 100 transfected cells were quantified per condition. Nuclei were stained with DAPI. (b) Left: Immunoblots of P-TFEB ${ }^{\mathrm{S} 142}$ and T-TFEB in GFP-TFEB SY5Y stable cells treated with DMSO or $10 \mu \mathrm{M} \mathrm{CCCP} \mathrm{for} 6$ and 24h. Right: Quantification of P-TFEB ${ }^{\mathrm{S} 142}$ levels against T-TFEB after actin normalization. (c) Fluorescence images (left) and quantification of percentage TFEB nuclear localization (right) in GFP-TFEB SY5Y

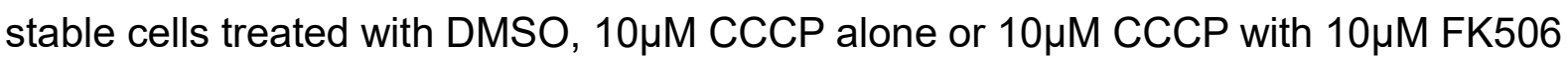
for $24 \mathrm{~h}$. A least 100 cells were quantified per condition. All values are mean + S.E.M $(n=3-4)$. Differences against $S+$, DMSO or CCCP are significant for * $p<0.05$, ** $p<0.01,{ }^{* * *} p<0.0005$. Scale bar, $10 \mu \mathrm{m}$. 


\subsubsection{PE alters mitochondrial morphology}

Mitochondria are highly dynamic organelles that exhibit various types of morphologies influenced by the fission and fusion processes. Mitochondrial dynamics and morphologies play an important role in the preservation of healthy mitochondria and elimination of damaged mitochondria- healthy mitochondria undergo fusion to generate interconnected structures, while the damaged ones undergo fission to segregate themselves from the healthy mitochondria pool for clearance by mitophagy [232, 233]. Hence, the mitochondrial morphology is an indicator of mitochondria health.

A diversity of mitochondrial rearrangements has been observed in the eukaryotic cells, and they can be broadly classified into (1) rod-like/tubular structures, (2) interconnected network, and (3) fragmented structures. The first two types of mitochondrial organization are often associated with healthy mitochondria under physiological condition. In the presence of mitochondrial stress, mitochondrial fragmentation occurs, a phenotypic arrangement that highlights dysfunctional mitochondria (Fig. 4.13a) [232].

To investigate whether PE influences the mitochondrial health, the effects of PE on the mitochondrial morphology in SY5Y cells were examined. Mitochondria were stained with anti-TOMM20, a mitochondrial membrane protein marker, and imaged using a 63x magnification on a live-cell imaging fluorescence microscope. Mitochondria typically appeared as tubular structure or interconnected network under DMSO condition (Fig. 4.13b). In contrast, 16h CCCP treatment led to significant fragmentation of the mitochondria network, a phenotype which reflects mitochondrial dysfunction. Interestingly, 16h treatment with 150 and $300 \mu \mathrm{g} / \mathrm{ml} \mathrm{PE}$ led to formation of characteristic "donut-shaped" mitochondria, which became apparent with $300 \mu \mathrm{g} / \mathrm{ml}$ PE (Fig. 4.13b).

The donut-shaped mitochondria have been reported but the mechanism and functional significance of the donut formation remains elusive. Studies have shown that the donut formation is a transition state under mitochondrial stress whereby the mitochondria 
can revert back to the healthy, tubular morphology if favorable condition returns, or irreversibly proceed to fragmented state if the stress persists [426]. Hence, the donutshaped morphology seems to be an intermediate state that primes the damaged mitochondria for fragmentation and subsequent removal. We proposed that PE may protect the mitochondrial health by keeping the mitochondria in a primed state to accelerate fragmentation and degradation during onset of mitochondrial stress. 
a

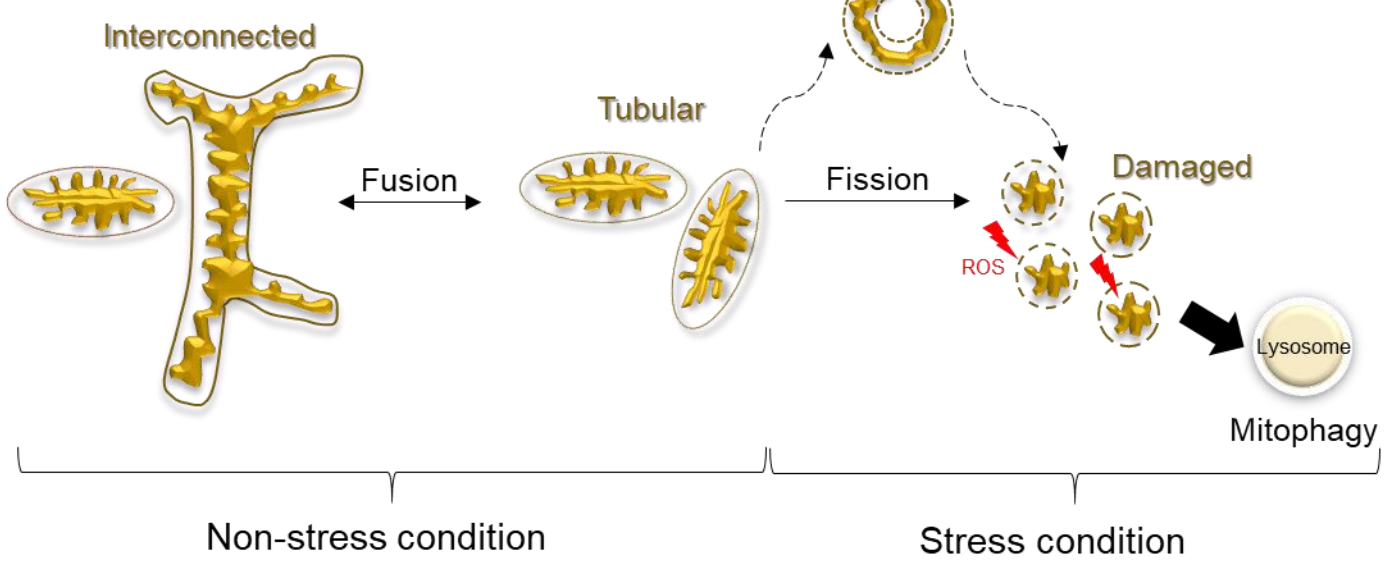

b

Inter
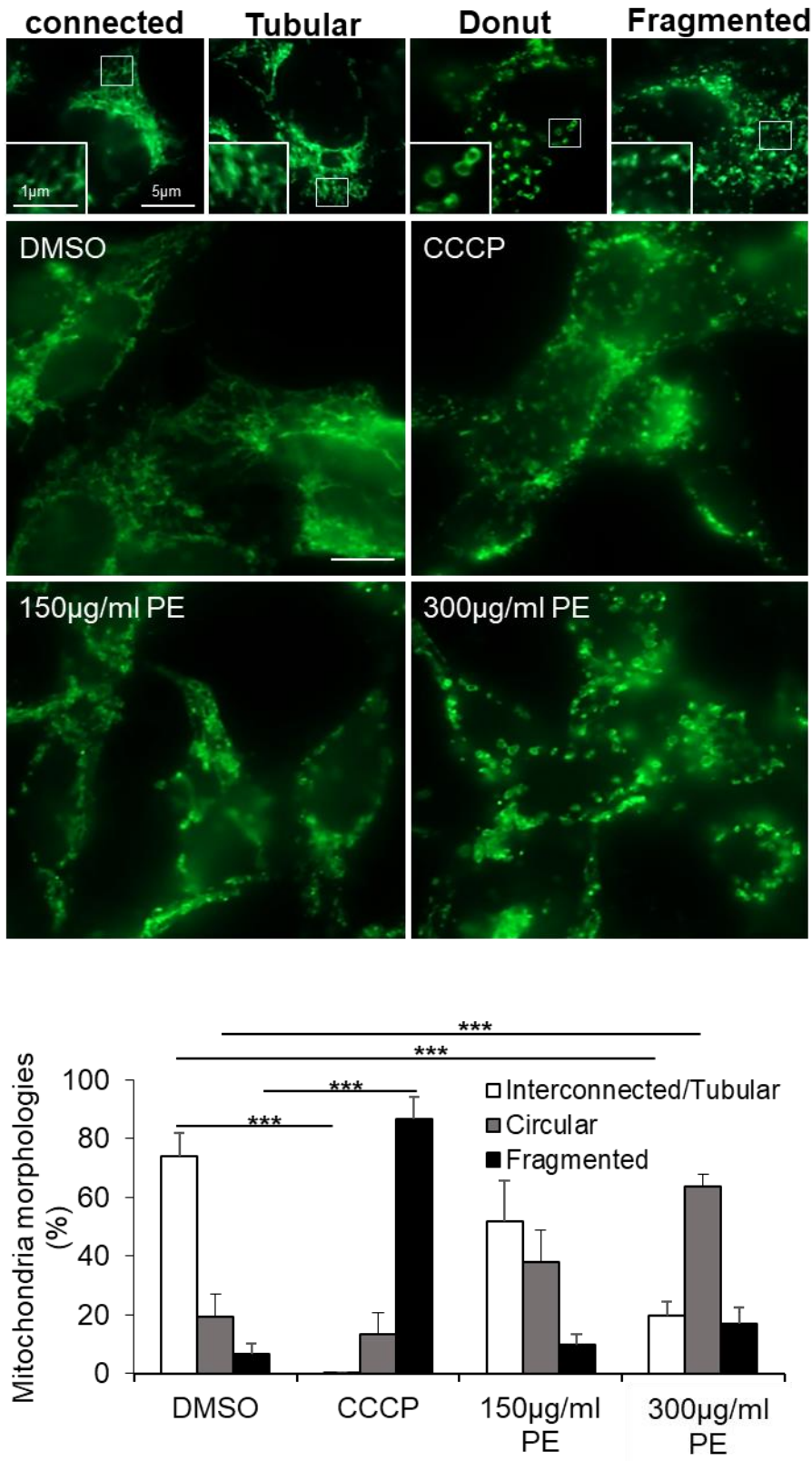
Figure 4.13 PE alters mitochondrial morphology. (a) Illustration of the mitochondrial conformational dynamics under non-stress cellular condition and upon exposure to cellular stressor. (b) Top: anti-TOMM20 immunofluorescence staining

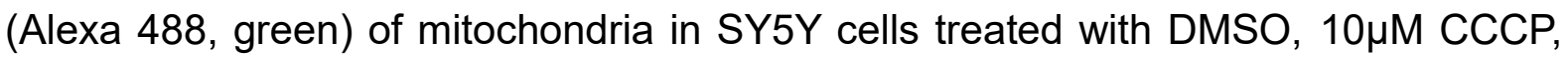
150 or $300 \mu \mathrm{g} / \mathrm{ml} \mathrm{PE}$ for $16 \mathrm{~h}$. Representative views of the different types of mitochondrial morphology are shown in the smaller inlets below. Bottom: Quantification of the various mitochondria morphology observed in the above treated cells. At least 30 cells per condition were analyzed. Values are represented as mean + S.E.M $(n=4)$. Differences against DMSO are significant for ${ }^{* * *} p<0.005$. 


\subsubsection{PE promotes recruitment of the autophagosomes to the mitochondria basally and under CCCP-induced mitochondrial stress}

With the observation that PE alters mitochondrial morphology, we were keen to examine how this morphological change might modulate the interactions between the mitochondria and autophagosomes under basal and CCCP-induced stress conditions. SY5Y cells were co-stained with anti-TOMM20 and LC3 to analyze the colocalization between the two markers under $\mathrm{S}+$ and $10 \mu \mathrm{M}$ CCCP treatments.

Under S+ condition, 150 and $300 \mu \mathrm{g} / \mathrm{ml}$ PE significantly induced colocalization between TOMM20 and LC3 by 1 to 1.5 -folds as compared to DMSO-treated cells (Fig. 4.14a). Specifically, the LC3 puncta localized to and decorated the "ring" of the donut-shaped mitochondria, which became apparent with $300 \mu \mathrm{g} / \mathrm{ml}$ PE. Upon CCCP-induced stress, approximately 1 -fold enhancement in the colocalization between TOMM20 and LC3 was observed as compared to DMSO-treated cells, in agreement with the reported study [427]. Interestingly, additions of 150 and 300 $\mathrm{g} / \mathrm{ml}$ PE under CCCP-induced stress further enhanced the colocalization between both markers (Fig. 4.14a). These observations highlight that PE promotes recruitment of the autophagosomes to mitochondria under physiological and mitochondrial stress conditions.

To evaluate if the enhanced association between the mitochondria and autophagosomes induced by PE under basal and CCCP conditions were dependent on autophagy, we examined the magnitude of mitophagosome accumulation upon autophagy inhibition with $1 \mu \mathrm{M}$ vinblastine $(\mathrm{Vb})$, a microtubule-disruptive drug that inhibits the fusion between autophagosome and lysosome. Under basal condition, $300 \mu \mathrm{g} / \mathrm{ml}$ PE consistently increased the colocalization between TOMM20 and LC3 (Fig. 4.14b). However, no further increase in colocalization was observed between both organelles upon $\mathrm{Vb}$ treatment. In contrast, addition of $\mathrm{Vb}$ led to approximately a marked 0.5 to 1 -fold accumulation of mitophagosomes in the presence of PE under CCCP stress (Fig. 4.14b). Biochemical analysis of the levels of LC3-II associated with the purified mitochondria also indicated an overt increase in the LC3-II marker in the mitochondrial fractions upon PE treatment basally (Fig. 4.14c) and under CCCPinduced stress (Fig. 4.14d). The levels of LC3-II marker further increased in 
mitochondrial fractions upon lysosomal inhibition under both conditions (Fig. $4.14 \mathrm{C}$ and d). This suggests a possible turnover of mitochondria by PE-induced autophagy under both conditions.

Collectively, these results demonstrate two important roles of PE in basal and stressinduced mitochondrial quality control. First, PE promotes recruitment of autophagosomes to the mitochondria basally and may facilitate basal mitophagy as shown by biochemical analysis of purified mitochondria, although IF analysis was not sensitive to detect this clearance. Secondly, the enhanced autophagosome recruitment to the mitochondria upon PE treatment during CCCP-induced stress could lead to higher mitophagy. This is because the loss of intact autophagy led to further accumulation of the two compartments under CCCP stress. Hence, while PE potentially promotes mitophagy during mitochondrial stress, this phenomenon is not observed physiologically. 
a
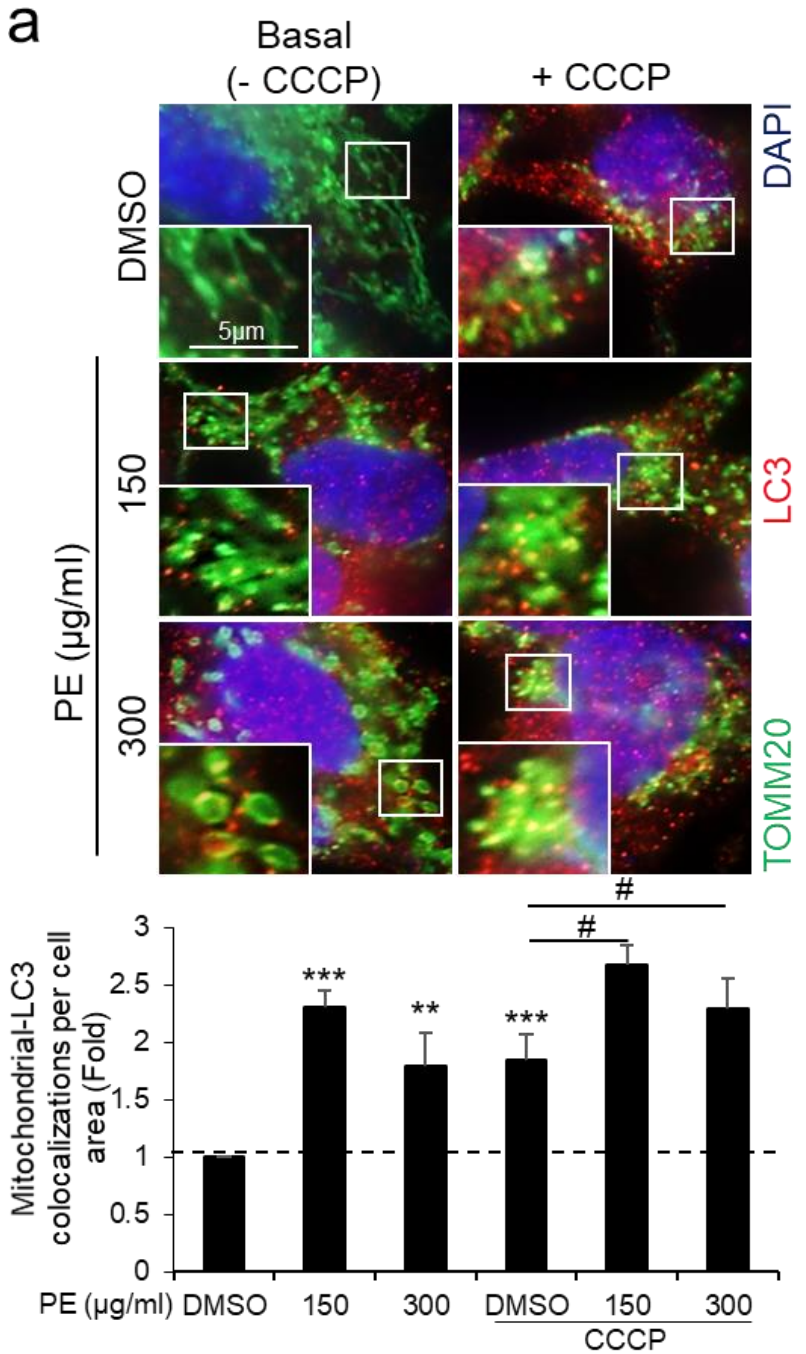

C

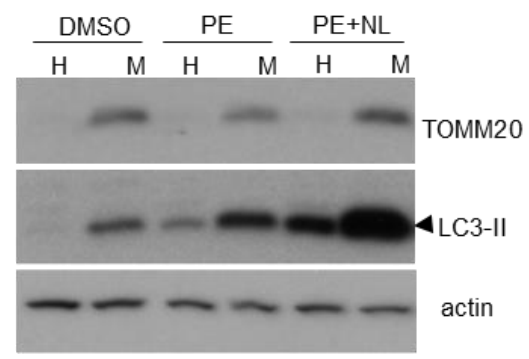

b
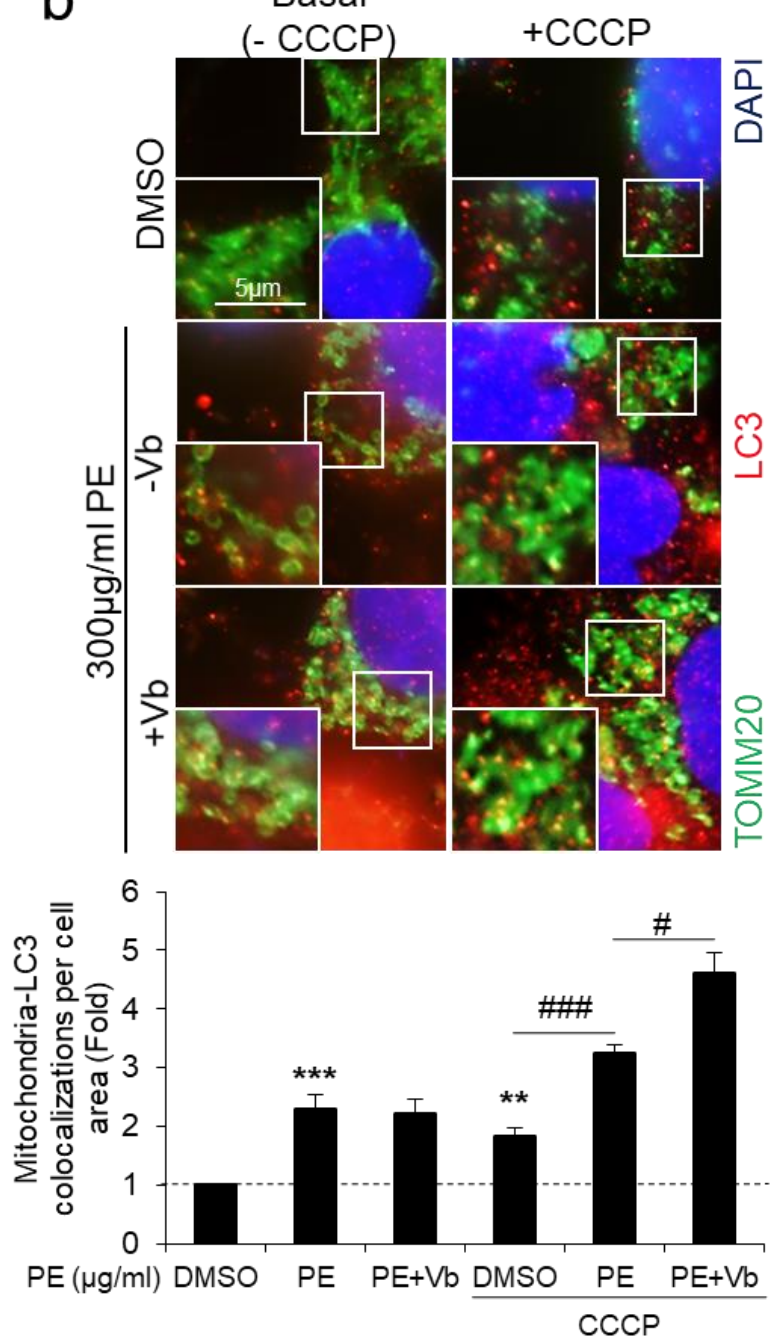

d

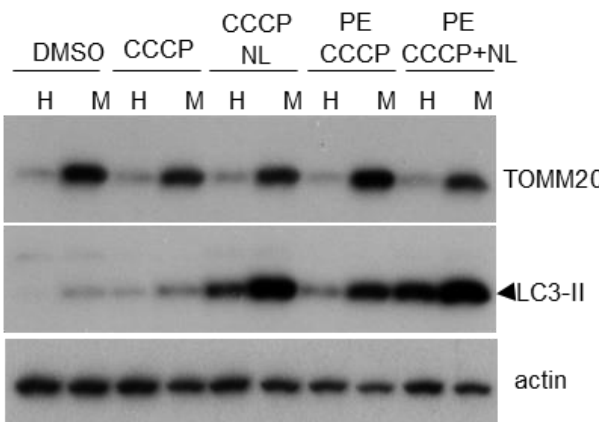


Figure 4.14 PE promotes recruitment of the autophagosomes to the mitochondria under basal and CCCP-induced stress conditions, specifically for turnover of mitochondria during stress. (a) Top: Immunofluorescence staining of anti-TOMM20 (Alexa 488, green) and anti-LC3 (Alexa 555, red) in SY5Y cells treated with DMSO, 150 or $300 \mu \mathrm{g} / \mathrm{ml} \mathrm{PE}$ in the absence or presence of $10 \mu \mathrm{M} \mathrm{CCCP}$ for $16 \mathrm{~h}$. Smaller inlets show close-up views of colocalization between TOMM20 and LC3. Bottom: Quantification of colocalization between TOMM20 and LC 3 in at least 30 cells per condition. (b) Top: Effects of disrupting autophagosome-lysosome fusion with $1 \mu \mathrm{M}$ vinblastine (Vb) on TOMM2O and LC3 colocalization in SY5Y cells treated with DMSO or $300 \mu \mathrm{g} / \mathrm{m} \mathrm{PE}$ in the absence or presence of $10 \mu \mathrm{M} \mathrm{CCCP}$ for $16 \mathrm{~h}$. Bottom: Quantification of TOMM20 and LC3 colocalization in at least 30 cells per condition. Nuclei were stained with DAPI. (c) Immunoblots of TOMM20 and LC3 in total cell homogenate $(\mathrm{H})$ and isolated mitochondrial fraction $(\mathrm{M})$ prepared from SY5Y cells treated with DMSO or $300 \mu \mathrm{g} / \mathrm{ml}$ PE for 16h. (d) Immunoblots of TOMM20 and LC3 in total cell homogenate $(\mathrm{H})$ and isolated mitochondrial fraction $(\mathrm{M})$ in SY5Y cells treated with DMSO, $10 \mu \mathrm{M} \mathrm{CCCP}$ alone or with lysosomal inhibitor $(\mathrm{NL})$ and $10 \mu \mathrm{M} \mathrm{CCCP}$ supplemented with $300 \mu \mathrm{g} / \mathrm{ml} \mathrm{PE}$ with or with NL for $16 \mathrm{~h}$. All values are mean + S.E.M $(n=3-7)$. Differences against DMSO or CCCP treatment only are significant for ** $p<0.01,{ }^{* * *} p<0.0005$ and $\# p<0.05, \# \# p<0.0005$ respectively. Scale bar is $10 \mu m$ unless otherwise stated. 


\subsubsection{PE promotes Parkin-PINK1 dependent mitophagy under CCCP-induced mitochondrial stress}

Parkin-PINK1 mediated mitophagy is a well-characterized pathway reported to facilitate clearance of mitochondria under CCCP-induced stress [328, 427-429]. To determine if PE driven mitophagy during CCCP stress involves PINK1 and Parkin, we examined the colocalization of both players with the mitochondria during CCCP stress in the presence of PE. Treatment with 150 and $300 \mu \mathrm{g} / \mathrm{ml}$ PE showed negligible effects on colocalization between PINK1 and TOMM20 basally (Fig. 4.15a), consistent with earlier observation that PE did not influence basal mitophagy. Upon 10 $\mu \mathrm{M}$ CCCP treatment, the presence of PINK1 significantly increased in the mitochondria, which was further enhanced upon co-treatment with 150 and 300 $\mu \mathrm{g} / \mathrm{ml} \mathrm{PE} \mathrm{(Fig.} \mathrm{4.15a).} \mathrm{Thus,}$ PE enhances PINK1 recruitment to the mitochondria under mitochondrial stress.

Next, the involvement of Parkin in PE-induced mitophagy was investigated. Using mCherry-Parkin overexpressing SY5Y stable cells, the colocalization between mitochondria and Parkin was examined. Basally, mCherry-Parkin displayed cytosolic distribution with no apparent colocalization with TOMM20 (Fig. 4.15b). However, 6h CCCP treatment led to the formation of "mitochondrial aggregates" that colocalized with mCherry-Parkin. Interestingly, $300 \mu \mathrm{g} / \mathrm{ml}$ PE supplementation increased the percentage of cells displaying Parkin and TOMM20 colocalization from approximately $30 \%$ to $40 \%$ (Fig. 4.15b). Unlike the earlier experiments that looked at the effects of PE upon 12-24h treatment, we only tracked the effects of PE on TOMM20 and Parkin colocalization upon 6h treatment here as longer CCCP treatment initiated cell death in the mCherry-Parkin stable cells. Nonetheless, PE had been shown in earlier results to upregulate autophagic compartment and TFEB activation as early as $6 \mathrm{~h}$ treatment. Hence, our observation of enhanced colocalization between TOMM20 and Parkin with 6h of PE treatment under CCCP stress remained relevant.

Together, our results demonstrate that PE-induced mitophagy under mitochondrial stress involves the Parkin-PINK1 pathway, as PE enhanced recruitment of both molecular partners to the mitochondria under CCCP stress. 
a
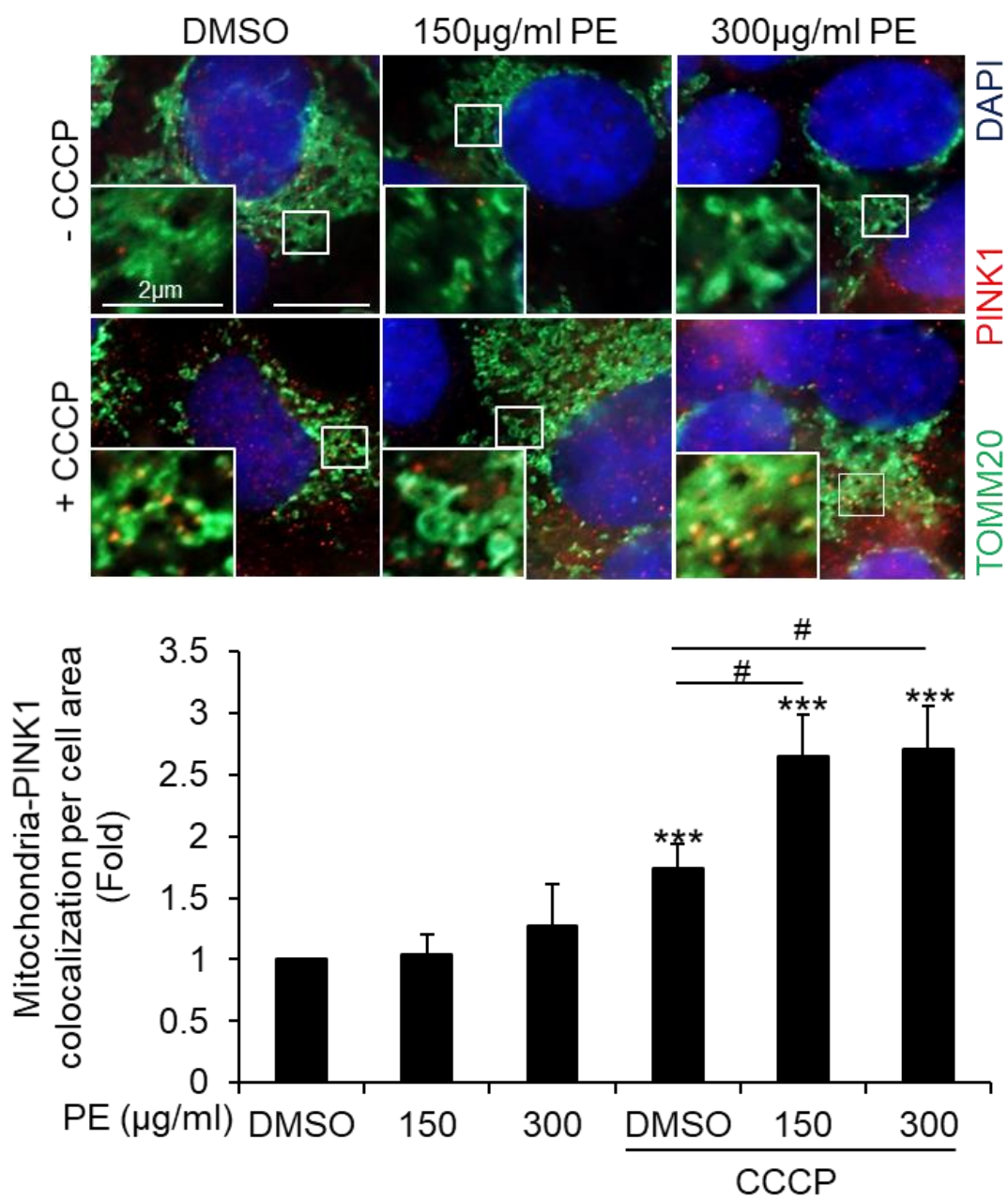

b

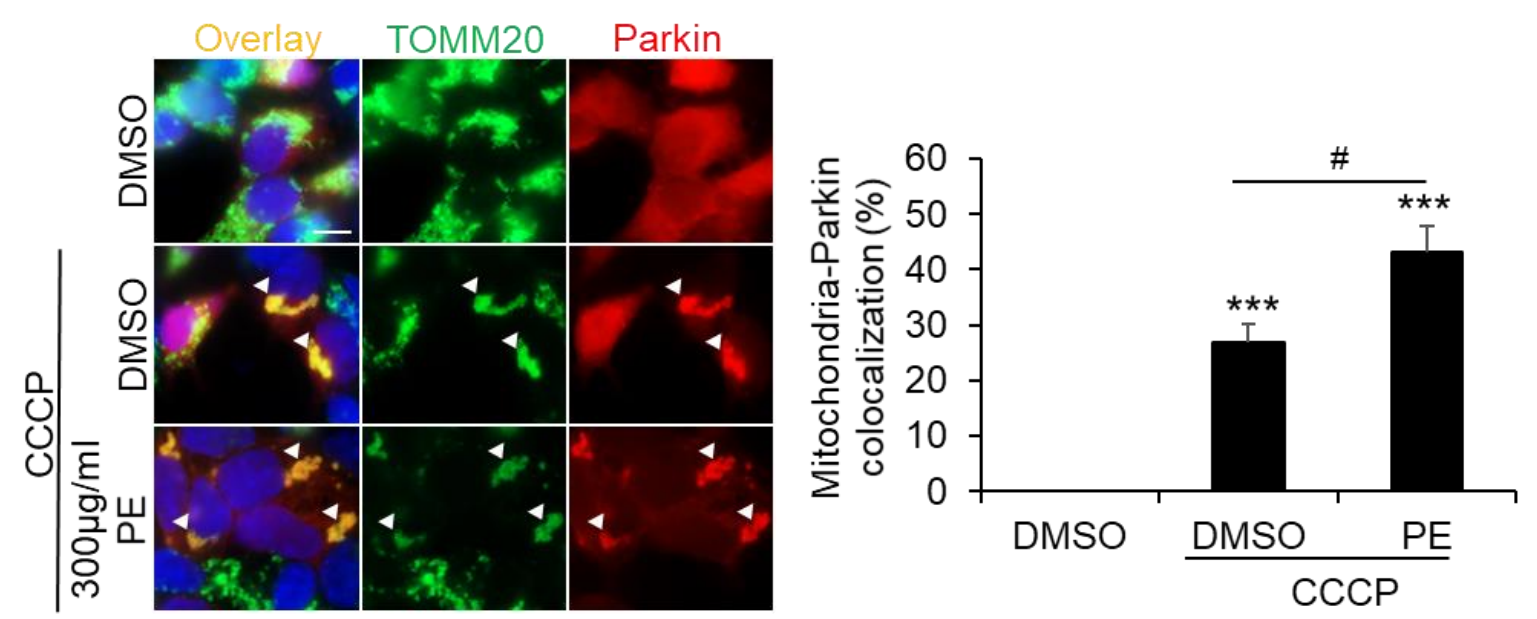


Figure 4.15 PE promotes recruitment of PINK1 and Parkin to mitochondria under CCCP-induced stress. (a) Top: Immunofluorescence staining of TOMM20 (Alexa 488, green) and PINK1 (Alexa 555, red) in SY5Y cells treated with DMSO, 150 or $300 \mu \mathrm{g} / \mathrm{ml} \mathrm{PE}$ in the absence or presence of $10 \mu \mathrm{M} \mathrm{CCCP}$ for $16 \mathrm{~h}$. Smaller inlets show close-up views of TOMM20 and PINK1 colocalization. Bottom: Quantification of TOMM20 and LC3 colocalization per cell area under the respective conditions. At least 30 cells from random fields were analyzed for each condition. Nuclei were stained with DAPI. (b) Left: TOMM20 immunofluorescence staining (Alexa 488, green) in mCherryParkin SY5Y stable cells treated with DMSO or $10 \mu \mathrm{M} \mathrm{CCCP} \mathrm{in} \mathrm{the} \mathrm{absence} \mathrm{or}$ presence of $300 \mu \mathrm{g} / \mathrm{ml}$ PE for $6 \mathrm{~h}$. White arrows depict the colocalization between mitochondrial aggregates and Parkin. Right: Quantification of TOMM20 and Parkin colocalization (arrows) under the respective conditions. At least 30 cells from random fields were analyzed for each condition. All values are mean + S.E.M (n=3-4). Differences against DMSO or CCCP treatment only are significant for * $p<0.05$, ${ }^{* * *}$ $p<0.0005$ and $\# p<0.05$ respectively. Scale bar is $10 \mu \mathrm{m}$ unless otherwise stated. 


\subsubsection{PE promotes degradation of mitochondria under CCCP-induced stress}

To further substantiate that PE augments stress-induced mitophagy, we monitored the effect of PE on the rate of mitochondrial clearance during CCCP stress. We tracked the TOMM20 levels in SY5Y cells, over a 12h CCCP treatment time-course in the absence or presence of $300 \mu \mathrm{g} / \mathrm{m}$ PE via immunoblotting (Fig. 4.16a). CCCP treatment led to a progressive reduction in TOMM20 levels $\mu$ over time, with significant reduction observed at the $12 \mathrm{~h}$ time point. Addition of PE dramatically accelerated the rate of TOMM20 disappearance under CCCP stress, which was apparent as earlier as $6 \mathrm{~h}$ time point (Fig. 4.16a).

To affirm the faster TOMM20 disappearance was caused by enhanced mitophagy stimulated by PE, TOMM20 flux upon autophagy inhibition was measured (Fig. 4.16b). Changes in TOMM20 levels in CCCP-treated SY5Y cells upon autophagic inhibition $(1 \mu \mathrm{M} \mathrm{Vb}$ and $\mathrm{NL})$ were compared in the absence and presence of $300 \mu \mathrm{g} / \mathrm{ml} \mathrm{PE}$. To prevent mitochondrial biogenesis event from interfering with the accumulation of TOMM20 levels, protein synthesis was blocked with $10 \mu \mathrm{M}$ cycloheximide (CHX). Results showed that $10 \mu \mathrm{M} \mathrm{CHX}$ successfully inhibited protein synthesis, as evident from the reduction in TOMM20 levels in the presence of $\mathrm{CHX}$ under CCCP treatment or co-treatment with PE (Fig. 4.16b).

Inhibition of the autophagy pathway led to a slight accumulation of TOMM20 in SY5Y cells treated with CCCP for $12 \mathrm{~h}$ (Fig. 4.16c). In contrast, co-treatment with $300 \mu \mathrm{g} / \mathrm{ml}$ PE induced 50\% more accumulation of TOMM20 upon autophagic inhibition under similar CCCP condition (Fig. 4.16c). Clearly, PE contributes to the quality control of damaged mitochondria during stress, by increasing the rate of mitochondrial autophagic removal. 
a
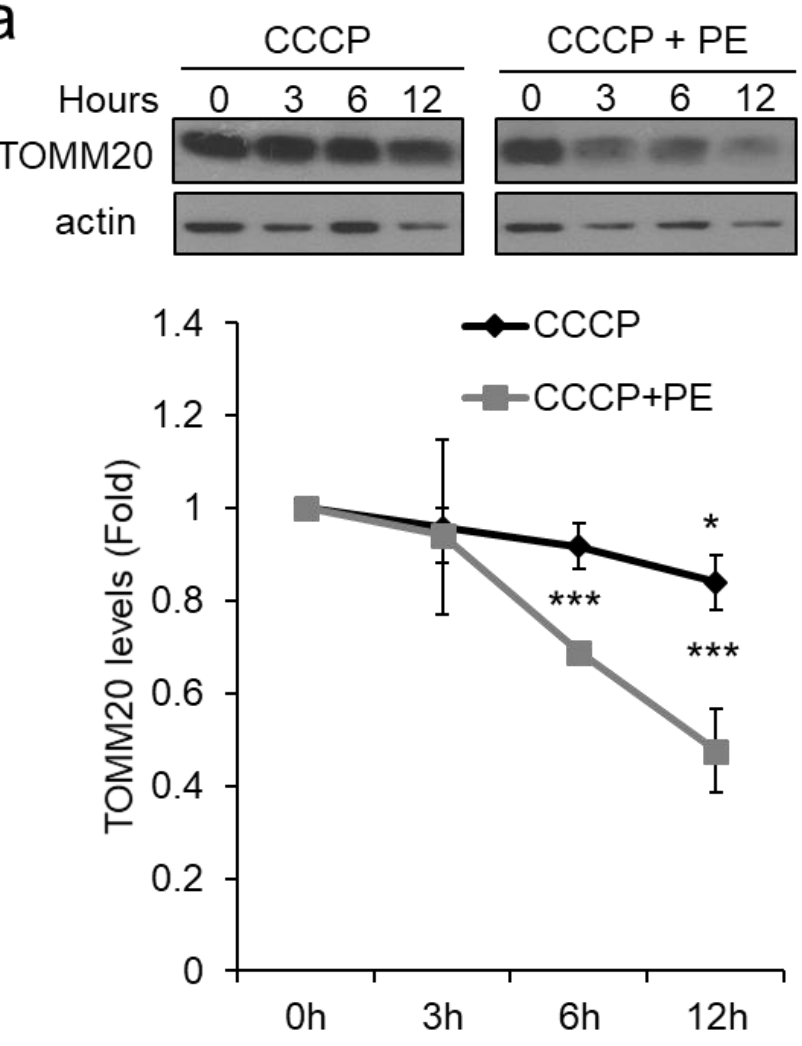

b

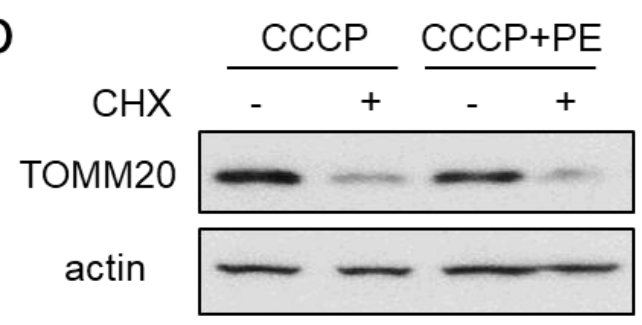

C

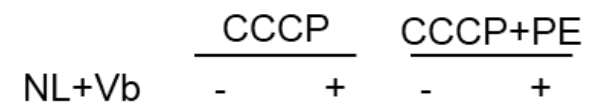

TOMM20
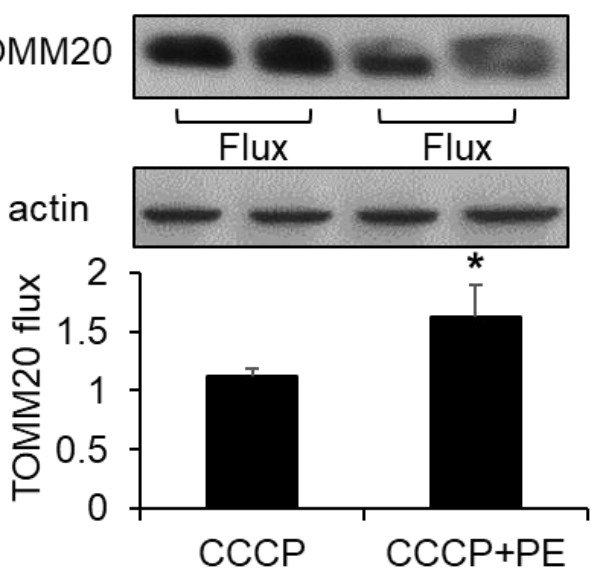

Figure 4.16 PE promotes autophagic clearance of mitochondria (mitophagy) under CCCP-induced stress. (a) Top: Immunoblot of TOMM20 in SY5Y cells over a $12 \mathrm{~h}$ time-course treatment with $10 \mu \mathrm{M} \mathrm{CCCP} \mathrm{in} \mathrm{the} \mathrm{absence} \mathrm{or} \mathrm{presence} \mathrm{of} 300 \mu \mathrm{g} / \mathrm{ml}$ PE. Bottom: Quantification of TOMM20 levels at each time point after normalization against actin, expressed as fold change against $0 \mathrm{~h}$. (b) Immunoblot of TOMM20 in SY5Y cells under CCCP alone or supplemented with $300 \mu \mathrm{g} / \mathrm{ml} \mathrm{PE}$, in the absence or presence of $10 \mu \mathrm{M}$ cycloheximide (CHX) (c) Top: Immunoblot of TOMM20 in SY5Y cells treated with $10 \mu \mathrm{M} \mathrm{CHX}$ and subjected to $10 \mu \mathrm{M} \mathrm{CCCP}$ alone or supplemented with $300 \mu \mathrm{g} / \mathrm{ml} \mathrm{PE}$ in the absence or presence of lysosomal inhibition (NL+Vb: NL with $1 \mu \mathrm{M}$ vinblastine) for $12 \mathrm{~h}$. Bottom: Quantification of CCCP-induced TOMM20 flux in the absence or presence of PE expressed as TOMM20 levels under lysosomal inhibition against untreated condition after normalization against actin. All values are mean + S.E.M ( $n=3-4)$. Differences against DMSO or CCCP treatment only are significant for ${ }^{*} p<0.05$ and ${ }^{* * *} p<0.0005$. 


\subsubsection{PE maintains mitochondrial homeostasis by preventing accumulation of damaged mitochondria and reduces mitochondrial ROS during mitochondrial stress}

Damaged mitochondria are endogenous generators of harmful ROS in the cells [400]. Failure to efficiently remove the dysfunctional mitochondria to prevent their toxic accumulation has shown to enhance ROS production and oxidative cellular damage seen in aging and many age-related diseases [400, 430, 431]. Our results so far demonstrate that PE markedly promotes the recruitment of autophagosomes to the mitochondria under basal and mitochondrial stress conditions (Fig. 14) to facilitate the autophagic removal of mitochondria (Fig. 16). Here, we were interested to investigate the functional significance of PE-mediated mitophagy in maintaining mitochondrial fitness, particularly if the process helps to remove damaged mitochondria to protect against harmful ROS generated by the dysfunctional mitochondria.

To investigate whether PE promotes the turnover of mitochondrial pool nondiscriminately or selectively, targeting only the damaged ones for degradation, we looked at PE effects on MitoTimer, a reporter probe for studying mitochondrial content, age, damage and turnover [381-383]. MitoTimer is a duo-fluorescence protein targeted to the mitochondrial matrix, where its fluorescence shifts irreversibly from green in the newly synthesized healthy mitochondria, to red in damaged mitochondria following oxidation events. To examine how PE influences the levels of damaged mitochondria, we created a SY5Y stable cell line expressing MitoTimer, to examine the green and red fluorescence intensities of the MitoTimer upon treatment with DMSO or $300 \mu \mathrm{g} / \mathrm{ml}$ PE basally or in the presence of CCCP stress. Fluorescence imaging shows that the levels of green MitoTimer proteins remained relatively unchanged across all conditions (Fig. 4.17a), hence suggesting that the pool of newly synthesized, healthy mitochondria was not affected by PE or CCCP stress. On the other hand, the levels of red MitoTimer proteins fluctuated in the various treatment conditions. Basally without CCCP stress, addition of PE caused a slight decrease in the levels of red MitoTimer proteins compared to DMSO control (Fig. 4.17a). Upon CCCP treatment, the levels of red MitoTimer signal significantly increased by $20 \%$ as compared to untreated cells, as expected for the mitochondrial oxidative damage caused by the mitochondrial 
uncoupling drug. Remarkably, addition of PE significantly reduced the levels of red MitoTimer proteins induced by CCCP (Fig. 4.17a). Thus, PE promotes specific handling of dysfunctional mitochondria by autophagy to maintain mitochondrial fitness especially during mitochondrial insult.

Next, we investigated the protectiveness of PE-induced mitophagy against harmful ROS generated by the damaged mitochondria using MitoSox. MitoSox is a probe for measuring the levels of superoxide generated by mitochondria. Under physiological condition, the superoxide anion is generated as a by-product of mitochondrial oxidative phosphorylation. However, under mitochondrial stress that disrupts the NADHcoenzyme $\mathrm{Q}$ oxidoreductase (complex I) in the mitochondrial electron transport chain, generation of superoxide is a pervasive source of toxicity and mitochondrial dysfunction. Hence, measurement of the MitoSox levels serves as an indicator of the amount of ROS generated by damaged mitochondria. Upon CCCP treatment, the levels of red MitoTimer signal significantly increased, as expected for the mitochondrial uncoupling drug (Fig. 4.17b). Remarkably, addition of $300 \mu \mathrm{g} / \mathrm{ml}$ PE significantly reduced the levels of red MitoTimer signal induced by CCCP (Fig. 4.17b). To ascertain that the PE protection against CCCP-induced mitochondrial ROS is dependent on autophagy, lysosome activity was inhibited in CCCP and PE co-treated cells to examine the effect of PE on MitoSox levels. Inhibition of autophagy abolished PE's protection against mitochondrial superoxide generation and led to accumulation of mitochondrial ROS under CCCP stress (Fig. 4.17b). Taken together, PE ameliorates cellular redox toxicity arising from ROS generated by dysfunctional mitochondria by targeting their removal. 
a

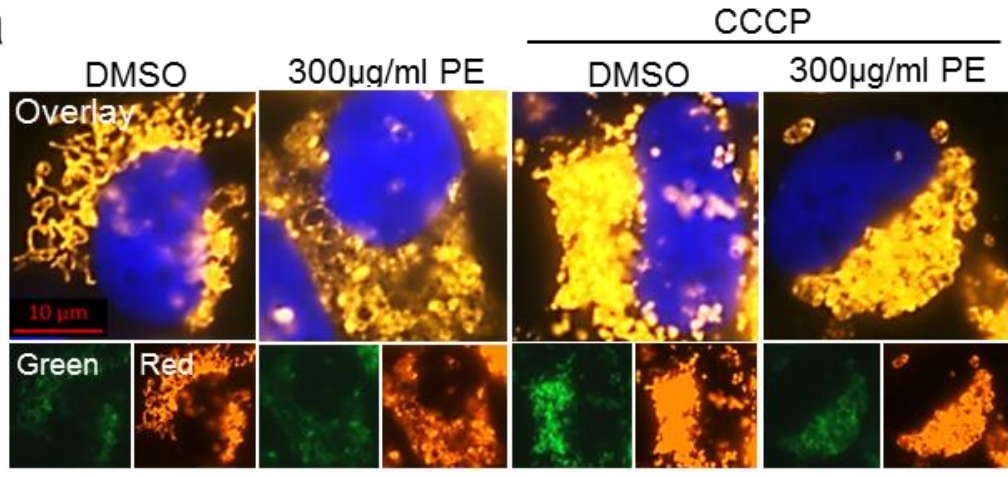

-Green MitoTimer $\square$ "Red" mitotimer

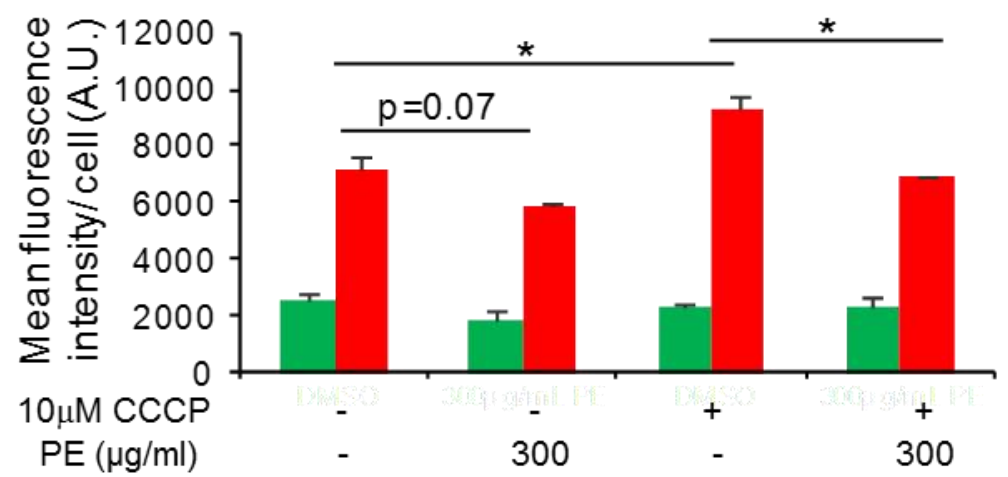

b

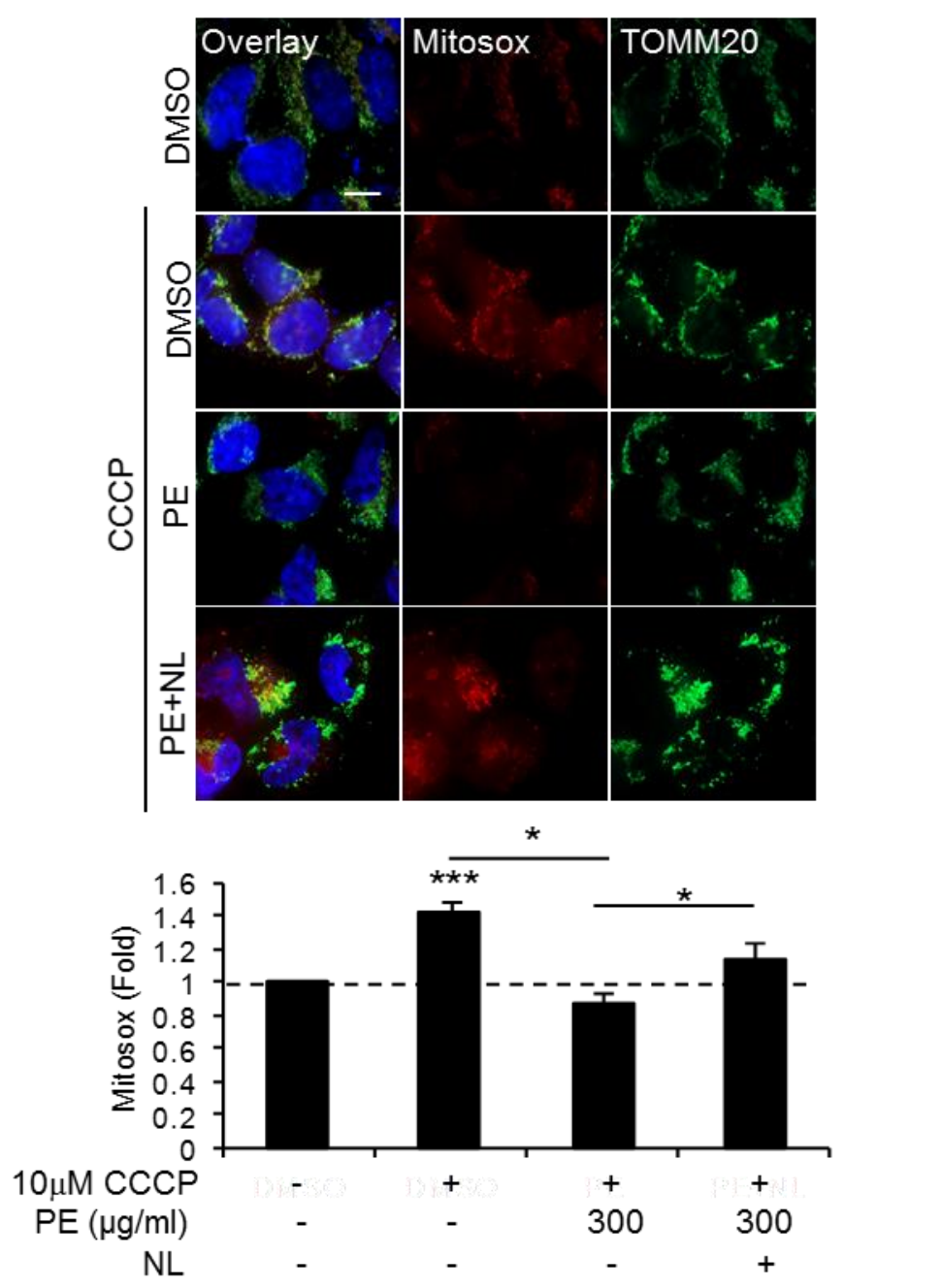




\section{Figure 4.17 PE reduces damaged mitochondria and alleviates mitochondrial} redox toxicity. (a) Measurement of damaged mitochondria using MitoTimer reporter. Top: Fluorescence images of SY5Y cells stably expressing MitoTimer reporter plasmid treated with DMSO or $300 \mu \mathrm{g} / \mathrm{ml} \mathrm{PE}$ in the absence or presence of $10 \mu \mathrm{M} \mathrm{CCCP}$ for 16h. Smaller inlets refer to the green (young mitochondria) and red (aged and damaged mitochondria) MitoTimer expression under each condition. Bottom: Quantification of the green and red intensity of MitoTimer per cell area under the various conditions, expressed as arbitrary unit (A.U). (b) Measurement of mitochondrial ROS via MitoSox staining. Top: TOMM20 and MitoSox

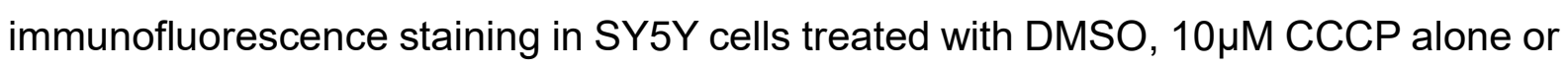
$10 \mu \mathrm{M} \mathrm{CCCP}$ in the presence of $300 \mu \mathrm{g} / \mathrm{m}$ PE or $300 \mu \mathrm{g} / \mathrm{m}$ PE supplemented with lysosomal inhibitors (NL) for 16h. Bottom: Quantification of MitoSox intensity levels per cell area under various conditions expressed as fold change against DMSO only. Nuclei were stained with DAPI. Images were acquired under 63x magnification. At least 30 cells from random fields were analyzed. All values are mean + S.E.M ( $n=3-$ 7). Differences are significant for ${ }^{*} p<0.05,{ }^{* * *} p<0.0005$. Scale bar, $10 \mu \mathrm{m}$. 


\subsection{MAJOR PHYTOCHEMICAL COMPONENTS OF PE REGULATE AUTOPHAGY-LYSOSOMAL FITNESS AND MITOPHAGY-RELATED STRESS RESISTENCE DIFFERENTLY}

The health benefits of pomegranate are associated with the phytochemicals it contains. The most abundant phytochemicals in the pomegranate are the phenolic compounds, which include anthocyanins, hydrolysable tannins, ellagitannins and flavonoids [397, 421]. With the observation that PE upregulates autophagy to elicit a functional benefit on the mitochondrial health by promoting mitophagy under CCCP stress, we were interested to identify the compounds in PE that are responsible for this bioactivity in SY5Y cells. We evaluated the efficacy of the major phenolic compounds enriched in pomegranate, namely ellagic acid (EA), punicalagin (Puni), delphinidin chloride (DC) and luteolin (Lut), in regulating autophagy-lysosomal content and mitophagy. The concentrations tested were based on the dosages reported in published literatures where these compounds have been shown to elicit beneficial effects in cell cultures [411, 432-440].

\subsubsection{PE bioactive components, punicalagin, delphinidin and luteolin, contribute to PE ability to upregulate autophagy via TFEB activation}

All the individual phenolic compounds tested increased the autophagy-lysosomal content but in different manners. Puni, DC and Lut significantly increased the levels of both LAMP1 (lysosomes) and LC3-II (autophagosomes) at the higher concentrations tested, mirroring the effects of PE (Fig. 4.18a and b). Interestingly, EA treatment increased the LC3-II levels without affecting the lysosomal LAMP1 compartment (Fig. 4.18a and b). For TFEB activation, Puni, DC and Lut induced distinct increase in the nuclear translocation of TFEB along with significant reduction in P-TFEBS142 levels, as seen with PE (Fig. 4.18c). However, EA did not have any effect on either phenomenon (Fig. 4.18c). Unanimously, Puni, DC and Lut demonstrate the same ability as PE to enhance autophagy-lysosomal fitness by activating TFEB to increase both the autophagic and lysosomal compartments. Interestingly, EA mainly influences the autophagic compartments in a TFEB independent manner. 
a
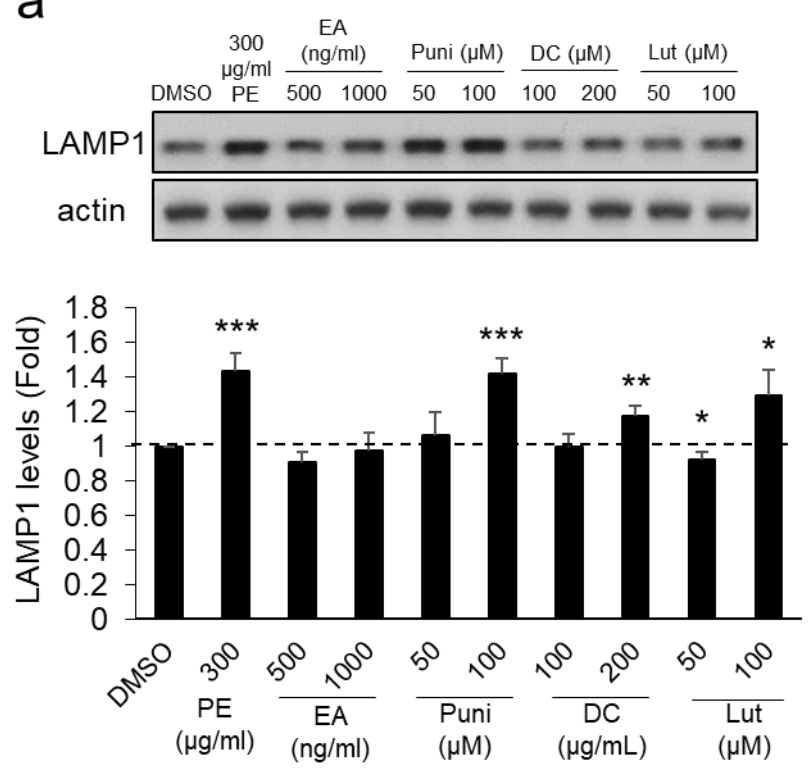

b
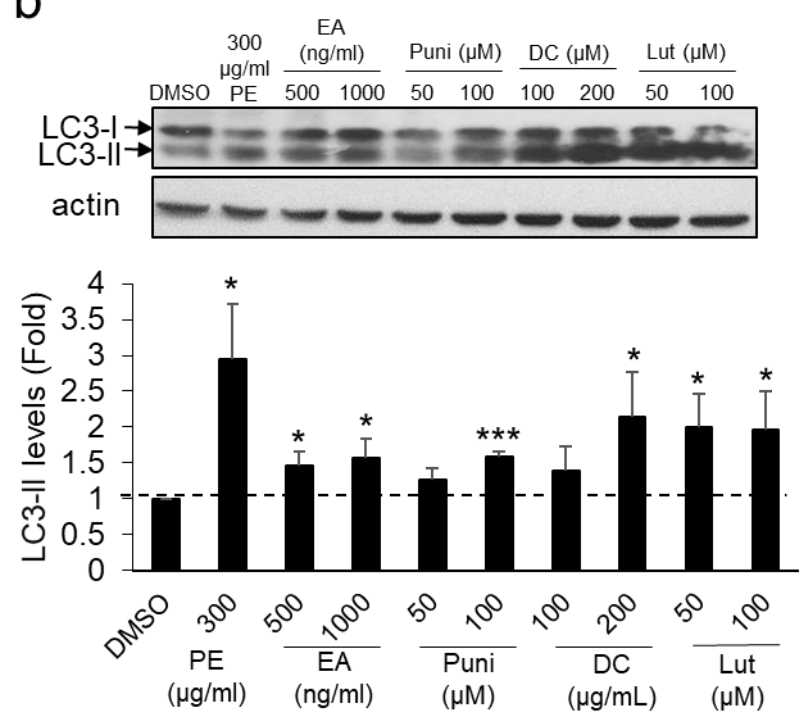

d
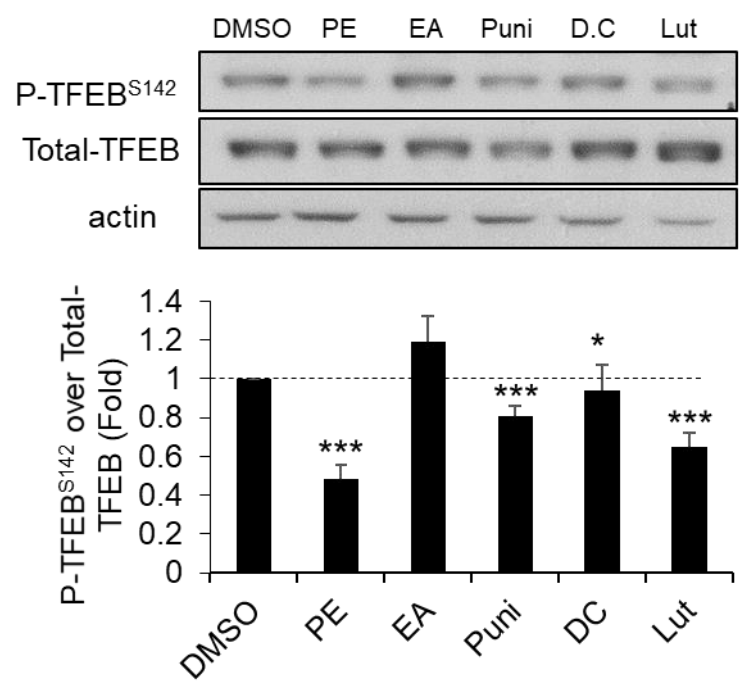

C
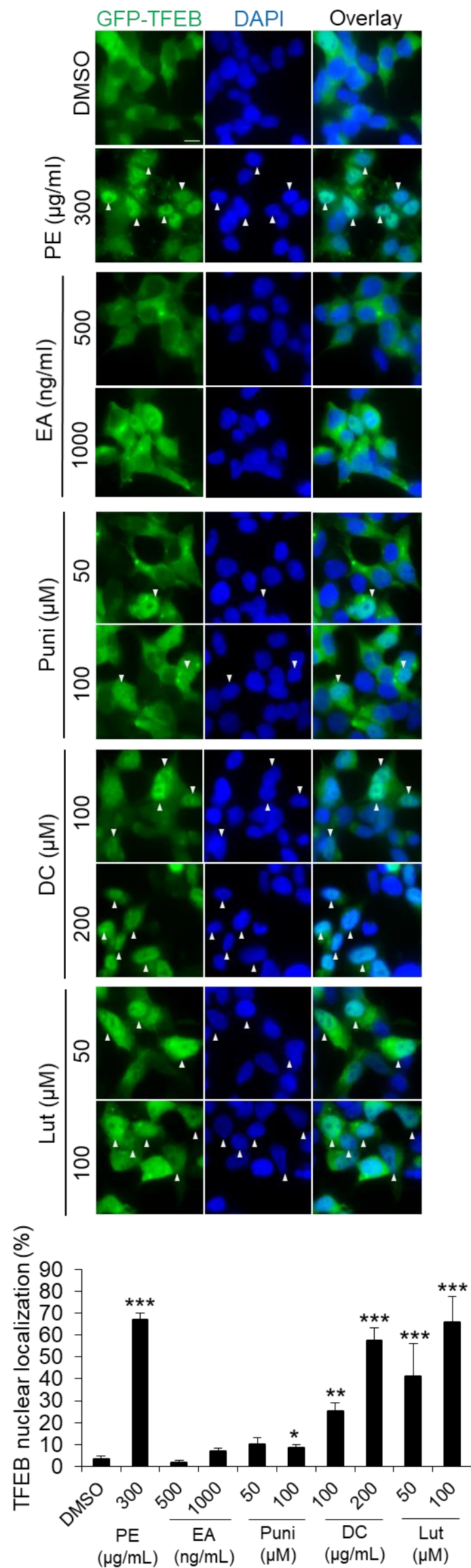
Figure 4.18 Punicalagin, delphinidin chloride and luteolin upregulate autophagosomal and lysosomal compartments via TFEB activation. Top: Immunoblots of (a) LAMP1 and (b) LC3 in SY5Y cells treated with DMSO, 300 $\mu \mathrm{g} / \mathrm{ml}$ $\mathrm{PE}$ and various concentrations of phenolic compounds as indicated for $16 \mathrm{~h}$. Bottom: Quantification of (a) LAMP1 and (b) LC3-II levels under the various treatment conditions. EA: Ellagic acid, Puni: Punicalagin, DC: delphinidin chloride and Lut: luteolin. (c) Top: TFEB localization in GFP-TFEB stable SY5Y cells treated with DMSO, $300 \mu \mathrm{g} / \mathrm{ml} \mathrm{PE}$ and various concentrations of phenolic compounds as depicted for $16 \mathrm{~h}$. Bottom: Quantification of percentage TFEB nuclear localization (white arrows) under various treatment conditions. At least 100 transfected cells per conditions were analyzed. Nuclei were stained with DAPI. (d) Top: Immunoblots of P-TFEB ${ }^{\mathrm{S} 142}$ and TTFEB in SY5Y cells treated with DMSO, $300 \mu \mathrm{g} / \mathrm{ml} \mathrm{PE}$ and various phenolic compounds (at their highest concentrations) for 16h. Right: Quantification of PTFEB $^{S 142}$ levels against T-TFEB levels after normalization against actin. All values are mean + S.E.M $(n=3-7)$. Differences against DMSO control are significant for * $p<0.05$, ${ }^{* *} p<0.01,{ }^{* * *} p<0.0005$. 


\subsubsection{Phenolic compound luteolin promotes mitophagy to protect against damaged mitochondria-induced redox toxicity}

Next, we were interested to examine if the bioactive phenolic compounds could elicit the same functional benefit as PE to alleviate mitochondrial ROS stress by facilitating mitophagy. For this analysis, we focused on Lut, which shows ability to highly upregulate autophagy-lysosomal compartments and TFEB activation (Fig 4.18).

Similar to PE, $100 \mu \mathrm{M}$ Lut enhanced the colocalization between TOMM20 and LC3 by approximately 0.4-fold as compared to the DMSO control cells under CCCP stress (Fig. 4.19a). To confirm that this enhanced colocalization is related to increased mitophagy, we examined the accumulation of mitochondria-containing autophagosomes upon further $1 \mu \mathrm{M}$ Vb treatment. Indeed, Vb resulted in a further increase in TOMM20 and LC3 colocalization by approximately 0.4-fold as compared to Lut alone under CCCP-induced stress (Fig. 4.19a). This result demonstrates that Lut promotes recruitment of the autophagosomes to the mitochondria to facilitate mitophagy under mitochondrial stress similar to PE.

Next, the ability of Lut-mediated mitophagy to protect against harmful mitochondrial ROS under CCCP stress was examined. MitoSox analysis showed that $100 \mu \mathrm{M}$ Lut effectively prevented the increase in mitochondrial ROS caused by CCCP treatment (Fig. 4.19b). However, this protection against CCCP-induced ROS was abrogated when lysosomal function was inhibited by NL (Fig. 4.19b). Thus, induction of mitophagy by Lut helps to reduce mitochondrial ROS levels by promoting degradation of damaged mitochondria. Together, our results show that Lut could account for the bioactivity of PE to upregulate mitophagy under mitochondrial stress, to protect against mitochondrial redox toxicity. 
a
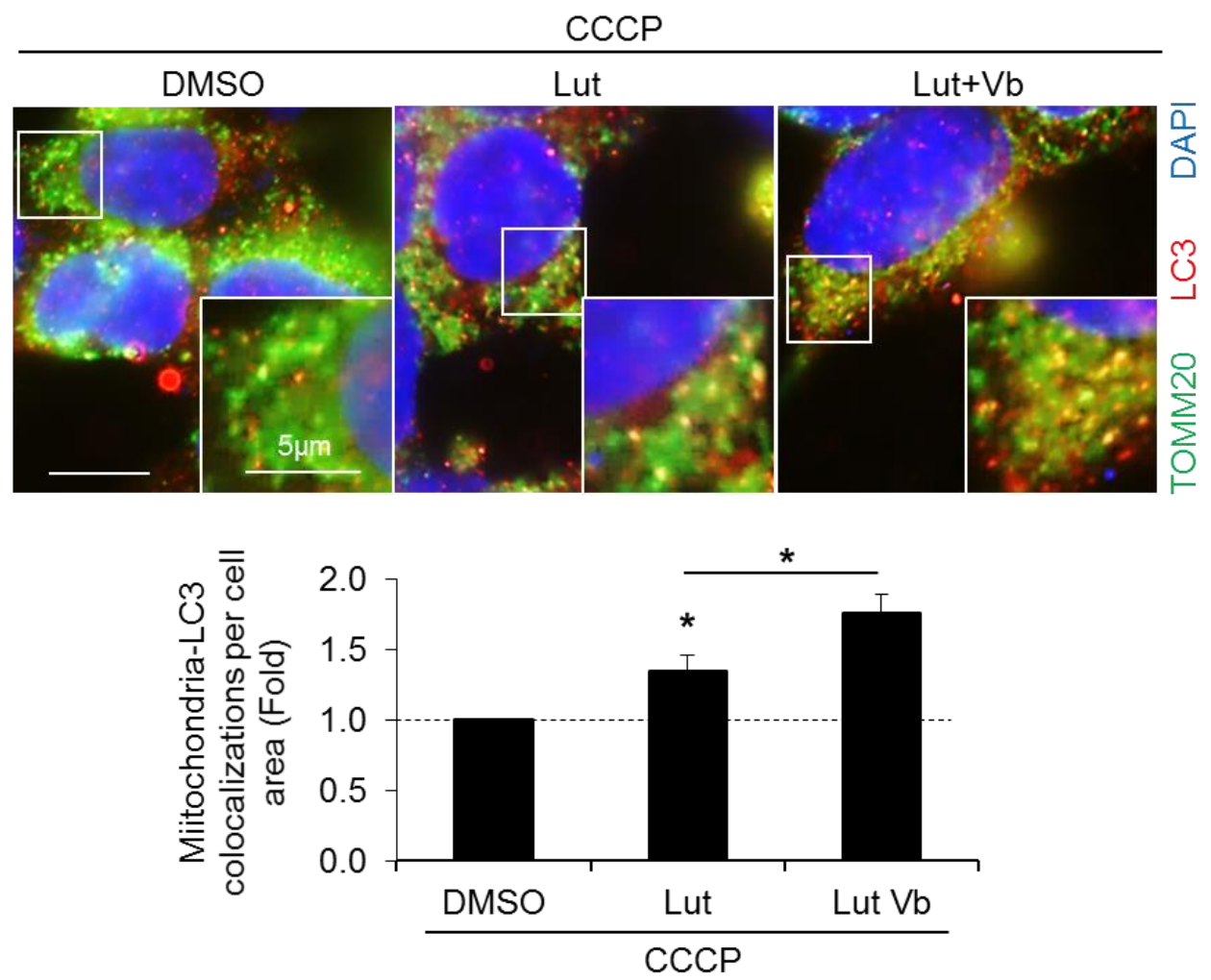

b

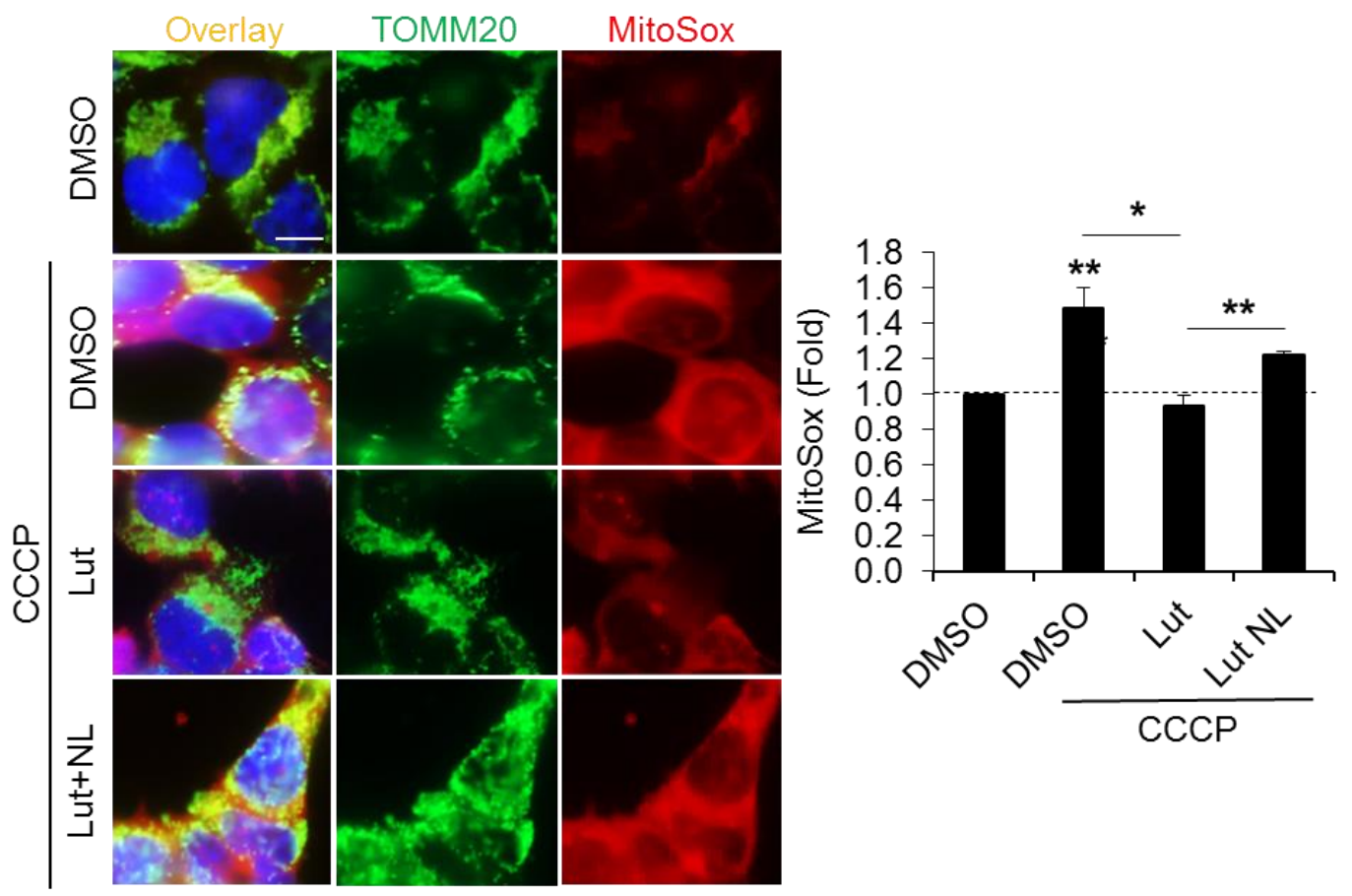


Figure 4.19 Luteolin promotes recruitment of autophagosomes to mitochondria under mitochondrial stress to facilitate mitochondria turnover and alleviate mitochondrial ROS toxicity. (a) Top: Immunofluorescence staining of TOMM20

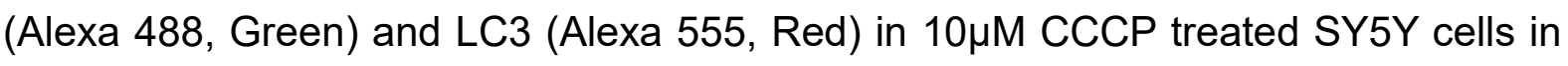
the absence or presence of $100 \mu \mathrm{M}$ Lut supplemented or not with $1 \mu \mathrm{M}$ Vb for $16 \mathrm{~h}$. Smaller inlets show close-up views of the colocalizations between TOMM20 and LC3. Bottom: Quantification of TOMM20 and LC3 colocalizations per cell area under the respective conditions. At least 30 cells per condition were analyzed. (b) Top: TOMM20 and Mitosox immunofluorescence staining in SY5Y cells treated with DMSO, 10 $\mu \mathrm{M}$ CCCP, or $10 \mu \mathrm{M} \mathrm{CCCP} \mathrm{supplemented} \mathrm{with} 100 \mu \mathrm{M}$ Lut in the absence or presence of NL for 16h. Right: Quantification of Mitosox intensity levels per cell area under various conditions. At least 30 cells per condition were analyzed. All values are mean + S.E.M $(n=3-7)$. Differences against DMSO control are significant for * $p<0.05$ and ${ }^{* *} p<0.01$. Scale bar, $10 \mu \mathrm{m}$. 


\subsection{TFEB KNOCKDOWN ABOLISHES PE'S PROTECTION AGAINST MITOCHONDRIAL MALFUNCTION}

So far, our study indicates a role of PE in protecting against CCCP-induced stress by facilitating clearance of dysfunctional mitochondria by mitophagy (Fig. 14-18). In addition, PE also upregulates autophagy-lysosomal fitness by activating TFEB (Fig. 16). With this, we hypothesized that the upregulation of TFEB signaling is the mechanistic link responsible for induction of mitophagy to negate mitochondrial stress by PE. To confirm this hypothesis, siRNA-mediated TFEB knockdown (KD) was performed in SY5Y cells to examine the consequences of TFEB ablation on PEinduced upregulation of autophagy-lysosomal compartments, as well as protection against mitochondrial malfunction under CCCP-induced stress.

\subsubsection{Ablation of TFEB function prevents PE-mediated increase in autophagy- lysosomal compartments}

The efficiency of siRNA-mediated TFEB KD in SY5Y cells was first checked by reverse transcription-PCR (RT-PCR) analysis of TFEB mRNA. Marked reduction in TFEB gene expression levels was observed upon $72 \mathrm{~h}$ transfection with TFEB siRNA reagent compared to the scrambled siRNA control (Fig. 4.20a).

Next, the effects of TFEB KD on the ability of PE to enhance the autophagy-lysosomal compartments were examined. LAMP1 and LC3 immunoblotting analyses showed that TFEB KD significantly reduced the LAMP1 (by 0.2-fold) (Fig. 4.20b) and LC3-II (by 0.2 -fold) (Fig. 4.20c) levels basally in DMSO control cells. These results are consistent with the notion that TFEB regulates the transcription of autophagic and lysosomal genes [104] and hence, inactivation of TFEB will reduce the autophagylysosomal compartments. With PE treatment, scrambled control cells showed the expected increase in LAMP1 and LC3-II levels. However, such PE-induced upregulation of LAMP1 and LC3-II levels was not observed in TFEB KD cells (Fig. $4.20 \mathrm{~b}$ and $\mathrm{c}$ ). Our results show that the loss of TFEB function perturbs the upregulation of autophagy-lysosomal compartments by PE. Thus, PE enhancement of autophagy activity is dependent on TFEB activation. 
a

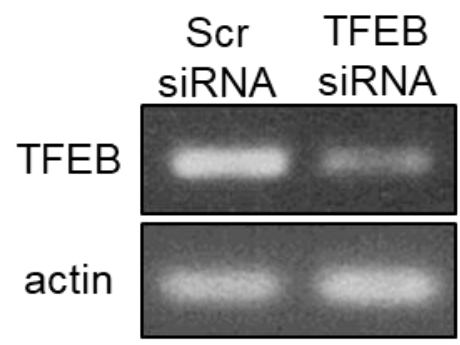

b

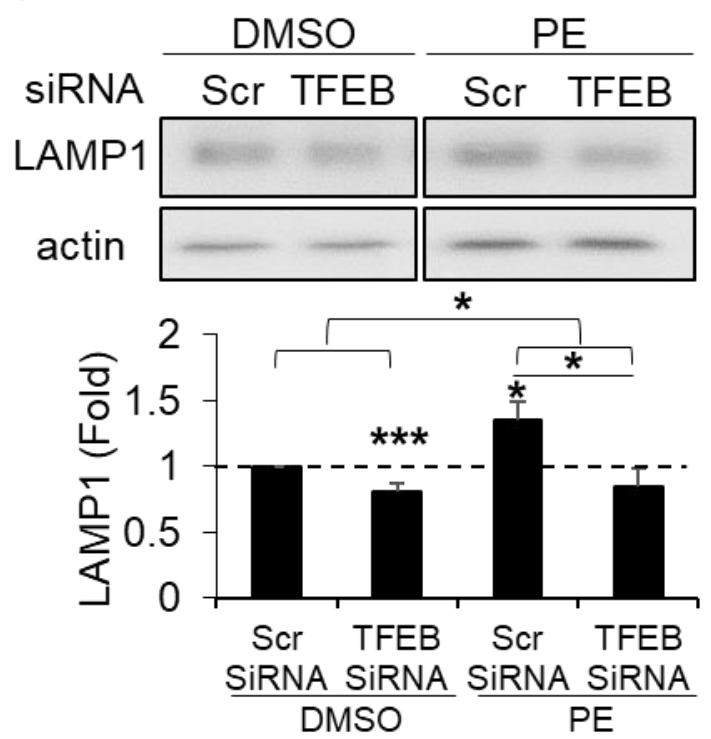

C
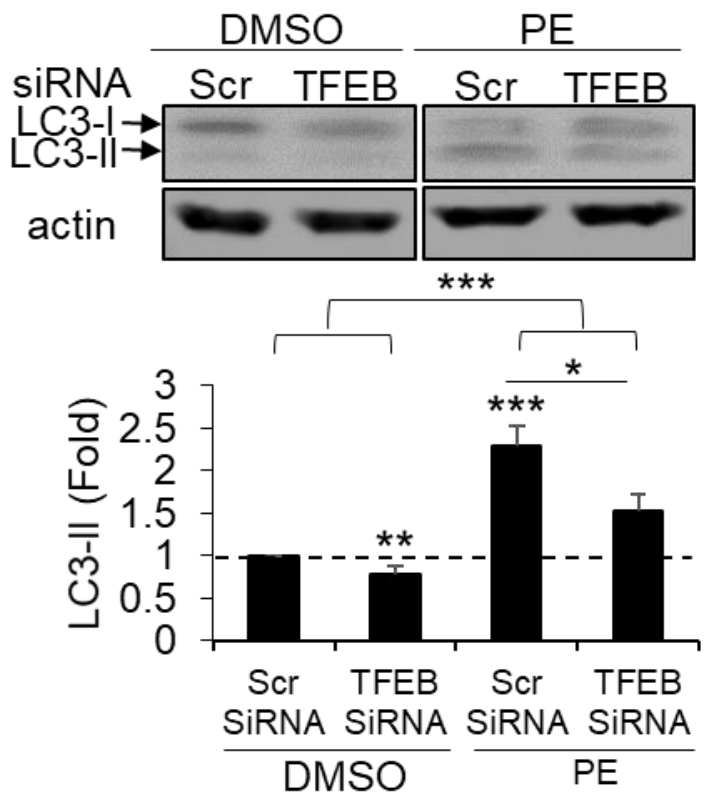
Figure 4.20 siRNA-mediated TFEB knockdown (KD) abolishes PE-induced upregulation of autophagosomal and lysosomal compartments. (a) RT-PCR analysis of TFEB expression levels in SY5Y cells after $72 \mathrm{~h}$ transfection with $25 \mu \mathrm{M}$ scrambled (Scr) siRNA or TFEB siRNA. Top: Immunoblots of (b) LAMP1 and (c) LC3 in scrambled control and TFEB KD cells treated with DMSO or $300 \mu \mathrm{g} / \mathrm{ml} \mathrm{PE}$ for $16 \mathrm{~h}$. Bottom: Quantification of (b) LAMP1 and (c) LC3-II levels in the corresponding cells, expressed as fold change against scrambled siRNA control cells treated with DMSO. All values are mean + S.E.M ( $n=3-7)$. Differences are significant for * $p<0.05$, ** $p<0.01,{ }^{* * *} p<0.0005$. 


\subsubsection{Ablation of TFEB function reduces PE-mediated mitophagy and protection against mitochondrial ROS redox toxicity}

Next, the consequences of TFEB KD on PE-induced mitophagy and protection against mitochondrial stress in SY5Y cells were investigated. As before, $300 \mu \mathrm{g} / \mathrm{ml}$ PE significantly increased the TOMM20 and LC3 colocalization basally and under CCCP stress by approximately 1 and 2 -folds respectively in the scrambled control cells (Fig. 4.21a). Interestingly, TFEB KD perturbed the effects of PE on enhancing mitochondria and autophagosome colocalization under CCCP stress, but not under basal condition (Fig. 4.21a). This result shows that under physiological condition, PE encourages the recruitment of autophagosomes to the mitochondria in a TFEB-independent manner.

To further confirm that TFEB signaling is instrumental in PE-induced mitophagy during CCCP stress, TOMM20 flux was analyzed in the scrambled control and TFEB KD cells co-treated with $10 \mu \mathrm{M}$ CCCP and $300 \mu \mathrm{g} / \mathrm{ml}$ PE (Fig. 4.21b). Loss of TFEB function significantly reduced the TOMM20 flux during CCCP stress by approximately $50 \%$ as compared to scrambled control cells (Fig. 4.21b). Thus, loss of TFEB signaling abrogates the ability of PE to enhance mitophagy under mitochondrial stress.

Next, we investigated if this loss of mitophagy caused by TFEB ablation reduces PE protection against mitochondrial ROS during CCCP insult. While clear reduction in MitoSox signal was observed in CCCP and PE co-treated scrambled control cells, the reduction in the similarly treated TFEB KD cells was partially prevented (Fig. 4.21c). Collectively, these results highlight that ablation of TFEB prevents PE-induced mitophagy under mitochondrial stress, by reducing the recruitment of the autophagosomes to the mitochondria. Likely, the perturbation of PE-induced mitophagy reduces the protection by PE against mitochondrial ROS-induced toxicity. 

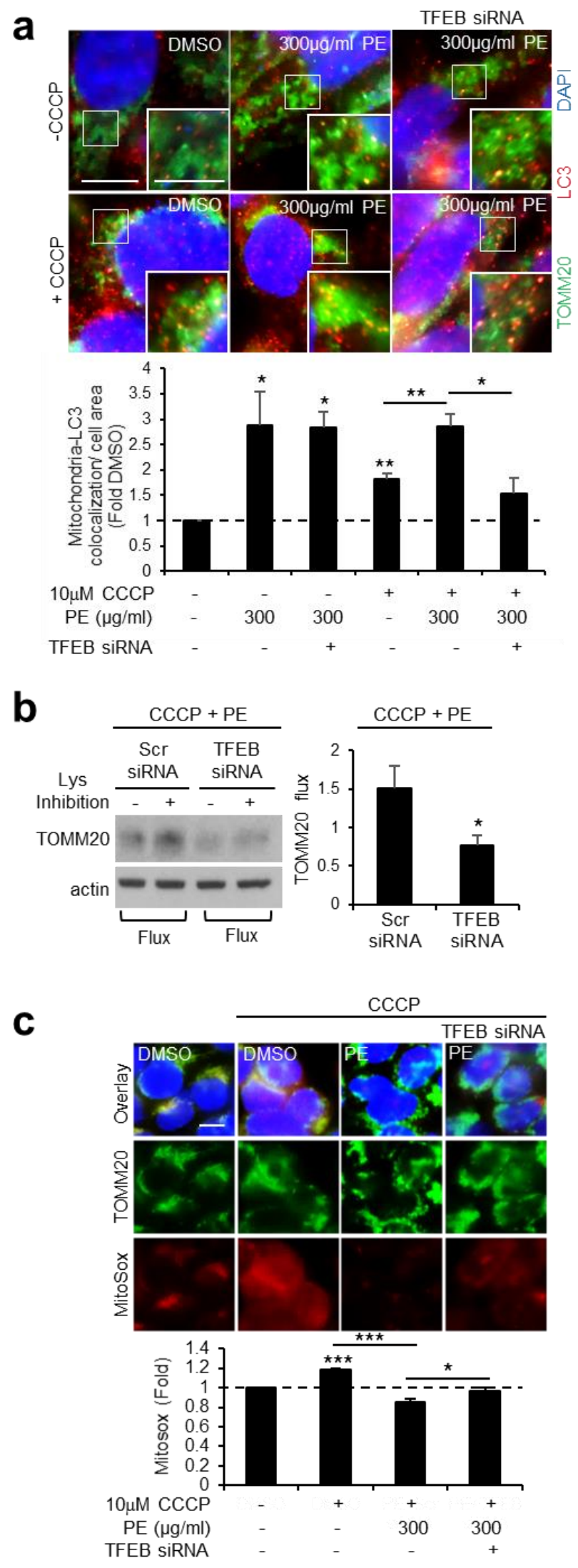
Figure 4.21 TFEB KD perturbs recruitment of autophagosomes to mitochondria, reduces PE-induced mitophagy and PE protection against mitochondrial ROS toxicity. (a) Top: Immunofluorescence staining of TOMM20 (Alexa 488, green) and LC3 (Alexa 555, red) in scrambled control and TFEB KD cells untreated or treated with $10 \mu \mathrm{M}$ CCCP supplemented with either DMSO or $300 \mu \mathrm{g} / \mathrm{ml}$ PE for $16 \mathrm{~h}$. Bottom: Quantification of TOMM20-LC3 colocalization per cell area per treatment condition. (b) Left: Immunoblot of TOMM20 in CHX treated scrambled control and TFEB KD cells subjected to $10 \mu \mathrm{M} \mathrm{CCCP}$ and $300 \mu \mathrm{g} / \mathrm{ml} \mathrm{PE}$ in the absence or presence of lysosomal inhibition. Right: Quantification of TOMM20 flux in scrambled control and TFEB KD cells under the stated conditions. (c) Top: Immunofluorescence images of TOMM20 (Alexa 488, green) and MitoSox (Red) in scrambled control and TFEB KD cells under the various treatment conditions (16h) indicated. Bottom: Quantification of MitoSox intensity per cell area per treatment condition. At least 20 cells from random fields were analyzed per experimental set. Nuclei were stained with DAPI. All values are mean + S.E.M $(n=3-7)$. Differences are significant for ${ }^{*} p<0.05,{ }^{* *} p<0.01,{ }^{* * *} p<0.0005$. Scale bar is $10 \mu \mathrm{m}$ unless otherwise stated. 


\section{CHAPTER 5}

DISCUSSIONS, FUTURE

DIRECTIONS AND CONCLUSION 


\subsection{OVERVIEW}

Quality control constitutes a central part of cellular physiology [1]. Autophagy, particularly selective autophagy, is a quality control pathway that identifies, isolates and targets unwanted intracellular components for lysosomal degradation [441]. The selective degradation of protein aggregates (aggrephagy) and mitochondria (mitophagy) are the most well-studied types of selective autophagy. Impairment of both pathways has been heavily implicated in neurodegeneration [32, 283]. Hence, aggrephagy and mitophagy are attractive targets for therapies against neurodegenerative diseases.

In our present study, the molecular determinants as well as the biomolecules that can regulate aggrephagy and mitophagy have been characterized. In the first part, we identified a novel function of phosphorylation in regulating different types of Synphilin-1 (Sph1)-mediated aggrephagy, where the interplay between phosphorylation and ubiquitination is important for inducible aggrephagy under cellular stress. In the next part, we identified polyphenols-enriched pomegranate extract (PE) as an inducer for selective clearance of damaged mitochondria under mitochondrial stress, and delineated the mechanism that underlies PE-induced mitophagy. 


\subsection{INTERPLAY BETWEEN PHOSPHORYLATION AND UBIQUITINATION MODULATES SYNPHILIN-1 AGGREPHAGY BEHAVIOR}

Sph1-mediated aggrephagy represents a non-canonical way of selectively degrading the small cytosolic aggregates (Agg) basally (basal aggrephagy), and the large perinuclear aggresome (Agm) under cellular stress (inducible aggrephagy) [18]. The former clearance process is independent on K63-linked polyubiquitination (K63U); whereas, K63U on Sph1 is important for inducible aggrephagy by promoting the recruitment of class III PI3-kinase onto the Agm surface to initiate autophagosome biogenesis [18].

The present study uncovered a role of phosphorylation mediated by the kinases PKC, CKII and GSK3 $\beta$ in regulating the autophagic susceptibility of Sph1 Agg and Agm towards basal and inducible aggrephagy respectively. Further, Sph1 phosphorylation increases under proteasomal stress condition, which switches the behavior of Sph1 from mediating Agg basal aggrephagy to Agm inducible aggrephagy. Additionally, this study unravels an interplay between phosphorylation and ubiquitination in regulating selective clearance of Agm by inducible autophagy. Thus, altering the phosphorylation levels on Sph1 in response to cellular conditions represents an elegant way that allows the cells to toggle between constitutive removal of small Agg basally, to high capacity removal of Agm upon stress conditions.

\subsubsection{Phosphorylation represents a novel molecular determinant to regulate selective clearance of Agg by basal autophagy}

In-silico (Fig. 3.2) and mass spectrometry (Fig. 3.3) analyses identified additional phosphorylation sites on Sph1 previously not reported. The readout from the PhosTag SDS-PAGE showed that PKC, CKII and GSK3 $\beta$ isoforms phosphorylate Sph1 (Fig. 3.6). CKII and GSK3 $\beta$ have been reported to phosphorylate Sph1 to influence Sph1 aggregation propensity [201, 202]. However, the action of PKC isoforms on Sph1 has not been reported. The PKC family consists of fifteen isozymes in humans [442]. Of these, 
PKCa is the major isoform and PKCy is a neuronal-specific isoform. Our study identified PKCa and PKCY as novel kinases that phosphorylate Sph1.

The co-immunoprecipitation (co-IP) assay identified PKCa, PKCY and GSK3 $\beta$ as interactors of Sph1 (Fig. 3.4a, b and d). Additionally, these kinases also localized to the Agg formed by Sph1 (Fig. 3.5d). On the other hand, the interaction between CKII $\beta$ and Sph1 was not detected in the co-IP assay (Fig. 3.4c), but CKIIß localized to the Sph1 Agg (Fig. 3.5d). CKII and GSK3 $\beta$ have been reported to interact with Sph1 [201, 202]. Our observed interaction between Sph1 and GSK3 $\beta$ aligned with the reported data. However, CKII $\beta$ did not interact with Sph1 in our study, a contrary to the reported finding. The presence of CKII $\beta$ isoform in the Agg formed by Sph1 however, suggests that CKII $\beta$ may only be sequestered into the Agg inclusions after they are formed. Hence, the co-IP assay did not detect any interactions between the soluble forms of CKII $\beta$ and Sph1 in our study. The presence of PKC $\alpha$ and PKC $\gamma$, along with CKII $\beta$ and GSK3 $\beta$ isoforms in the Agg formed by Sph1 provided an insight for the potential role of these kinases in influencing Sph1 basal aggrephagy behavior.

Chemical inhibition of PKC, CKII and GSK3 $\beta$ isoforms perturbed the basal clearance of Sph1 Agg (Fig. 3.9). Although PKC, CKII and GSK3 $\beta$ are implicated in the regulation of autophagy [370, 371], inhibition of these kinases did not affect the general cellular autophagy flux in our study (Fig. 3.13). This finding demonstrates the effects observed from the kinase inhibitions are specific to Sph1-mediated basal aggrephagy. Similar to the chemical inhibition assay, isoform-specific genetic knockdown (KD) of PKCa, PKCY and GSK3 $\beta$ perturbed the Agg turnover basally (Fig. 3.10a, b and d). However, CKII K KD did not affect basal aggrephagy (Fig. 3.10c), suggesting that the $\beta$ isoform of the CKII complex is not responsible for Agg turnover by basal aggrephagy.

Originally thought to be a constitutive process that promotes non-selective turnover of proteins for homeostasis [123, 335], several reports now suggest some forms of substrate selectivity for basal autophagy [18, 122, 443, 444]. However, the precise mechanisms underlying substrate selectivity by basal autophagy remains inadequately understood. 
Our present study identified phosphorylation mediated by PKC $\alpha$, PKCY and GSK3 $\beta$ as a critical determinant for mediating selective autophagic clearance of Sph1 Agg under basal condition. PKC isoforms have not been reported to interact or modulate the behavior of Sph1. Hence, our study uncovered PKCa and PKCY as new interacting partners of Sph1 to regulate basal aggrephagy. Although GSK3 $\beta$ has been reported to interact and phosphorylate Sph1 [202], our study uncovered an additional role for GSK3 $\beta$-mediated phosphorylation for Sph1 basal aggrephagy. CKII has been reported to phosphorylate Sph1, and the $\beta$ isoform is critical for interaction between CKII complex and Sph1 [201]. Our findings on the localization of CKII $\beta$ to the Agg without affecting the turnover basally suggest CKII $\beta$ as an unlikely molecular player for Sph1 basal aggrephagy. CKII is a heterotetrameric kinase consisting of two catalytic ( $\alpha$ or $\alpha 0)$ and two regulatory $\beta$ subunits [445]. Further studies are needed to explore the possibility of the catalytic CKIla isoform as a determinant in regulation of Sph1 basal aggrephagy.

\subsubsection{Phosphorylation regulates selective clearance of Agm by inducible aggrephagy}

$\mathrm{K} 63 \mathrm{U}$ is an important determinant for clearance of Agm formed by Sph1 under cellular stress [18]. In the present study, phosphorylation is also identified as a determinant critical for modulating inducible aggrephagy. Surprisingly, all the four kinase isoforms, PKCa, PKC $\gamma, C K I I \beta$ and GSK3 $\beta$, are important for inducible aggrephagy.

PKC $\alpha$, PKC $\gamma, C K I I \beta$ and GSK3 $\beta$ localized to the Agm formed by Sph1 (Fig. 3.5c). CKII $\beta$ localized as a "halo-ring" structure enclosing the Agm (Fig. 3.5c), in parallel with the CKII localization pattern in the Lewy body (LB) of PD patients [446]. Similarly, PKCa, PKCY and GSK3 $\beta$ either localize as a "halo-ring", or completely colocalized with the Agm formed by Sph1 (Fig. 3.5c). Chemical inhibition of PKC, CKII and GSK3 $\beta$ perturbed clearance of Agm by starvation-induced aggrephagy (Fig. 3.11), and isoform-specific KD of PKCa, PKCY, CKII $\beta$ and GSK3 $\beta$ isoforms inhibited Agm clearance (Fig. 3.12). These observations demonstrated that PKCa, PKCy and GSK3 $\beta$ are important for both basal and inducible aggrephagy, but the CKII $\beta$ isoform is more critical for the latter process. 
The identification of phosphorylation as a critical determinant for clearance of Agm on top of the known K63U function indicates a possible interplay between phosphorylation and ubiquitination in regulating inducible aggrephagy of Sph1.

\subsubsection{GSK3 $\beta$ mediates the crosstalk between phosphorylation and K63U to regulate selective clearance of Agm by inducible aggrephagy}

Crosstalk between phosphorylation and ubiquitination has been shown to regulate selective autophagy [10]. In the present study, chemical inhibition of GSK3 $\beta$ reduced the levels of K63U linkage on Sph1 under proteasomal stress (Fig. 3.14), highlighting a potential interplay between phosphorylation and ubiquitination in Sph1-mediated inducible aggrephagy. Parkin is an E3 ligase known to interact with Sph1, and mediate K63U on Sph1 to promote Agm formation and clearance under proteasomal stress [198, 199]. GSK3 $\beta$ interacts with Parkin under proteasomal stress (Fig. 3.15d), and chemical inhibition of GSK3 $\beta$ perturbed the interaction between Sph1 and Parkin under proteasomal inhibition (Fig. 3.16). Coupled with finding that GSK3 $\beta$ also interacts with Sph1 under proteasomal stress (Fig. 3.4d), our study suggests a possible tripartite interaction between GSK3 $\beta$, Sph1 and Parkin under proteasomal dysfunction. Furthermore, GSK3 $\beta$ inhibition also reduced the levels of phosphorylated Parkin ${ }^{565}$ and the cellular pool of phosphorylated ubiquitin ${ }^{565}$ under proteasomal stress (Fig. 3.17), the two critical determinants for activation of Parkin [251-253, 395]. Collectively, the findings in our present study identifies GSK3 $\beta$ as a novel regulator for Parkin activation and the association between Parkin and Sph1 under proteasomal stress. This regulatory mechanism might underlie the enhancement of K63U on Sph1 for inducible aggrephagy under proteasomal dysfunction. 
We propose a molecular mechanism where the crosstalk between phosphorylation and ubiquitination, mediated by the interplay between GSK3 $\beta$ and Parkin, potentiates the selective clearance of Sph1 Agm by inducible aggrephagy during proteotoxicity induced by proteasomal impairment (Fig. 5.1). Upon onset of proteasomal stress, Parkin is recruited to the Sph1-GSK3 $\beta$ complex. GSK3 $\beta$ mediates the activation of Parkin by phosphorylating the $\mathrm{E} 3$ ligase and ubiquitin at $\mathrm{S} 65$, and enhances the interaction between Sph1 and Parkin. Hence, Parkin can efficiently facilitate the addition of K63U linkages onto Sph1 to promote clearance of Agm by inducible aggrephagy.

Activation of Parkin by the kinase PINK1 is important to mediate selective clearance of damaged mitochondria under mitochondrial stress (mitophagy) [251-253, 395]. The identification of GSK3 $\beta$ as a potential regulator of Parkin activation during proteotoxicity suggests Parkin may partner with different kinases in response to different cellular stressors to mediate either mitophagy or aggrephagy. Indeed, the activity of Parkin has been shown to be influenced by its binding partners. For example, the type of E2 ubiquitinconjugating enzymes specifies the activity of Parkin in the ubiquitination cascade to modulate different processes. Parkin partners with E2 enzymes UbcH5 ubiquitinconjugating enzyme (UBE2D) and ubiquitin conjugating enzyme E2 L3 (UBE2L3) to modulate its own activation and translocation to the mitochondria for mitophagy [447]. On the other hand, Parkin partners with E2 enzyme Ubc13 to mediate K63U on protein substrates for aggrephagy [200, 448, 449]. Hence, in regard to selective autophagy, proteasomal stress may induce the association between GSK3 $\beta$ and Parkin to mediate aggrephagy, while onset of mitochondrial stress facilitates a switch in Parkin binding partner to PINK1 to mediate mitophagy. Further investigations are necessary to determine if changes in cellular stress underlie the molecular switching of interaction partners on Parkin to favor one type of selective autophagy over another. 


\subsubsection{PKC and CKII modulates the selective clearance of Agm by inducible aggrephagy}

Unlike GSK3 $\beta$, the mechanisms underlying the regulation of inducible aggrephagy by PKC and CKII are less defined in the present study. Although PKCa interacts with Parkin under proteasomal stress (Fig. 3.15a), chemical inhibition of PKC neither altered the K63U linkages on Sph1 (Fig. 3.14b) nor the activation of Parkin (Fig. 3.17). Similarly, chemical inhibition of CKII did not alter both events (Fig. 3.14c and 3.17). Hence, the regulation of inducible aggrephagy by PKC and CKII is distinct from GSK3 3 . The list of plausible mechanisms to which PKC and CKII can adopt to modulate the inducible aggrephagy behavior of Sph1 may be innumerable; however, the observation that PKC and CKII did not modulate K63U on Sph1 suggests both kinases may affect inducible aggrephagy by influencing the K63U-mediated downstream events.

$\mathrm{K} 63 \mathrm{U}$ on the Agm is important for stabilizing the protein structure to promote recruitment of the class III PI3-kinase complex to initiate autophagosome biogenesis [18]. Rather than altering the levels of K63U on Sph1 to influence inducible aggrephagy, PKC and CKII may influence the recruitment of the components from the class III PI3-kinase complex to the Agm. Examination of the association between Sph1 Agm with the components of the class III PI3-kinase complex like the zinc finger FYVE-type containing 1 (DFCP1) and vacuolar sorting protein 34 (Vps34) under PKC and CKII inhibitions may help to decipher if both groups of kinase affect inducible aggrephagy via recruitment of the autophagic machinery to the Agm.

On a side note, chemical inhibition of CKII perturbed the interaction between Sph1 and Parkin (Fig. 3.16). However, at this present stage of the study, we can only conclude that CKII is important for the interaction between Sph1 and Parkin. The mechanism of how CKII-mediated enhanced association consequentially mediate inducible aggrephagy is not understood. Unlike GSK3 $\beta$, the enhanced interaction between Sph1 and Parkin mediated by CKII is not crucial for facilitating K63U linkages on Sph1 for inducible 
aggrephagy. Hence, CKII-mediated interaction between Sph1 and Parkin might modulate Sph1 in other ways to influence inducible aggrephagy.

\subsubsection{Proteasomal stress potentiates the switch from basal to inducible aggrephagy}

Autophagy occurs at a basal level in all cells and is upregulated in response to diverse stressors, including starvation, oxidative stress and pathogen invasions [450]. The ability of the cell to only mediate constitutive clearance of the Agg basally, and clearance of the Agm upon cellular stress, suggests that a molecular switch exists to dictate the choice of the clearance pathway under resting and stress states. The consistent observation of an enhanced phosphorylation-associated phenomenon upon proteasomal stress, including phosphorylation on Sph1 (Fig. 3.6b) and the interaction of PKC $\alpha$ and GSK $\beta$ with Sph1 (Fig. 3.4a and $\mathrm{d}$ ), suggest that proteasomal dysfunction triggers an enhanced phosphorylation on Sph1 to potentiate the switch from basal Agg clearance to inducible Agm clearance. We propose a mechanism where phosphorylation is important for facilitating constitutive clearance of Sph1 Agg under basal condition; however, onset of proteasomal stress may enhance the interaction between the kinases and Sph1 and phosphorylation on Sph1 (Fig. 5.1). For the case of PKCa and GSK3 $\beta$, the enhanced interaction with Sph1 under proteasomal stress further engages Parkin to Sph1 (Fig. $3.15 a$ and d). Specifically, for GSK3 $\beta$, the recruitment of Parkin is followed by activation of the E3 ligase and an enhanced interaction between Parkin and Sph1 to potentiate K63U on Sph1 (Fig. 5.1).

In fact, modulation of the levels of post-translational modification has been reported to specify Sph1 degradation fate. For example, subtle changes in the relative proportions of the $\mathrm{K} 48 \mathrm{U}$ and $\mathrm{K} 63 \mathrm{U}$ ubiquitin linkages on Sph1 modify the Agm autophagic susceptibility [18]. Here, we also reported a similar phenomenon, where enhanced phosphorylation on Sph1 upon proteasomal stress potentially allows the cell to switch from basal Agg removal to favor disposal of the Agm by the inducible aggrephagy pathway. Moreover, regulations of the basal and inducible aggrephagy are mediated by the same group of kinases, PKC, 
CKII and GSK $\beta$. The could allow the cells to rapidly respond to proteasomal stress via subtle changes in the phosphorylation status, instead of having to recruit additional components to modulate the switch. However, the key question to whether PKC, CKII and GSK3 $\beta$ act cooperatively or independently to modulate Sph1 aggrephagy behavior remained to be investigated. Further investigation is also necessary to validate if an enhanced phosphorylation during proteasomal inhibition mediates the switch from basal to inducible aggrephagy. For example, a comparison of the phosphorylation sites on Sph1 identified by mass spectrometry under basal and proteasomal stress conditions may provide an insight to the potential sites on Sph1 that are relevant under proteasomal inhibition. Subsequently, these potential sites could be mutated into the phosphoinactive amino acid like alanine to examine if the mutations abolish Sph1-mediated inducible aggrephagy upon onset of proteasomal stress.

\subsubsection{Sph1 in health and diseases: Sph1 as a novel adaptor protein to promote clearance of protein inclusions}

The significance of cargo adaptor proteins in neurodegenerative diseases has been increasingly recognized, due to the identification of genetic mutations in the cargo adaptor proteins as a common mechanism for disease pathogenesis [12]. While gene replacement therapy offers a viable treatment option for genetic disorders, the current lack of knowledge and understanding of the treatment compromises the safety of the technique for clinical use [303].

Sph1-mediated basal and inducible aggrephagy processes are independent of cargo adaptor proteins [18]. In p62 and NBR1 KO cells, Sph1 continues to promote turnover of the Agg basally and the Agm upon starvation-induced stress [18]. Moreover, Sph1 not only promotes the removal of inclusions formed by its own protein species, but also facilitates the removal of several PD-linked proteins that are not amenable to autophagic clearance [181]. This dispensability of cargo adaptors in Sph1-mediated aggrephagy, and the ability of Sph1 to also promote selective clearance of other PD-linked pro-aggregating proteins suggest that Sph1 may compensate for the loss of cargo adaptor proteins activity 
in familial or hereditary forms of PD to facilitate clearance of protein inclusions. However, whether Sph1 is able to mediate clearance of all types of protein inclusions in other neurodegenerative conditions remains questionable. So far, only inclusions formed by coaggregating proteins from the PD-linked LB with Sph1 are amenable for autophagic clearance [181]. Nonetheless, delineating the intricate mechanisms to how Sph1 can mediate both basal and stress-induced autophagy in the present study, provided an understanding on how Sph1 activity as an adaptor protein may be fine-tuned by posttranslational modifications to mediate clearance of the different types of inclusions. 


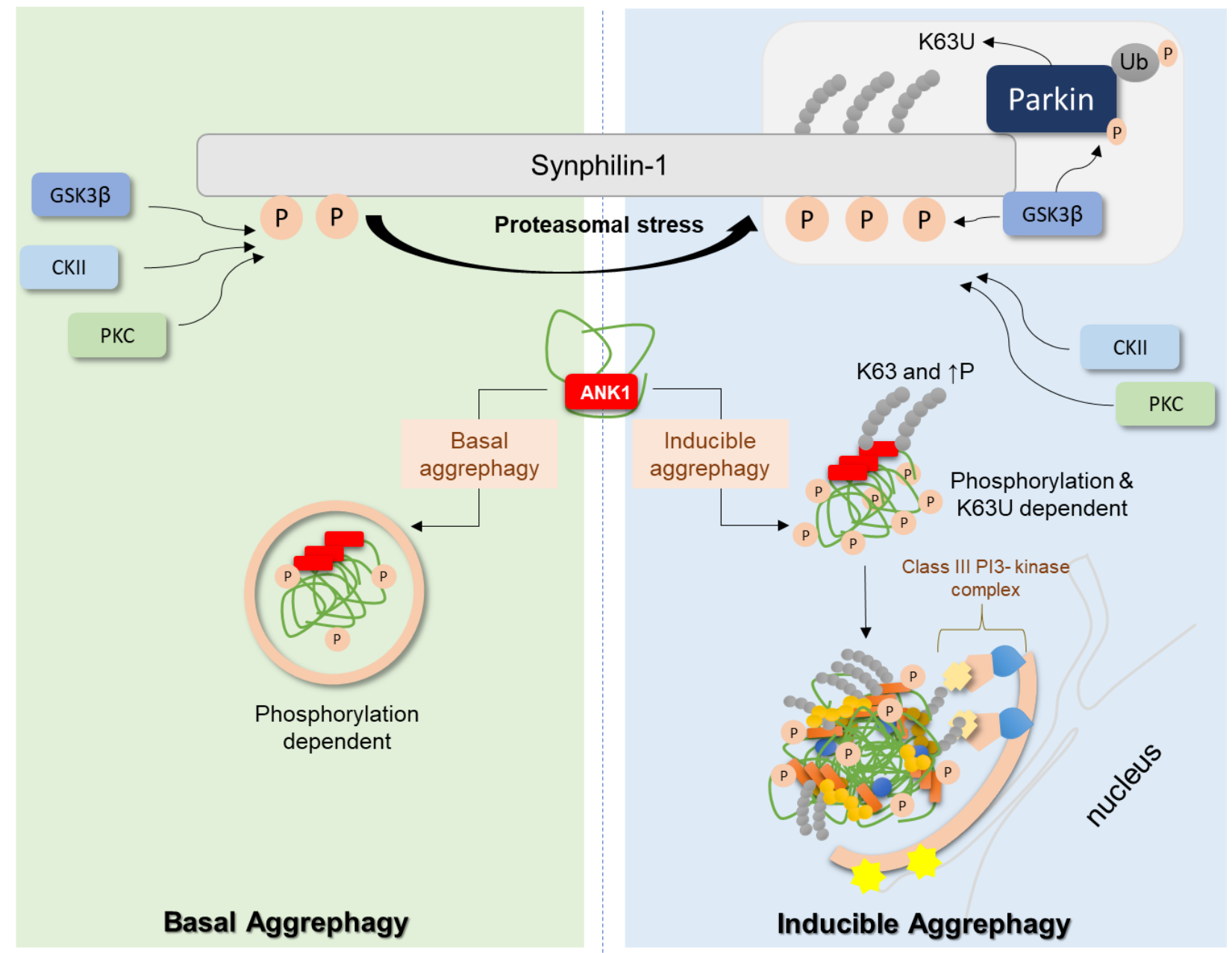

Figure 5.1 Phosphorylation regulates Sph1-mediated aggrephagy behavior. Schematic depicting enhanced phosphorylation of Sph1 upon proteasomal stress to serve as the molecular signal to switch from basal to inducible aggrephagy. The interplay between phosphorylation and ubiquitination in regulating Sph1 inducible aggrephagy behavior is also shown. PKC, CKII and GSK3 $\beta$ phosphorylate Sph1 and promote the turnover of Sph1 Agg by basal aggrephagy. Increased binding occurs between Sph1 and PKC $\alpha$ as well as GSK3 $\beta$ under proteasomal stress to enhance Sph1 phosphorylation to mediate the switch from basal to inducible aggrephagy. For GSK3ß-mediated inducible aggrephagy, GSK3 $\beta$ not only promotes Sph1 interaction with Parkin, but also activates Parkin E3 ligase activity by phosphorylation and increasing phospho-ubiquitin levels. These events enhance the K63U levels on Sph1 under proteasomal stress, and promote 
the recruitment of class III PI3-kinase complex to the Agm to mediate inducible aggrephagy. While PKC and CKII isoforms are also important for inducible aggrephagy, the mechanism underlying the clearance of Agm by both kinases remains unclear. 


\subsection{POMEGRANATE PROMOTES MITOPHAGY VIA TFEB ACTIVATION TO MAINTAIN MITOCHONDRIAL HEALTH AND FITNESS}

Modulation of lifestyle to include dietary intake of polyphenols-enriched food like pomegranate has gained popularity as a therapy against neurodegeneration and aging [372, 373, 402]. Emerging evidence shows that the beneficial effects of pomegranate and its constituents are partly linked to autophagy modulation [16, 410, 435, 451]. Our study uncovered a role of pomegranate extract $(\mathrm{PE})$ and its associated polyphenols in regulating selective clearance of damaged mitochondria (mitophagy) for mitochondrial health and fitness under stress condition. Mechanistically, PE upregulates the autophagosomal and lysosomal compartments via TFEB activation to mediate mitochondria clearance. These findings suggest that intake of pomegranate is a promising avenue to alleviate neurodegenerative conditions associated with mitochondrial dysfunction.

\subsubsection{PE upregulates autophagy}

Pomegranate and its constituents have been shown to induce autophagy in different cells lines, including glioblastoma, syncytiotrophoblast, papillary thyroid and hepatocellular carcinoma cells [410, 411, 435, 452]. Here in our study, we found that PE upregulates the levels of autophagosome and lysosome, as well as the autophagy flux in neuronal SY5Y cells (Fig 4.1-4.4). This finding re-emphasizes the universal nature of $P E$ to induce autophagy in various cell types. The ability of PE to upregulate autophagy in the postmitotic of neuronal cells is particularly important, as the neuronal cells rely heavily on autophagy to degrade unwanted and damaged intracellular components to preserve cellular integrity [263]. 


\subsubsection{PE activates TFEB via lysosomal-Ca ${ }^{2+}$ signaling independent of $\mathrm{mTOR}$}

TFEB is a transcription factor for autophagosomal and lysosomal genes to upregulate autophagy under starvation [104]. Our study uncovered the activation of TFEB by PE as the molecular mechanism underlying PE-induced autophagy in neuronal SY5Y cells. PE reduces the inhibitory S142 phosphorylation on TFEB (Fig. 4.6), and induces TFEB translocation to the nucleus to potentiate transcription of autophagy-related genes (Fig. 4.5).

mTORC1 and ERK1/2 are kinases reported to phosphorylate TFEB at $\mathrm{S} 142$ to inhibit TFEB nuclear localization and action [91, 118, 119]. While PE has no effects on ERK1/2 signaling (Fig. 4.7), PE upregulates AKT-mTORC1 signaling (Fig. 4.8) that supposedly inhibit autophagy by preventing TFEB nuclear translocation. The lysosomal- $\mathrm{Ca}^{2+}$ signaling pathway represents another mechanism to induce TFEB nuclear shuffling independent of mTORC1 activity. In this pathway, the release of lysosomal $\mathrm{Ca}^{2+}$ into the cytosol activates the phosphatase calcineurin [120]. Activated calcineurin then dephosphorylates TFEB at S142 to induce TFEB nuclear localization [120]. In our present study, inhibition of calcineurin activity and addition of $\mathrm{Ca}^{2+}$ chelator perturbed $\mathrm{PE}$-induced TFEB nuclear localization (Fig. 4.9), highlighting the importance of $\mathrm{Ca}^{2+}$ and calcineurin activity in the process. Hence, PE potentially induces $\mathrm{Ca}^{2+}$ release from the lysosomes to trigger calcineurin-dependent dephosphorylation of cytosolic TFEB at S142 to facilitate TFEB nuclear translocation. This event may override the $\mathrm{mTORC1}$ signaling to induce TFEB nuclear localization. Further investigation on the effect of PE-induced TFEB nuclear localization in calcineurin KD model is important to determine if the TFEB shuffling is dependent on the lysosomal- $\mathrm{Ca}^{2+}$ signaling pathway. In addition, monitoring the changes in the lysosomal $\mathrm{Ca}^{2+}$ level under the influence of $\mathrm{PE}$ can also provide another supporting evidence for the role of $\mathrm{Ca}^{2+}$ in modulating PE-induced TFEB nuclear localization and activation. 


\subsubsection{PE-induced autophagy does not contribute to cellular proteostasis}

Autophagy represents an important pathway for clearance of damaged and unwanted proteins to preserve cellular proteostasis [11]. Here in our study, PE protected against the accumulation of oxidized proteins (Fig.10) and protein aggregates (Fig. 4.11). However, autophagy inhibition did not abrogate these protective effects of PE (Fig. 4.10 and 4.11), thus demonstrating PE-induced autophagy was neither involve in attenuating oxidative stress via autophagic clearance of oxidized proteins, nor protect against protein aggregates-induced toxicity by enhancing aggrephagy. For the latter, PE seems to reduce the formation of protein aggregates rather than enhancing the clearance.

Pomegranate and its associated polyphenols have been widely reported to alleviate oxidative stress via its antioxidant activity [372, 373, 402]. Hence, the antioxidant property of PE may underlie the protective effect of PE against oxidative stress observed in our study, rather than an upregulation of protein clearance. Pomegranate and its associated polyphenols were also shown to reduce amyloid loads in-vitro and in-vivo [365, 366, 453]. Enhancing the expression and level of molecular protein chaperones by polyphenols was reported as a mechanism to protect against amyloid formation and aggregation-induced toxicity. For example, resveratrol, a polyphenol found in red wine, has been shown to activate the heat-shock promoter and the expression of Hsp70 to protect the human cells from severe heat stress [454]. In addition, the green tea catechins has been found to be functionally and structurally related to the protein chaperones, suggesting that the

polyphenol itself can aid in protein folding [455]. Hence, rather than upregulating aggrephagy to reduce the levels of protein aggregates, PE may influence the expression and/or activity of molecular chaperones to reduce protein aggregation. 


\subsubsection{PE modulates mitochondrial dynamics to prime the mitochondria for autophagic degradation}

Rather than protecting against proteotoxicity by upregulating aggrephagy, our study shows that PE-induced autophagy has more relevance in mitochondrial health and fitness. Our first indication for the influence of PE on the mitochondria came from the observation that PE modulates the mitochondrial morphology, forming characteristic "donut" structures basally (Fig. 4.13a). The formation of donut-shaped mitochondria was also associated with an enhanced recruitment of autophagosomes to the mitochondria network (Fig. 4.14a and c).

Formation of donut-shaped mitochondria has been reported in several instances, including during periods of reoxygenation following acute hypoxia [426], under mild mitochondrial stress [380] and changes in osmotic pressure [271] The donut-shaped mitochondria were reported to revert back to its healthy tubular structure upon removal of the stress, or could irreversibly progress to the fragmented state if the stress persisted [380]. Fragmentation of mitochondria occurs prior to onset of mitophagy to separate the damaged mitochondria from the healthy network to only facilitate the clearance of the damaged pool [232]. The formation of the characteristic donut-shaped mitochondria under basal condition suggests that PE might induce a "primed" state in the mitochondrial network, to allow rapid fragmentation and clearance of the mitochondria upon onset of cellular stress.

Interestingly, the donut-shaped mitochondria were reported to be mild ROS generator [380]. This finding contradicts the well-established nature of the pomegranate as an antioxidant [402]. We postulate that the pro-oxidant property of PE under basal condition might be an effect peculiar to the mitochondria. Indeed, the concept of hormesis, where low doses of ROS may enhance the readiness of the cells to counteract the onset of a more aggressive cellular stress, has gained recognition as a protective mechanism the enhance the cell's resilience [456]. This is exemplified in a study which shows that ROS generated by the mitochondria is able to activate TFEB via the lysosomal-Ca ${ }^{2+}$ signaling 
to facilitate mitophagy under CCCP-induced stress [424]. Hence, we propose that formation of the donut-shaped mitochondria by PE serves dual roles. First, the donut structure allows the mitochondria to exist in an "intermediate" state that can rapidly proceed to fragmentation upon onset of mitochondrial stress. Next, the mild ROS generated by the donut structure can induce mitophagy to facilitate the clearance of the fragmented mitochondria. Indeed, our study shows that PE enhanced the recruitment of autophagosomes to the mitochondria under basal condition without mitophagy induction (Fig. 4.14). This finding further strengthens our proposal that the formation of donutshaped mitochondria by PE serves as a protective mechanism to prime the mitochondria for clearance. Further studies are required to examine if PE induces a mitochondriahormesis state under basal condition. Specific experiments involving abolishment of the reactive oxygen species (ROS) in the intracellular environment using ROS-scavengers like $\mathrm{N}$-acetylcysteine (NAC) and butylated hydroxyanisole (BHA) may be helpful to examine whether PE continues to facilitate formation of donut-shaped mitochondria with recruitment of autophagosomes under a ROS-deficient environment.

\subsubsection{PE enhances PINK1-Parkin dependent mitophagy to mitigate mitochondrial redox toxicity under mitochondrial stress}

While PE does not induce mitochondria clearance under basal condition, PE mediates clearance of damaged mitochondria upon onset of mitochondrial stress induced by CCCP (Fig. 4.15-4.17). More importantly, induction of mitochondria clearance by PE under CCCP stress seems to represent a selective autophagy phenomenon, where PINK1 and Parkin are recruited to the mitochondria network to facilitate the clearance (Fig. 4.15). The enhanced turnover of mitochondria induced by PE helped to remove the damaged and aged mitochondria (Fig. 4.16a), and alleviated CCCP-induced mitochondrial damage and ROS generation (Fig. 4.16b). Specifically, PE only influenced the turnover of damaged mitochondria but not the healthy mitochondria pool (Fig. 4.16a), reaffirming the notion that PE induces selective degradation of damaged mitochondria. 
TFEB KD abrogated the effects of PE on the autophagosomal and lysosomal compartments (Fig. 4.20) and mitochondria clearance (Fig. 4.21a and b), as well as the protective effects of PE against mitochondrial redox toxicity (Fig. 4.21c). These findings highlight PE-induced TFEB activation is important for the mitophagy upregulation to mitigate mitochondrial redox toxicity under mitochondrial stress. With this, we propose the following mechanistic model for the protective effects of PE on mitochondrial health under stress condition (Fig.5.2).

PE upregulates autophagy by inducing the release of $\mathrm{Ca}^{2+}$ from the lysosomal compartment to activate the calcineurin phosphatase. Calcineurin subsequently dephosphorylates TFEB at S142 to promote TFEB nuclear translocation. Once in the nucleus, TFEB activates the transcription of autophagy-related genes and upregulates the biogenesis of autophagosomal and lysosomal compartments. Under basal condition, PE modulates the mitochondrial dynamics and induces the formation of donut-shaped mitochondria along with recruitment of the autophagosomes to the mitochondrial network. Upon onset of mitochondrial stress, the damaged mitochondrial network rapidly fragments to facilitate clearance of mitochondria via the PINK1-Parkin mediated mitophagy pathway.

\subsubsection{Implications of PE in health and diseases}

Our finding shows that PE and its associated polyphenols protect the mitochondrial health by promoting mitophagy. Mitophagy is a key determinant of longevity, where it protects against age-dependent accumulation of deleterious mitochondria that compromises the health of the remaining mitochondrial network [423, 457-459]. Defects in mitophagy have also been observed in pathologies such as cancer, metabolic syndrome and neurodegeneration [275, 422, 460, 461]. Hence, PE-induced mitophagy poses as a viable way to improve mitochondrial health to prolong lifespan and to prevent age-related pathologies. This is supported by observations that PE supplementation in C. elegans and drosophila improves health-span and life-span by ensuring better growth, oxidative stress and infection resistance [367, 406, 407]. 
Recently, it has been demonstrated that urolithin A, a product of the metabolism of ellagitannins found in the pomegranate, is the key biomolecule contributing to the neuroprotective effects of pomegranate against mitochondrial dysfunction [367]. Urolithin A induces mitophagy and extends the lifespan in C. elegans [367]. Urolithin A-induced mitophagy was also recapitulated in mammalian muscle and intestinal cells, and shown to improve the muscle functions in rodent by preventing age-related accumulation of dysfunctional mitochondria [367]. Unlike the other polyphenols, urolithin A is the only phenolic metabolite reported thus far to be able to penetrate the blood-brain barrier [453]. Thus, the protection of the neuronal cells against mitochondrial stress by pomegranate supplementation is highly relevant to human clinical conditions.

Currently, the known methods to activate TFEB for autophagy induction involves stress triggers such as starvation, lysosomal stress and CCCP-induced mitochondrial stress [462]. Hence, PE supplementation represents a physiological way for activate TFEB for autophagy induction. Moreover, PE activates TFEB and autophagy independent of mTOR signaling, a pathway that is important for regulating a wide variety of cellular processes, including cell growth and cell metabolism [315]. Hence, compared to the current group of pharmacological drugs that targets the mTOR signaling for autophagy upregulaton to counteract diseases associated with autophagy impairment, PE supplementation represents a much favorable therapy without eliciting undesirable off-target side effects due to disruption of the mTOR signaling pathway [308]. Moreover, PE also harbors other health beneficial properties, including antioxidants, anti-inflammation and anti-microbial, making it a more attractive remedy for human diseases [372, 402]. 


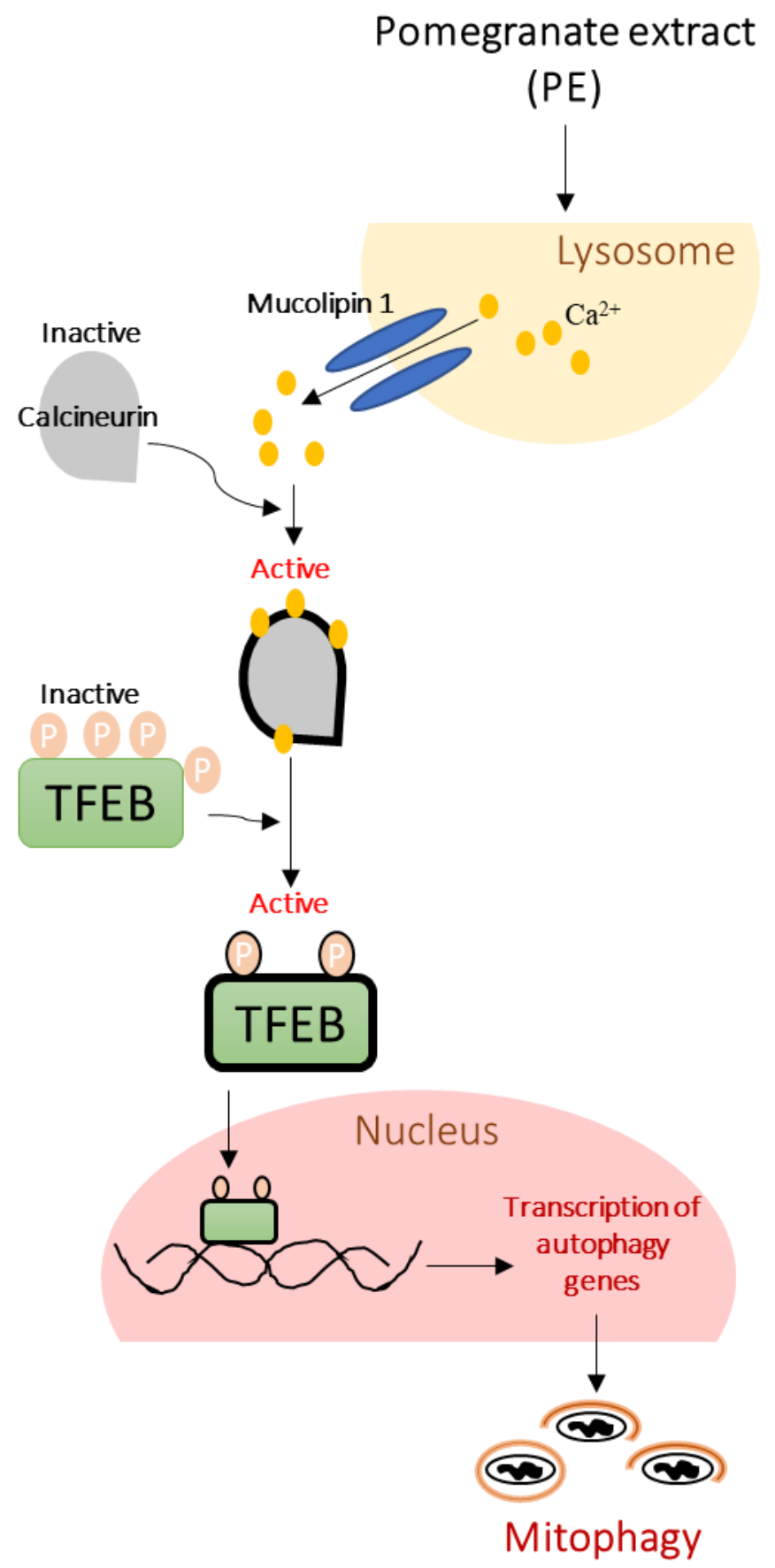


Figure 5.2 PE induces mitophagy via lysosomal-Ca ${ }^{2+}$ TFEB signaling. PE induces lysosomal $\mathrm{Ca}^{2+}$ release via mucolipin 1 channel. Localized released of $\mathrm{Ca}^{2+}$ activates the phosphatase calcineurin to dephosphorylate TFEB at S142 and activate TFEB nuclear translocation. In the nucleus, TFEB mediates transcription of autophagy and lysosomal genes, and upregulate the selective clearance of damaged mitochondria under mitochondrial stress. 


\subsection{CONCLUSION}

Selective autophagic removal of protein aggregates (aggrephagy) and damaged mitochondria (mitophagy) are crucial for preservation of proteostasis and mitochondrial homeostasis. Our study has uncovered ways where aggrephagy and mitophagy can be modulated at the molecular level via post-translational modifications, as well as at the cellular level via intake of natural products like pomegranate. In the first part of our study, we identified phosphorylation as a novel signal for regulating the basal and inducible aggrephagy behavior of Sph1. Notably, the crosstalk between phosphorylation and K63U underlie the mechanism for inducible aggrephagy. GSK3 $\beta$-mediated phosphorylation potentiates activation of E3 ligase Parkin as well as the association between Parkin and Sph1 under proteasomal stress, to facilitate addition of K63U on Sph1 for inducible aggrephagy. In the next part of our study, we identified that modulation of dietary intake via consumption of polyphenols-enriched pomegranate upregulates autophagy via transcription EB (TFEB) activation, to facilitate selective autophagic removal of the damaged mitochondria upon onset of mitochondrial stress. This process attenuates damaged mitochondria-induced redox toxicity, and preserves mitochondrial health and fitness under stress condition.

Together, our study highlights various avenues to modulate selective autophagy to target pathogenic or damaged substrates for clearance. These findings may expand the therapeutic targets for design of drugs and remedies to counteract proteotoxicity and mitochondrial dysfunction observed in aging and diseased states to promote health and longevity. 


\section{REFERENCES AND APPENDIX}

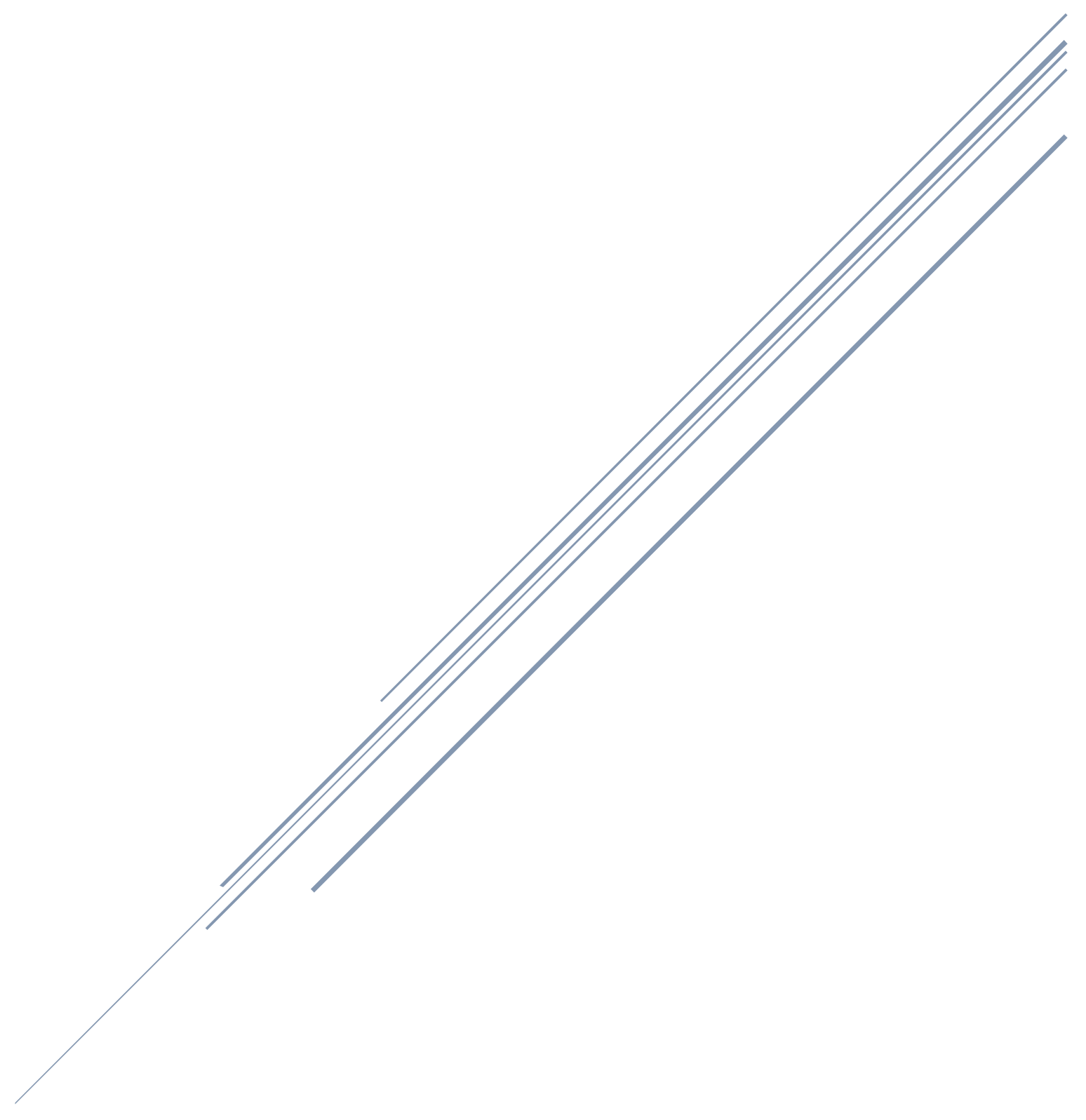




\section{REFERENCES}

1. Stoecklin, G. and B. Bukau, Telling right from wrong in life - cellular quality control. Nat Rev Mol Cell Biol, 2013. 14(10): p. 613-5.

2. Hartl, F.U., Cellular Homeostasis and Aging. Annu Rev Biochem, 2016. 85: p. 1-4.

3. Horwich, A.L., Molecular chaperones in cellular protein folding: the birth of a field. Cell, 2014. 157(2): p. 285-8.

4. Tanaka, K., The proteasome: overview of structure and functions. Proc Jpn Acad Ser B Phys Biol Sci, 2009. 85(1): p. 12-36.

5. Glick, D., S. Barth, and K.F. Macleod, Autophagy: cellular and molecular mechanisms. J Pathol, 2010. 221(1): p. 3-12.

6. De Duve, C. and R. Wattiaux, Functions of lysosomes. Annu Rev Physiol, 1966. 28: p. 435-92.

7. Mizushima, N., et al., Autophagy fights disease through cellular self-digestion. Nature, 2008. 451(7182): p. 1069-75.

8. Yang, Z. and D.J. Klionsky, Eaten alive: a history of macroautophagy. Nat Cell Biol, 2010. 12(9): p. 814-22.

9. Stolz, A., A. Ernst, and I. Dikic, Cargo recognition and trafficking in selective autophagy. Nat Cell Biol, 2014. 16(6): p. 495-501.

10. Khaminets, A., C. Behl, and I. Dikic, Ubiquitin-Dependent And Independent Signals In Selective Autophagy. Trends Cell Biol, 2016. 26(1): p. 6-16.

11. Hyttinen, J.M., et al., Clearance of misfolded and aggregated proteins by aggrephagy and implications for aggregation diseases. Ageing Res Rev, 2014. 18: p. 16-28.

12. Deng, Z., et al., Autophagy Receptors and Neurodegenerative Diseases. Trends Cell Biol, 2017. 27(7): p. 491-504. 
13. Mizumura, K., A.M. Choi, and S.W. Ryter, Emerging role of selective autophagy in human diseases. Front Pharmacol, 2014. 5: p. 244.

14. Nixon, R.A., The role of autophagy in neurodegenerative disease. Nat Med, 2013. 19(8): p. 983-97.

15. Galluzzi, L., et al., Pharmacological modulation of autophagy: therapeutic potential and persisting obstacles. Nat Rev Drug Discov, 2017. advance online publication.

16. Pallauf, K. and G. Rimbach, Autophagy, polyphenols and healthy ageing. Ageing Res Rev, 2013. 12(1): p. 237-52.

17. Testa, G., et al., Calorie restriction and dietary restriction mimetics: a strategy for improving healthy aging and longevity. Curr Pharm Des, 2014. 20(18): p. 2950-77.

18. Wong, E., et al., Molecular determinants of selective clearance of protein inclusions by autophagy. Nat Commun, 2012. 3: p. 1240.

19. Hoeijmakers, J.H., DNA damage, aging, and cancer. N Engl J Med, 2009. 361(15): p. 1475-85.

20. Sancar, A., et al., Molecular mechanisms of mammalian DNA repair and the DNA damage checkpoints. Annu Rev Biochem, 2004. 73: p. 39-85.

21. Ciccia, A. and S.J. Elledge, The DNA damage response: making it safe to play with knives. Mol Cell, 2010. 40(2): p. 179-204.

22. Inada, T., Quality control systems for aberrant mRNAs induced by aberrant translation elongation and termination. Biochim Biophys Acta, 2013. 1829(6-7): p. 634-42.

23. Schweingruber, C., et al., Nonsense-mediated mRNA decay-mechanisms of substrate mRNA recognition and degradation in mammalian cells. Biochim Biophys Acta, 2013. 1829(6-7): p. 612-23. 
24. Norbury, C.J., Cytoplasmic RNA: a case of the tail wagging the dog. Nat Rev Mol Cell Biol, 2013. 14(10): p. 643-53.

25. Marcotte, E., Evolution and the proteome: Insights into protein function from deeply conserved gene modules. The FASEB Journal, 2017. 31(1 Supplement): p. 259.2259.2.

26. Eichner, T., et al., Conformational conversion during amyloid formation at atomic resolution. Mol Cell, 2011. 41(2): p. 161-72.

27. Powers, E.T. and W.E. Balch, Diversity in the origins of proteostasis networks-a driver for protein function in evolution. Nature reviews Molecular cell biology, 2013. 14(4): p. 237-248.

28. Powers, E.T., et al., Biological and chemical approaches to diseases of proteostasis deficiency. Annu Rev Biochem, 2009. 78: p. 959-91.

29. Hipp, M.S., S.H. Park, and F.U. Hartl, Proteostasis impairment in proteinmisfolding and -aggregation diseases. Trends Cell Biol, 2014. 24(9): p. 506-14.

30. Morimoto, R.I. and A.M. Cuervo, Proteostasis and the aging proteome in health and disease. J Gerontol A Biol Sci Med Sci, 2014. 69 Suppl 1: p. S33-8.

31. Mizushima, N., Autophagy in protein and organelle turnover. Cold Spring Harb Symp Quant Biol, 2011. 76: p. 397-402.

32. Wong, E. and A.M. Cuervo, Autophagy gone awry in neurodegenerative diseases. Nat Neurosci, 2010. 13(7): p. 805-11.

33. Ryan, B.J., et al., Mitochondrial dysfunction and mitophagy in Parkinson's: from familial to sporadic disease. Trends Biochem Sci, 2015. 40(4): p. 200-10.

34. Kim, Y.E., et al., Molecular chaperone functions in protein folding and proteostasis. Annu Rev Biochem, 2013. 82: p. 323-55.

35. Mayer, M.P., Gymnastics of molecular chaperones. Mol Cell, 2010. 39(3): p. 32131. 
36. Kampinga, H.H. and E.A. Craig, The HSP70 chaperone machinery: J proteins as drivers of functional specificity. Nat Rev Mol Cell Biol, 2010. 11(8): p. 579-92.

37. Sharma, S.K., et al., The kinetic parameters and energy cost of the Hsp70 chaperone as a polypeptide unfoldase. Nat Chem Biol, 2010. 6(12): p. 914-20.

38. Frydman, J., Folding of newly translated proteins in vivo: the role of molecular chaperones. Annu Rev Biochem, 2001. 70: p. 603-47.

39. Thirumalai, D. and G.H. Lorimer, Chaperonin-mediated protein folding. Annu Rev Biophys Biomol Struct, 2001. 30: p. 245-69.

40. Reissmann, S., et al., Essential function of the built-in lid in the allosteric regulation of eukaryotic and archaeal chaperonins. Nat Struct Mol Biol, 2007. 14(5): p. 43240.

41. Behrends, C., et al., Chaperonin TRiC promotes the assembly of polyQ expansion proteins into nontoxic oligomers. Mol Cell, 2006. 23(6): p. 887-97.

42. Kitamura, A., et al., Cytosolic chaperonin prevents polyglutamine toxicity with altering the aggregation state. Nat Cell Biol, 2006. 8(10): p. 1163-70.

43. Tam, S., et al., The chaperonin TRiC controls polyglutamine aggregation and toxicity through subunit-specific interactions. Nat Cell Biol, 2006. 8(10): p. 115562.

44. McClellan, A.J., et al., Diverse cellular functions of the Hsp90 molecular chaperone uncovered using systems approaches. Cell, 2007. 131(1): p. 121-35.

45. Taipale, M., D.F. Jarosz, and S. Lindquist, HSP9O at the hub of protein homeostasis: emerging mechanistic insights. Nat Rev Mol Cell Biol, 2010. 11(7): p. $515-28$.

46. Ali, M.M., et al., Crystal structure of an Hsp90-nucleotide-p23/Sba1 closed chaperone complex. Nature, 2006. 440(7087): p. 1013-7. 
47. Shiau, A.K., et al., Structural Analysis of E. coli hsp90 reveals dramatic nucleotidedependent conformational rearrangements. Cell, 2006. 127(2): p. 329-40.

48. Scheffner, M., U. Nuber, and J.M. Huibregtse, Protein ubiquitination involving an E1-E2-E3 enzyme ubiquitin thioester cascade. Nature, 1995. 373(6509): p. 81-3.

49. Li, W.W., J. Li, and J.K. Bao, Microautophagy: lesser-known self-eating. Cell Mol Life Sci, 2012. 69(7): p. 1125-36.

50. Mijaljica, D., M. Prescott, and R.J. Devenish, Microautophagy in mammalian cells: revisiting a 40-year-old conundrum. Autophagy, 2011. 7(7): p. 673-82.

51. Roberts, P., et al., Piecemeal microautophagy of nucleus in Saccharomyces cerevisiae. Mol Biol Cell, 2003. 14(1): p. 129-41.

52. Farre, J.C., et al., Turnover of organelles by autophagy in yeast. Curr Opin Cell Biol, 2009. 21(4): p. 522-30.

53. Takikita, S., et al., Murine muscle cell models for Pompe disease and their use in studying therapeutic approaches. Mol Genet Metab, 2009. 96(4): p. 208-17.

54. Chiang, H.L. and J.F. Dice, Peptide sequences that target proteins for enhanced degradation during serum withdrawal. J Biol Chem, 1988. 263(14): p. 6797-805.

55. Dice, J.F., Selective degradation of cytosolic proteins by lysosomes. Ann N Y Acad Sci, 1992. 674: p. 58-64.

56. Agarraberes, F.A. and J.F. Dice, A molecular chaperone complex at the lysosomal membrane is required for protein translocation. J Cell Sci, 2001. 114(Pt 13): p. 2491-9.

57. Chiang, H.L., et al., A role for a 70-kilodalton heat shock protein in lysosomal degradation of intracellular proteins. Science, 1989. 246(4928): p. 382-5.

58. Cuervo, A.M. and J.F. Dice, A receptor for the selective uptake and degradation of proteins by lysosomes. Science, 1996. 273(5274): p. 501-3. 
59. Bandyopadhyay, U., et al., The chaperone-mediated autophagy receptor organizes in dynamic protein complexes at the lysosomal membrane. Mol Cell Biol, 2008. 28(18): p. 5747-63.

60. Cuervo, A.M., et al., Activation of a selective pathway of lysosomal proteolysis in rat liver by prolonged starvation. Am J Physiol, 1995. 269(5 Pt 1): p. C1200-8.

61. Kaushik, S. and A.M. Cuervo, Autophagy as a cell-repair mechanism: activation of chaperone-mediated autophagy during oxidative stress. Mol Aspects Med, 2006. 27(5-6): p. 444-54.

62. Cuervo, A.M., et al., Impaired degradation of mutant alpha-synuclein by chaperone-mediated autophagy. Science, 2004. 305(5688): p. 1292-5.

63. Orenstein, S.J., et al., Interplay of LRRK2 with chaperone-mediated autophagy. Nat Neurosci, 2013. 16(4): p. 394-406.

64. Xilouri, M., et al., Boosting chaperone-mediated autophagy in vivo mitigates alphasynuclein-induced neurodegeneration. Brain, 2013. 136(Pt 7): p. 2130-46.

65. Bauer, P.O., et al., Harnessing chaperone-mediated autophagy for the selective degradation of mutant huntingtin protein. Nat Biotechnol, 2010. 28(3): p. 256-63.

66. Feng, Y., et al., The machinery of macroautophagy. Cell Res, 2014. 24(1): p. 2441.

67. Kuma, A., et al., The role of autophagy during the early neonatal starvation period. Nature, 2004. 432(7020): p. 1032-6.

68. Ariosa, A.R. and D.J. Klionsky, Autophagy core machinery: overcoming spatial barriers in neurons. J Mol Med (Berl), 2016. 94(11): p. 1217-1227.

69. Noda, N.N., Y. Ohsumi, and F. Inagaki, ATG systems from the protein structural point of view. Chem Rev, 2009. 109(4): p. 1587-98. 
70. Popelka, H. and D.J. Klionsky, One step closer to understanding mammalian macroautophagy initiation: Interplay of 2 HORMA architectures in the ULK1 complex. Autophagy, 2015. 11(11): p. 1953-1955.

71. Lamb, C.A., T. Yoshimori, and S.A. Tooze, The autophagosome: origins unknown, biogenesis complex. Nat Rev Mol Cell Biol, 2013. 14(12): p. 759-74.

72. Karanasios, E., et al., Dynamic association of the ULK1 complex with omegasomes during autophagy induction. J Cell Sci, 2013. 126(Pt 22): p. 522438.

73. Itakura, E., et al., Beclin 1 forms two distinct phosphatidylinositol 3-kinase complexes with mammalian Atg14 and UVRAG. Mol Biol Cell, 2008. 19(12): p. 5360-72.

74. Suzuki, K., et al., Hierarchy of Atg proteins in pre-autophagosomal structure organization. Genes Cells, 2007. 12(2): p. 209-18.

75. Proikas-Cezanne, T., et al., WIPI proteins: essential Ptdlns3P effectors at the nascent autophagosome. J Cell Sci, 2015. 128(2): p. 207-17.

76. Dooley, H.C., et al., WIPI2 links LC3 conjugation with PI3P, autophagosome formation, and pathogen clearance by recruiting Atg12-5-16L1. Mol Cell, 2014. 55(2): p. 238-52.

77. Slobodkin, M.R. and Z. Elazar, The Atg8 family: multifunctional ubiquitin-like key regulators of autophagy. Essays Biochem, 2013. 55: p. 51-64.

78. Mi, N., et al., CapZ regulates autophagosomal membrane shaping by promoting actin assembly inside the isolation membrane. Nat Cell Biol, 2015. 17(9): p. 111223.

79. Orsi, A., et al., Dynamic and transient interactions of Atg9 with autophagosomes, but not membrane integration, are required for autophagy. Mol Biol Cell, 2012. 23(10): p. 1860-73. 
80. Puri, C., et al., ATG16L1 meets ATG9 in recycling endosomes: additional roles for the plasma membrane and endocytosis in autophagosome biogenesis. Autophagy, 2014. 10(1): p. 182-4.

81. Yamamoto, $\mathrm{H}_{\text {., }}$ et al., Atg9 vesicles are an important membrane source during early steps of autophagosome formation. J Cell Biol, 2012. 198(2): p. 219-33.

82. Schrader, M., et al., The different facets of organelle interplay-an overview of organelle interactions. Front Cell Dev Biol, 2015. 3: p. 56.

83. McEwan, D.G., et al., PLEKHM1 regulates autophagosome-lysosome fusion through HOPS complex and LC3/GABARAP proteins. Mol Cell, 2015. 57(1): p. 3954.

84. Zucchi, P.C. and M. Zick, Membrane fusion catalyzed by a Rab, SNAREs, and SNARE chaperones is accompanied by enhanced permeability to small molecules and by lysis. Mol Biol Cell, 2011. 22(23): p. 4635-46.

85. Itakura, E., C. Kishi-Itakura, and N. Mizushima, The hairpin-type tail-anchored SNARE syntaxin 17 targets to autophagosomes for fusion with endosomes/lysosomes. Cell, 2012. 151(6): p. 1256-69.

86. Diao, J., et al., ATG14 promotes membrane tethering and fusion of autophagosomes to endolysosomes. Nature, 2015. 520(7548): p. 563-6.

87. Yang, Z., et al., Atg22 recycles amino acids to link the degradative and recycling functions of autophagy. Mol Biol Cell, 2006. 17(12): p. 5094-104.

88. Kim, J. and K.L. Guan, Regulation of the autophagy initiating kinase ULK1 by nutrients: roles of mTORC1 and AMPK. Cell Cycle, 2011. 10(9): p. 1337-8.

89. Lee, I.H., et al., A role for the NAD-dependent deacetylase Sirt1 in the regulation of autophagy. Proc Natl Acad Sci U S A, 2008. 105(9): p. 3374-9.

90. Sardiello, M., et al., A gene network regulating lysosomal biogenesis and function. Science, 2009. 325(5939): p. 473-7. 
91. Settembre, C., et al., TFEB links autophagy to lysosomal biogenesis. Science, 2011. 332(6036): p. 1429-33.

92. Loos, B., et al., The variability of autophagy and cell death susceptibility: Unanswered questions. Autophagy, 2013. 9(9): p. 1270-85.

93. Bhaskar, P.T. and N. Hay, The two TORCs and Akt. Dev Cell, 2007. 12(4): p. 487502.

94. Schmelzle, T. and M.N. Hall, TOR, a central controller of cell growth. Cell, 2000. 103(2): p. 253-62.

95. Noda, T. and Y. Ohsumi, Tor, a phosphatidylinositol kinase homologue, controls autophagy in yeast. J Biol Chem, 1998. 273(7): p. 3963-6.

96. Kim, J., et al., AMPK and mTOR regulate autophagy through direct phosphorylation of Ulk1. Nat Cell Biol, 2011. 13(2): p. 132-41.

97. Corradetti, M.N. and K.L. Guan, Upstream of the mammalian target of rapamycin: do all roads pass through mTOR? Oncogene, 2006. 25(48): p. 6347-60.

98. Zhang, S., et al., Novel metabolic and physiological functions of branched chain amino acids: a review. J Anim Sci Biotechnol, 2017. 8: p. 10.

99. Long, X., et al., Rheb binding to mammalian target of rapamycin (mTOR) is regulated by amino acid sufficiency. J Biol Chem, 2005. 280(25): p. 23433-6.

100. Miyazaki, M., J.J. McCarthy, and K.A. Esser, Insulin like growth factor-1-induced phosphorylation and altered distribution of tuberous sclerosis complex (TSC)1/TSC2 in C2C12 myotubes. Febs j, 2010. 277(9): p. 2180-91.

101. Egan, D., et al., The autophagy initiating kinase ULK1 is regulated via opposing phosphorylation by AMPK and mTOR. Autophagy, 2011. 7(6): p. 643-4.

102. Steingrimsson, E., N.G. Copeland, and N.A. Jenkins, Melanocytes and the microphthalmia transcription factor network. Annu Rev Genet, 2004. 38: p. 365411. 
103. Palmieri, M., et al., Characterization of the CLEAR network reveals an integrated control of cellular clearance pathways. Hum Mol Genet, 2011. 20(19): p. 3852-66.

104. Settembre, C. and D.L. Medina, TFEB and the CLEAR network. Methods Cell Biol, 2015. 126: p. 45-62.

105. Nezich, C.L., et al., MiT/TFE transcription factors are activated during mitophagy downstream of Parkin and Atg5. J Cell Biol, 2015. 210(3): p. 435-50.

106. Settembre, C., et al., TFEB controls cellular lipid metabolism through a starvationinduced autoregulatory loop. Nat Cell Biol, 2013. 15(6): p. 647-58.

107. Parenti, G., G. Andria, and A. Ballabio, Lysosomal storage diseases: from pathophysiology to therapy. Annu Rev Med, 2015. 66: p. 471-86.

108. Rega, L.R., et al., Activation of the transcription factor EB rescues lysosomal abnormalities in cystinotic kidney cells. Kidney Int, 2016. 89(4): p. 862-73.

109. Song, W., et al., TFEB regulates lysosomal proteostasis. Hum Mol Genet, 2013. 22(10): p. 1994-2009.

110. Spampanato, C., et al., Transcription factor $E B$ (TFEB) is a new therapeutic target for Pompe disease. EMBO Mol Med, 2013. 5(5): p. 691-706.

111. Decressac, M., et al., TFEB-mediated autophagy rescues midbrain dopamine neurons from alpha-synuclein toxicity. Proc Natl Acad Sci U S A, 2013. 110(19): p. E1817-26.

112. Kilpatrick, K., et al., Genetic and chemical activation of TFEB mediates clearance of aggregated alpha-synuclein. PLoS One, 2015. 10(3): p. e0120819.

113. Tsunemi, T., et al., PGC-1alpha rescues Huntington's disease proteotoxicity by preventing oxidative stress and promoting TFEB function. Sci Transl Med, 2012. 4(142): p. 142 ra97.

114. Polito, V.A., et al., Selective clearance of aberrant tau proteins and rescue of neurotoxicity by transcription factor EB. EMBO Mol Med, 2014. 6(9): p. 1142-60. 
115. Xiao, Q., et al., Enhancing astrocytic lysosome biogenesis facilitates Abeta clearance and attenuates amyloid plaque pathogenesis. J Neurosci, 2014. 34(29): p. 9607-20.

116. Xiao, Q., et al., Neuronal-Targeted TFEB Accelerates Lysosomal Degradation of APP, Reducing Abeta Generation and Amyloid Plaque Pathogenesis. J Neurosci, 2015. 35(35): p. 12137-51.

117. Martina, J.A., et al., MTORC1 functions as a transcriptional regulator of autophagy by preventing nuclear transport of TFEB. Autophagy, 2012. 8(6): p. 903-14.

118. Roczniak-Ferguson, A., et al., The transcription factor TFEB links mTORC1 signaling to transcriptional control of lysosome homeostasis. Sci Signal, 2012. 5(228): p. ra42.

119. Settembre, C., et al., A lysosome-to-nucleus signalling mechanism senses and regulates the lysosome via mTOR and TFEB. Embo j, 2012. 31(5): p. 1095-108.

120. Medina, D.L., et al., Lysosomal calcium signalling regulates autophagy through calcineurin and TFEB. Nat Cell Biol, 2015. 17(3): p. 288-99.

121. Fimia, G.M., G. Kroemer, and M. Piacentini, Molecular mechanisms of selective autophagy. Cell Death Differ, 2013. 20(1): p. 1-2.

122. Zhang, T. and S. Ghaemmaghami, Global analysis of cellular protein flux quantifies the selectivity of basal autophagy. Autophagy, 2016. 12(8): p. 1411-2.

123. Hara, T., et al., Suppression of basal autophagy in neural cells causes neurodegenerative disease in mice. Nature, 2006. 441(7095): p. 885-9.

124. Komatsu, M., et al., Loss of autophagy in the central nervous system causes neurodegeneration in mice. Nature, 2006. 441(7095): p. 880-4.

125. Takamura, A., et al., Autophagy-deficient mice develop multiple liver tumors. Genes Dev, 2011. 25(8): p. 795-800. 
126. Johansen, T. and T. Lamark, Selective autophagy mediated by autophagic adapter proteins. Autophagy, 2011. 7(3): p. 279-296.

127. Rogov, V., et al., Interactions between autophagy receptors and ubiquitin-like proteins form the molecular basis for selective autophagy. Mol Cell, 2014. 53(2): p. 167-78.

128. Singh, R., et al., Autophagy regulates lipid metabolism. Nature, 2009. 458(7242): p. 1131-5.

129. Kraft, C., et al., Mature ribosomes are selectively degraded upon starvation by an autophagy pathway requiring the Ubp3p/Bre5p ubiquitin protease. Nat Cell Biol, 2008. 10(5): p. 602-10.

130. Pohl, C. and S. Jentsch, Midbody ring disposal by autophagy is a post-abscission event of cytokinesis. Nat Cell Biol, 2009. 11(1): p. 65-70.

131. Marshall, R.S., et al., Autophagic Degradation of the 26S Proteasome Is Mediated by the Dual ATG8/Ubiquitin Receptor RPN10 in Arabidopsis. Mol Cell, 2015. 58(6): p. 1053-66.

132. Noda, N.N., et al., Structural basis of target recognition by Atg8/LC3 during selective autophagy. Genes Cells, 2008. 13(12): p. 1211-8.

133. Pankiv, S., et al., p62/SQSTM1 binds directly to Atg8/LC3 to facilitate degradation of ubiquitinated protein aggregates by autophagy. J Biol Chem, 2007. 282(33): p. 24131-45.

134. Bartlett, A.I. and S.E. Radford, An expanding arsenal of experimental methods yields an explosion of insights into protein folding mechanisms. Nat Struct Mol Biol, 2009. 16(6): p. 582-8.

135. Dill, K.A., et al., Principles of protein folding--a perspective from simple exact models. Protein Sci, 1995. 4(4): p. 561-602. 
136. Knowles, T.P., M. Vendruscolo, and C.M. Dobson, The amyloid state and its association with protein misfolding diseases. Nat Rev Mol Cell Biol, 2014. 15(6): p. 384-96.

137. Lee, S.J., et al., Cell-to-cell transmission of non-prion protein aggregates. Nat Rev Neurol, 2010. 6(12): p. 702-6.

138. Polymenidou, M. and D.W. Cleveland, The seeds of neurodegeneration: prion-like spreading in ALS. Cell, 2011. 147(3): p. 498-508.

139. Collinge, J. and A.R. Clarke, A general model of prion strains and their pathogenicity. Science, 2007. 318(5852): p. 930-6.

140. Ross, C.A. and M.A. Poirier, Protein aggregation and neurodegenerative disease. Nat Med, 2004. 10 Suppl: p. S10-7.

141. Caughey, B. and P.T. Lansbury, Protofibrils, pores, fibrils, and neurodegeneration: separating the responsible protein aggregates from the innocent bystanders. Annu Rev Neurosci, 2003. 26: p. 267-98.

142. Walsh, D.M. and D.J. Selkoe, Oligomers on the brain: the emerging role of soluble protein aggregates in neurodegeneration. Protein Pept Lett, 2004. 11(3): p. 21328.

143. Baglioni, S., et al., Prefibrillar amyloid aggregates could be generic toxins in higher organisms. J Neurosci, 2006. 26(31): p. 8160-7.

144. Haass, C. and D.J. Selkoe, Soluble protein oligomers in neurodegeneration: lessons from the Alzheimer's amyloid beta-peptide. Nat Rev Mol Cell Biol, 2007. 8(2): p. 101-12.

145. Winner, B., et al., In vivo demonstration that alpha-synuclein oligomers are toxic. Proc Natl Acad Sci U S A, 2011. 108(10): p. 4194-9.

146. Campioni, S., et al., A causative link between the structure of aberrant protein oligomers and their toxicity. Nat Chem Biol, 2010. 6(2): p. 140-7. 
147. Cremades, N., et al., Direct observation of the interconversion of normal and toxic forms of alpha-synuclein. Cell, 2012. 149(5): p. 1048-59.

148. Johnston, J.A., C.L. Ward, and R.R. Kopito, Aggresomes: a cellular response to misfolded proteins. J Cell Biol, 1998. 143(7): p. 1883-98.

149. Miller, S.B., A. Mogk, and B. Bukau, Spatially Organized Aggregation of Misfolded Proteins as Cellular Stress Defense Strategy. J Mol Biol, 2015. 427(7): p. 15641574.

150. Ventura, S. and A. Villaverde, Protein quality in bacterial inclusion bodies. Trends Biotechnol, 2006. 24(4): p. 179-85.

151. Sontag, E.M., W.I. Vonk, and J. Frydman, Sorting out the trash: the spatial nature of eukaryotic protein quality control. Curr Opin Cell Biol, 2014. 26: p. 139-46.

152. Kaganovich, D., R. Kopito, and J. Frydman, Misfolded proteins partition between two distinct quality control compartments. Nature, 2008. 454(7208): p. 1088-95.

153. Tyedmers, J., A. Mogk, and B. Bukau, Cellular strategies for controlling protein aggregation. Nat Rev Mol Cell Biol, 2010. 11(11): p. 777-88.

154. Markossian, K.A. and B.I. Kurganov, Protein folding, misfolding, and aggregation. Formation of inclusion bodies and aggresomes. Biochemistry (Mosc), 2004. 69(9): p. 971-84.

155. Kopito, R.R., Aggresomes, inclusion bodies and protein aggregation. Trends Cell Biol, 2000. 10(12): p. 524-30.

156. Corboy, M.J., P.J. Thomas, and W.C. Wigley, Aggresome formation. Methods Mol Biol, 2005. 301: p. 305-27.

157. Miller, S.B., et al., Compartment-specific aggregases direct distinct nuclear and cytoplasmic aggregate deposition. EMBO J, 2015. 34(6): p. 778-97.

158. Bagola, K. and T. Sommer, Protein quality control: on IPODs and other JUNQ. Curr Biol, 2008. 18(21): p. R1019-21. 
159. Specht, S., et al., Hsp42 is required for sequestration of protein aggregates into deposition sites in Saccharomyces cerevisiae. J Cell Biol, 2011. 195(4): p. 617-29.

160. Escusa-Toret, S., W.I. Vonk, and J. Frydman, Spatial sequestration of misfolded proteins by a dynamic chaperone pathway enhances cellular fitness during stress. Nat Cell Biol, 2013. 15(10): p. 1231-43.

161. McNaught, K.S., et al., Aggresome-related biogenesis of Lewy bodies. Eur J Neurosci, 2002. 16(11): p. 2136-48.

162. Zaarur, N., et al., Proteasome failure promotes positioning of lysosomes around the aggresome via local block of microtubule-dependent transport. Mol Cell Biol, 2014. 34(7): p. 1336-48.

163. Olzmann, J.A., et al., Parkin-mediated K63-linked polyubiquitination targets misfolded DJ-1 to aggresomes via binding to HDAC6. J Cell Biol, 2007. 178(6): p. 1025-38.

164. Kirkin, V., et al., A role for NBR1 in autophagosomal degradation of ubiquitinated substrates. Mol Cell, 2009. 33(4): p. 505-16.

165. Lim, K.L., V.L. Dawson, and T.M. Dawson, Parkin-mediated lysine 63-linked polyubiquitination: a link to protein inclusions formation in Parkinson's and other conformational diseases? Neurobiol Aging, 2006. 27(4): p. 524-9.

166. Moscat, J., M.T. Diaz-Meco, and M.W. Wooten, Signal integration and diversification through the p62 scaffold protein. Trends Biochem Sci, 2007. 32(2): p. $95-100$.

167. Burnett, B.G. and R.N. Pittman, The polyglutamine neurodegenerative protein ataxin 3 regulates aggresome formation. Proc Natl Acad Sci U S A, 2005. 102(12): p. $4330-5$.

168. Ouyang, H., et al., Protein aggregates are recruited to aggresome by histone deacetylase 6 via unanchored ubiquitin C termini. J Biol Chem, 2012. 287(4): p. 2317-27. 
169. Lamark, T., et al., NBR1 and p62 as cargo receptors for selective autophagy of ubiquitinated targets. Cell Cycle, 2009. 8(13): p. 1986-90.

170. Wooten, M.W., et al., Essential role of sequestosome 1/p62 in regulating accumulation of Lys63-ubiquitinated proteins. J Biol Chem, 2008. 283(11): p. 6783-9.

171. Walinda, E., et al., Solution structure of the ubiquitin-associated (UBA) domain of human autophagy receptor NBR1 and its interaction with ubiquitin and polyubiquitin. J Biol Chem, 2014. 289(20): p. 13890-902.

172. Itakura, E. and N. Mizushima, p62 Targeting to the autophagosome formation site requires self-oligomerization but not LC3 binding. J Cell Biol, 2011. 192(1): p. 1727.

173. Duran, A., et al., p62 is a key regulator of nutrient sensing in the mTORC1 pathway. Mol Cell, 2011. 44(1): p. 134-46.

174. Lee, J.Y., et al., HDAC6 controls autophagosome maturation essential for ubiquitin-selective quality-control autophagy. EMBO J, 2010. 29(5): p. 969-80.

175. Yan, J., et al., SQSTM1/p62 interacts with HDAC6 and regulates deacetylase activity. PLoS One, 2013. 8(9): p. e76016.

176. Hao, R., et al., Proteasomes activate aggresome disassembly and clearance by producing unanchored ubiquitin chains. Mol Cell, 2013. 51(6): p. 819-28.

177. Nanduri, P., et al., Chaperone-mediated 26S Proteasome Remodeling Facilitates Free K63 Ubiquitin Chain Production and Aggresome Clearance. J Biol Chem, 2015.

178. Matsumoto, G., et al., Serine 403 phosphorylation of p62/SQSTM1 regulates selective autophagic clearance of ubiquitinated proteins. Mol Cell, 2011. 44(2): p. 279-89. 
179. Nicot, A.S., et al., Phosphorylation of NBR1 by GSK3 modulates protein aggregation. Autophagy, 2014. 10(6): p. 1036-53.

180. Korac, J., et al., Ubiquitin-independent function of optineurin in autophagic clearance of protein aggregates. J Cell Sci, 2013. 126(Pt 2): p. 580-92.

181. Wong, E.S., et al., Autophagy-mediated clearance of aggresomes is not a universal phenomenon. Hum Mol Genet, 2008. 17(16): p. 2570-82.

182. Tanik, S.A., et al., Lewy body-like alpha-synuclein aggregates resist degradation and impair macroautophagy. J Biol Chem, 2013. 288(21): p. 15194-210.

183. $\mathrm{Du}, \mathrm{Y}$., et al., Age-associated oxidative damage to the $\mathrm{p} 62$ promoter: implications for Alzheimer disease. Free Radic Biol Med, 2009. 46(4): p. 492-501.

184. Wakabayashi, K., et al., Synphilin-1 is present in Lewy bodies in Parkinson's disease. Ann Neurol, 2000. 47(4): p. 521-3.

185. Moore, D.J., et al., Molecular pathophysiology of Parkinson's disease. Annu Rev Neurosci, 2005. 28: p. 57-87.

186. Engelender, S., et al., Synphilin-1 associates with alpha-synuclein and promotes the formation of cytosolic inclusions. Nat Genet, 1999. 22(1): p. 110-4.

187. Murray, I.J., et al., Synphilin in normal human brains and in synucleinopathies: studies with new antibodies. Acta Neuropathol, 2003. 105(2): p. 177-84.

188. Ribeiro, C.S., et al., Synphilin-1 is developmentally localized to synaptic terminals, and its association with synaptic vesicles is modulated by alpha-synuclein. $\mathrm{J}$ Biol Chem, 2002. 277(26): p. 23927-33.

189. Krygowska-Wajs, A., et al., Increased synphilin-1 expression in human elderly brains with substantia nigra Marinesco bodies. Pharmacol Rep, 2008. 60(6): p. 914-24.

190. Hernandez-Vargas, R., et al., Synphilin suppresses alpha-synuclein neurotoxicity in a Parkinson's disease Drosophila model. Genesis, 2011. 49(5): p. 392-402. 
191. Smith, W.W., et al., Synphilin-1 attenuates neuronal degeneration in the A53T alpha-synuclein transgenic mouse model. Hum Mol Genet, 2010. 19(11): p. 208798.

192. Tanaka, M., et al., Aggresomes formed by alpha-synuclein and synphilin-1 are cytoprotective. J Biol Chem, 2004. 279(6): p. 4625-31.

193. Casadei, N., et al., Overexpression of synphilin-1 promotes clearance of soluble and misfolded alpha-synuclein without restoring the motor phenotype in aged A30P transgenic mice. Hum Mol Genet, 2014. 23(3): p. 767-81.

194. Swinnen, E., et al., Aggresome formation and segregation of inclusions influence toxicity of alpha-synuclein and synphilin-1 in yeast. Biochem Soc Trans, 2011. 39(5): p. 1476-81.

195. Marx, F.P., et al., Identification and functional characterization of a novel R621C mutation in the synphilin-1 gene in Parkinson's disease. Hum Mol Genet, 2003. 12(11): p. 1223-31.

196. Ito, T., et al., Dorfin localizes to Lewy bodies and ubiquitylates synphilin-1. J Biol Chem, 2003. 278(31): p. 29106-14.

197. Nagano, Y., et al., Siah-1 facilitates ubiquitination and degradation of synphilin-1. J Biol Chem, 2003. 278(51): p. 51504-14.

198. Lim, K.L., et al., Parkin mediates nonclassical, proteasomal-independent ubiquitination of synphilin-1: implications for Lewy body formation. J Neurosci, 2005. 25(8): p. 2002-9.

199. Chung, K.K., et al., Parkin ubiquitinates the alpha-synuclein-interacting protein, synphilin-1: implications for Lewy-body formation in Parkinson disease. Nat Med, 2001. 7(10): p. 1144-50.

200. Lim, G.G., et al., Proteasome inhibition promotes Parkin-Ubc13 interaction and lysine 63-linked ubiquitination. PLoS One, 2013. 8(9): p. e73235. 
201. Lee, G., et al., Casein kinase II-mediated phosphorylation regulates alphasynuclein/synphilin-1 interaction and inclusion body formation. J Biol Chem, 2004. 279(8): p. 6834-9.

202. Avraham, E., et al., Glycogen synthase kinase 3beta modulates synphilin-1 ubiquitylation and cellular inclusion formation by SIAH: implications for proteasomal function and Lewy body formation. J Biol Chem, 2005. 280(52): p. 42877-86.

203. Liu, J., et al., Synphilin-1 attenuates mutant LRRK2-induced neurodegeneration in Parkinson's disease models. Hum Mol Genet, 2016. 25(4): p. 672-80.

204. Szargel, R., et al., The PINK1, synphilin-1 and SIAH-1 complex constitutes a novel mitophagy pathway. Hum Mol Genet, 2016. 25(16): p. 3476-3490.

205. Zaarur, N., et al., Triggering aggresome formation. Dissecting aggresometargeting and aggregation signals in synphilin 1. J Biol Chem, 2008. 283(41): $\mathrm{p}$. 27575-84.

206. Szargel, R., R. Rott, and S. Engelender, Synphilin-1 isoforms in Parkinson's disease: regulation by phosphorylation and ubiquitylation. Cell Mol Life Sci, 2008. 65(1): p. 80-8.

207. Zhu, X.H., et al., Quantitative imaging of energy expenditure in human brain. Neuroimage, 2012. 60(4): p. 2107-17.

208. Friedman, J.R. and J. Nunnari, Mitochondrial form and function. Nature, 2014. 505(7483): p. 335-43.

209. Newmeyer, D.D. and S. Ferguson-Miller, Mitochondria: releasing power for life and unleashing the machineries of death. Cell, 2003. 112(4): p. 481-90.

210. Cai, Q., M.L. Davis, and Z.H. Sheng, Regulation of axonal mitochondrial transport and its impact on synaptic transmission. Neurosci Res, 2011. 70(1): p. 9-15. 
211. Chan, D.C., Mitochondria: dynamic organelles in disease, aging, and development. Cell, 2006. 125(7): p. 1241-52.

212. Wallace, D.C., A mitochondrial paradigm of metabolic and degenerative diseases, aging, and cancer: a dawn for evolutionary medicine. Annu Rev Genet, 2005. 39: p. 359-407.

213. Parsons, M.J. and D.R. Green, Mitochondria in cell death. Essays Biochem, 2010. 47: p. 99-114.

214. Palikaras, K. and N. Tavernarakis, Mitochondrial homeostasis: the interplay between mitophagy and mitochondrial biogenesis. Exp Gerontol, 2014. 56: p. 1828.

215. Taanman, J.W., The mitochondrial genome: structure, transcription, translation and replication. Biochim Biophys Acta, 1999. 1410(2): p. 103-23.

216. Wenz, L.S., et al., Sam37 is crucial for formation of the mitochondrial TOM-SAM supercomplex, thereby promoting beta-barrel biogenesis. J Cell Biol, 2015. 210(7): p. 1047-54.

217. Eliyahu, E., et al., Tom20 mediates localization of mRNAs to mitochondria in a translation-dependent manner. Mol Cell Biol, 2010. 30(1): p. 284-94.

218. Lesnik, C., et al., OM14 is a mitochondrial receptor for cytosolic ribosomes that supports co-translational import into mitochondria. Nat Commun, 2014. 5: p. 5711.

219. Scarpulla, R.C., Transcriptional paradigms in mammalian mitochondrial biogenesis and function. Physiol Rev, 2008. 88(2): p. 611-38.

220. Dominy, J.E. and P. Puigserver, Mitochondrial biogenesis through activation of nuclear signaling proteins. Cold Spring Harb Perspect Biol, 2013. 5(7).

221. Satoh, J., N. Kawana, and Y. Yamamoto, Pathway Analysis of ChIP-Seq-Based NRF1 Target Genes Suggests a Logical Hypothesis of their Involvement in the 
Pathogenesis of Neurodegenerative Diseases. Gene Regul Syst Bio, 2013. 7: p. 139-52.

222. Biswas, M. and J.Y. Chan, Role of Nrf1 in antioxidant response element-mediated gene expression and beyond. Toxicol Appl Pharmacol, 2010. 244(1): p. 16-20.

223. Yang, Z.F., et al., GABP transcription factor (nuclear respiratory factor 2) is required for mitochondrial biogenesis. Mol Cell Biol, 2014. 34(17): p. 3194-201.

224. Birkenfeld, A.L., et al., Deletion of the mammalian INDY homolog mimics aspects of dietary restriction and protects against adiposity and insulin resistance in mice. Cell Metab, 2011. 14(2): p. 184-95.

225. Canto, C., et al., AMPK regulates energy expenditure by modulating NAD+ metabolism and SIRT1 activity. Nature, 2009. 458(7241): p. 1056-60.

226. Wu, H., et al., Regulation of mitochondrial biogenesis in skeletal muscle by CaMK. Science, 2002. 296(5566): p. 349-52.

227. Wright, D.C., et al., Calcium induces increases in peroxisome proliferator-activated receptor gamma coactivator-1alpha and mitochondrial biogenesis by a pathway leading to p38 mitogen-activated protein kinase activation. J Biol Chem, 2007. 282(26): p. 18793-9.

228. Srivastava, S., et al., PGC-1alpha/beta induced expression partially compensates for respiratory chain defects in cells from patients with mitochondrial disorders. Hum Mol Genet, 2009. 18(10): p. 1805-12.

229. Langer, T., et al., $A A A$ proteases of mitochondria: quality control of membrane proteins and regulatory functions during mitochondrial biogenesis. Biochem Soc Trans, 2001. 29(Pt 4): p. 431-6.

230. Tanaka, A., et al., Proteasome and p97 mediate mitophagy and degradation of mitofusins induced by Parkin. J Cell Biol, 2010. 191(7): p. 1367-80. 
231. Sugiura, A., et al., A new pathway for mitochondrial quality control: mitochondrialderived vesicles. Embo j, 2014. 33(19): p. 2142-56.

232. Chan, D.C., Fusion and fission: interlinked processes critical for mitochondrial health. Annu Rev Genet, 2012. 46: p. 265-87.

233. Okamoto, K. and J.M. Shaw, Mitochondrial morphology and dynamics in yeast and multicellular eukaryotes. Annu Rev Genet, 2005. 39: p. 503-36.

234. Mortiboys, H., et al., Mitochondrial function and morphology are impaired in parkinmutant fibroblasts. Ann Neurol, 2008. 64(5): p. 555-65.

235. Hoppins, S., L. Lackner, and J. Nunnari, The machines that divide and fuse mitochondria. Annu Rev Biochem, 2007. 76: p. 751-80.

236. Smirnova, E., et al., Dynamin-related protein Drp1 is required for mitochondrial division in mammalian cells. Mol Biol Cell, 2001. 12(8): p. 2245-56.

237. Chen, $\mathrm{H}$., et al., Mitofusins Mfn1 and Mfn2 coordinately regulate mitochondrial fusion and are essential for embryonic development. J Cell Biol, 2003. 160(2): p. 189-200.

238. Ishihara, N., Y. Eura, and K. Mihara, Mitofusin 1 and 2 play distinct roles in mitochondrial fusion reactions via GTPase activity. J Cell Sci, 2004. 117(Pt 26): p. 6535-46.

239. Cipolat, S., et al., OPA1 requires mitofusin 1 to promote mitochondrial fusion. Proc Natl Acad Sci U S A, 2004. 101(45): p. 15927-32.

240. Song, Z., et al., Mitofusins and OPA1 mediate sequential steps in mitochondrial membrane fusion. Mol Biol Cell, 2009. 20(15): p. 3525-32.

241. Olichon, A., et al., Loss of OPA1 perturbates the mitochondrial inner membrane structure and integrity, leading to cytochrome c release and apoptosis. J Biol Chem, 2003. 278(10): p. 7743-6. 
242. Gegg, M.E., et al., Mitofusin 1 and mitofusin 2 are ubiquitinated in a PINK1/parkindependent manner upon induction of mitophagy. Hum Mol Genet, 2010. 19(24): p. 4861-70.

243. Valente, E.M., et al., Hereditary early-onset Parkinson's disease caused by mutations in PINK1. Science, 2004. 304(5674): p. 1158-60.

244. Kitada, T., et al., Mutations in the parkin gene cause autosomal recessive juvenile parkinsonism. Nature, 1998. 392(6676): p. 605-8.

245. Meissner, C., et al., The mitochondrial intramembrane protease PARL cleaves human Pink1 to regulate Pink1 trafficking. J Neurochem, 2011. 117(5): p. 856-67.

246. Greene, A.W., et al., Mitochondrial processing peptidase regulates PINK1 processing, import and Parkin recruitment. EMBO Rep, 2012. 13(4): p. 378-85.

247. Deas, E., et al., PINK1 cleavage at position A103 by the mitochondrial protease PARL. Hum Mol Genet, 2011. 20(5): p. 867-79.

248. Lazarou, M., et al., Role of PINK1 binding to the TOM complex and alternate intracellular membranes in recruitment and activation of the E3 ligase Parkin. Dev Cell, 2012. 22(2): p. 320-33.

249. Okatsu, K., et al., PINK1 autophosphorylation upon membrane potential dissipation is essential for Parkin recruitment to damaged mitochondria. Nat Commun, 2012. 3: p. 1016.

250. Riley, B.E., et al., Structure and function of Parkin E3 ubiquitin ligase reveals aspects of RING and HECT ligases. Nat Commun, 2013. 4: p. 1982.

251. Kazlauskaite, A., et al., Parkin is activated by PINK1-dependent phosphorylation of ubiquitin at Ser65. Biochem J, 2014. 460(1): p. 127-39.

252. Caulfield, T.R., et al., Phosphorylation by PINK1 releases the UBL domain and initializes the conformational opening of the E3 ubiquitin ligase Parkin. PLoS Comput Biol, 2014. 10(11): p. e1003935. 
253. Kane, L.A., et al., PINK1 phosphorylates ubiquitin to activate Parkin E3 ubiquitin ligase activity. J Cell Biol, 2014. 205(2): p. 143-53.

254. Chen, Y. and G.W. Dorn, 2nd, PINK1-phosphorylated mitofusin 2 is a Parkin receptor for culling damaged mitochondria. Science, 2013. 340(6131): p. 471-5.

255. Geisler, S., et al., PINK1/Parkin-mediated mitophagy is dependent on VDAC1 and p62/SQSTM1. Nat Cell Biol, 2010. 12(2): p. 119-31.

256. Youle, R.J. and D.P. Narendra, Mechanisms of mitophagy. Nat Rev Mol Cell Biol, 2011. 12(1): p. 9-14.

257. Narendra, D., et al., p62/SQSTM1 is required for Parkin-induced mitochondrial clustering but not mitophagy; VDAC1 is dispensable for both. Autophagy, 2010. 6(8): p. 1090-106.

258. Van Humbeeck, C., et al., Parkin interacts with Ambra1 to induce mitophagy. J Neurosci, 2011. 31(28): p. 10249-61.

259. Michiorri, S., et al., The Parkinson-associated protein PINK1 interacts with Beclin1 and promotes autophagy. Cell Death Differ, 2010. 17(6): p. 962-74.

260. Sheng, Z.H. and Q. Cai, Mitochondrial transport in neurons: impact on synaptic homeostasis and neurodegeneration. Nat Rev Neurosci, 2012. 13(2): p. 77-93.

261. Cai, Q., et al., Spatial parkin translocation and degradation of damaged mitochondria via mitophagy in live cortical neurons. Curr Biol, 2012. 22(6): p. 54552.

262. Maday, S. and E.L. Holzbaur, Compartment-Specific Regulation of Autophagy in Primary Neurons. J Neurosci, 2016. 36(22): p. 5933-45.

263. Maday, S., Mechanisms of neuronal homeostasis: Autophagy in the axon. Brain Res, 2016. 1649(Pt B): p. 143-150.

264. Melrose, H.L., et al., Parkinson's disease: a rethink of rodent models. Exp Brain Res, 2006. 173(2): p. 196-204. 
265. Ashrafi, G. and T.L. Schwarz, The pathways of mitophagy for quality control and clearance of mitochondria. Cell Death Differ, 2013. 20(1): p. 31-42.

266. Rikka, S., et al., Bnip3 impairs mitochondrial bioenergetics and stimulates mitochondrial turnover. Cell Death Differ, 2011. 18(4): p. 721-31.

267. Hanna, R.A., et al., Microtubule-associated protein 1 light chain 3 (LC3) interacts with Bnip3 protein to selectively remove endoplasmic reticulum and mitochondria via autophagy. J Biol Chem, 2012. 287(23): p. 19094-104.

268. Novak, I., et al., Nix is a selective autophagy receptor for mitochondrial clearance. EMBO Rep, 2010. 11(1): p. 45-51.

269. Zhu, Y., et al., Modulation of serines 17 and 24 in the LC3-interacting region of Bnip3 determines pro-survival mitophagy versus apoptosis. J Biol Chem, 2013. 288(2): p. 1099-113.

270. Wu, W., et al., ULK1 translocates to mitochondria and phosphorylates FUNDC1 to regulate mitophagy. EMBO Rep, 2014. 15(5): p. 566-75.

271. Liu, L., et al., Mitochondrial outer-membrane protein FUNDC1 mediates hypoxiainduced mitophagy in mammalian cells. Nat Cell Biol, 2012. 14(2): p. 177-85.

272. Lazarou, M., et al., The ubiquitin kinase PINK1 recruits autophagy receptors to induce mitophagy. Nature, 2015. 524(7565): p. 309-14.

273. Chu, C.T., et al., Cardiolipin externalization to the outer mitochondrial membrane acts as an elimination signal for mitophagy in neuronal cells. Nat Cell Biol, 2013. 15(10): p. 1197-205.

274. Sentelle, R.D., et al., Ceramide targets autophagosomes to mitochondria and induces lethal mitophagy. Nat Chem Biol, 2012. 8(10): p. 831-8.

275. Lionaki, E., et al., Mitochondria, autophagy and age-associated neurodegenerative diseases: New insights into a complex interplay. Biochim Biophys Acta, 2015. 1847(11): p. 1412-23. 
276. Jager, S., et al., AMP-activated protein kinase (AMPK) action in skeletal muscle via direct phosphorylation of PGC-1alpha. Proc Natl Acad Sci U S A, 2007. 104(29): p. 12017-22.

277. Price, N.L., et al., SIRT1 is required for AMPK activation and the beneficial effects of resveratrol on mitochondrial function. Cell Metab, 2012. 15(5): p. 675-90.

278. Kuroda, Y., et al., Parkin enhances mitochondrial biogenesis in proliferating cells. Hum Mol Genet, 2006. 15(6): p. 883-95.

279. Shin, J.H., et al., PARIS (ZNF746) repression of PGC-1alpha contributes to neurodegeneration in Parkinson's disease. Cell, 2011. 144(5): p. 689-702.

280. Strnad, P., et al., Mallory-Denk-bodies: lessons from keratin-containing hepatic inclusion bodies. Biochim Biophys Acta, 2008. 1782(12): p. 764-74.

281. Zatloukal, K., et al., From Mallory to Mallory-Denk bodies: what, how and why? Exp Cell Res, 2007. 313(10): p. 2033-49.

282. Hull, R.L., et al., Islet amyloid: a critical entity in the pathogenesis of type 2 diabetes. J Clin Endocrinol Metab, 2004. 89(8): p. 3629-43.

283. Redmann, M., et al., Mitophagy mechanisms and role in human diseases. Int $\mathrm{J}$ Biochem Cell Biol, 2014. 53: p. 127-33.

284. Chhangani, D. and A. Mishra, Protein quality control system in neurodegeneration: a healing company hard to beat but failure is fatal. Mol Neurobiol, 2013. 48(1): p. 141-56.

285. Spillantini, M.G. and M. Goedert, The alpha-synucleinopathies: Parkinson's disease, dementia with Lewy bodies, and multiple system atrophy. Ann N Y Acad Sci, 2000. 920: p. 16-27.

286. Winslow, A.R., et al., alpha-Synuclein impairs macroautophagy: implications for Parkinson's disease. J Cell Biol, 2010. 190(6): p. 1023-37. 
287. Dehay, B., et al., Pathogenic lysosomal depletion in Parkinson's disease. J Neurosci, 2010. 30(37): p. 12535-44.

288. Subramaniam, S.R., et al., Region specific mitochondrial impairment in mice with widespread overexpression of alpha-synuclein. Neurobiol Dis, 2014. 70: p. 204-13.

289. Stafa, K., et al., Functional interaction of Parkinson's disease-associated LRRK2 with members of the dynamin GTPase superfamily. Hum Mol Genet, 2014. 23(8): p. 2055-77.

290. Fan, H.C., et al., Polyglutamine (PolyQ) diseases: genetics to treatments. Cell Transplant, 2014. 23(4-5): p. 441-58.

291. Shao, J. and M.I. Diamond, Polyglutamine diseases: emerging concepts in pathogenesis and therapy. Hum Mol Genet, 2007. 16 Spec No. 2: p. R115-23.

292. Shibata, M., et al., Regulation of intracellular accumulation of mutant Huntingtin by Beclin 1. J Biol Chem, 2006. 281(20): p. 14474-85.

293. Ravikumar, B., et al., Inhibition of mTOR induces autophagy and reduces toxicity of polyglutamine expansions in fly and mouse models of Huntington disease. Nat Genet, 2004. 36(6): p. 585-95.

294. Panov, A.V., et al., Early mitochondrial calcium defects in Huntington's disease are a direct effect of polyglutamines. Nat Neurosci, 2002. 5(8): p. 731-6.

295. Cui, L., et al., Transcriptional repression of PGC-1 alpha by mutant huntingtin leads to mitochondrial dysfunction and neurodegeneration. Cell, 2006. 127(1): p. 59-69.

296. Chang, D.T., et al., Mutant huntingtin aggregates impair mitochondrial movement and trafficking in cortical neurons. Neurobiol Dis, 2006. 22(2): p. 388-400.

297. Beckman, J.S., et al., Superoxide dismutase and the death of motoneurons in ALS. Trends Neurosci, 2001. 24(11 Suppl): p. S15-20.

298. Sreedharan, J., et al., TDP-43 mutations in familial and sporadic amyotrophic lateral sclerosis. Science, 2008. 319(5870): p. 1668-72. 
299. Wang, W., et al., The ALS disease-associated mutant TDP-43 impairs mitochondrial dynamics and function in motor neurons. Hum Mol Genet, 2013. 22(23): p. 4706-19.

300. Palomo, G.M. and G. Manfredi, Exploring new pathways of neurodegeneration in ALS: the role of mitochondria quality control. Brain Res, 2015. 1607: p. 36-46.

301. Stoica, R., et al., ER-mitochondria associations are regulated by the VAPBPTPIP51 interaction and are disrupted by ALS/FTD-associated TDP-43. Nat Commun, 2014. 5: p. 3996.

302. Teyssou, E., et al., Mutations in SQSTM1 encoding p62 in amyotrophic lateral sclerosis: genetics and neuropathology. Acta Neuropathol, 2013. 125(4): p. 51122.

303. O'Connor, D.M. and N.M. Boulis, Gene therapy for neurodegenerative diseases. Trends Mol Med, 2015. 21(8): p. 504-12.

304. Sarkar, S. and D.C. Rubinsztein, Small molecule enhancers of autophagy for neurodegenerative diseases. Mol Biosyst, 2008. 4(9): p. 895-901.

305. Rubinsztein, D.C., C.F. Bento, and V. Deretic, Therapeutic targeting of autophagy in neurodegenerative and infectious diseases. J Exp Med, 2015. 212(7): p. 97990.

306. Harris, H. and D.C. Rubinsztein, Control of autophagy as a therapy for neurodegenerative disease. Nat Rev Neurol, 2012. 8(2): p. 108-17.

307. Rubinsztein, D.C., P. Codogno, and B. Levine, Autophagy modulation as a potential therapeutic target for diverse diseases. Nat Rev Drug Discov, 2012. 11(9): p. 709-30.

308. Jahrling, J.B. and R.M. Laberge, Age-Related Neurodegeneration Prevention Through mTOR Inhibition: Potential Mechanisms and Remaining Questions. Curr Top Med Chem, 2015. 15(21): p. 2139-51. 
309. Caccamo, A., et al., Molecular interplay between mammalian target of rapamycin (mTOR), amyloid-beta, and Tau: effects on cognitive impairments. J Biol Chem, 2010. 285(17): p. 13107-20.

310. Spilman, P., et al., Inhibition of mTOR by rapamycin abolishes cognitive deficits and reduces amyloid-beta levels in a mouse model of Alzheimer's disease. PLoS One, 2010. 5(4): p. e9979.

311. Berger, Z., et al., Rapamycin alleviates toxicity of different aggregate-prone proteins. Hum Mol Genet, 2006. 15(3): p. 433-42.

312. Menzies, F.M., et al., Autophagy induction reduces mutant ataxin-3 levels and toxicity in a mouse model of spinocerebellar ataxia type 3. Brain, 2010. 133(Pt 1): p. 93-104.

313. Cortes, C.J., et al., Rapamycin delays disease onset and prevents PrP plaque deposition in a mouse model of Gerstmann-Straussler-Scheinker disease. J Neurosci, 2012. 32(36): p. 12396-405.

314. Wang, I.F., et al., Autophagy activators rescue and alleviate pathogenesis of a mouse model with proteinopathies of the TAR DNA-binding protein 43. Proc Natl Acad Sci U S A, 2012. 109(37): p. 15024-9.

315. Sarbassov, D.D., S.M. Ali, and D.M. Sabatini, Growing roles for the mTOR pathway. Curr Opin Cell Biol, 2005. 17(6): p. 596-603.

316. Ma, T.C., et al., Metformin therapy in a transgenic mouse model of Huntington's disease. Neurosci Lett, 2007. 411(2): p. 98-103.

317. Kickstein, E., et al., Biguanide metformin acts on tau phosphorylation via mTOR/protein phosphatase 2A (PP2A) signaling. Proc Natl Acad Sci U S A, 2010. 107(50): p. 21830-5.

318. Salminen, A., et al., AMP-activated protein kinase: a potential player in Alzheimer's disease. J Neurochem, 2011. 118(4): p. 460-74. 
319. Kim, J., et al., AMPK activation inhibits apoptosis and tau hyperphosphorylation mediated by palmitate in SH-SY5Y cells. Brain Res, 2011. 1418: p. 42-51.

320. Sarkar, S., et al., A rational mechanism for combination treatment of Huntington's disease using lithium and rapamycin. Hum Mol Genet, 2008. 17(2): p. 170-8.

321. Nakaso, K., et al., Novel cytoprotective mechanism of anti-parkinsonian drug deprenyl: PI3K and Nrf2-derived induction of antioxidative proteins. Biochem Biophys Res Commun, 2006. 339(3): p. 915-22.

322. Li, G., et al., HDAC6 alpha-tubulin deacetylase: a potential therapeutic target in neurodegenerative diseases. J Neurol Sci, 2011. 304(1-2): p. 1-8.

323. Dallavalle, S., C. Pisano, and F. Zunino, Development and therapeutic impact of HDAC6-selective inhibitors. Biochem Pharmacol, 2012. 84(6): p. 756-65.

324. Dompierre, J.P., et al., Histone deacetylase 6 inhibition compensates for the transport deficit in Huntington's disease by increasing tubulin acetylation. J Neurosci, 2007. 27(13): p. 3571-83.

325. Kozikowski, A.P., et al., Functional differences in epigenetic modulators-superiority of mercaptoacetamide-based histone deacetylase inhibitors relative to hydroxamates in cortical neuron neuroprotection studies. J Med Chem, 2007. 50(13): p. 3054-61.

326. Ding, H., P.J. Dolan, and G.V. Johnson, Histone deacetylase 6 interacts with the microtubule-associated protein tau. J Neurochem, 2008. 106(5): p. 2119-30.

327. Kilgore, M., et al., Inhibitors of class 1 histone deacetylases reverse contextual memory deficits in a mouse model of Alzheimer's disease. Neuropsychopharmacology, 2010. 35(4): p. 870-80.

328. Vives-Bauza, C., et al., PINK1-dependent recruitment of Parkin to mitochondria in mitophagy. Proc Natl Acad Sci U S A, 2010. 107(1): p. 378-83. 
329. East, D.A., et al., PMI: a DeltaPsim independent pharmacological regulator of mitophagy. Chem Biol, 2014. 21(11): p. 1585-96.

330. Colman, R.J., et al., Caloric restriction delays disease onset and mortality in rhesus monkeys. Science, 2009. 325(5937): p. 201-4.

331. Greer, E.L., et al., An AMPK-FOXO pathway mediates longevity induced by a novel method of dietary restriction in C. elegans. Curr Biol, 2007. 17(19): p. 164656.

332. Morselli, E., et al., Caloric restriction and resveratrol promote longevity through the Sirtuin-1-dependent induction of autophagy. Cell Death Dis, 2010. 1: p. e10.

333. Schriner, S.E., et al., Extension of Drosophila Lifespan by Rhodiola rosea Depends on Dietary Carbohydrate and Caloric Content in a Simplified Diet. J Med Food, 2016. 19(3): p. 318-23.

334. Schriner, S.E., et al., Extension of Drosophila lifespan by Rhodiola rosea through a mechanism independent from dietary restriction. PLoS One, 2013. 8(5): p. e63886.

335. Simonsen, A., et al., Promoting basal levels of autophagy in the nervous system enhances longevity and oxidant resistance in adult Drosophila. Autophagy, 2008. 4(2): p. 176-84.

336. Hars, E.S., et al., Autophagy regulates ageing in C. elegans. Autophagy, 2007. 3(2): p. 93-5.

337. Sengupta, A., J.D. Molkentin, and K.E. Yutzey, FoxO transcription factors promote autophagy in cardiomyocytes. J Biol Chem, 2009. 284(41): p. 28319-31.

338. Brunet, A., et al., Stress-dependent regulation of FOXO transcription factors by the SIRT1 deacetylase. Science, 2004. 303(5666): p. 2011-5.

339. Cuervo, A.M., et al., Autophagy and aging: the importance of maintaining "clean" cells. Autophagy, 2005. 1(3): p. 131-40. 
340. Sohal, R.S. and R. Weindruch, Oxidative stress, caloric restriction, and aging. Science, 1996. 273(5271): p. 59-63.

341. Esposito, E., et al., A review of specific dietary antioxidants and the effects on biochemical mechanisms related to neurodegenerative processes. Neurobiol Aging, 2002. 23(5): p. 719-35.

342. Harrison, D.E., et al., Rapamycin fed late in life extends lifespan in genetically heterogeneous mice. Nature, 2009. 460(7253): p. 392-5.

343. Williamson, D.A., et al., Is caloric restriction associated with development of eatingdisorder symptoms? Results from the CALERIE trial. Health Psychol, 2008. 27(1s): p. S32-42.

344. Ramos, S., Effects of dietary flavonoids on apoptotic pathways related to cancer chemoprevention. J Nutr Biochem, 2007. 18(7): p. 427-42.

345. Urquiaga, I. and F. Leighton, Plant polyphenol antioxidants and oxidative stress. Biol Res, 2000. 33(2): p. 55-64.

346. Ebrahimi, A. and H. Schluesener, Natural polyphenols against neurodegenerative disorders: potentials and pitfalls. Ageing Res Rev, 2012. 11(2): p. 329-45.

347. Burns, J., et al., Plant foods and herbal sources of resveratrol. J Agric Food Chem, 2002. 50(11): p. 3337-40.

348. Vingtdeux, V., et al., AMP-activated protein kinase signaling activation by resveratrol modulates amyloid-beta peptide metabolism. J Biol Chem, 2010. 285(12): p. 9100-13.

349. Karuppagounder, S.S., et al., Dietary supplementation with resveratrol reduces plaque pathology in a transgenic model of Alzheimer's disease. Neurochem Int, 2009. 54(2): p. 111-8. 
350. Lagouge, M., et al., Resveratrol improves mitochondrial function and protects against metabolic disease by activating SIRT1 and PGC-1alpha. Cell, 2006. 127(6): p. 1109-22.

351. Wang, B., et al., Resveratrol preserves mitochondrial function, stimulates mitochondrial biogenesis, and attenuates oxidative stress in regulatory $T$ cells of mice fed a high-fat diet. J Food Sci, 2014. 79(9): p. H1823-31.

352. Waltner-Law, M.E., et al., Epigallocatechin gallate, a constituent of green tea, represses hepatic glucose production. J Biol Chem, 2002. 277(38): p. 34933-40.

353. Dashwood, W.M., et al., Lysosomal trafficking of beta-catenin induced by the tea polyphenol epigallocatechin-3-gallate. Mutat Res, 2005. 591(1-2): p. 161-72.

354. Devika, P.T. and P.S. Prince, Preventive effect of (-)epigallocatechin-gallate (EGCG) on lysosomal enzymes in heart and subcellular fractions in isoproterenolinduced myocardial infarcted Wistar rats. Chem Biol Interact, 2008. 172(3): p. 24552.

355. Leone, M., et al., Cancer prevention by tea polyphenols is linked to their direct inhibition of antiapoptotic Bcl-2-family proteins. Cancer Res, 2003. 63(23): p. 811821.

356. Ehrnhoefer, D.E., et al., Green tea (-)-epigallocatechin-gallate modulates early events in huntingtin misfolding and reduces toxicity in Huntington's disease models. Hum Mol Genet, 2006. 15(18): p. 2743-51.

357. Ehrnhoefer, D.E., et al., EGCG redirects amyloidogenic polypeptides into unstructured, off-pathway oligomers. Nat Struct Mol Biol, 2008. 15(6): p. 558-66.

358. Mandel, S., G. Maor, and M.B. Youdim, Iron and alpha-synuclein in the substantia nigra of MPTP-treated mice: effect of neuroprotective drugs $R$-apomorphine and green tea polyphenol (-)-epigallocatechin-3-gallate. J Mol Neurosci, 2004. 24(3): p. 401-16.

359. Singh, S., From exotic spice to modern drug? Cell, 2007. 130(5): p. 765-8. 
360. Hafner-Bratkovic, l., et al., Curcumin binds to the alpha-helical intermediate and to the amyloid form of prion protein - a new mechanism for the inhibition of $\operatorname{PrP}(S c)$ accumulation. J Neurochem, 2008. 104(6): p. 1553-64.

361. Wang, Y.J., et al., Consumption of grape seed extract prevents amyloid-beta deposition and attenuates inflammation in brain of an Alzheimer's disease mouse. Neurotox Res, 2009. 15(1): p. 3-14.

362. Jurenka, J.S., Anti-inflammatory properties of curcumin, a major constituent of Curcuma longa: a review of preclinical and clinical research. Altern Med Rev, 2009. 14(2): p. 141-53.

363. Reuter, S., et al., Modulation of anti-apoptotic and survival pathways by curcumin as a strategy to induce apoptosis in cancer cells. Biochem Pharmacol, 2008. 76(11): p. 1340-51.

364. Kuo, J.J., et al., Curcumin ameliorates mitochondrial dysfunction associated with inhibition of gluconeogenesis in free fatty acid-mediated hepatic lipoapoptosis. Int J Mol Med, 2012. 30(3): p. 643-9.

365. Ahmed, A.H., et al., Pomegranate extract modulates processing of amyloid-beta precursor protein in an aged Alzheimer's disease animal model. Curr Alzheimer Res, 2014. 11(9): p. 834-43.

366. Hartman, R.E., et al., Pomegranate juice decreases amyloid load and improves behavior in a mouse model of Alzheimer's disease. Neurobiol Dis, 2006. 24(3): p. 506-15.

367. Ryu, D., et al., Urolithin A induces mitophagy and prolongs lifespan in C. elegans and increases muscle function in rodents. Nat Med, 2016. 22(8): p. 879-88.

368. Nair, V., et al., Pomegranate extract induces cell cycle arrest and alters cellular phenotype of human pancreatic cancer cells. Anticancer Res, 2011. 31(9): p. 2699-704. 
369. Dai, Z., et al., Pomegranate extract inhibits the proliferation and viability of MMTVWnt-1 mouse mammary cancer stem cells in vitro. Oncol Rep, 2010. 24(4): p. 1087-91.

370. Sridharan, S., K. Jain, and A. Basu, Regulation of autophagy by kinases. Cancers (Basel), 2011. 3(2): p. 2630-54.

371. McEwan, D.G. and I. Dikic, The Three Musketeers of Autophagy: phosphorylation, ubiquitylation and acetylation. Trends Cell Biol, 2011. 21(4): p. 195-201.

372. Faria, A. and C. Calhau, The bioactivity of pomegranate: impact on health and disease. Crit Rev Food Sci Nutr, 2011. 51(7): p. 626-34.

373. Zarfeshany, A., S. Asgary, and S.H. Javanmard, Potent health effects of pomegranate. Adv Biomed Res, 2014. 3: p. 100.

374. Kimura, S., T. Noda, and T. Yoshimori, Dissection of the autophagosome maturation process by a novel reporter protein, tandem fluorescent-tagged LC3. Autophagy, 2007. 3(5): p. 452-60.

375. de Castro, E., et al., ScanProsite: detection of PROSITE signature matches and ProRule-associated functional and structural residues in proteins. Nucleic Acids Res, 2006. 34(Web Server issue): p. W362-5.

376. Obenauer, J.C., L.C. Cantley, and M.B. Yaffe, Scansite 2.0: Proteome-wide prediction of cell signaling interactions using short sequence motifs. Nucleic Acids Res, 2003. 31(13): p. 3635-41.

377. Kinoshita, E., E. Kinoshita-Kikuta, and T. Koike, Separation and detection of large phosphoproteins using Phos-tag SDS-PAGE. Nat Protoc, 2009. 4(10): p. 1513-21.

378. Frezza, C., S. Cipolat, and L. Scorrano, Organelle isolation: functional mitochondria from mouse liver, muscle and cultured fibroblasts. Nat Protoc, 2007. 2(2): p. 287-95. 
379. Wieckowski, M.R. and L. Wojtczak, Isolation of crude mitochondrial fraction from cells. Methods Mol Biol, 2015. 1241: p. 1-8.

380. Ahmad, T., et al., Computational classification of mitochondrial shapes reflects stress and redox state. Cell Death Dis, 2013. 4: p. e461.

381. Hernandez, G., et al., MitoTimer: a novel tool for monitoring mitochondrial turnover. Autophagy, 2013. 9(11): p. 1852-61.

382. Ferree, A.W., et al., Mito Timer probe reveals the impact of autophagy, fusion, and motility on subcellular distribution of young and old mitochondrial protein and on relative mitochondrial protein age. Autophagy, 2013. 9(11): p. 1887-96.

383. Laker, R.C., et al., A novel MitoTimer reporter gene for mitochondrial content, structure, stress, and damage in vivo. J Biol Chem, 2014. 289(17): p. 12005-15.

384. Kruger, R., The role of synphilin-1 in synaptic function and protein degradation. Cell Tissue Res, 2004. 318(1): p. 195-9.

385. Li, T., J. Liu, and W.W. Smith, Synphilin-1 binds ATP and regulates intracellular energy status. PLoS One, 2014. 9(12): p. e115233.

386. Li, X., et al., A novel obesity model: synphilin-1-induced hyperphagia and obesity in mice. Int J Obes (Lond), 2012. 36(9): p. 1215-21.

387. Liu, J., et al., Synphilin-1 alters metabolic homeostasis in a novel Drosophila obesity model. Int J Obes (Lond), 2012. 36(12): p. 1529-36.

388. Hunter, T., The age of crosstalk: phosphorylation, ubiquitination, and beyond. Mol Cell, 2007. 28(5): p. 730-8.

389. Tan, J.M., et al., Lysine 63-linked ubiquitination promotes the formation and autophagic clearance of protein inclusions associated with neurodegenerative diseases. Hum Mol Genet, 2008. 17(3): p. 431-9. 
390. Ryo, A., et al., Prolyl-isomerase Pin1 accumulates in lewy bodies of parkinson disease and facilitates formation of alpha-synuclein inclusions. J Biol Chem, 2006. 281(7): p. 4117-25.

391. Saito, N. and Y. Shirai, Protein kinase C gamma (PKC gamma): function of neuron specific isotype. J Biochem, 2002. 132(5): p. 683-7.

392. Hunter, T. and M. Karin, The regulation of transcription by phosphorylation. Cell, 1992. 70(3): p. 375-87.

393. Pickart, C.M., Mechanisms underlying ubiquitination. Annu Rev Biochem, 2001. 70: p. 503-33.

394. Berndsen, C.E. and C. Wolberger, New insights into ubiquitin E3 ligase mechanism. Nat Struct Mol Biol, 2014. 21(4): p. 301-7.

395. Kazlauskaite, A., et al., Binding to serine 65-phosphorylated ubiquitin primes Parkin for optimal PINK1-dependent phosphorylation and activation. EMBO Rep, 2015. 16(8): p. 939-54.

396. Shiba-Fukushima, K., et al., PINK1-mediated phosphorylation of the Parkin ubiquitin-like domain primes mitochondrial translocation of Parkin and regulates mitophagy. Sci Rep, 2012. 2: p. 1002.

397. Tzulker, R., et al., Antioxidant activity, polyphenol content, and related compounds in different fruit juices and homogenates prepared from 29 different pomegranate accessions. J Agric Food Chem, 2007. 55(23): p. 9559-70.

398. Sandoval-Acuna, C., J. Ferreira, and H. Speisky, Polyphenols and mitochondria: an update on their increasingly emerging ROS-scavenging independent actions. Arch Biochem Biophys, 2014. 559: p. 75-90.

399. Teixeira, J., et al., Dietary Polyphenols and Mitochondrial Function: Role in Health and Disease. Curr Med Chem, 2017. 
400. Bratic, A. and N.G. Larsson, The role of mitochondria in aging. J Clin Invest, 2013. 123(3): p. 951-7.

401. Sun, N., R.J. Youle, and T. Finkel, The Mitochondrial Basis of Aging. Mol Cell, 2016. 61(5): p. 654-66.

402. Johanningsmeier, S.D. and G.K. Harris, Pomegranate as a functional food and nutraceutical source. Annu Rev Food Sci Technol, 2011. 2: p. 181-201.

403. Panth, N., B. Manandhar, and K.R. Paudel, Anticancer Activity of Punica granatum (Pomegranate): A Review. Phytother Res, 2017. 31(4): p. 568-578.

404. Turrini, E., L. Ferruzzi, and C. Fimognari, Potential Effects of Pomegranate Polyphenols in Cancer Prevention and Therapy. Oxid Med Cell Longev, 2015. 2015: p. 938475.

405. Viladomiu, M., et al., Preventive and prophylactic mechanisms of action of pomegranate bioactive constituents. Evid Based Complement Alternat Med, 2013. 2013: p. 789764.

406. Balasubramani, S.P., et al., Pomegranate Juice Enhances Healthy Lifespan in Drosophila melanogaster: An Exploratory Study. Front Public Health, 2014. 2: p. 245.

407. Kilicgun, H., N. Arda, and E.O. Ucar, Identification of longevity, fertility and growthpromoting properties of pomegranate in Caenorhabditis elegans. Pharmacogn Mag, 2015. 11(42): p. 356-9.

408. Ryter, S.W., S.M. Cloonan, and A.M. Choi, Autophagy: a critical regulator of cellular metabolism and homeostasis. Mol Cells, 2013. 36(1): p. 7-16.

409. Cuervo, A.M., Autophagy in neurons: it is not all about food. Trends in molecular medicine, 2006. 12(10): p. 461-464.

410. Wang, S.G., et al., Punicalagin induces apoptotic and autophagic cell death in human U87MG glioma cells. Acta Pharmacol Sin, 2013. 34(11): p. 1411-9. 
411. Chen, B., et al., Pomegranate juice and punicalagin attenuate oxidative stress and apoptosis in human placenta and in human placental trophoblasts. Am J Physiol Endocrinol Metab, 2012. 302(9): p. E1142-52.

412. Feng, R., et al., Delphinidin induces necrosis in hepatocellular carcinoma cells in the presence of 3-methyladenine, an autophagy inhibitor. J Agric Food Chem, 2010. 58(7): p. 3957-64.

413. Yla-Anttila, P., et al., Monitoring autophagy by electron microscopy in Mammalian cells. Methods Enzymol, 2009. 452: p. 143-64.

414. Lystad, A.H. and A. Simonsen, Assays to monitor aggrephagy. Methods, 2015. 75: p. 112-9.

415. Tan, S. and E. Wong, Kinetics of Protein Aggregates Disposal by Aggrephagy. Methods Enzymol, 2017. 588: p. 245-281.

416. Lieberman, A.P., et al., Autophagy in lysosomal storage disorders. Autophagy, 2012. 8(5): p. 719-30.

417. Roskoski, R., Jr., ERK1/2 MAP kinases: structure, function, and regulation. Pharmacol Res, 2012. 66(2): p. 105-43.

418. Nave, B.T., et al., Mammalian target of rapamycin is a direct target for protein kinase B: identification of a convergence point for opposing effects of insulin and amino-acid deficiency on protein translation. Biochem J, 1999. 344 Pt 2: p. 42731.

419. Berlett, B.S. and E.R. Stadtman, Protein oxidation in aging, disease, and oxidative stress. J Biol Chem, 1997. 272(33): p. 20313-6.

420. Sohal, R.S. and A. Dubey, Mitochondrial oxidative damage, hydrogen peroxide release, and aging. Free Radic Biol Med, 1994. 16(5): p. 621-6. 
421. Gil, M.l., et al., Antioxidant activity of pomegranate juice and its relationship with phenolic composition and processing. J Agric Food Chem, 2000. 48(10): p. 4581 9.

422. Palikaras, K., E. Lionaki, and N. Tavernarakis, Mitophagy: In sickness and in health. Mol Cell Oncol, 2016. 3(1): p. e1056332.

423. Zhang, X., et al., MCOLN1 is a ROS sensor in lysosomes that regulates autophagy. Nat Commun, 2016. 7: p. 12109.

424. Zhang, X., L. Yu, and H. Xu, Lysosome calcium in ROS regulation of autophagy. Autophagy, 2016. 12(10): p. 1954-1955.

425. Ivankovic, D., et al., Mitochondrial and lysosomal biogenesis are activated following PINK1/parkin-mediated mitophagy. J Neurochem, 2016. 136(2): p. 388402.

426. Liu, X. and G. Hajnoczky, Altered fusion dynamics underlie unique morphological changes in mitochondria during hypoxia-reoxygenation stress. Cell Death Differ, 2011. 18(10): p. 1561-72.

427. Narendra, D., et al., Parkin is recruited selectively to impaired mitochondria and promotes their autophagy. J Cell Biol, 2008. 183(5): p. 795-803.

428. Matsuda, N., et al., PINK1 stabilized by mitochondrial depolarization recruits Parkin to damaged mitochondria and activates latent Parkin for mitophagy. J Cell Biol, 2010. 189(2): p. 211-21.

429. Narendra, D.P., et al., PINK1 is selectively stabilized on impaired mitochondria to activate Parkin. PLoS Biol, 2010. 8(1): p. e1000298.

430. Siddiqui, A., et al., Mitochondrial Quality Control via the PGC1alpha-TFEB Signaling Pathway Is Compromised by Parkin Q311X Mutation But Independently Restored by Rapamycin. J Neurosci, 2015. 35(37): p. 12833-44. 
431. Seo, A.Y., et al., New insights into the role of mitochondria in aging: mitochondrial dynamics and more. J Cell Sci, 2010. 123(Pt 15): p. 2533-42.

432. Clementi, M.E., et al., Punicalagin reduces H2O2-induced cytotoxicity and apoptosis in PC12 cells by modulating the levels of reactive oxygen species. Nutr Neurosci, 2017: p. 1-8.

433. Hollebeeck, S., et al., Anti-inflammatory effects of pomegranate (Punica granatum L.) husk ellagitannins in Caco-2 cells, an in vitro model of human intestine. Food Funct, 2012. 3(8): p. 875-85.

434. Seeram, N.P., et al., In vitro antiproliferative, apoptotic and antioxidant activities of punicalagin, ellagic acid and a total pomegranate tannin extract are enhanced in combination with other polyphenols as found in pomegranate juice. J Nutr Biochem, 2005. 16(6): p. 360-7.

435. Wang, Y., et al., Punicalagin promotes autophagy to protect primary human syncytiotrophoblasts from apoptosis. Reproduction, 2016. 151(2): p. 97-104.

436. Zahin, M., et al., Punicalagin and ellagic acid demonstrate antimutagenic activity and inhibition of benzo[a]pyrene induced DNA adducts. Biomed Res Int, 2014. 2014: p. 467465.

437. Chen, P., et al., Luteolin inhibits cell proliferation and induces cell apoptosis via down-regulation of mitochondrial membrane potential in esophageal carcinoma cells EC1 and KYSE450. Oncotarget, 2017. 8(16): p. 27471-27480.

438. Ma, L., et al., Luteolin exerts an anticancer effect on NCI-H460 human non-small cell lung cancer cells through the induction of Sirt1-mediated apoptosis. Mol Med Rep, 2015. 12(3): p. 4196-202.

439. Yun, J.M., et al., Delphinidin, an anthocyanidin in pigmented fruits and vegetables, induces apoptosis and cell cycle arrest in human colon cancer HCT116 cells. Mol Carcinog, 2009. 48(3): p. 260-70. 
440. Pal, H.C., et al., Delphinidin reduces cell proliferation and induces apoptosis of non-small-cell lung cancer cells by targeting EGFR/VEGFR2 signaling pathways. PLoS One, 2013. 8(10): p. e77270.

441. Tooze, S.A. and I. Dikic, Autophagy Captures the Nobel Prize. Cell, 2016. 167(6): p. 1433-1435.

442. Mellor, H. and P.J. Parker, The extended protein kinase $C$ superfamily. Biochem J, 1998. 332 ( Pt 2): p. 281-92.

443. Takayama, K., A. Matsuura, and E. Itakura, Dissection of ubiquitinated protein degradation by basal autophagy. FEBS Lett, 2017. 591(9): p. 1199-1211.

444. Oh, H.G., et al., Regulation of basal autophagy by transient receptor potential melastatin 7 (TRPM7) channel. Biochem Biophys Res Commun, 2015. 463(1-2): p. 7-12.

445. Pinna, L.A., Casein kinase 2: an 'eminence grise' in cellular regulation? Biochim Biophys Acta, 1990. 1054(3): p. 267-84.

446. Ryu, M.Y., et al., Localization of CKIl beta subunits in Lewy bodies of Parkinson's disease. J Neurol Sci, 2008. 266(1-2): p. 9-12.

447. Fiesel, F.C., et al., A specific subset of E2 ubiquitin-conjugating enzymes regulate Parkin activation and mitophagy differently. J Cell Sci, 2014. 127(Pt 16): p. 3488504.

448. Doss-Pepe, E.W., L. Chen, and K. Madura, Alpha-synuclein and parkin contribute to the assembly of ubiquitin lysine 63-linked multiubiquitin chains. J Biol Chem, 2005. 280(17): p. 16619-24.

449. Zhang, Y., et al., Parkin functions as an E2-dependent ubiquitin- protein ligase and promotes the degradation of the synaptic vesicle-associated protein, CDCrel-1. Proc Natl Acad Sci U S A, 2000. 97(24): p. 13354-9. 
450. Kroemer, G., G. Marino, and B. Levine, Autophagy and the integrated stress response. Mol Cell, 2010. 40(2): p. 280-93.

451. Braidy, N., et al., Consumption of pomegranates improves synaptic function in a transgenic mice model of Alzheimer's disease. Oncotarget, 2016. 7(40): p. 6458964604.

452. Hasima, N. and B. Ozpolat, Regulation of autophagy by polyphenolic compounds as a potential therapeutic strategy for cancer. Cell Death Dis, 2014. 5: p. e1509.

453. Yuan, T., et al., Pomegranate's Neuroprotective Effects against Alzheimer's Disease Are Mediated by Urolithins, Its Ellagitannin-Gut Microbial Derived Metabolites. ACS Chem Neurosci, 2016. 7(1): p. 26-33.

454. Putics, A., et al., Resveratrol induces the heat-shock response and protects human cells from severe heat stress. Antioxid Redox Signal, 2008. 10(1): p. 65-75.

455. Kuzuhara, T., M. Suganuma, and H. Fujiki, Green tea catechin as a chemical chaperone in cancer prevention. Cancer Lett, 2008. 261(1): p. 12-20.

456. Calabrese, E.J., Hormesis: a fundamental concept in biology. Microb Cell, 2014. 1(5): p. 145-149.

457. Fivenson, E.M., et al., Mitophagy in neurodegeneration and aging. Neurochem Int, 2017.

458. Palikaras, K., E. Lionaki, and N. Tavernarakis, Coordination of mitophagy and mitochondrial biogenesis during ageing in C. elegans. Nature, 2015. 521(7553): p. $525-8$.

459. Palikaras, K., E. Lionaki, and N. Tavernarakis, Coupling mitogenesis and mitophagy for longevity. Autophagy, 2015. 11(8): p. 1428-30.

460. Onyango, I.G., S.M. Khan, and J.P. Bennett, Jr., Mitochondria in the pathophysiology of Alzheimer's and Parkinson's diseases. Front Biosci (Landmark Ed), 2017. 22: p. 854-872. 
461. Fivenson, E.M., et al., Mitophagy in neurodegeneration and aging. Neurochem Int, 2017.

462. Sardiello, M., Transcription factor EB: from master coordinator of lysosomal pathways to candidate therapeutic target in degenerative storage diseases. Ann $\mathrm{N}$ Y Acad Sci, 2016. 1371(1): p. 3-14. 
Appendix 1: ScanProsite in-silico analysis page 1

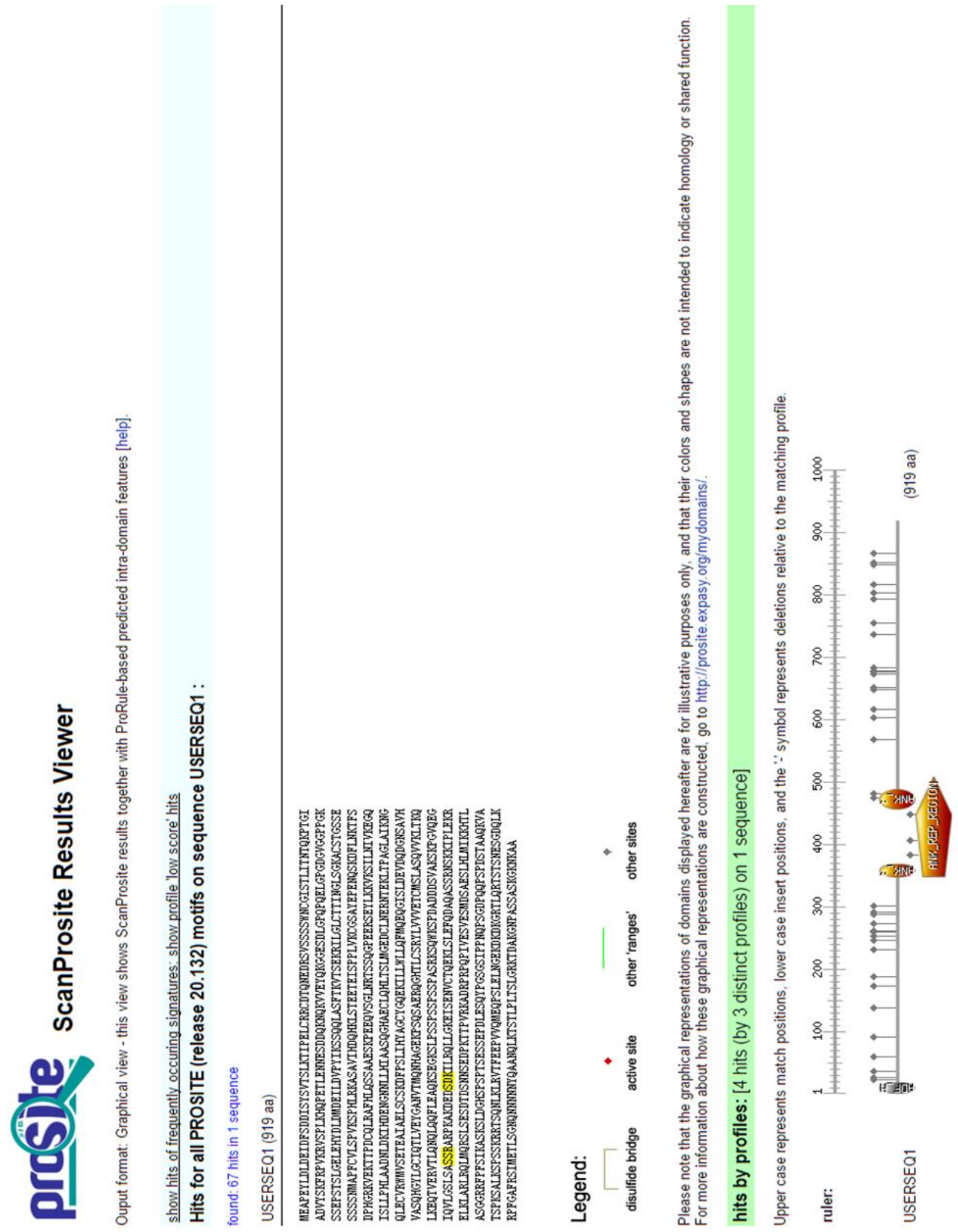


Appendix 1: ScanProsite in-silico analysis page 2

PS51263 ADF_H ADF-Hdomain profile:

1. 19: $\quad$ score $=4.847$ [waming: hit with a low confidence level $(-1)$ ]

-IMEPEY

LDLDETDFSDDIS

Predicted feature:

$\begin{array}{lllll}\text { DOMAIN } 1 & 19 & \text { ADF-H } & \text { [condition: none] }\end{array}$

\section{PS50088 ANK_REPEAT Ankynin repeat profile :}

349 - 369: $\quad$ score $=7.748$ [waming: hit with a low confidence level $(-1)$ ]

MGMLILALQGHAECLRKL----------

456 - 488: $\quad$ score $=12.155$

DGISAVIVASQHGILOCIQTLVEYGANVTIOQWH

\section{PS50297 ANK_REP_REGION Ankyrin repeat region circular profile :}

349 $-510: \quad$ score $=24.559$

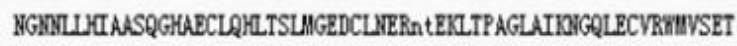

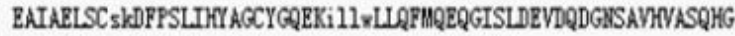

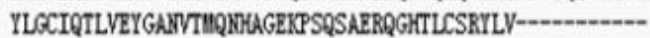

hits by profiles with a high probability of occurrence: [2 hits (by 2 distinct profiles) on 1 sequence]

Upper case represents match positions, lower case insert positions, and the "' symbol represents deletions relative to the matching profile.

ruler:

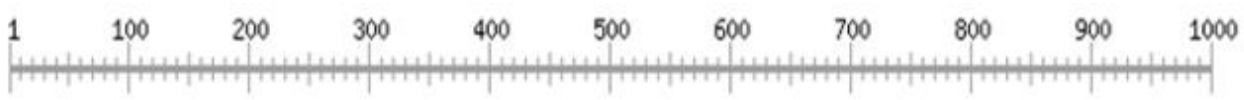

USERSEQ1

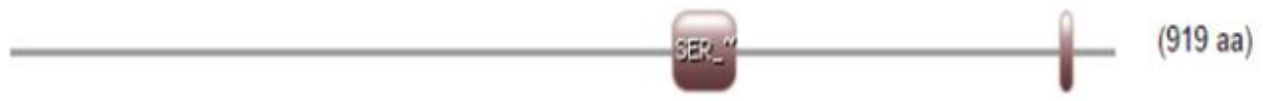

PS50324 SER_RICH Serine-rich region profile:

552 - 605: $\quad$ score $=6.625$ [warning: hit with a low confidence level $(-1)]$

Segkslpsspsspsspasrks gqkspdadddsvakskpgrqegi qvi gslsasS

PS50321 ASN_RICH Asparagine-rich region profile:

$874-885: \quad$ score $=6.536$ [waming: hit with a low confidence level $(-1)$ ]

Neqnannayqaal 


\section{Appendix 1: ScanProsite in-silico analysis page 3}

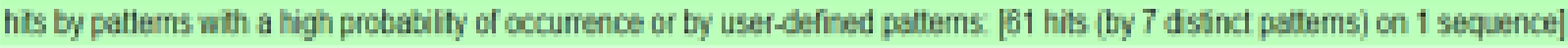

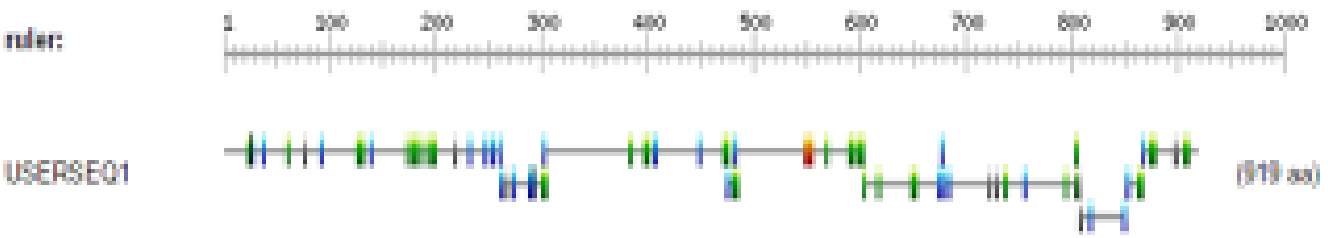

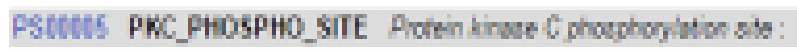

\begin{tabular}{|c|c|c|c|}
\hline \multicolumn{4}{|c|}{ Presicted hate } \\
\hline MOD_RLS & 14 & Phephesing & [condlan S] \\
\hline \multicolumn{4}{|c|}{ 60-12: In } \\
\hline \multicolumn{4}{|c|}{ Pedicis twone } \\
\hline MOD FLS & 51 & Fhophitusuln & [conditan T] \\
\hline \multicolumn{4}{|c|}{ T3-Tis } \\
\hline \multicolumn{4}{|c|}{ Pieticsol then } \\
\hline MoO FEs & 173 & Phophasins & [condlon s] \\
\hline \multicolumn{4}{|c|}{$139-191: \quad 3 d$} \\
\hline \multicolumn{4}{|c|}{ Pielicks thone } \\
\hline MOD_FES & 19 & Fhaphasind & [condthe s] \\
\hline \multicolumn{4}{|c|}{$33=35: \quad$ It } \\
\hline \multicolumn{4}{|c|}{ Preicts twhe } \\
\hline MOD_pes & M & Phosphthenine & [condibn T] \\
\hline \multicolumn{4}{|c|}{ y9-5it $\quad$ in } \\
\hline \multicolumn{4}{|c|}{ Pielicts twher } \\
\hline MO_fES & Hit & Phosphesins & [condibn S] \\
\hline $504-505$ & is & & \\
\hline \multicolumn{4}{|c|}{ Pielics twon } \\
\hline MO_REs & 44 & Phephestrs & [condthe s] \\
\hline 515-513: & in & & \\
\hline \multicolumn{4}{|c|}{ Presicted thure } \\
\hline MOD_FFs & Ets & 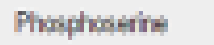 & [cothilith 3] \\
\hline
\end{tabular}


Appendix 1: ScanProsite in-silico analysis page 4

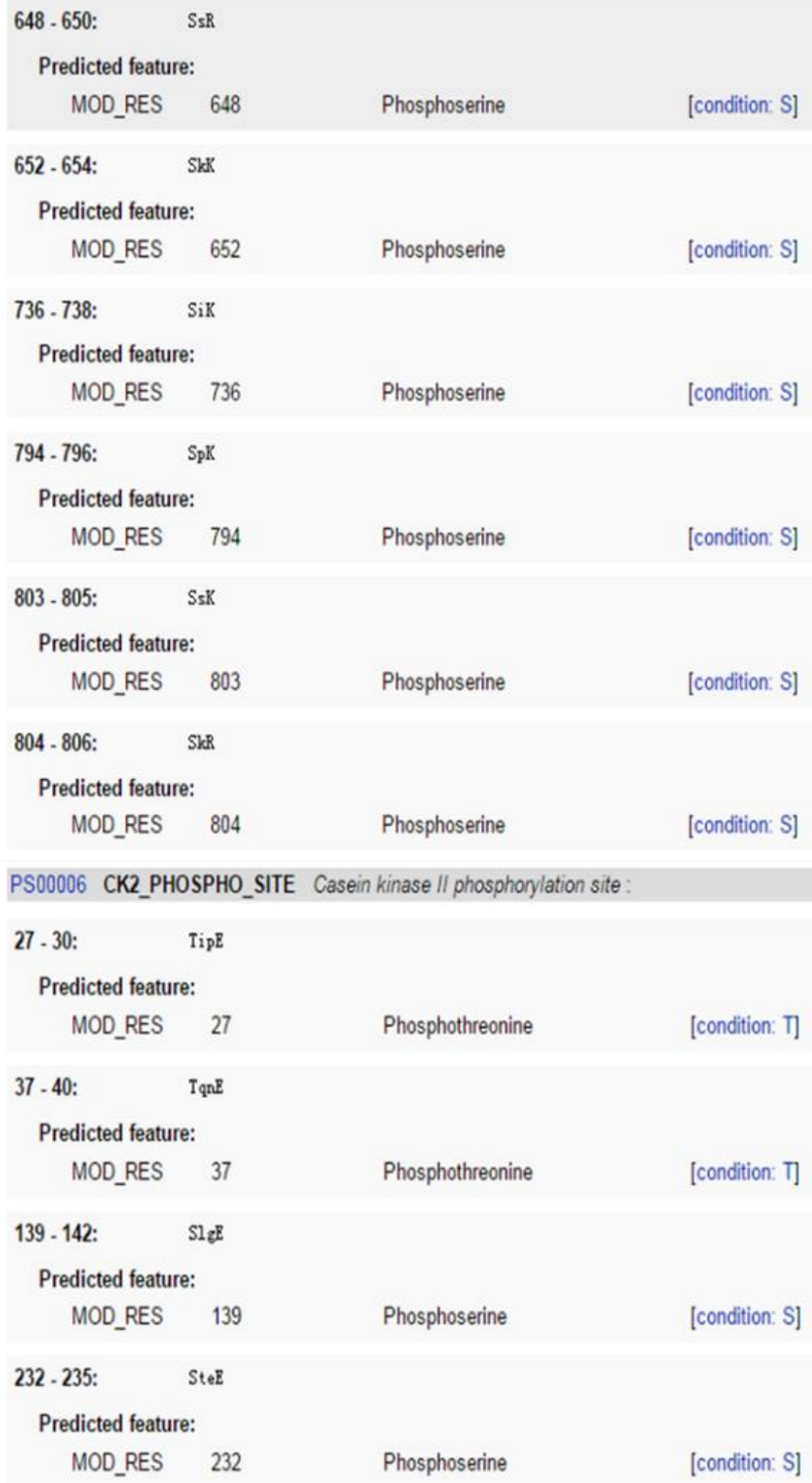


Appendix 1: ScanProsite in-silico analysis page 5

\begin{tabular}{|c|c|c|c|}
\hline $247-250:$ & Say民 & & \\
\hline \multicolumn{4}{|c|}{ Predicted feature: } \\
\hline MOD_RES & 247 & Phosphoserine & [condition: S] \\
\hline $262-265:$ & If $\mathrm{s} D$ & & \\
\hline \multicolumn{4}{|c|}{ Predicted feature: } \\
\hline MOD_RES & 262 & Phosphothreonine & [condition: T] \\
\hline $274-277:$ & ItpD & & \\
\hline \multicolumn{4}{|c|}{ Predicted feature: } \\
\hline MOD_RES & 274 & Phosphothreonine & [condition: T] \\
\hline 288 - 291: & SaaZ & & \\
\hline \multicolumn{4}{|c|}{ Predicted feature: } \\
\hline MOD_RES & 288 & Phosphoserine & [condition: S] \\
\hline $292-295:$ & Skpl & & \\
\hline \multicolumn{4}{|c|}{ Predicted feature: } \\
\hline MOD_RES & 292 & Phosphoserine & [condition: S] \\
\hline 406 - 409: & SetE & & \\
\hline \multicolumn{4}{|c|}{ Predicted feature: } \\
\hline MOD_RES & 406 & Phosphoserine & [condition: S] \\
\hline 449 - $452:$ & SIdE & & \\
\hline \multicolumn{4}{|c|}{ Predicted feature: } \\
\hline MOD_RES & 449 & Phosphoserine & [condition: S] \\
\hline 475 - 478: & Tlv? & & \\
\hline \multicolumn{4}{|c|}{ Predicted feature: } \\
\hline MOD_RES & 475 & Phosphothreonine & [condition: T] \\
\hline $674-677:$ & SlsE & & \\
\hline \multicolumn{4}{|c|}{ Predicted feature: } \\
\hline MOD_RES & 674 & Phosphoserine & [condition: S] \\
\hline $676-679:$ & SesD & & \\
\hline \multicolumn{4}{|c|}{ Predicted feature: } \\
\hline MOD_RES & 676 & Phosphoserine & [condition: S] \\
\hline
\end{tabular}


Appendix 1: ScanProsite in-silico analysis page 6

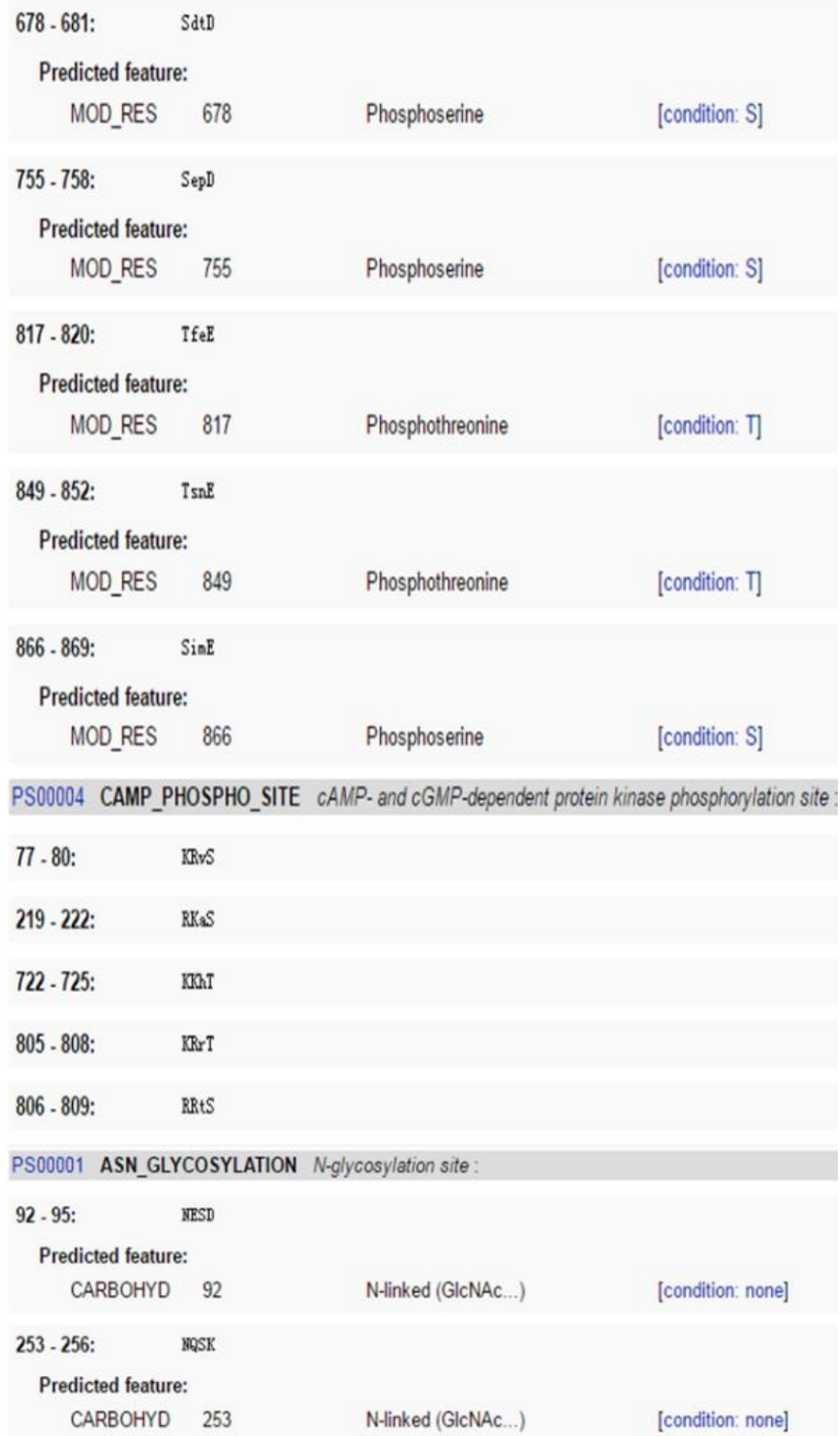


Appendix 1: ScanProsite in-silico analysis page 7

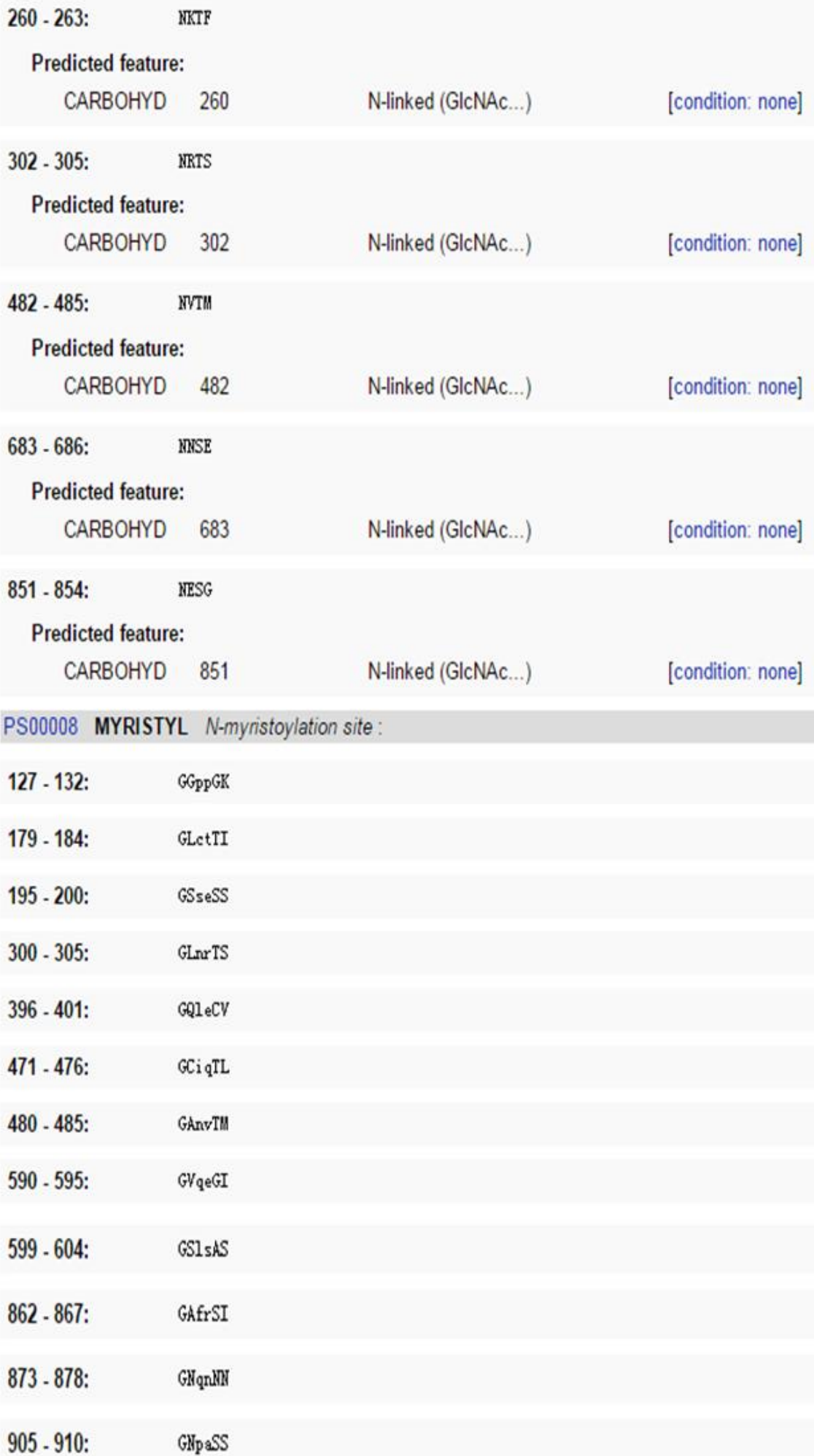


Appendix 1: ScanProsite in-silico analysis page 8

PSO0009 AMIDATION Amidation site:
267 - 270: $\quad$ bGRK
729 - 732: $\quad$ GGRR
897 - 900: $\quad$ IGRK
PS00017 ATP_GTP_A ATP/GTP-binding site motif A (P-loop):
549 - 556: $\quad$ AgkseGKS




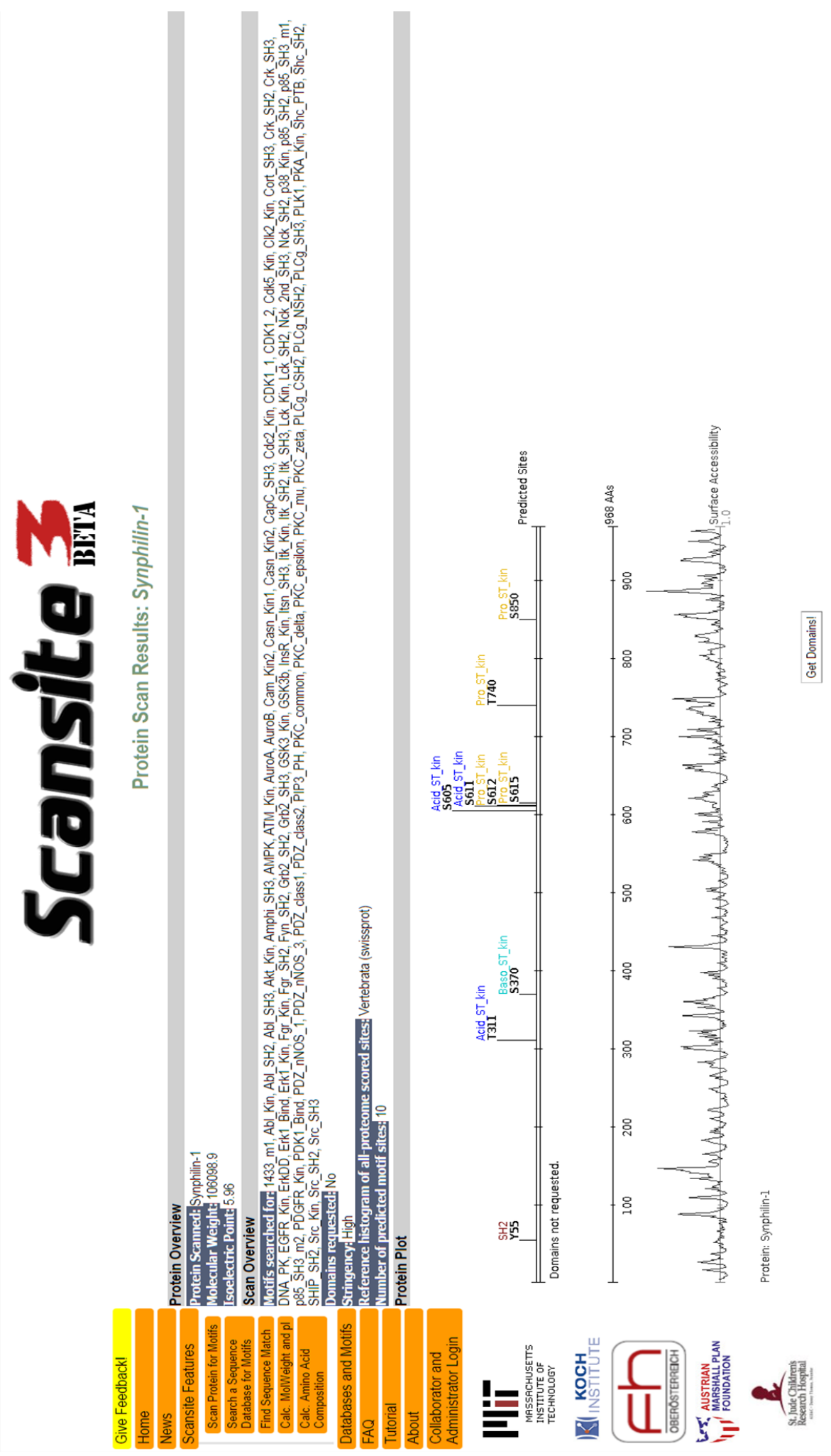


Appendix 1: Scansite 3.0 in-silico analysis page 2

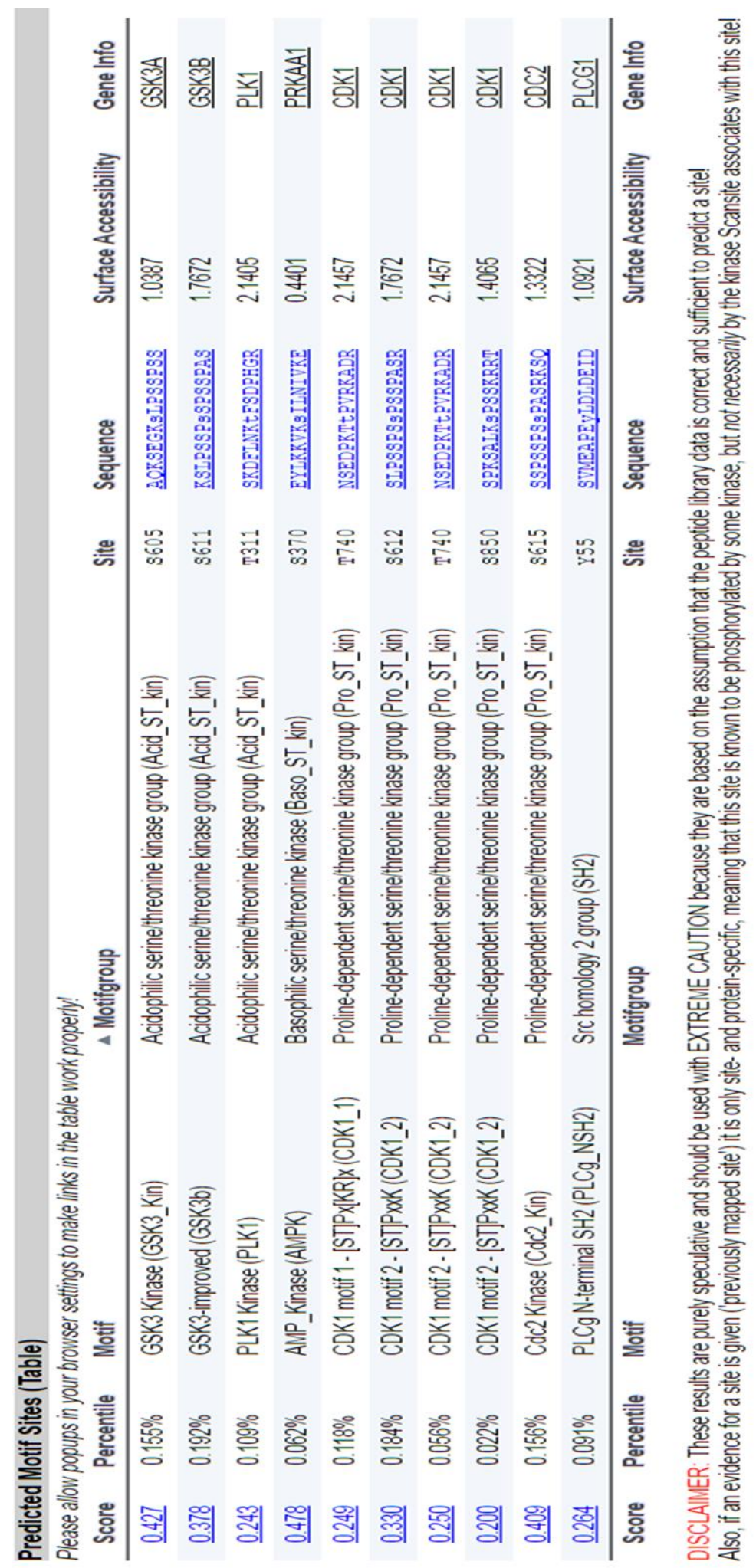

